Bubbles and drops in turbulent Taylor-Couette flow: numerical modelling and simulations 


\section{Bubbles and drops in turbulent Taylor-Couette flow: numerical modelling and simulations}

Vamsi Spandan Arza 
Graduation committee

Prof. Dr. ir. J.W.M. Hilgenkamp (chairman) University of Twente

Prof. Dr. D. Lohse (supervisor)

University of Twente

Prof. Dr. R. Verzicco (co-supervisor)

University of Twente

Prof. Dr. ir. J. J. W. van der Vegt

University of Twente

Prof. Dr. J. G. M. Kuerten

University of Twente

Prof. Dr. B. J. Boersma

Delft University of Technology

Prof. Dr. S. Sarkar

University of California, San Diego

Cover design: Vamsi Spandan Arza. Volume plot of dissipation in a Taylor-Couette flow system injected with finite-size bubbles.

Copyright (C) 2017 by Vamsi Spandan Arza, Enschede, The Netherlands. All rights reserved. No part of this book may be reproduced, stored in a retrieval system, or transmitted in any form or by any means, without the written permission of the author.

Printed by GildePrint

DOI: $10.3990 / 1.9789036543682$

ISBN: 978-90-365-4368-2

URL: https://dx.doi.org/10.3990/1.9789036543682 


\title{
Bubbles and drops in turbulent Taylor-Couette flow: numerical modelling and simulations
}

\author{
DISSERTATION
}

\author{
to obtain \\ the degree of doctor at the University of Twente, \\ on the authority of the rector magnificus, \\ Prof. Dr. T.T.M. Palstra, \\ on account of the decision of the graduation committee, \\ to be publicly defended, \\ on Friday the 14th of July 2017 at 16:45
}

by

Vamsi Spandan Arza

Born on $7^{\text {th }}$ March 1991

in Eluru, Andhra Pradesh, India 
This dissertation has been approved by the promotors:

Prof. Dr. Detlef Lohse and Prof. Dr. Roberto Verzicco 


\section{Contents}

1 Introduction 1

1.1 Turbulence and multiphase flows . . . . . . . . . . . . . . 2

1.2 Challenges in numerical simulations . . . . . . . . . . . . . . 5

1.3 Multiphase Taylor-Couette turbulence . . . . . . . . . . . . . . 6

1.4 Governing equations and numerical details . . . . . . . . . . . . . . . 10

1.5 Outline of the thesis . . . . . . . . . . . . . 11

2 Drag reduction with sub-Kolmogorov spherical bubbles 13

2.1 Introduction . . . . . . . . . . . . . . . . . . . . 14

2.2 Governing Equations and Numerical Details . . . . . . . . . . . . . 17

2.2.1 Carrier phase . . . . . . . . . . . . . 17

2.2 .2 Dispersed phase . . . . . . . . . . . . . . . . . 18

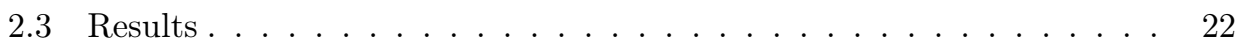

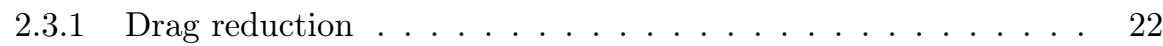

2.3.2 Carrier phase velocity fields . . . . . . . . . . . . . . . . 25

2.3.3 Motion of dispersed phase . . . . . . . . . . . . . . . . . . 30

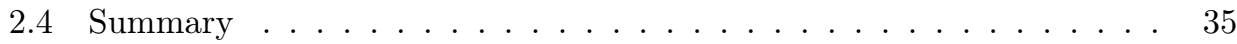

3 Deformation and orientation statistics of sub-Kolmogorov drops $\quad 37$

3.1 Introduction . . . . . . . . . . . . . . . . . . 38

3.2 Governing Equations and Numerical Details . . . . . . . . . . . . . . . 41

3.3 Results. . . . . . . . . . . . . . . . . . . . 45

3.3.1 Droplet shape distribution . . . . . . . . . . . . . . 45

3.3.2 Orientational behaviour of the drops . . . . . . . . . . . 51

3.3.3 Effect of viscosity ratio . . . . . . . . . . . . . . 57

3.4 Summary .......................... 60

4 Deformable bubbles with enhanced Euler-Lagrange tracking $\quad 63$ 
4.1 Introduction . . . . . . . . . . . . . . . . . . . 64

4.2 Governing Equations . . . . . . . . . . . . . . . . 66

4.2.1 Dynamics of carrier phase and shape tensor . . . . . . . . 66

4.2 .2 Modelling of effective forces . . . . . . . . . . . . . . 67

4.2 .3 Simulation parameters . . . . . . . . . . . . . . . . . . 69

4.3 Results. . . . . . . . . . . . . . . . . . . . 69

4.3.1 Global transport quantities . . . . . . . . . . . . . . 69

4.3.2 Mean bubble concentration profiles . . . . . . . . . . . . 71

4.3.3 Bubble shape and orientation . . . . . . . . . . . . . . . 73

4.4 Summary . . . . . . . . . . . . . . . . . . 76

5 Immersed boundary method coupled with interaction potential for $\begin{array}{ll}\text { deformable interfaces and membranes } & \mathbf{7 9}\end{array}$

5.1 Introduction . . . . . . . . . . . . . . . . 80

5.2 Governing equations and numerical scheme $\ldots \ldots \ldots \ldots$

5.2.1 Interaction potential approach for deformation . . . . . 88

5.3 Liquid-liquid interface dynamics using the potential approach . . . . . 91

5.3.1 Deformation of a neutrally buoyant drop in shear flow . . . . 92

5.3.2 Dynamics of a liquid-liquid interface deforming in cross-flow . . 96

5.4 Data structures and pseudo code for Lagrangian mesh parallelisation . 99

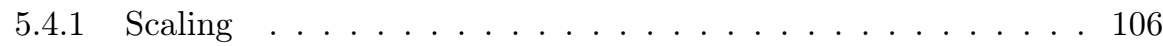

$5.5 \quad$ Summary . . . . . . . . . . . . . . . . . . . . . . 108

6 Coarse grained algorithms for the immersed boundary method 109

6.1 Introduction . . . . . . . . . . . . . . . . . . . . 110

6.2 Fast moving least squares for the immersed boundary method . . . . . 113

6.3 Decoupling the Lagrangian meshes . . . . . . . . . . . . . . . . 119

6.4 Coarse-grained collision detection . . . . . . . . . . . . . . . 123

6.5 Summary . . . . . . . . . . . . . . . . . . . 126

7 Finite-size bubbles and drops in Taylor-Couette flow 129

7.1 Introduction . . . . . . . . . . . . . . . . . . 130

7.2 Numerical details . . . . . . . . . . . . . . . . . . . . . . . 131

7.3 Results . . . . . . . . . . . . . . . . . . . . . . . 133

7.4 Summary . . . . . . . . . . . . . . . . . . 139

$\begin{array}{lll}8 & \text { Conclusions } & 141\end{array}$

$\begin{array}{ll}\text { Summary } & 159\end{array}$ 
CONTENTS

$\begin{array}{ll}\text { Samenvatting } & 162\end{array}$

$\begin{array}{ll}\text { Scientific Output } & 167\end{array}$

$\begin{array}{ll}\text { Acknowledgments } & 170\end{array}$ 
CONTENTS 
Chapter 1

\section{Introduction}




\subsection{Turbulence and multiphase flows}

Life, as we know, would not exist without fluids and the behaviour they exhibit. Without the intention of being exhaustive, here are a few examples. The motion of blood in the circulatory system of animals is crucial for the transport of oxygen and nutrients to various organs. Similar to animals, plants have their own circulatory system to transport water and other nutrients they absorb from soil. Approximately $70 \%$ of Earth's surface is covered by oceans which provide home to some of the most diverse living forms. In addition to playing a vital role in the origin and sustenance of life, fluids are also an important part of everyday human activity. A variety of cooking processes such as boiling, frying, baking and pickling rely on fluid dynamics. Transport of millions of people and objects everyday through air, road and water is only possible due to fundamental fluid dynamics phenomena such as propulsion, lift, combustion etc. The paper used to print this thesis was manufactured after careful processing of pulp, while the text itself is printed through high precision impact of pico-litre $\left(10^{-9} \mathrm{ml}\right)$ ink droplets. The examples are innumerable and it is thus no wonder that even after centuries of inquiry into understanding the behaviour of fluids, new and interesting phenomena still tickle the curiosity of scientists [1].

One such phenomenon that has demanded specific attention from the fluid dynamics community for a long time is turbulence [2]. The famous physicist, Richard Feynman described turbulence as "the most important unsolved problem in classical physics". In simple words, turbulence is a regime of fluid dynamics characterised by chaotic and irregular motion. While it is relatively straightforward to describe turbulence qualitatively, understanding and quantifying it from a mathematical point of view is far from trivial. The first known quantitative description on the occurrence of turbulence in fluid flow was provided by Osborne Reynolds in a ground breaking experiment in 1883 [3] where he studied the behaviour of water flow in a transparent pipe injected with a small jet of dye ${ }^{1}$. Through his experiments (see figure 1.1 (a)), he demonstrated that the general nature of fluid motion depends on the relation between physical constants of the fluid (density and viscosity), the velocity of the fluid and a relevant physical length scale in the flow. Following this discovery, a variety of theoretical and experimental studies focussed on understanding the nature of turbulent flows. The advent of computers brought to light a new paradigm of line of scientific inquiry and over the last few decades numerical computing has proven to be an extremely efficient and faithful tool in understanding turbulence.

The major bottleneck in simplifying turbulent flows is the existence of a wide

\footnotetext{
${ }^{1}$ The word turbulence was coined only a few years later in 1887 by Lord Kelvin.
} 
range of length and time scales in the flow as can be seen in some typical examples of turbulent flows shown in figure 1.1(b),(c),(d),(e). Attempts at modelling turbulence are backlashed by the fact that the number of unknowns are more than the number of governing equations available (typically known as the 'closure problem' of turbulence).

With the combined effort of experiments, theory and numerical simulations, considerable progress has been made in understanding turbulence in a system with a single fluid phase. However, most turbulent flows occurring in nature and industrial applications comprise of more than one phase. For example, dust particles and ash being dispersed into the atmosphere by a volcanic eruption (figure 1.1(b)); flow across the hull of a submarine which comprises of a turbulent mixture of water and entrapped air bubbles (figure 1.1(c)); clouds, which are an agglomeration of several minuscule water droplets immersed in a turbulent atmospheric environment; the combined flow of gas and liquid in long pipelines or chemical reactors, plankton dispersion in sea, fuel sprays etc. The bubbles, drops and particles dispersed in all these flows have their own unique identity in how they respond to and in turn affect the underlying flow features. This brings into play new length and time scales in the flow, in addition to the scale separation inherent to turbulence itself and this makes turbulent multiphase flows an exciting yet challenging field of study.

Theoretical descriptions of multiphase flows are far from providing a unifying view of the various physical mechanisms that are active. Experimentally, it is possible to measure global properties in an accurate and reliable manner. However, in order to provide a satisfying description of the underlying physics, measurements of local quantities become a necessity. It is not possible to measure all local quantities in experiments as they either become too invasive or are not accurate enough to be reliable. For example, consider a turbulent mixture of bubbles and liquid flowing in a channel. In experiments, while one can easily quantify the power required to pump the mixture or measure the fluctuations in velocity at a fixed point, it is extremely challenging and close to impossible to gather information on the shape of individual bubbles at different time instants or the kind of forces acting on the them. The third alternative for studying multiphase flows is provided by numerical computing which gives access to the complete flow field and desired information on the dispersed bubbles, drops or particles. However, numerical tools can become very restrictive in simulating large scale systems relevant for fundamental research or industrial applications [5]. 
(a)

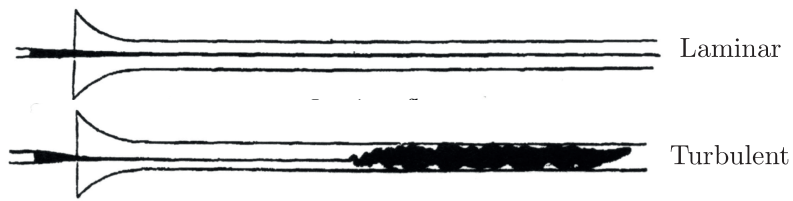

\section{(b)}

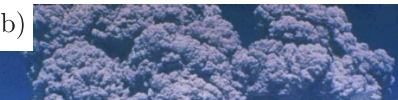

(c)

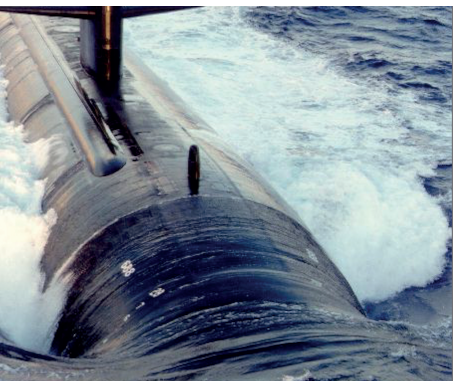

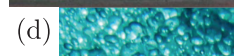
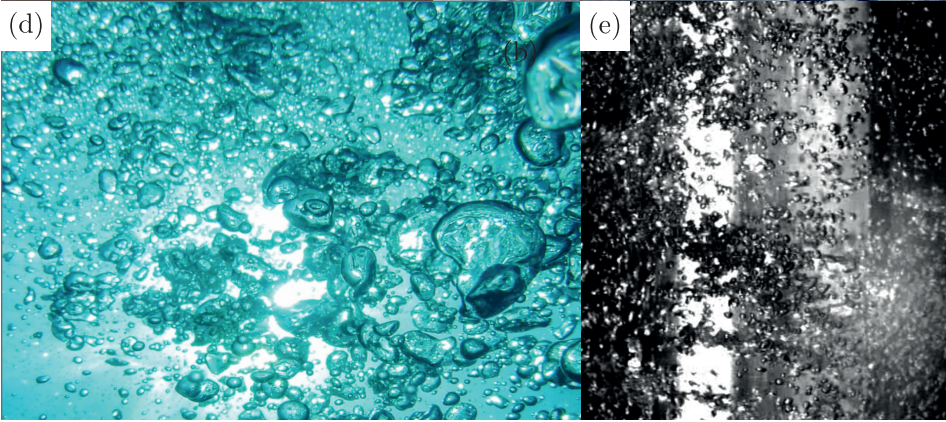

Figure 1.1: (a) Flow of water observed in a transparent pipe as depicted by Osborne Reynolds in his paper [3]. Water flows from left to right and dye (shown in black) flows in the centre of the pipe. Beyond a critical velocity of the flow, onset of turbulence can be observed through chaotic mixing of the dye. (b) Turbulent mixture of ash and dust particles from a volcanic eruption (c) Laminar and turbulent flow over the hull of a submarine (d) Bubbles of different sizes and shapes in water (e) Bubbles in a turbulent flow from the experiments of [4]. (Source for images (b),(c),(d): Wikipedia). 


\subsection{Challenges in numerical simulations}

A desirable trait in any numerical tool is that it fully resolves all the length and time scales present in the flow. At the continuum level, this is achieved through Direct Numerical Simulations (DNS) and if done correctly it yields the 'exact' solution of the governing equations [6]. DNS has been the de-facto standard to gain insights into single phase turbulent flows for the past several decades and is considered the most reliable and trusted numerical tool even today. Given the high levels of precision, DNS allows us to study flow systems with a great amount of detail and it is primarily used to gain a deeper fundamental understanding of the problem under consideration. The major bottleneck in using DNS without restriction is the massive increase in the computing power required with increasing separation of scales in the flow. This problem is partially overcome with the use of supercomputers which allow simultaneous computations of a flow system on thousands of computing processors.

In comparison to single phase flows, DNS of multiphase flows is extremely challenging and expectedly lagging behind [7]. For example, computations of billions of fully resolved bubbles, drops or particles in a highly turbulent flow is unimaginable not only due to the immense computing power required but also due to shortcomings in the mathematical and algorithmic framework required to treat the sharp gradients in the density, viscosity, pressure and velocity across interfaces in a computationally inexpensive and efficient manner. Nonetheless, given the significant progress made in this field over the last two decades, it is now becoming clear that instead of developing a general DNS framework for studying all types of multiphase flows, each flow system has to be tackled on its own merit.

A point-particle Euler-Lagrangian approach is the preferred choice for flows laden with particles, bubbles or drops with a fixed shape and a size smaller than the smallest length scale in the carrier flow (typically the Kolmogorov scale $\eta_{K}$ ). In this approach, the dynamics of the carrier fluid can be fully resolved through DNS, while it is practically impossible to do the same for the dispersed phase due to limitations in resolutions. Thus, the exchange of momentum, mass or energy between the dispersed phase and the carrier fluid is modelled through empirical correlations. Within the point-particle approach, the dispersed phase can be advected with or without considering its back-reaction force onto the carrier phase; otherwise called the two-way coupled or one-way coupled approach, respectively. The consequences of this choice are discussed in the next section.

When the typical size of dispersed phase is larger than the smallest length scale in the flow, fully resolved simulations for both phases becomes unavoidable. Techniques 
such as the immersed boundary method (IBM), Physalis, front-tracking, VoF, level-set are at the forefront in tackling such problems [5]. VoF and level-set use a single set of governing equations for the whole flow field ('one-fluid formulation') and the different phases in the flow are identified using a marker function that is advected based on the local flow velocity. These methods excel at flows involving large deformations of fluid-fluid interfaces; for example, high speed impact of droplets on substrates, fragmentation of liquid jets, disintegration and breakup of drops or bubbles etc. Front-tracking makes use of a secondary unstructured mesh which keeps track of the boundary between immiscible fluids present in the system and has been used primarily to study the interaction of bubbles and drops within a weakly turbulent carrier flow. The immersed boundary method (IBM) which was first developed to study flow around heart valves has only recently been used in simulations of multiphase flows. IBM has been instrumental in simulations of highly turbulent flows laden with rigid spherical or ellipsoidal particles. Extensions to include bubbles and drops within the IBM framework is non-trivial and only a limited number of studies exist in literature.

From the above discussion, it is clear that the choice of the numerical tool to simulate a multiphase flow strongly depends on the type of flow being studied. In flow regimes, where current state of the art techniques fall short, new models and algorithms need to be developed which will further progress the field of computational multiphase flows.

\subsection{Multiphase Taylor-Couette turbulence}

In this thesis, we focus on understanding the behaviour of bubbles and drops in a canonical flow set up, namely Taylor-Couette (TC) flow using various numerical tools.

In single-phase TC flow, a fluid is confined between and driven by two independently rotating co-axial cylinders while in a multiphase system, bubbles and drops of various sizes and physical properties are injected into the confined fluid. A schematic of a multiphase TC system is shown in figure 1.2 where bubbles and drops of different sizes $\left(d_{p}\right)$ and physical properties (density, viscosity, surface tension etc.) are dispersed into the flow. TC flow has been one of the classical models to study and understand various concepts in fluid dynamics for the past several decades due to various reasons: (i) it is geometrically simple, (ii) it is a closed flow system with exact global balances between the driving and dissipation, and (iii) it is mathematically well defined through the Navier-Stokes equations and the respective boundary conditions. A major motivation for studying multiphase TC turbulence is to understand the effect 
of the dispersed bubbles or drops on the overall friction or drag experienced by the rotating cylinders.

Frictional losses in the form of drag in turbulent flows are a major drain of energy in applications related to process technology, naval transportation, and transport of liquefied natural gas in pipelines [8]. It has been known for a long time that the injection of a small concentration of a dispersed phase into the carrier fluid can result in significant drag reduction which makes it of interest for both fundamental scientific research and industrial applications. Drag reduction through injection of a secondary phase has been demonstrated in many physical systems in the past, like bubbles injected into a turbulent boundary layer over a flat plate [9-11], in channel flows [12-17], and Taylor-Couette (TC) flow [4, 18-22]; the reader is referred to Ceccio et al. [8] and Murai et al. [23] for more detailed reviews. The magnitude of reduction in drag or the driving force for a two-phase system when compared to a single phase system can be massive; van Gils et al. [4] showed drag reduction of up to $40 \%$ with just $4 \%$ of bubbles dispersed into TC flow.

While there have been a number of experimental studies demonstrating the effect of bubbles on the drag in a two-phase TC flow, numerical studies have been fairly limited. As discussed in the previous section, the computational burden of fully resolving the interactions between thousands of bubbles and the carrier phase is massive, thus making it extremely challenging to reach high levels of turbulence. In previous studies, this problem has been dealt with by advecting point like bubbles in a turbulent flow field in a one-way coupled Euler-Lagrangian manner $[24,25]$. Since one-way coupled simulations involve only advecting the dispersed phase without any back-reaction onto the carrier phase, these simulations prevent investigation of any form of drag modification mechanisms that maybe active in the flow. This problem is overcome by two-way coupled simulations [26], namely by implementing the momentum exchange between the bubbles and the carrier phase. While extremely useful, point-particle simulations still only allow bubbles or drops which are smaller than the Kolmogorov scale (sub-Kolmogorov) and techniques such as VOF, front-tracking or IBM become necessary to study bubbles or drops larger than the Kolmogorov scale (finite-size). Until now, there have been no numerical counterparts for the experimental studies of van Gils et al. [4] which would require simulations of finite-size bubbles or drops in a highly turbulent TC flow.

Various theories have been suggested to explain the origin of the drag reduction effect that has been observed in multiphase systems; among them are theories based on effective compressibility [27, 28], disruption of coherent vortical structures present in the single phase flow [26], and also effects of bubble deformability [4, 21, 22]. However, 
the exact mechanism is still not clear and it can be expected that different mechanisms such as those mentioned above are dominant in different flow regimes. As discussed previously, the simplicity of the TC system allows for high precision numerical simulations and experimental measurements which makes it an ideal playground to study and understand the phenomenon of drag reduction under the influence of a dispersed phase like bubbles or drops.

From the schematic shown in figure 1.2 and the previous discussion it is apparent that bubbles and drops of varying sizes and physical properties can be present in a multiphase TC system. The first question we ask in this thesis is: What are the parameters relevant for attaining drag reduction when sub-Kolmogorov spherical bubbles are injected into a turbulent TC flow. Experimental studies in the past measure a consistent decrease in the drag reduction with increasing Reynolds number upon injection of sub-Kolmogorov spherical bubbles into the flow. However, the limitation in experiments is that the governing parameters are inherently coupled and numerical simulations allow us to independently control these parameters and isolate their individual effects on drag reduction in a systematic manner.

When the stretching forces acting on the sub-Kolmogorov bubbles and drops become dominant over the surface tension forces, the isotropy in the shape of the dispersed phase is lost. In such a situation, we ask how would the deformation and orientation statistics of the dispersed phase depend on the local flow conditions and in turn affect them. Does the extent of deformability of sub-Kolmogorov bubbles affect drag reduction in any manner and if so, how is it related to the shape and orientation of the dispersed phase?

The next question we focus on is, what are the consequences when the dispersed bubbles or drops are larger than the Kolmogorov length scale (finite-size) in the flow. Experiments have shown that a mere $4 \%$ injection of bubbles into a highly turbulent TC flow can reduce drag on the driving cylinders up to $40 \%$ [4]. What is the exact physical mechanism behind this massive drag reduction and how can we understand it from a quantitative manner? Simulating finite-size bubbles in highly turbulent flows would require complex algorithms and massive computational power. Is it possible to simulate such systems with the algorithms currently at hand or is there a need for novel methods which can scale up numerical simulations of multiphase flows?

We will answer these questions through the use of numerical simulations and study different flow regimes with bubbles and drops of different sizes and properties which will help us take a step forward in understanding the underlying physics behind two-phase TC drag reduction. 


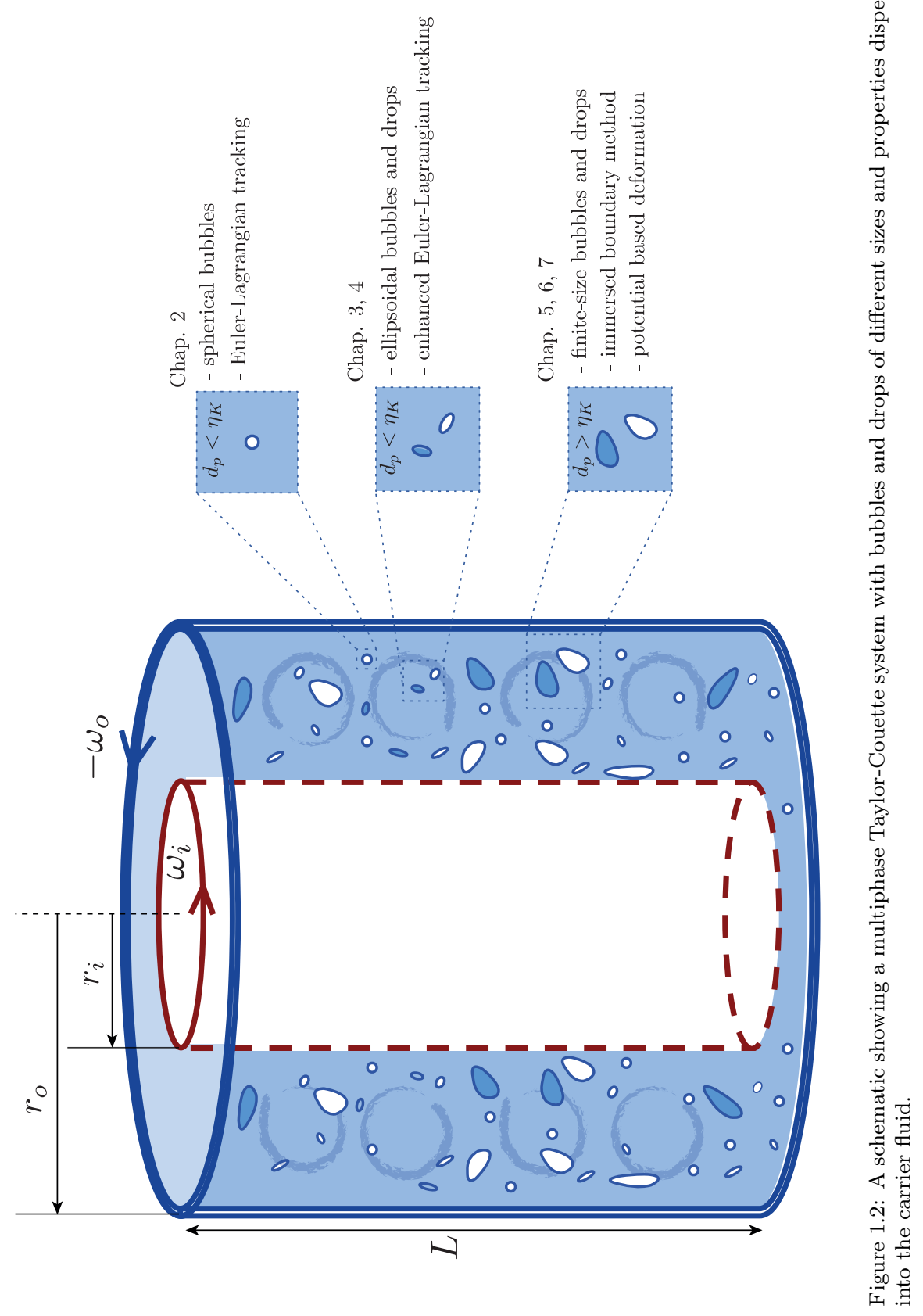




\subsection{Governing equations and numerical details}

In this thesis, the dynamics of the carrier fluid is solved using DNS of the Navier-Stokes equations which read as follows:

$$
\begin{gathered}
\frac{\partial \mathbf{u}}{\partial t}+\mathbf{u} \cdot \nabla \mathbf{u}=-\nabla p+\frac{1}{R e} \nabla^{2} \mathbf{u}+\mathbf{f}_{b}, \\
\nabla \cdot \mathbf{u}=0 .
\end{gathered}
$$

$R e$ is the Reynolds number of the flow and is a measure of the relative strength of the inertial forces to the viscous forces acting on the fluid elements. It is defined based on a characteristic length scale $L$ and velocity scale $U$, i.e. $R e=U L / \nu ; \nu$ is the kinematic viscosity of the fluid. $\mathbf{u}, p$ are the velocities and pressure in the flow while $\mathbf{f}_{\mathbf{b}}$ is a volumetric body force acting on the fluid. In this thesis, we keep the outer cylinder stationary and rotate only the inner cylinder with an angular velocity of $\omega_{i}$. The Reynolds number in such a system is then written as $R e_{i}=\left(r_{o}-r_{i}\right) U_{i} / \nu$, where $r_{i}$ and $r_{o}$ are the inner and outer cylinder radii, respectively and $U_{i}=r_{i} \omega_{i}$ is the velocity of the inner cylinder.

An energy conserving second-order centred finite-difference scheme with velocities on a staggered grid is used for spatial discretisation of the governing equations; explicit Adams-Bashforth scheme is used to discretise the non-linear terms while an implicit Crank-Nicholson scheme is used for the viscous terms. Treating all the viscous terms implicitly results in a large sparse matrix whose inversion is avoided by an approximate factorisation and the sparse matrix is then transformed into three tridiagonal matrices (one for each direction) and solved using Thomas' algorithm. Time integration is performed via a self starting fractional step third-order Runge-Kutta (RK3) scheme. The pressure required to enforce mass conservation is computed by solving a Poisson equation for a pressure correction. Periodic conditions are employed in the azimuthal and axial directions and the consequences of this choice are discussed in subsequent chapters. These schemes have already been tested extensively previously in the context of single phase flows for a variety of flow configurations; additional details of the numerical scheme can be found in previous works [29-31].

As mentioned previously, for the dispersed phase we use a combination of techniques to study bubbles and drops of different sizes with different physical properties as shown in figure 1.2. While the governing equations and numerical details for the dispersed phase are discussed in the following chapters, here we give a brief overview of the techniques used. Bubbles which are smaller than the Kolmogorov scale (sub-Kolmogorov) and are rigid in shape due to strong surface tension forces are 
simulated using a two-way coupled point particle Euler-Lagrange approach. When the relative strength of the viscous stretching forces become stronger than the surface tension forces, sub-Kolmogorov deformable bubbles and drops are tracked by coupling tradition Euler-Lagrangian tracking with a sub-grid deformation model. Finally, to simulate finite-size (larger than Kolmogorov scale) bubbles and drops which can deform with several degrees of freedom, we use IBM coupled with an interaction potential approach for deformable interfaces.

\subsection{Outline of the thesis}

This thesis is organised as follows. In $\S 2$, we study the influence of the effective buoyancy of sub-Kolmogorov bubbles on the net drag reduction in TC flow. In $§ 3$, we use a sub-grid deformation model to study the deformation and orientation statistics of sub-Kolmogorov neutrally buoyant drops and in $\S 4$, we couple the deformation model with two-way coupled Euler-Lagrangian tracking to study the influence of deformable sub-Kolmogorov bubbles on the drag of the rotating cylinders. In $\S 5$, we show and discuss how the deformation dynamics of closed liquid-liquid interfaces (for example drops and bubbles) can be replicated with use of a phenomenological interaction potential model. The interaction potential model is coupled with the immersed boundary method which allows us to simulate finite-size deformable bubbles and drops in turbulent flows. In $\S 6$, we discuss multiple optimisation and coarse-grained algorithms which are useful in scaling up the coupled interaction potential-immersed boundary method for large scale multiphase flow simulations. We use the methods described in $\S 5$ and $\S 6$ to simulate finite size bubbles and drops in turbulent TC flow in $\S 7$ and to understand the mechanism of drag reduction in a completely different regime as compared to $\S 2$ and $\S 4$. We conclude the thesis in $\S 8$ with a summary and an outlook on future work. 


\section{Chapter 2}

\section{Drag reduction with}

\section{sub-Kolmogorov spherical}

\section{bubbles}

Based on: Vamsi Spandan, Rodolfo Ostilla-Monico, Roberto Verzicco, Detlef Lohse, 'Drag reduction in numerical two-phase Taylor-Couette turbulence using an Euler-Lagrange approach' Journal of Fluid Mechanics, Vol. 798, 411-435, 2016 (arXiv id: 1510.01107). 


\section{$2.1 \quad$ Introduction}

In this chapter we study the influence of sub-Kolmogorov spherical bubbles on the overall drag on the rotating cylinder in a two-phase Taylor-Couette flow. We focus on understanding the effects of the dispersed phase on the dynamics of the carrier phase through independent control of the governing parameters and its subsequent consequences on the torque required to drive the system.

As discussed in $\S 1$, TC flow is one of the paradigmatic systems to study turbulence in wall-bounded systems (see [32] for a recent review). The geometry of the TC system can be described using the radius ratio $\eta=r_{i} / r_{o}$ and the aspect ratio $\Gamma=L / d$, where $r_{i}, r_{o}$ are the radius of the inner and outer cylinders respectively, $d=r_{o}-r_{i}$ is the gap width and $L$ is the height of the cylinders (cf. figure 2 in $\S 1$ ). Dimensionless radial and axial distances are defined in the form of $\tilde{r}=\left(r-r_{i}\right) / d$ and $\tilde{z}=z / d$, respectively.

For a single phase TC system, at very low driving the flow is purely azimuthal and in a laminar state. Once the driving of the flow is stronger than a critical value, the purely azimuthal, stable laminar flow is disrupted, leading to the formation of large scale Taylor rolls. Increasing the driving further leads to the onset of interesting flow regimes such as Taylor vortex flow, wavy vortex flow, modulated wavy vortex flow, turbulent Taylor vortices etc. The various regimes that can be observed in a single phase turbulent TC flow have been summarised by Andereck et al. [33] for low Reynolds numbers and recently extended for the highly turbulent case by Ostilla-Monico et al. [34].

The global response of the TC system to the driving can be quantified in terms of the friction factor

$$
C_{f}=\frac{\tau_{w}}{\frac{1}{2} \rho U_{i}^{2}},
$$

where $\tau_{w}$ and $U_{i}$ are the averaged wall shear stress and velocity of the inner cylinder, respectively. The friction factor can be seen as the dimensionless drag of the system on the inner cylinder. For pipe flow, the corresponding friction factor is the most common way to express the wall drag in dimensionless form [35]. For Taylor-Couette flow, alternatively, the response of the system can be quantified in terms of a generalised Nusselt number $N u_{\omega}$, which is the angular velocity transport from the inner to the outer cylinder, non-dimensionalised by its value for laminar flow $J_{\text {lam }}[36]$, i.e.,

$$
N u_{\omega}=\frac{J}{J_{\text {lam }}}, \quad \text { with } \quad J=r^{3}\left(\left\langle u_{r} \omega\right\rangle_{A, t}-\nu \partial_{r}\langle\omega\rangle_{A, t}\right),
$$

where $\langle\ldots\rangle_{A, t}$ represents averaging in the two homogeneous (azimuthal and axial) directions and also in time and $J_{\text {lam }}=2 \nu r_{i}^{2} r_{o}^{2}\left(\omega_{1}-\omega_{2}\right) /\left(r_{o}^{2}-r_{i}^{2}\right)$. The relation 
between the Nusselt number and the friction factor reads

$$
C_{f}=\frac{2 \pi L N u_{\omega} J_{\mathrm{lam}}}{\frac{1}{2} r_{i} U_{i}^{2}} .
$$

In a two-phase TC system a secondary phase is dispersed into the carrier phase (here bubbles dispersed into water) as shown in figure 2 of $\S 1$. The dispersed phase is transported by virtue of various forces acting on them such as buoyancy $\left(F_{B}\right)$, drag $\left(F_{D}\right)$, lift $\left(F_{L}\right)$, added mass $\left(F_{A}\right)$, history forces $\left(F_{H}\right)$ etc. The dispersed phase in turn has a back-reaction force on the carrier flow, thus affecting the angular velocity transport between the two cylinders. This may lead to a change in the torque required to drive the system. A net percentage drag reduction $(D R)$ for a two-phase TC system can be quantified according to equation (2.4).

$$
D R=\frac{\left\langle N u_{\omega}\right\rangle_{s}-\left\langle N u_{\omega}\right\rangle_{t}}{\left\langle N u_{\omega}\right\rangle_{s}} \times 100=\frac{\left\langle C_{f}\right\rangle_{s}-\left\langle C_{f}\right\rangle_{t}}{\left\langle C_{f}\right\rangle_{s}} \times 100
$$

$\langle\ldots\rangle_{s}$ and $\langle\ldots\rangle_{t}$ correspond to single phase and two-phase systems, respectively.

The control parameters for the dispersed phase in the case of sub-Kolmogorov bubbles dispersed into TC flow are the bubble diameter $\left(d_{b}\right)$, density ratio (for bubbles dispersed into water $\left.\rho_{p} / \rho_{f} \ll 1\right)$, global volume fraction $\left(\alpha_{g}=N_{p} V_{p} / V\right.$, where $N_{p}$ is total number of dispersed particles, $V_{p}$ is the volume of individual particle and $V=\pi\left(r_{o}{ }^{2}-r_{i}{ }^{2}\right) L$ is the total volume of the TC system) and Froude number $F r=$ $\omega_{i} \sqrt{r_{i} / g}$ which is the square root of the ratio of centripetal and the gravitational accelerations. Since $\mathrm{Fr}$ is a ratio of forces in two different directions, it does not describe the equilibrium position of the bubbles in the TC gap width. However, it can be interpreted as the relative strength of the buoyancy of bubbles as compared to the driving in the TC system, as has been done in previous studies [18, 19, 37, 38].

Shiomi et al. [39] performed one of the earliest experiments on two-phase TC flow by studying the various flow patterns that develop in a two-phase mixture confined in a concentric annulus. They observed various patterns such as dispersed bubbly, single spiral, double spiral, and triple spiral flows. Djeridi et al. [40] studied the bubble capture and migration patterns in a TC cell for two different configurations, namely (i) with a free upper surface and (ii) with a top stationary wall. In a subsequent study Djeridi et al. [41] found different spatial structures while using air bubbles and cavitation bubbles separately as the dispersed phase. The focus of these studies was primarily on understanding the different flow patterns that developed in a two-phase TC system, but not on the modification of the torque required to drive the cylinder. Murai et al. $[18,19]$ demonstrated experimentally that a tiny percentage of the 
dispersed phase ( $0.1 \%$ volume fraction of bubbles dispersed in silicone oil) can reduce the driving torque on the inner cylinder up to $25 \%$. The maximum inner cylinder Reynolds number reached in these experiments was $R e_{i}=4500$ and they found that the overall drag reduction decreased with increasing Reynolds number with almost negligible drag reduction at $R e_{i}=4000$. With the help of particle tracking velocimetry (PTV) Yoshida et al. [37] studied the relationship between the observed drag reduction and changes in the vortical structures of the bubbly TC system. More recently Watamura et al. [38] investigated the effect of micro-bubbles on the properties of azimuthal waves found in TC flow $\left(\left\langle R e_{i}\right\rangle_{\max }=1000\right)$.

In the highly turbulent regime $\left(R e_{i}=10^{5}-10^{6}\right)$, drag reduction of up to $25 \%$ was achieved by injecting millimetric sized bubbles into the TC flow by van den Berg et al. $[20,21]$. In this regime, it has been demonstrated that deformability of the bubbles can be a deciding factor in achieving strong drag reduction of up to 40\% [4]. The torque required to drive the inner cylinder in a two-phase TC system (or a bubbly-TC system) depends on various control parameters as mentioned previously. However, in experiments it is not possible to control all these parameters independently, thus making it challenging to study the individual effect of each control parameter on the system. For example as shown in experiments by Murai et al. [18, 19], the Froude number $\mathrm{Fr}$, (which is directly related to the velocity of the inner cylinder) cannot be controlled independently from the inner cylinder Reynolds number $\left(R e_{i}\right)$. Additionally the diameter of the bubbles dispersed into the gap width depends on the local shear in the flow at the point of injection of the bubbles. In contrast to experiments, numerical simulations allow for more flexible control over the parameters and to explore the underlying physics in detail.

Overall, numerical studies on two-phase Taylor-Couette flows have been fairly limited. One-way coupling simulations have provided answers to how bubbles injected into TC flow organise themselves within the cylinder gap [24, 25]. However, as discussed previously in $\S 1$, one-way coupling simulations involve only advecting the dispersed phase based on the local flow conditions without any back-reaction onto the carrier phase which prevents investigation of drag modification with bubbles. Two-way coupling simulations are crucial in order to investigate the effect of bubbles on the dynamics of a carrier phase. In the context of homogeneous isotropic turbulence it has already been shown through two-way coupled simulations, that at large scales the point-like bubbles reduce the intensity in the turbulence spectrum as compared to one-way coupling simulations [42]. Using a two-way coupled Euler-Lagrange scheme, Sugiyama et al. [26] reproduced the drag reduction observed in experiments by Murai et al. [18] and concluded that spherical microbubbles influence the TC system 
by disrupting the coherent vortical structures and found that the drag reduction decreased at higher $R e_{i}$; in those simulations the maximum $R e_{i}$ reached was 2500 where they observed a drag reduction of about $5 \%$.

In this chapter we use a similar approach by simulating the carrier phase using DNS and tracking the dispersed phase in a Lagrangian manner along with two-way coupling between the phases to extend the $R e_{i}$ limit to 8000 and gain more insight into two-phase TC drag reduction. We want to find out the effect of bubbles on the driving torque at these higher $R e_{i}$. The experiments by Murai et al. $[18,19]$ found almost negligible torque modification at $R e_{i}=4000$, but without independent control over the Froude number $\mathrm{Fr}$ due to its implicit dependence on the angular frequency of the inner cylinder and consequently on the Reynolds number $R e_{i}$. Will the drag reduction be sustained if $\mathrm{Fr}$ of the bubbles is controlled or would there be drag enhancement? How does the trajectory of a bubble depend on the level of turbulence in the system and the Fr number? These are the questions we attempt to answer in this chapter.

\subsection{Governing Equations and Numerical Details}

\subsubsection{Carrier phase}

The dynamics of the carrier phase is computed by solving the Navier-Stokes equations in cylindrical coordinates; refer to equations 1.1 and 1.2 in $\S 1$. In point-particle Euler-Lagrange type approaches, $\mathbf{f}_{b}$ is the back-reaction force from the dispersed phase onto the carrier phase; this term is ignored in a one-way coupling simulation. In a two-way coupling simulation $\mathbf{f}_{b}$ is calculated according to $[5,26,42,43]$.

$$
\mathbf{f}_{b}=\sum_{i=0}^{N_{p}}\left(\frac{D \mathbf{u}}{D t}-\mathbf{g}\right) V_{p} \delta\left(\mathbf{x}-\mathbf{x}_{p}(t)\right) .
$$

The back-reaction force $\mathbf{f}_{b}$ is calculated at the exact position of each particle $\mathbf{x}_{p}(t)$ which does not necessarily coincide with the location of grid nodes on a fixed Eulerian mesh. A conservative extrapolation scheme is thus necessary to distribute $\mathbf{f}_{b}$ from the particle position onto the Eulerian grid which will be discussed later.

For a fully two-way coupled numerical simulation, we would have to take into account the spatial and temporal evolution of the local bubble concentration $C(\mathbf{x}, t)$ in the continuity equation. The continuity equation would then be expressed as $\partial_{t}(1-$ $C)+\partial_{j}\left[(1-C) U_{j}\right]=0[27]$. However, in our simulations we consider extremely low 
volume fractions of bubbles $(0.1 \%)$; the maximum instantaneous local volume fraction of bubbles is approximately ten times the global volume fraction $(\langle C(\mathbf{x}, t)\rangle \max \sim 1 \%)$ and in such a case the error in equation 1.2 is negligible. In cases of bubbly turbulent systems with higher global volume fractions, $C(\mathbf{x}, t)$ can be much higher than $1 \%$ where the bubbles may interact, coalesce, and can form dense groups of bubbles in the flow. In such a case the volumetric effect of the bubbles cannot be neglected any more.

\subsubsection{Dispersed phase}

The bubbles dispersed into the TC system are assumed to be clean, non-deformable and are tracked in a Lagrangian manner with effective forces such as drag, lift, added mass and buoyancy acting on them [25, 26, 42-44]. The generalised momentum equation for particles of density $\rho_{p}$ dispersed into a carrier fluid of density $\rho_{f}$ which takes into account the drag, lift, added mass and buoyancy forces reads:

$$
\begin{array}{r}
\rho_{p} V_{p} \frac{d \mathbf{v}}{d t}=\left(\rho_{p}-\rho_{f}\right) V_{p} \mathbf{g}-C_{D} \frac{\pi d_{b}^{2}}{8} \rho_{f}|\mathbf{v}-\mathbf{u}|(\mathbf{v}-\mathbf{u})+\rho_{f} V_{p} C_{M}\left(\frac{D \mathbf{u}}{D t}-\frac{d \mathbf{v}}{d t}\right) \\
+\rho_{f} V_{p} \frac{D \mathbf{u}}{D t}-C_{L} \rho_{f} V_{p}(\mathbf{v}-\mathbf{u}) \times \omega
\end{array}
$$

Based on the particle velocity, the position of each particle is updated according to

$$
\frac{d \mathbf{x}_{p}}{d t}=\mathbf{v}
$$

Since we consider clean spherical bubbles the effect of history forces is assumed negligible $[26,42,45]$. In equations (2.6) and (3.1), $\mathbf{u}$ is the velocity of the carrier phase at the particle position, $\mathbf{v}$ is the velocity of individual particle, $\mathbf{x}_{p}$ is the position of the particle, while $C_{D}, C_{M}, C_{L}$ are the drag, added mass and lift coefficients, respectively. Since we consider only very light particles (bubbles in water, i.e. $\rho_{p} / \rho_{f} \ll 1$ ), in this case equation (2.6) can be simplified into

$$
C_{M} \frac{d \mathbf{v}}{d t}=-\mathbf{g}-C_{D} \frac{\pi d_{p}^{2}}{8 V_{p}} \rho_{f}|\mathbf{v}-\mathbf{u}|(\mathbf{v}-\mathbf{u})+\left(1+C_{M}\right) \frac{D \mathbf{u}}{D t}-C_{L}(\mathbf{v}-\mathbf{u}) \times \omega
$$

In order to close equation (2.8), along with the coefficients for drag, lift and added mass (i.e. $C_{D}, C_{L}$, and $C_{M}$ ), information on the velocity, total acceleration and vorticity in the carrier phase is required at the exact location of the bubble. 
Again, since we assume that the dispersed phase is composed of clean spherical non-deformable bubbles, the value of the added mass coefficient is $C_{M}=1 / 2[45,46]$. In bubbly turbulent flows the exact value of the lift coefficient is not well known and could be a source of discrepancy in numerical studies. Climent et al. and Chouippe et al. $[24,25]$ use a lift coefficient which is dependent on both the bubble Reynolds number and the local shear intensity. By systematically varying the lift coefficient acting on the bubbles from 0 to 0.5 Sugiyama et al. [26] found that the value of the lift coefficient plays a crucial role in the mean bubble distribution. We use $C_{L}=1 / 2$, a simplified approach which has been used in many previous studies [26, 27, 42-44, 47]. Here, we note that our simulations by changing the value of the lift coefficient from $0.25-0.5$ resulted different trajectories of the bubbles but we find that the qualitative behaviour of the bubbles remains almost the same.

The drag coefficient $C_{D}$ is computed for each bubble individually and is dependent on the bubble Reynolds number defined as $R e_{b}=d_{p}|\mathbf{v}-\mathbf{u}| / \nu[45,48]$, namely

$$
C_{D}=\frac{16}{R e_{b}}\left[1+\frac{R e_{b}}{8+\frac{1}{2}\left(R e_{b}+3.315 \sqrt{R e_{b}}\right)}\right]
$$

The difference in the location of the bubbles and the grid nodes of the Eulerian mesh restricts us from calculating local flow quantities (i.e. velocity, total acceleration, and vorticity) directly from the carrier phase solution. Since the velocities are in three different positions in a staggered grid arrangement, all the three velocities belonging to any specific computational cell is first interpolated to the cell centre using the values from the nearest grid points. A tri-linear interpolation scheme, containing information from all the cell-centres surrounding a specific particle is used to calculate the carrier phase velocity at the exact particle position. The same approach is employed for the interpolation of the total acceleration and the vorticity in the flow.

In addition to interpolating local flow quantities, the back-reaction force from the bubbles (equation 2.5) needs to be extrapolated to the surrounding grid nodes. The back-reaction force from the particle $\mathbf{f}_{b}$ is extrapolated to the surrounding grid nodes residing in fixed computational volume (which is smaller than the bubble volume) using an exponential distribution function, which decreases monotonically with the distance between the grid nodes and the particle position. In the context of simulating particle-laden flows such an approach has already been discussed in detail by Capecelatro et al. [49]. The extrapolation scheme ensures second-order accuracy, conservation of the point back-reaction force while extrapolating the data from a Lagrangian location to a Eulerian mesh and is also stable when a non-uniform grid distribution is used. Time integration of dispersed phase momentum equation is 


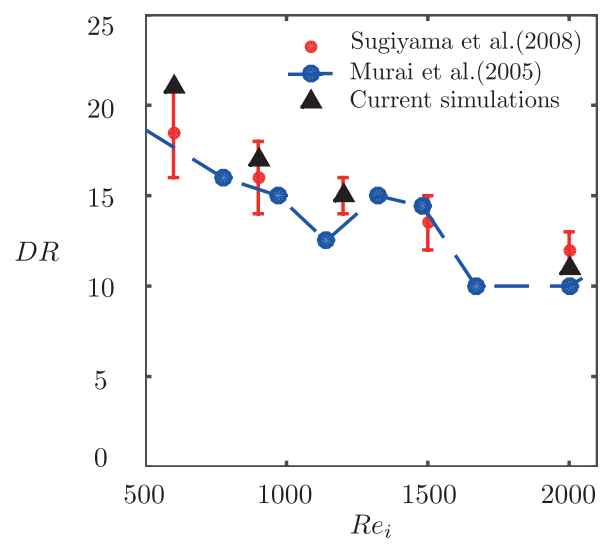

Figure 2.1: Percentage of drag reduction $(D R)$ as a function of the inner Reynolds number $R e_{i}$. The results from the current code are compared against the numerical simulations of Sugiyama et al. (2008) and experimental measurements of Murai et al. (2005). The global volume fraction of bubbles $\alpha_{g}=0.125 \%-0.670 \% ; F r=0.3-1.0$.

done by a second-order accurate Runge-Kutta scheme. The code has been parallelised using MPI and OpenMP directives and has a strong scaling of up to 1000 cores.

In order to validate the two-phase code, we perform simulations with the exact resolutions and parameters as done in Sugiyama et al. [26] and compare our results in Figure 2.1. Very good agreement is seen between the results of the current simulations, the simulations of Sugiyama et al. [26] and also the experiments of Murai et al. [18] where the drag reduction $(D R)$ is plotted against the inner cylinder Reynolds number. For the rest of the cases simulated in this chapter the parameters chosen are as follows: The geometry of the TC system is fixed by fixing the radius ratio $\eta=0.833$ and the aspect ratio to $\Gamma=4$. The global volume fraction $\alpha_{g}$ is fixed to $0.1 \%$, the relative bubble diameter to $d_{b} / d=0.01$, while the Froude number $F r$ of the bubbles is varied from 0.16 to 2.56 .

To reduce the computational cost, the simulation is initially run without any dispersed phase and once the flow in the single phase reaches a statistically stationary state, bubbles are placed at random positions throughout the domain. The velocity of these bubbles is equated to that of the velocity of the carrier phase interpolated at the bubble location and the two-phase flow is allowed to develop. Once the bubbles are introduced into the flow, the previously developed statistically stationary state of the single phase flow is disrupted and transients develop in the two-phase flow which eventually die out after approximately 100 full cylinder rotations. Additionally, it has been ensured that the mean axial velocity of the carrier phase in the domain is 


\begin{tabular}{c|c|c|c|c}
$R e_{i}$ & $N_{r} \times N_{\theta} \times N_{z}$ & $\Delta r^{+}$ & $d_{b} / \delta_{\nu}$ & $S t=\tau_{b} / \tau_{\eta}$ \\
\hline \hline 2500 & $180 \times 100 \times 150$ & 0.125 & 0.55 & 0.02 \\
3500 & $200 \times 100 \times 180$ & 0.150 & 0.75 & 0.04 \\
5000 & $220 \times 120 \times 200$ & 0.150 & 1.0 & 0.07 \\
8000 & $258 \times 150 \times 220$ & 0.125 & 1.25 & 0.10 \\
\hline
\end{tabular}

Table 2.1: Numerical details of the simulations. First column is the operating Reynolds number of the inner cylinder, second column is the radial grid spacing near the inner wall, the third column shows the radial grid refinement near the inner cylinder, the fourth column shows the diameter of the bubble normalised by the viscous length scale and the fifth column is the bubble Stokes number for each case. These values are for a geometry with radius ratio $\eta=0.833$ with an aspect ratio $\Gamma=4$ and a rotational symmetry $n_{\text {sym }}=6$ in the azimuthal direction while the maximum value of the bubble size relative the radial grid refinement in all simulations is $d_{b} / \Delta r_{\min } \sim 3$.

equal to zero. Similar to the single phase system, azimuthal and axial periodicity is employed for the dispersed phase i.e. when the bubbles exit an azimuthal or axial boundary they are re-entered at the opposite boundary at the exact same location with the same velocity. This approach is obviously different from experimental studies $[4,18,19,50]$, where the bubbles are injected at the bottom and are collected at the top and can be a cause of discrepancy in the comparison between numerical and experimental data.

In the current simulations, through some additional test runs we have ensured that the chosen axial extent of the domain does not influence the results. Details on the Nusselt number for both single phase and two-phase case with $\mathrm{Fr}=0.16$ is compared for $\Gamma=4$ and $\Gamma=8$ in table 2.2. An elastic bounce model is used for the interaction of the bubbles with the inner and outer cylinder wall [24-26]. While modifying the elastic bounce interaction may result in a change in the local bubble concentration near the wall, small variations in the coefficient of restitution of bubble impact with the wall does not influence the qualitative behaviour of the motion of bubbles which we study in a later section. Additionally, since the bubbles are assumed to be Lagrangian points, there is a possibility of bubbles overlapping among each other. Owing to the very low global volume fraction in the system $\left(\alpha_{g}=0.1 \%\right)$, the fraction of bubbles overlapping onto each other when taking into consideration their physical size is less than $10^{-3}$, which we consider to be negligible.

The procedure for calculating the Nusselt number $N u_{\omega}$ for both the single phase 
and the two-phase systems is as follows. The simulation is run for at least 50 full cylinder rotations after statistical stationarity is achieved and convergence is ensured by comparing the mean azimuthal velocity profiles for 50 and 100 full cylinder rotations. Additionally it is ensured that the net angular velocity flux in the radial direction corrected with the volumetric forcing from the dispersed bubbles is constant. The torques on both the cylinders are then averaged in time and it is ensured that the difference in $\left\langle N u_{\omega}\right\rangle$ for both inner and outer cylinders is less than $1 \%$. Figure 2.2 shows a typical time series of the non-dimensional angular velocity transport $N u_{\omega}$ on both the inner and outer cylinder after the transients in the two-phase system die out and the system reaches a statistically stationary state. The net percentage drag reduction $(D R)$ is then calculated according to equation (2.4). To maintain a global volume fraction of $\alpha_{g}=0.1 \%$ with bubbles of size $d_{b} / d=0.01$, approximately 50000 bubbles are required in the simulation. In order to calculate the effective forces acting on the point-wise particles according to equation (2.8), and advect them with sufficient accuracy, the size of the bubble should be of the same order as the smallest relevant length scale in the carrier phase. The ratio of bubble diameter $\left(d_{b}\right)$ to the viscous length scale $\delta_{\nu}$ is of the order of 1 for all the simulations considered in this chapter. In table 2.1 we give details on the resolutions for various Reynolds number and the corresponding Stokes number of the bubbles $\left(S t=\tau_{b} / \tau_{\eta}\right.$, where $\tau_{b}=d_{b}^{2} / 24 \nu$ is the bubble response time and $\tau_{\eta}$ is the Kolmogorov time scale). In the next section we discuss the main results of this chapter, where we study the effect of the operating Reynolds number $R e_{i}$, and the Froude number $F r$ on the global response of the two-phase TC system and also on the local flow dynamics.

\subsection{Results}

\subsubsection{Drag reduction}

We first focus on the change in the global response of the TC system with the addition of the dispersed phase. In figure 2.3(a) we compare the friction coefficient $C_{f}$ for the single phase and two-phase cases for five different Froude numbers $F r=0.16$, $0.32,1.28,2.56$ and 1000. When $F r<1$ and in particular for low $R e_{i}$, we observe a reduction in the friction factor $C_{f}$ for two-phase case as compared to the single phase case. In order to observe this difference more clearly we compute the net percentage drag reduction $D R$ according to equation 2.4. It is shown as function of $R e_{i}$ for the five different $F r$ in figure 2.3(b). Almost $4 \%$ drag reduction is achieved at $R e_{i}=2500$ and $F r=0.16$. For fixed and small enough $R e_{i}$, increasing the $F r$ number of the 


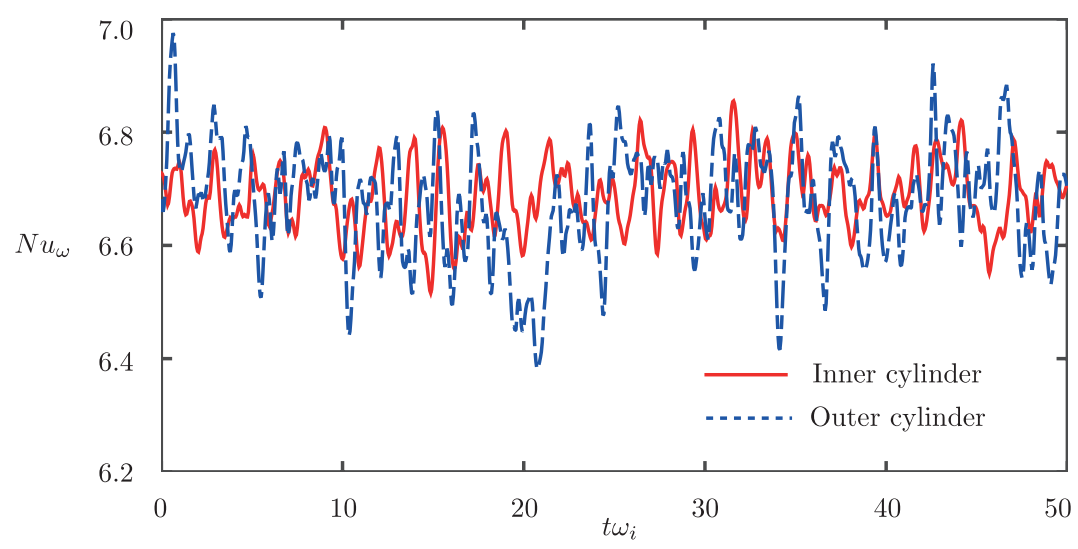

Figure 2.2: Typical time series of the instantaneous torques at the inner (solid red line) and outer cylinder (dashed blue line). $R e_{i}=2500, F r=0.16, \alpha_{g}=0.1 \%$ Temporal origin is arbitrary and after the system has achieved statistically stationary state.

(a)

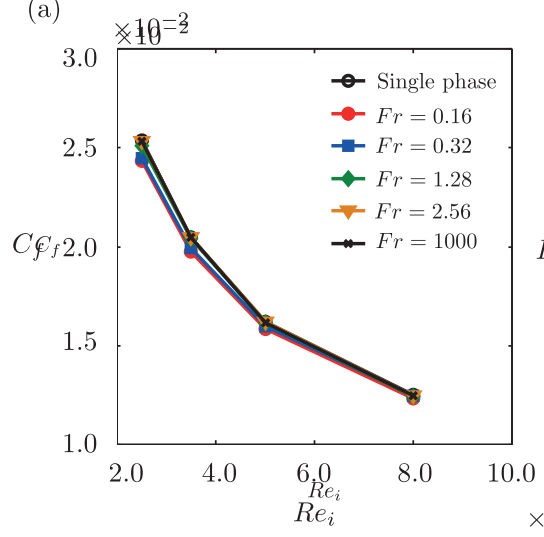

(b)

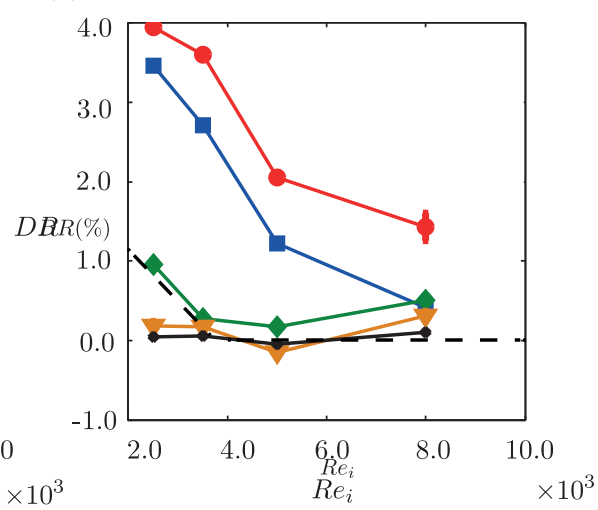

Figure 2.3: (a) Friction factor $C_{f}\left(R e_{i}\right)$ for single phase TC flow and for bubbly TC flow with bubbles of five different Froude numbers. Only for $F r<1$ drag reduction occurs. This drag reduction is much better expressed in terms of the percentage of drag reduction $(D R)$, which is plotted in (b), again as a function of the inner Reynolds number $R e_{i}$. Positive $D R$ indicates that the driving torque in the two-phase case is lower than that of the single phase case. The dashed line shows the path taken by $R e_{i}-F r$ number in the experiments by Murai et al. (2008). 


\begin{tabular}{c|ccccc|cc}
$R e_{i}$ & Single phase & $F r=0.16$ & $F r=0.32$ & $F r=1.28$ & $F r=2.56$ & $\begin{array}{c}\text { Single phase } \\
\Gamma=8\end{array}$ & $\begin{array}{c}F r=0.16 \\
\Gamma=8\end{array}$ \\
\hline \hline 2500 & 6.94 & 6.66 & 6.70 & 6.87 & 6.93 & 6.93 & 6.67 \\
3500 & 7.85 & 7.57 & 7.64 & 7.83 & 7.84 & 7.86 & 7.58 \\
5000 & 8.85 & 8.66 & 8.74 & 8.83 & 8.86 & 8.84 & 8.66 \\
8000 & 10.93 & 10.78 & 10.89 & 10.88 & 10.90 & 10.93 & 10.78 \\
\hline
\end{tabular}

Table 2.2: Nusselt numbers for the single phase and two-phase cases for different $R e_{i}$ and $F r$ numbers. Last two columns show the Nusselt numbers with aspect ratio $\Gamma=8$.

bubbles leads to a decrease in the drag reduction. Vice versa when the $F r$ number is kept fixed the drag reduction $D R$ gets less for increasing $R e_{i}$. This holds in particular for configurations with $F r<1$, i.e., when the buoyancy of the bubbles is dominant over the driving of the system. With $F r>1$, for at least within the examined $R e_{i}$ range, there is overall no systematic trend with $R e_{i}$ and negligible drag reduction is observed, thus indicating that bubble buoyancy has a strong role in achieving drag reduction.

A unique difference in these results when compared to the experimental study of Murai et al. [19] is that here we have explicit control over the $F r$ number, i.e. independently of the Reynolds number $R e_{i}$. Murai et al. [19] found almost negligible drag reduction beyond $R e_{i}=3000$; however in their setup the $F r$ of the bubbles is dependent on $R e_{i}$ and thus at $R e_{i}=2000$ the $F r$ number was already above one. The path taken by the $R e_{i}-F r$ number in the experiments is shown in figure 2.3(b) by a dashed line. In the current simulations, in contrast, we observe that by fixing the $F r$ number of the bubbles to less than one, drag reduction can be observed even at $R e_{i}=8000$. The details on the Nusselt number for the single phase and two-phase cases for these simulations and also reference data for the simulations with a larger aspect ratio $(\Gamma=8)$ is given in table 2.2 .

It can be expected that at the asymptotically large value of $F r=1000$, the centripetal acceleration can play the role of buoyancy for bubbles immersed in a horizontal channel flow $[11,13,16,51]$ which may lead to drag reduction. However, we do not find any such drag reduction in the TC system as seen in figure 2.3 and we think this might be due to the different physical mechanisms governing drag reduction in both systems. In comparison with the current simulations, experiments with larger bubbles and thus higher gas volume fractions $\left(d_{b}^{+}>>1, \alpha_{g}>1 \%\right)$ have been performed in (bubbly) turbulent channel flows to obtain sustainable levels of drag reduction $[11,13]$. In experiments with smaller bubbles and lower gas volume 
(a)

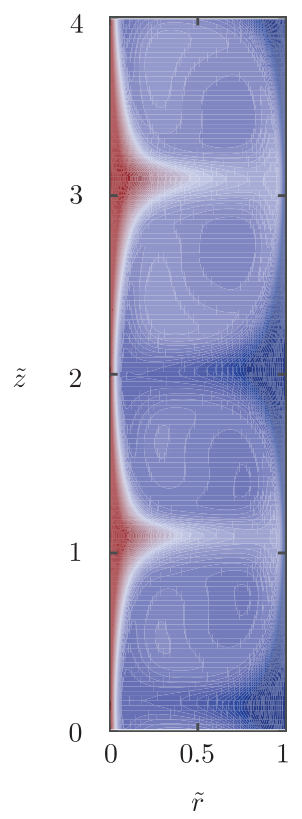

(b)

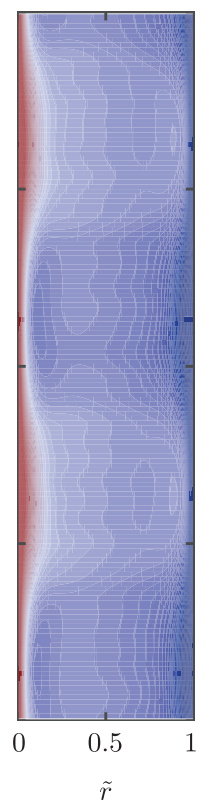

(c)

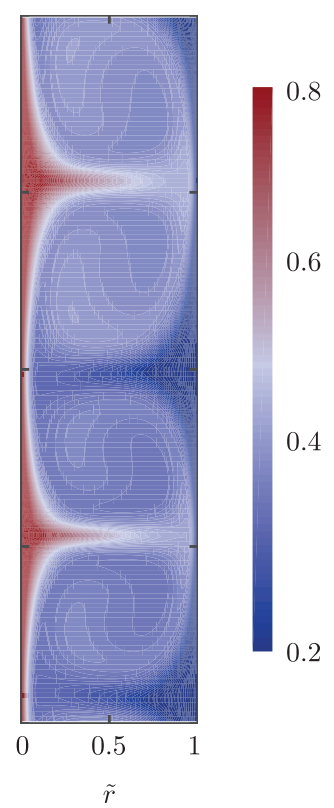

Figure 2.4: (Colour online) Contour plots of azimuthally- and time averaged azimuthal velocity field $\left\langle\bar{u}_{\theta}\right\rangle_{\theta, t}$ for a fixed $R e_{i}=2500$ (a) Single phase (b) Two-phase flow with $F r=0.16$ and (c) with $F r=1.28$. For the low $F r$ case the Taylor rolls are considerably weakened by the strongly buoyant bubbles.

fractions $\left(d_{b}^{+} \sim 1, \alpha_{g} \sim 1 \%\right)$, Pang et al. [16] and Harleman et al. [51] found extremely small levels of drag reduction which is similar to what we find in our simulations. Additionally, the drag reduction effect observed in Taylor-Couette flows in the weakly turbulent regime is through disruption of coherent Taylor rolls which are fixed in space and time for single phase flows and are responsible for the majority of the angular momentum transport. In contrast, for channel flows co-spectra analysis shows that these wall-attached large scale structures are inactive [52].

\subsubsection{Carrier phase velocity fields}

In figure 2.4, we compare the contour plots of the azimuthal velocity, averaged in the azimuthal direction and over time. The Reynolds number is fixed at $R e_{i}=2500$ and averaged contours of two systems with $F r=0.16$ and $F r=1.28$ is compared with that 
(a)

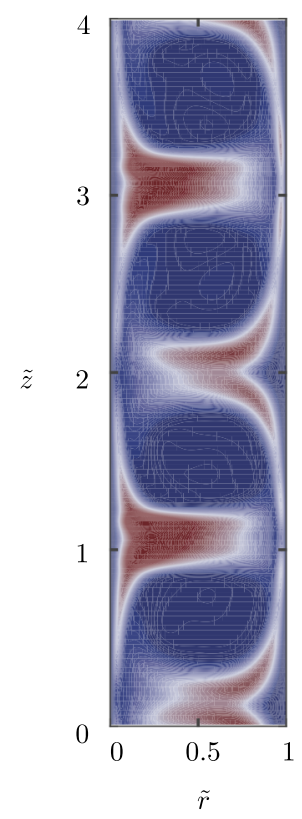

(b)

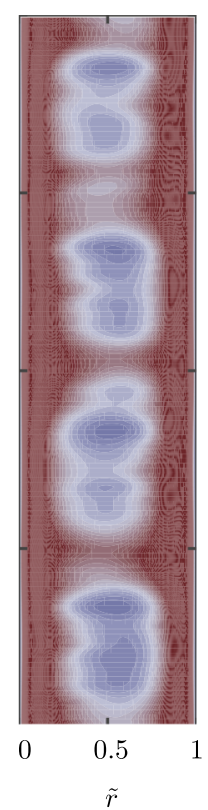

(c)

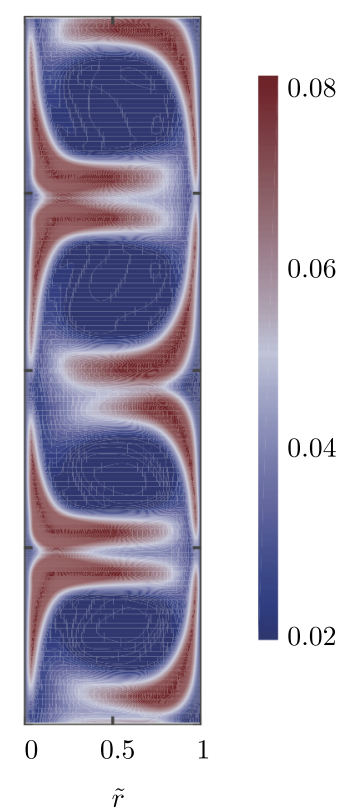

Figure 2.5: Contour plots of azimuthal and time averaged r.m.s. of the velocity fluctuations $\left(u_{\theta}^{\prime}\right)$ for a fixed at $R e_{i}=2500$; (a) Single phase (b) Two-phase flow with $F r=0.16$ and (c) with $F r=1.28$. Same colour bar is used for all three plots. Again, the effect of the strongly buoyant bubbles $(F r=0.16)$ on the Taylor rolls can be clearly observed.

of the single phase flow. As mentioned earlier (also see again figure 2.3), the drag reduction $D R$ decreases with increasing Froude $F r$ for fixed $R e_{i}$. In figure 2.4(c) $(F r=1.28)$, a clear signature of the Taylor vortex can be observed with a structure very similar to that of the single phase case as seen in figure 2.4(a). When $F r<1$ (figure 2.4b) a strong footprint of the Taylor vortex is not observed any more. The strong buoyancy of the bubbles as compared to the driving of the system (i.e. $F r<1$ ) is responsible for disrupting the Taylor vortices, which have concentrated regions of high strain-rates and are thus highly dissipative [26]. With increasing $F r$, the Taylor vortex structure resembles more to that of a single phase system [53], resulting in a drop in $D R$.

This is also observed in figure 2.5 where we show contour plots of azimuthally and time averaged r.m.s. of the azimuthal velocity fluctuation $u_{\theta}^{\prime}$. These are computed 
(a)

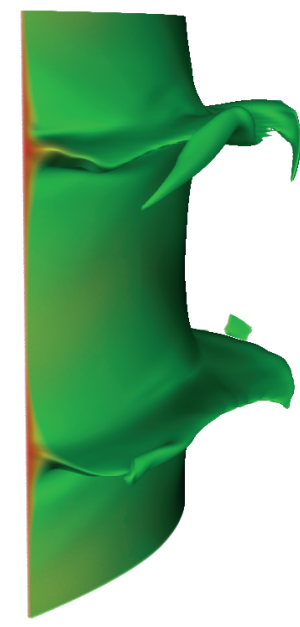

(b)

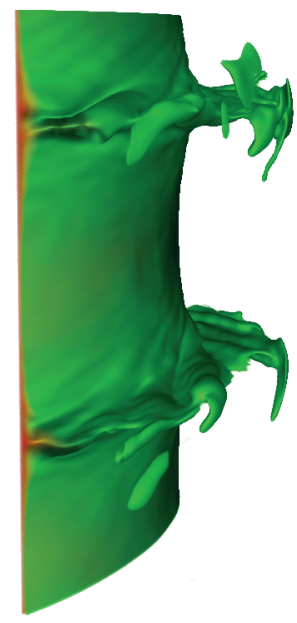

(c)

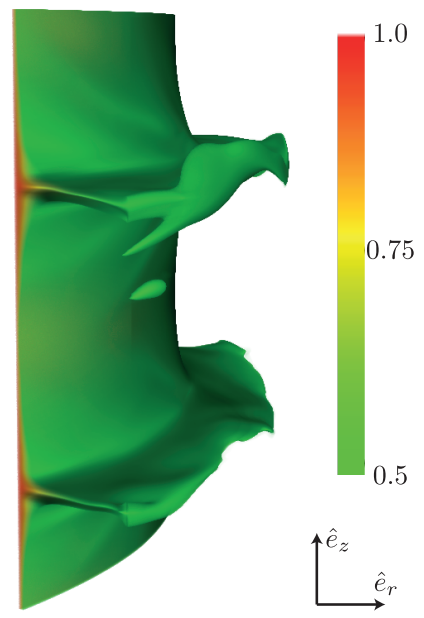

Figure 2.6: Three dimensional instantaneous snapshot of the azimuthal velocity field $u_{\theta}$ for a fixed $R e_{i}=2500$ (a) Single phase (b) $F r=0.16$ (c) $F r=1.28$.

in form of the root mean squared (r.m.s.) value as $u^{\prime}(r, z)=\left[\left\langle u^{2}\right\rangle_{\theta, t}-\langle u\rangle_{\theta, t}^{2}\right]^{\frac{1}{2}}$. For the single phase flow (figure 2.5(a)), there exist two localised regions near the inner-cylinder which show a peak in the velocity fluctuations. These regions are associated to the sites of plume ejection [53-55]. In a two-phase system with strong buoyant bubbles $(F r<1$ i.e. figure $2.5(\mathrm{~b}))$ there is a peak in the fluctuation along the complete axial extent of both the inner and outer cylinders. The bubbles rising near the walls of the cylinders by virtue of their buoyancy are responsible for such a behaviour. However, unlike in the single phase case where the fluctuations extend into the gap width, in the two-phase case with $F r<1$ they are localised near the cylinder walls giving an indication that the plumes are much weaker. This is not observed in the case where the bubbles are weakly buoyant $(F r>1$, i.e. figure $2.5(\mathrm{c}))$. Instead, we see that the axial extent of the plume ejection site is extended when compared to the single phase system. This is a result of bubbles being captured by the Taylor vortices at the sites of plume ejection after sliding on the inner cylinder for a short period. For a single phase flow, the plumes ejected on the inner cylinder wall are responsible for majority of the angular velocity transport. Additionally, they assist in the transition to the ultimate state of turbulence where the turbulence is fully developed in both bulk and boundary layers [55]. 
(a)

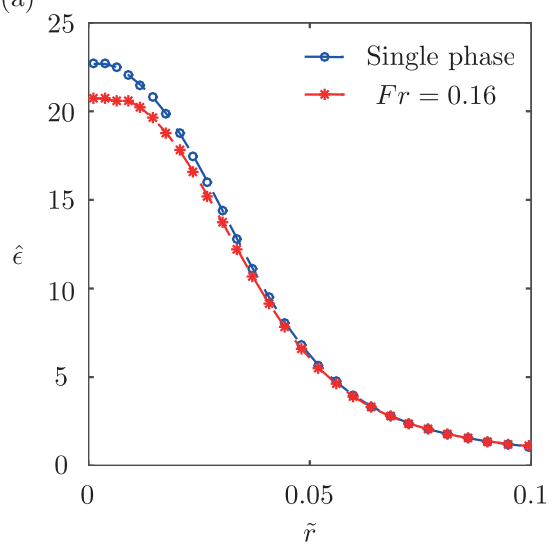

(b)

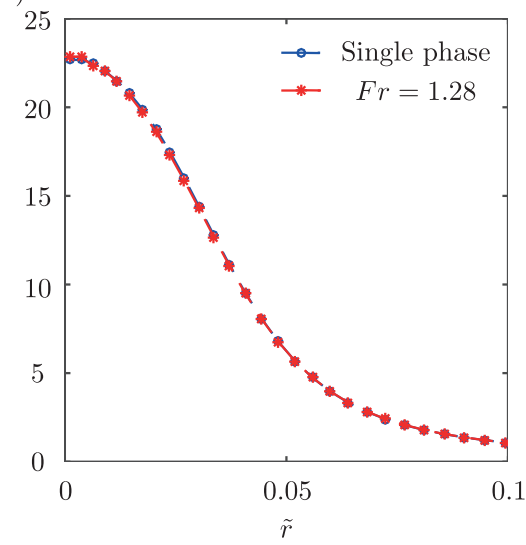

Figure 2.7: Comparison of azimuthal, axial and time averaged viscous dissipation profiles near the inner cylinder wall. The $R e_{i}$ in both (a) and (b) is 2500 . For the two-phase system (a) $F r=0.16$ (b) $F r=1.28$

In figure 2.6 we show three dimensional instantaneous snapshots of the azimuthal velocity field for the single phase case and the two-phase cases for both $F r=0.16$ and $F r=1.28$. The important observation made here is that while the ejection of plumes from the inner cylinder $(\tilde{r}=0)$ is very strong for the single phase case and $F r=1.28$, it is much weaker for $F r=0.16$. The strong buoyancy of the bubbles $(F r=0.16)$ causes the plume ejection region to sweep the inner cylinder surface and thus when averaged in time a clear footprint of the ejection region is not observed any more (c.f. figure 2.4b).

In order to show clearly how the bubbles affect these plumes, in figure 2.7 we plot the viscous dissipation profile near the inner cylinder wall. The total dissipation per unit mass is calculated as a volume and time average of the local dissipation rate, and as shown by Eckhardt et al. [36] is directly related to the driving torque. The viscous dissipation is normalised accordingly $\hat{\epsilon}=\epsilon / \nu\left(U_{i} / d\right)^{2}$ and in figure 2.7, we compare the dissipation profiles of a two-phase system with strongly buoyant bubbles $(F r<1)$ and weakly buoyant bubbles $(F r>1)$ with a single phase system. When $F r<1$, the viscous dissipation is lower than that compared to the single phase and difference can be clearly observed in figure 2.7(a). This is not the case in figure 2.7(b), where the difference is almost negligible and thus also resulting in minimal drag reduction. Such a behaviour is related to the pattern of distribution of bubbles near the inner cylinder and is discussed in a later section. In addition to a drop in $D R$ with increasing $F r$ 
(a)

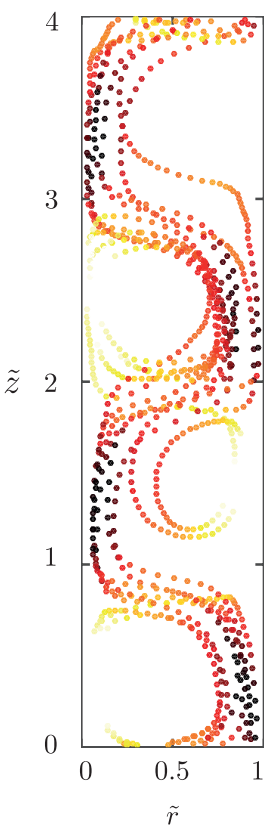

(b)

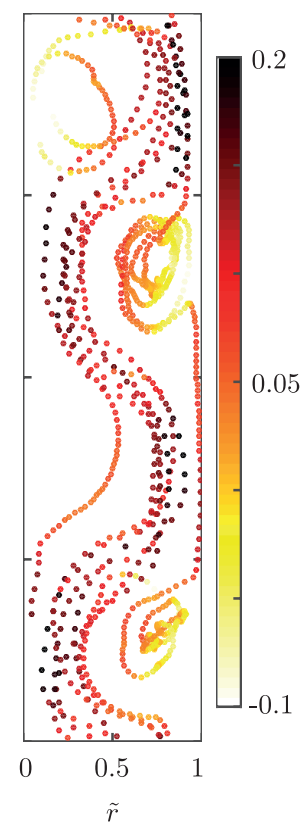

(c)

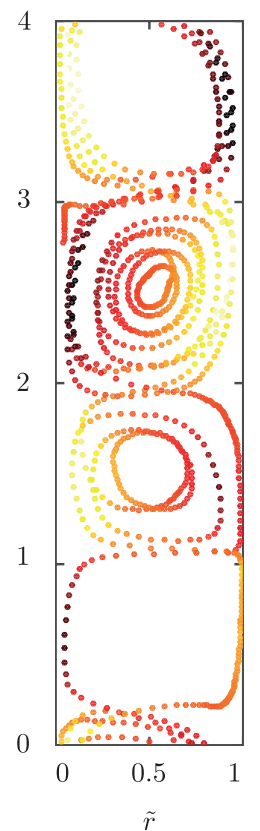

(d)

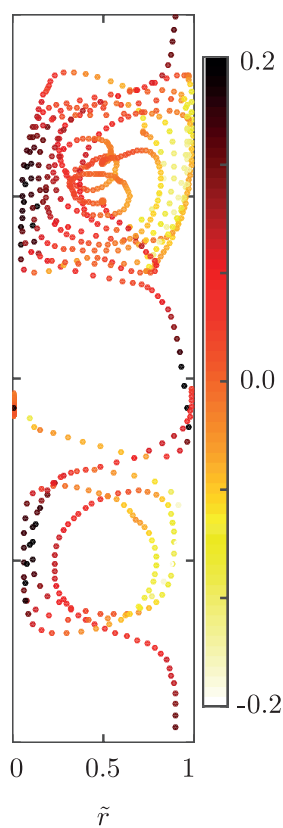

Figure 2.8: Full trajectory of a single bubble in the $r-z$ plane of the two-phase TC system. (a) $R e_{i}=2500, F r=0.16$ (b) $R e_{i}=5000, F r=0.16$ (c) $R e_{i}=2500, F r=1.28$ (d) $R e_{i}=5000$, $F r=1.28$. The bubble is tracked for almost 20 full inner cylinder rotations in all cases after the system has reached statistically stationary state. Same colour-scale is used for cases with the same $\mathrm{Fr}$ numbers and indicates the axial velocity $v_{z}$ of the bubbles. While the less buoyant bubbles $(F r=1.28,(\mathrm{c}),(\mathrm{d}))$ tend to get trapped in the Taylor rolls, the more buoyant ones $(F r=0.16$, (a),(b)) rise through and weaken them.

at a fixed $R e_{i}$, we also notice a drop in $D R$ with $R e_{i}$ even for bubbles with $F r<1$. With increase in $R e_{i}$, the coherent flow structures become less responsible for the angular velocity transport $[53,55]$. The strong buoyancy effect of the bubbles (i.e. $\mathrm{Fr}<1$ ) thus acts on weaker coherent structures and lose their efficiency on affecting the angular velocity transport in the system. This trend was also observed in the experiments by Murai et al. $[18,19]$ who found that the $D R$ dropped to almost zero at $R e_{i}=4000$. We show that although there is a drop in $D R$ with increasing $R e_{i}$, little $D R$ can be attained even at $R e_{i}=8000$ provided $F r<1$. 


\subsubsection{Motion of dispersed phase}

In this section we look at the different trajectories of bubbles for different $R e_{i}$ and Fr numbers. In figure 2.8, we show four different trajectories of an individual bubble for two different $R e_{i}$ and $F r$ numbers, respectively. There is a strong azimuthal motion imposed on the bubbles by the carrier flow while their interaction with the underlying coherent Taylor rolls can be easily visualised in the $r-z$ plane as has been done in figure 2.8. For strong buoyant bubbles i.e. $F r<1$ (c.f. figure 2.8a), the bubble primarily has an upward motion moving through different Taylor vortices as expected. For higher $R e_{i}$ where the turbulence is more developed and intense, the bubbles have a tendency to get trapped in the small vortical structures inside the large scale Taylor rolls. This can be observed at an axial height of $\tilde{z} \simeq 0.5$ and $\tilde{z} \simeq 2.5$ in the right panel of figure 2.8a. Also for larger $F r$ number (here $F r=1.28$ ) at fixed $R e_{i}=2500$ as shown in the left panel of Figure $2.8 \mathrm{~b}$, the bubbles have a tendency to be trapped inside the Taylor vortex (in this case at a height of $\tilde{z} \simeq 3.0$ ) while rising along the inner cylinder wall. This behaviour has been observed also in the experiments by Murai et al. [19] and Fokoua et al. [50]. The influence of such a motion can be seen in the contour plots of the velocity fluctuations (cf. figure 2.5(c)). When the $R e_{i}$ is increased to 5000 and $F r>1$, the smooth structured motion of the bubble inside the Taylor vortex as seen in $R e_{i}=2500$ is lost. Due to increased levels of turbulence in addition to loss of importance of coherent structures the bubble motion is more erratic as compared to the previous case. For both $R e_{i}$, a clear difference can be observed in the trajectories of the bubble depending on whether $\mathrm{Fr}>1$ or $\mathrm{Fr}<1$. For low $R e_{i}$ a bubble which smoothly passes through each Taylor vortex tends to get trapped with increase in the $F r$ number; while for a higher $R e_{i}$ the bubbles which spend short periods of time in small scale vortices moves in a more erratic manner with increasing $F r$. In all the above cases, it is to be noted that there is also a strong azimuthal motion of bubbles.

As observed in figure 2.8, the bubbles dispersed into the TC system exhibit various kinds of motion such as sliding along the inner cylinder wall, outer cylinder wall, organised or erratic motion in the Taylor vortices. In order to understand and categorise such bubble motion more precisely, it would be useful to look at the mean radial and axial distribution of the bubbles and also their corresponding Reynolds numbers. For this purpose we divide the domain into three different regions (i) inner boundary layer (IBL) (ii) bulk region (BULK) and (iii) outer boundary layer (OBL). The method of calculating the inner and outer boundary layer thickness is described in Ostilla-Monico et al. [53, 55] which we discuss here in brief. A straight line is first fitted through the first three computational grid points near the inner and respective 
outer cylinder wall. For the bulk a straight line is fitted through the point of inflection and the two nearest grid points. The intersection of the line drawn through the bulk and the lines near the inner and outer cylinder wall give the inner and outer boundary layer thickness, respectively.

In figure 2.9, we show normalised probability distribution functions (PDF) of the Reynolds number of the bubbles $\left(R e_{b}\right)$ in the three different regions of the domain as described above. When the Froude number of the bubbles is less than one, $R e_{b}$ has a comparatively narrow distribution as compared to higher Froude number systems, regardless of the operating Reynolds number $R e_{i}$ of the inner cylinder and also of the position of the bubbles in the domain. When $F r<1$, the bubbles primarily have an upward drifting motion through the Taylor rolls (cf. figure 2.8) independent of their position in the domain which is reflected in these histograms. However for increasing inner cylinder Reynolds number $R e_{i}$ the PDF becomes more symmetric as compared to lower $R e_{i}$ which is a result of bubbles getting trapped in the intense vortical structures (also seen in the right panel of figure 2.8 (a)). The distribution of $R e_{b}$ becomes wider for higher $F r$ numbers (less buoyancy); more in the inner boundary layer than the outer boundary layer. This is a result of combination of two events; namely the ejection of plumes from the inner cylinder where the bubbles are trapped before being pulled into the Taylor roll and a relatively stronger component of the azimuthal slip velocity near the inner cylinder as compared to the outer cylinder where it is close to zero.

Now that we have looked at how the motion of bubbles depends on the $R e_{i}$ and Fr numbers, in the next part we study the mean distribution of the bubbles in the domain. In Table 2.3 we show the percentage volume fraction of bubbles accumulated in the inner boundary layer $\left(\alpha_{i}\right)$, outer boundary layer $\left(\alpha_{o}\right)$, and the bulk $\left(\alpha_{b}\right)$. It is clear that the percentage of bubbles accumulated in the inner boundary layer is highest and for all cases it increases with $F r$. With increasing $F r$ the bubbles initially residing in the bulk and the outer boundary layer now migrate to the inner cylinder wall. Also the percentage distribution of the bubbles between the three different regions $\left(\alpha_{i}, \alpha_{b}\right.$, and $\left.\alpha_{o}\right)$ does not change significantly for a fixed $F r$ number with increasing the $R e_{i}$ number.

In figure 2.10 we show the azimuthally, axially and time averaged radial profiles of the bubble volume fraction for $R e_{i}=2500$ and 5000 which gives a clear indication of accumulation of bubbles near the inner cylinder wall independent of the $R e_{i}$ number. The local volume fraction of the bubbles near the inner cylinder wall (inset of figure 2.10 ) is higher for $F r=0.16$ as compared to $F r=1.28$. But table 2.3 shows that there is a higher percentage of bubble accumulation near the inner cylinder wall for $F r=1.28$, 

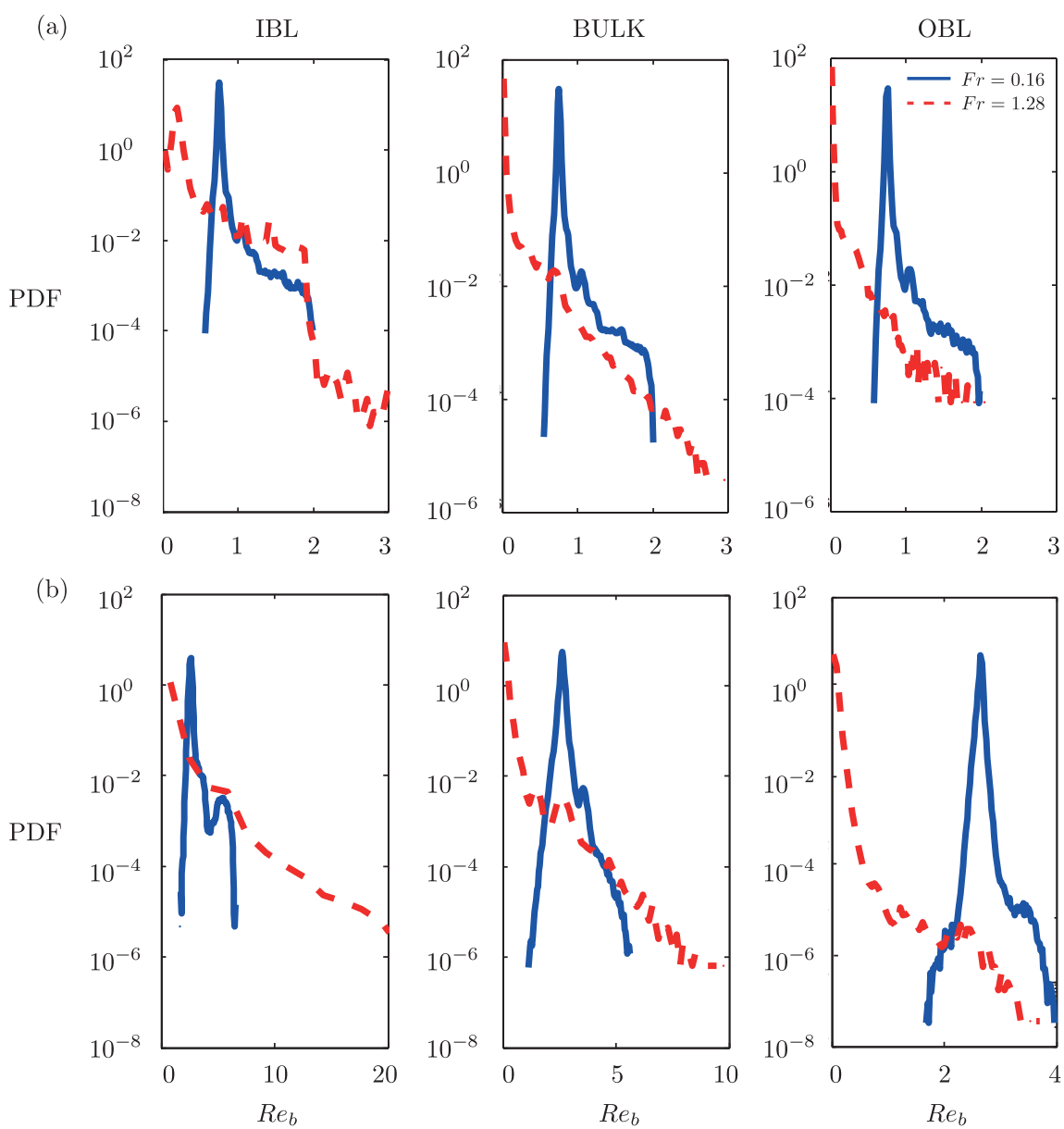

Figure 2.9: Probability distribution functions (pdf's) of the bubble Reynolds number for (a) $R e_{i}=$ 2500 (b) $R e_{i}=5000$. Left panels corresponds to the inner boundary layer region (IBL), middle panels to the bulk region (BULK), and right most panels to the outer boundary layer region (OBL). 


\begin{tabular}{cc|c|c|c}
$R e_{i}$ & $F r$ & $\alpha_{i}(\%)$ & $\alpha_{b}(\%)$ & $\alpha_{o}(\%)$ \\
\hline \hline 2500 & 0.16 & 0.2372 & 0.0927 & 0.0771 \\
2500 & 1.28 & 1.1363 & 0.0346 & 0.0327 \\
5000 & 0.16 & 0.2216 & 0.0961 & 0.0675 \\
5000 & 1.28 & 1.0822 & 0.0555 & 0.0463 \\
\hline
\end{tabular}

Table 2.3: Percentage volume fraction of bubbles in the inner boundary layer $\left(\alpha_{i}\right)$, bulk $\left(\alpha_{b}\right)$, and outer boundary layer $\left(\alpha_{o}\right)$ for two $\operatorname{Re} e_{i}$ and $\operatorname{Fr}$ numbers.
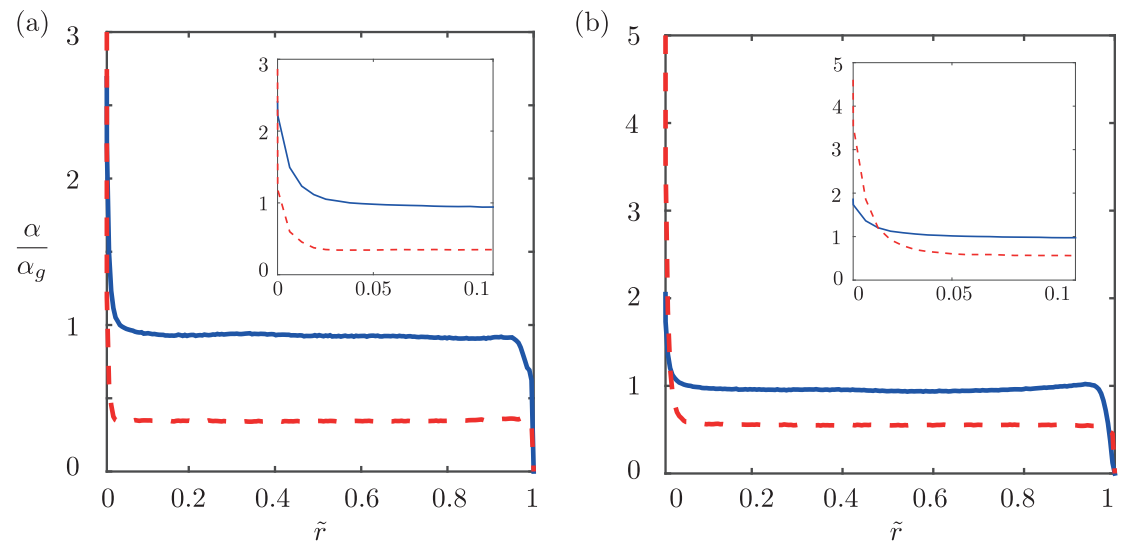

Figure 2.10: Radial profiles of the azimuthally, axially and time averaged local bubble volume fraction ( $\alpha$ ) for (a) $R e_{i}=2500$ (b) $R e_{i}=5000$. Solid blue lines refer to $F r=0.16$, while the dashed red lines refer to $F r=1.28$. The insets show the same profile near the inner cylinder wall. $\alpha$ is normalised with $\alpha_{g}=0.1 \%$, which is the global volume fraction in this case. 

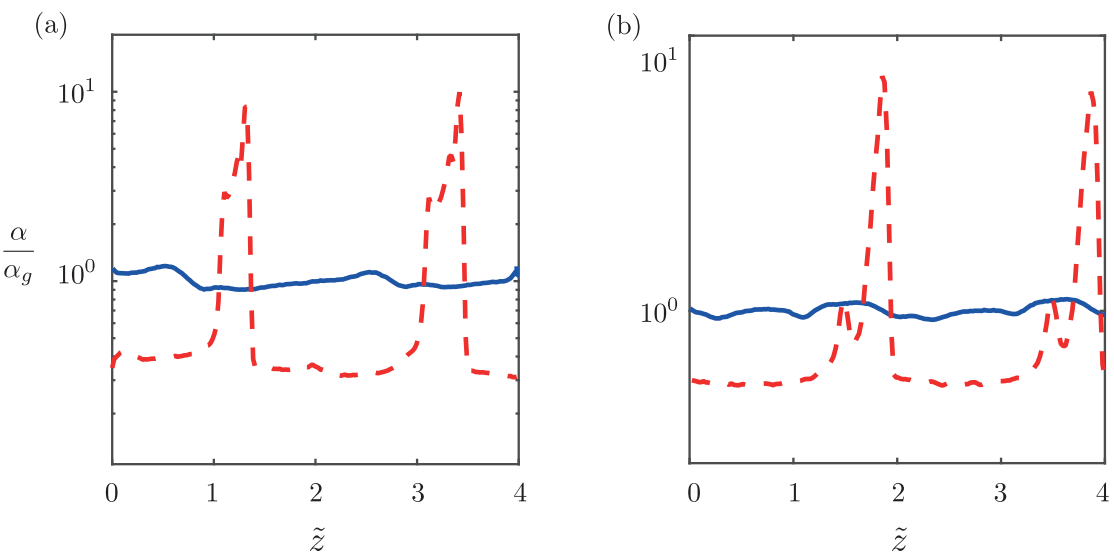

Figure 2.11: Axial profiles of the azimuthally, radially and time averaged local bubble volume fraction ( $\alpha$ ) for (a) $R e_{i}=2500$ (b) $R e_{i}=5000$. Solid blue lines refer to $F r=0.16$, while the dashed red lines refer to $F r=1.28$. $\alpha$ is normalised with $\alpha_{g}=0.1 \%$, which is the global volume fraction in this case. Again, the trapping of the less buoyant bubbles in the Taylor rolls becomes evident (larger Fr).

which means that there is a higher percentage of bubbles sticking to the inner cylinder wall. When $F r=0.16$, the local volume fraction $(\alpha)$ in the bulk of the system is close to that of the global volume fraction $\left(\alpha_{g}\right)$. With increase in $F r$ number, the local volume fraction decreases in the bulk considerably, and more bubbles stick to the inner cylinder. These bubbles play a major role in influencing the dynamics of plume ejection and also near-wall viscous dissipation $(\hat{\epsilon})$ as seen in figure 2.5 and figure 2.7, respectively. While in the case of $F r<1$, the disruption of plumes and its consequence on weakening the Taylor rolls results in low-strain rate regions in the plume impacting regions, there is no such effect when $F r>1$ i.e. when the buoyancy of the bubbles is weaker.

Similar to figure 2.10 we also plot the axial distribution of bubbles in the axial direction in figure 2.11. The local volume fraction is averaged in the azimuthal, radial direction and in time. For strong buoyant bubbles $(F r=0.16)$, the axial distribution displays a largely uniform behaviour while for the increased $F r$ number there are two distinct peaks in the local volume fraction of the bubbles for both $R e_{i}$ numbers. The positions of these peaks correspond to the plume ejection sites (i.e the outflow region) on the inner cylinder wall and are an effect of bubbles accumulating near these sites, before they are advected into the Taylor vortices. This distribution of bubbles when $F r>1$ is similar to the spiral pattern of bubbles observed in the experimental results of Murai et al. $[18,19]$ when the Froude number is higher than one and the operating 
Reynolds number of the inner cylinder is $R e_{i}=2100-4500$.

\subsection{Summary}

The drag reduction in turbulent bubbly TC flow compared to the corresponding single phase system depends on various control parameters such as the operating Reynolds number, the diameter of the bubbles, the relative strength of buoyancy compared to the driving and the strength of coherent vortical structures. It is extremely challenging to independently control each of these parameters in experiments and study its effect on the overall drag reduction. In this chapter we perform numerical simulations of a two-phase TC system using an Euler-Lagrange approach which allows us to track almost 50,000 bubbles simultaneously up to an operating Reynolds number of $R e_{i}=8000$ and also gives us the freedom to control various parameters independently. We find that the relative strength of the buoyancy as compared to the inner cylinder driving (quantified by the Froude number $\mathrm{Fr}$ ) is crucial to achieve drag reduction at higher Reynolds number. When the Froude number $F r$ is less than one, drag reduction was observed up to $R e_{i}=8000$, the largest $R e_{i}$ we studied here. When the $F r$ number is larger than one, almost negligible drag reduction was observed for all $R e_{i}$. We also observe from the averaged azimuthal velocity fields that for a fixed $R e_{i}=2500$, the coherent Taylor roll is much weaker for $F r=0.16$ due to the rising strongly buoyant bubbles, as compared to $F r=1.28$. The weakened Taylor rolls in turn imply drag reduction. The effect of the bubbles on the Taylor rolls is also observed in the contour plots of the azimuthal velocity fluctuations. By analysing the individual trajectory of the bubbles, we showed that while for a low $R e_{i}=2500$ the bubbles have primarily either a upward motion (lower $F r$ ) or coherent motion inside the Taylor roll (larger $F r$ ). Similar behaviour was observed even in the case of a higher $R e_{i}$, but with a more erratic movement. 


\section{Chapter 3}

\section{Deformation and orientation statistics of sub-Kolmogorov neutrally buoyant drops}

Based on: Vamsi Spandan, Detlef Lohse, Roberto Verzicco, 'Deformation and orientation statistics of neutrally buoyant sub-Kolmogorov ellipsoidal droplets in turbulent Taylor-Couette flow' Journal of Fluid Mechanics, Vol. 809, 480-501, 2016 (arXiv id: 1606.03835) 


\subsection{Introduction}

Multi-phase turbulent flows are ubiquitous in nature and several industrial applications such as cloud formation, plankton dispersion in sea, fuel sprays, emulsification, homogenisation and mixing in chemical reactors and drag reduction. In particular, two-phase flows which typically comprise a secondary phase dispersed into a primary carrier flow (e.g. droplets/bubbles dispersed into water or air) are prevalent in many applications involving drag reduction, heat transfer, liquid atomisation etc. Among the many parameters that influence the global response and local flow dynamics of two-phase systems, the shape, deformability and orientation of a non-spherical anisotropic dispersed phase plays a very important role. Understanding the dynamics of deformation, orientation of an anisotropic dispersed phase (for e.g. ellipsoidal bubbles or drops) and its dependence on the underlying local flow topology can thus be useful for optimising several industrial and engineering applications.

In this chapter, we study the shape and orientation statistics of neutrally buoyant, sub-Kolmogorov droplets dispersed into a turbulent Taylor-Couette (TC) flow. TC flow is one of the paradigmatic systems to study turbulence in wall-bounded systems (see $§ 1)$. In the last decade, two-phase TC flow has received massive interest due to the strong reductions in drag that can be achieved with the addition of a very small concentration of dispersed phase $(\sim O(1) \%$ of bubbles in water $)$. Moreover, through several experimental and numerical studies in turbulent channel and TC flow, it has been shown that the deformability of the bubbles is of paramount importance to achieve strong drag reduction $[4,14,15]$ while the exact physical mechanism is still unclear. It thus becomes important to understand the dynamics of deformation of a dispersed phase (for example bubbles/drops) in a turbulent carrier flow.

The degree of deformation of the dispersed phase depends on many factors such as the size of the drops/bubbles, intensity of local inertial and viscous forces which tend to elongate or deform the drop from its initial spherical shape, surface tension forces which are responsible for restoring the drop back to its original spherical shape etc. $[56,57]$. In his pioneering work, Taylor et al. $[58,59]$ was one of the first to study theoretically the deformation of a viscous drop dispersed into a carrier fluid where he showed that the shape of a deformed drop in presence of a velocity gradient depends on the viscosity ratio of the droplet to the carrier phase $\hat{\mu}=\mu_{d} / \mu_{c}\left(\mu_{d}\right.$ and $\mu_{c}$ are the dynamic viscosities of the droplet and the carrier liquid respectively) and the Capillary number $C a=\mu_{c} R G / \sigma(R, G$, and $\sigma$ are the drop radius, local shear rate, and surface tension, respectively) which is the ratio of viscous to surface tension forces. In flows where the range of velocity gradients experienced by the dispersed 
phase is limited and deviation of the drop shape from sphericity is small, analytical solutions to compute the shape of the drop exist [60-62]. However, when the flow field becomes complex and deviation from sphericity is beyond a critical value, the constitutive equations become intractable.

In turbulent flows, when the dispersed phase is larger than the Kolmogorov length scale, inertial forces play a primary role in deforming the drops which may eventually lead to breakup. Kolmogorov [63] and Hinze [64] proposed one of the earliest models to study the process of deformation and breakup of such droplets dispersed into a turbulent carrier flow. The breakup of these droplets and its distribution in homogeneous isotropic turbulence was further investigated by Perlekar et al. [65] using a multicomponent lattice Boltzmann method. Through Lagrangian tracking of individual droplets, these simulations showed that the local Weber number at the droplet position emerges as a crucial parameter and is strongly correlated with the degree of deformation and thus eventual breakup. A series of studies used the front-tracking approach to investigate the influence of inertial size spherical and deformable bubbles on the wall drag in a turbulent channel flow [7, 12, 14, 15]. While the process of breakup and coalescence of the droplets cannot be modelled yet using this approach, it provides useful insight into the effect of deformability on the dynamics of bubbles much larger than the Kolmogorov scale $\left(d_{b} \sim 20 \eta_{k}\right)$ in wall bounded turbulence.

When the drops are smaller than Kolmogorov scale (sub-Kolmogorov), local viscous forces acting on the droplet become dominant in the deformation process thus making the capillary number $(\mathrm{Ca})$ relevant. In this situation Cristini et al. [66] used the boundary integral formulation to study the deformation and breakup process of drops dispersed into an isotropic turbulent flow with $R e_{\lambda} \sim 50$ ( $\lambda$ is the Taylor microscale). The process of drop deformation, elongation, neck formation and eventual breakup into satellite droplets was completely resolved and captured in these simulations. However, such fully resolved simulations come at an expense of the intensity of turbulence that can be achieved in the carrier flow field and also the number of droplets that can be simulated simultaneously.

Due to the huge computational costs involved, simulating millions of fully resolved droplets or bubbles which can deform, breakup and coalesce in highly turbulent flow fields is still beyond reach [7]. However, useful insights in such flows can still be obtained with the use of sub-grid models that can capture the motion and deformation of the dispersed phase. Instead of fully resolving the interaction between the dispersed phase and carrier phase, semi-empirical or phenomenological models can be used to track, deform, breakup, or coalesce a large number of droplets or bubbles in 
highly turbulent environments. Such an approach has been adopted by Biferale et al. [67], where they study the deformation and orientation statistics of approximately $10^{4}$ sub-Kolmogorov neutrally buoyant drops in homogeneous isotropic turbulence. The drops were assumed to be tri-axial ellipsoids and the shape and orientation of these passively advected drops was calculated using a phenomenological model proposed by Maffettone and Minale [68]. More recently, Babler et al. [69] studied the breakup of approximately $10^{5}$ colloidal aggregates in canonical flows like turbulent channel flow, developing boundary layer and homogeneous isotropic turbulence. A similar work by Marchioli et al. [70] investigated the statistics of ductile rupture of colloidal aggregates tracked as massless particles in a turbulent channel flow. A simple criteria for aggregate breakup or rupture was adopted by both Babler et al. [69] and Marchioli et al. [70] where the occurrence of a colloid breakup is directly related to the local hydrodynamic stress being higher than a critical threshold stress. In these studies, simulations of millions of droplets or colloidal aggregates in highly turbulent environments has been possible only due to the coupling of direct numerical simulations (DNS) of the carrier phase with a semi-empirical or phenomenological model for the deformation and breakup process. In this chapter we use a similar approach and couple a sub-grid deformation model along with DNS of the carrier flow to simultaneously track approximately $10^{5}$ neutrally buoyant sub-Kolmogorov deformable passive drops in a turbulent TC flow.

As mentioned previously, the driving of the TC system can induce several transitions in the flow from a purely laminar state to a fully turbulent system. Here, the outer cylinder is kept stationary $\left(R e_{o}=0\right)$ and only the inner cylinder is rotated until the system transitions into turbulence. We consider two different inner cylinder Reynolds numbers; one where there is a strong footprint of coherent structures i.e. the Taylor rolls and a higher $R e_{i}$ where the turbulence intensity is higher and the coherence in the rolls is relatively weaker. For each of these drivings, we vary the capillary number $(\mathrm{Ca})$ and viscosity ratio $(\hat{\mu})$ of the droplets with the carrier fluid. The drops are treated as passive inertia-less particles while the deformation and orientation of these drops is computed based on the phenomenological model proposed by Maffettone and Minale [68] (hereafter referred to as 'MM' model).

This approach is similar to the one adopted by Biferale et al. [67] for drops dispersed in homogeneous isotropic turbulence (HIT) and also by de Tullio et al. [71] to predict hemolysis by tracking several deformable red blood cells. A significant difference in this chapter in comparison to the work of Biferale et al. [67] is that we study the deformation of drops in a highly inhomogeneous and anisotropic carrier flow (here Taylor-Couette) as opposed to a homogeneous-isotropic turbulent 
environment. Given that TC flow is one of the paradigmatic systems in wall-bounded turbulent flows, we expect that the results from this study would have significant relevance in understanding deformation of drops in other canonical wall-bounded flows such as pipe flow, channel flows, convective turbulence etc. [72, 73]. Here, we consider only neutrally-buoyant sub-Kolmogorov drops which we model with tracer-like zero-way coupling. Although this restricts us from studying the physics behind turbulence modification it does allow us to study the deformation dynamics of drops in a inhomogeneous-anisotropic system. Additionally, in a later section we present arguments on why the tracer-like zero-way coupling is a reasonable approximation for sub-Kolmogorov neutrally buoyant drops. One open question is the spatial dependence of the degree of deformation of the dispersed phase and how the shapes of the drops vary as they are transported in the flow. Are the drops more prolate or more oblate like and does the capillary number and viscosity ratio have an influence on the shape? How do these drops deform in different regions of the flow like the Taylor rolls, plume ejection and plume impact regions, respectively? What is the preferential direction of orientation of these drops in the boundary layers and bulk region? These are some questions we attempt to answer in this chapter.

In the following section, we elaborate on the numerical schemes and methods employed to solve the equations of the carrier phase and the dispersed phase and also give a brief review on the MM model which is used to compute the deformation and orientation characteristics of the drops. In section 4.3 we discuss the results of our simulations where we analyse the averaged profiles of extent of deformation of the drops, probability distribution functions (PDF) of the extent of anisotropy in the dispersed phase and their orientational preferences for different $C a$ and $\hat{\mu}$.

\subsection{Governing Equations and Numerical Details}

For the carrier flow, DNS was used to solve the Navier-Stokes equations in cylindrical coordinates using a second-order accurate finite-difference scheme with fractional time-stepping $[30,31]$ as has been described in $\S 1$. The code for DNS along with two-way coupled Lagrangian tracking has also been validated and used previously for studying the effect of buoyant spherical bubbles on the net drag reduction in a turbulent Taylor-Couette flow (cf. §2) [74]. For the purpose of this study the radius ratio and aspect ratio of the TC setup is fixed to $\eta=r_{i} / r_{o}=0.833$ and $\Gamma=L / d=4$, respectively. Periodic boundary conditions are employed in the azimuthal and axial directions and a non-uniform grid spacing (clipped Chebychev clustering) is employed in the wall normal direction. Since we employ periodic boundary conditions in the 
axial direction, we have ensured through additional test simulations that the mean profiles and other relevant statistics discussed in section 4.3 are not influenced by increasing the aspect ratio $[74,75]$.

The dispersed phase is composed of neutrally buoyant sub-Kolmogorov drops which are treated as passive tracers [67]. The dispersed phase is advected based on the local velocity of the carrier phase at the exact location of each drop. Here it is important to note that the drops being treated as massless passive tracers with no back-reaction on the flow is a zero-way coupled approximation in comparison to the one-way/two-way/four-way coupled approaches typically adopted in particle laden turbulent flow studies [76]. Such an approximation simplifies the problem since computing drag, lift and added mass closures for continuously deforming drops is a highly non-trivial issue and is not the focus of this chapter. Nonetheless, given that the dispersed phase is neutrally buoyant and sub-Kolmogorov in size $\left(R / \eta_{k} \sim 0.025-0.075\right)$ even during deformation which results in extremely small particle time scales (or in other words low Stokes numbers) and minimal wake effects, it can be argued that the behaviour of these drops is close to that of passive tracers.

Since the dispersed phase is treated in a Lagrangian manner, the position of each droplet does not necessarily coincide with the location of grid nodes on the carrier phase mesh. This limitation is overcome with the use of a tri-linear interpolation scheme to compute the velocity of the carrier phase at the exact droplet position. The droplet position is updated after every time step according to equation $3.1 \mathrm{using}$ a second order Runge-Kutta scheme ( $\mathbf{v}$ is the interpolated carrier phase velocity at the droplet position and $\mathbf{x}_{p}$ is the droplet position vector).

$$
\frac{d \mathbf{x}_{p}}{d t}=\mathbf{v}
$$

In order to couple the tracking of the droplets with deformation, the droplets are assumed to be tri-axial ellipsoids. Under this assumption, the drop shape and orientation can be easily described in the form of a second-order positive-definite symmetric tensor $\boldsymbol{S}$ (shape tensor). This tensor satisfies the condition $\boldsymbol{S}^{-1}: \mathbf{x x}=1$, where $\mathbf{x}$ is the position vector of any point on the ellipsoid surface relative to its origin. For any known tensor $\boldsymbol{S}$, the eigenvalues of the shape tensor give the square of semi-axes of the ellipsoid while the eigenvectors give the orientation of the semi-axes of the ellipsoid. In case of ellipsoidal drops, the MM model proposes a phenomenological evolution equation of the shape tensor $S$ based on the competing actions of drag which tends to elongate the droplet and surface tension which tends to recover the spherical shape corresponding to minimum surface energy. In dimensional form this equation reads: 


$$
\frac{d \boldsymbol{S}}{d t}-(\boldsymbol{\Omega} \cdot \boldsymbol{S}-\boldsymbol{S} \cdot \boldsymbol{\Omega})=-\frac{f_{1}}{\tau}(\boldsymbol{S}-g(\boldsymbol{S}) \boldsymbol{I})+f_{2}(\boldsymbol{E} \cdot \boldsymbol{S}+\boldsymbol{S} \cdot \boldsymbol{E}) .
$$

We now non-dimensionalise as follows: $S^{*}=S / R^{2}$ where $R$ is the radius of the undistorted spherical drop. $\boldsymbol{E}^{*}=\boldsymbol{E} / G$ and $\boldsymbol{\Omega}^{*}=\boldsymbol{\Omega} / G$ are the non-dimensional strain rate and vorticity rate tensors; $\boldsymbol{E}=0.5\left[\nabla \boldsymbol{u}+\nabla \boldsymbol{u}^{T}\right], \boldsymbol{\Omega}=0.5\left[\nabla \boldsymbol{u}-\nabla \boldsymbol{u}^{T}\right]$, and $G=1 / \tau_{\eta}$ is the inverse Kolmogorov turbulent time scale. To compute $\tau_{\eta}$, we use the exact relations between the driving torque and the volume averaged dissipation in the flow as given in Eckhardt et al. [36]. The capillary number $C a$ which is the ratio of the viscous forces to the interfacial forces and can also be defined as the ratio of the interfacial relaxation time $\left(\tau=\mu_{c} R / \sigma\right)$ to the characteristic flow time $\left(\tau_{\eta}\right)$ i.e. $C a=\tau / \tau_{\eta} ; \mu_{c}$ is viscosity of the carrier phase and $\sigma$ the interfacial tension. Since the maximum Capillary number $C a$ we consider in this study is $C a=0.3$ i.e $\tau<\tau_{\eta}$, the deformation dynamics are inherently decoupled from the fluctuations in the underlying turbulent flow.

In non-dimensional form the evolution equation for the shape tensor $\left(\boldsymbol{S}^{*}\right)$ can be written as follows:

$$
\frac{d \boldsymbol{S}^{*}}{d t}-C a\left(\boldsymbol{\Omega}^{*} \cdot \boldsymbol{S}^{*}-\boldsymbol{S}^{*} \cdot \boldsymbol{\Omega}^{*}\right)=-f_{1}\left(\boldsymbol{S}^{*}-g\left(\boldsymbol{S}^{*}\right) \boldsymbol{I}\right)+f_{2} C a\left(\boldsymbol{E}^{*} \cdot \boldsymbol{S}^{*}+\boldsymbol{S}^{*} \cdot \boldsymbol{E}^{*}\right) .
$$

Since we consider both fluids to be incompressible and no occurrence of droplet breakup or coalescence, the volume of each droplet must be conserved. This implies that the third invariant or the determinant of the tensor $\boldsymbol{S}$ must be constant and the function $g\left(\boldsymbol{S}^{*}\right)=3 I I I_{s} / I I_{s}$ embedded into the evolution equation ensures volume conservation $\left(I I_{s}\right.$ and $I I I_{s}$ are the second and third invariant of the shape tensor, respectively). The parameters $f_{1}$ and $f_{2}$ depend only on the viscosity ratio $(\hat{\mu})$ and are chosen to make the MM model recover the asymptotic limits for small $\mathrm{Ca}$, viscosity ratio $1 / \hat{\mu} \rightarrow 1$ and $\hat{\mu}=1$ and is given by Maffettone and Minale [68] as follows:

$$
f_{1}=\frac{40(\hat{\mu}+1)}{(2 \hat{\mu}+3)(19 \hat{\mu}+16)} \quad f_{2}=\frac{5}{2 \hat{\mu}+3},
$$

Here we briefly discuss the various factors affecting the choice of the functions $f_{1}$ and $f_{2}$. The expression for $f_{2}$ in equation (5.18) under-predicts the net deformation when $C a$ and $\hat{\mu}$ are large and this can be corrected by including an additional dependence of $f_{2}$ on $\mathrm{Ca}[67,68]$. In particular, this correction becomes significant when the droplet Capillary number is relatively high i.e $\mathrm{Ca}>0.3$ which is the upper limit of the $\mathrm{Ca}$ chosen in our study [68]. Additionally, when the droplet is 
relatively large in comparison to the gap between the shearing walls i.e $R / h>0.15$ ( $h$ is the confinement length scale) confinement effects become important and the functions $f_{1}$ and $f_{2}$ also depend on $R / h$ [77-79]. In the current study we consider only sub-Kolmogorov drops such that they see locally a linearised flow field and thus the ratio $R / h$ (where the confinement length scale is based on the cylinder gap-width) is very small i.e. $R / h \sim 0.01$ in comparison to the threshold value of 0.15 . When the drops approach the walls, the boundary layer thickness can be taken to be the confinement length scale and since we only consider sub-Kolmogorov droplets the maximum value of the confinement ratio $R / h$ based on the boundary layer thickness is 0.1 . Based on the above arguments, the simplified forms of the functions $f_{1}$ and $f_{2}$ given in equation (5.18) are chosen for this study.

The constitutive equation for the evolution of the shape tensor (equation 3.3) has terms which would require the evaluation of the local strain rate and rotation rate tensor at the location of the droplet. An interpolation scheme similar to the one used for computing the velocity in equation (3.1) is implemented for this purpose. Analogous to the carrier phase, azimuthal and axial periodicity is adopted for the dispersed phase, i.e. when the droplets exit an azimuthal or axial boundary they are re-entered at the opposite boundary with the same velocity, shape and orientation. An ad-hoc approach needs to be implemented for the interaction of a droplet with the wall. Although the droplets which are treated as passive tracers never cross the wall boundaries since the drops are tracked based on the local fluid velocity at its centre of mass, the edge of a highly deformed droplet near a wall can cross the wall-boundary leading to an unphysical situation. To overcome this we construct a hypothetical bounding box around a deformed droplet with the centre of mass of this box coinciding with the centre of mass of the droplet. The boundaries of this box are then used as the reference planes for modelling the elastic collision. Here it is important to note that modelling the collision between a continuously deforming droplet and a wall is an extremely challenging and interesting subject in itself. The influence of our collision approximation on the deformation statistics is discussed in detail in the next section.

The entire simulation for a single case consists of two parts. Firstly, the carrier phase is simulated without any dispersed phase until it reaches a statistically stationary state. At this point the simulation is stopped and $10^{5}$ sub-Kolmogorov spherical drops are introduced in the gap width at random positions and the simulation is allowed to continue. The trajectories and the information on the shape tensor of every droplet is recorded at a frequency of approximately 20 snapshots every $\tau_{\eta}$ for almost 100 full inner cylinder rotations. The complete simulation set consists 


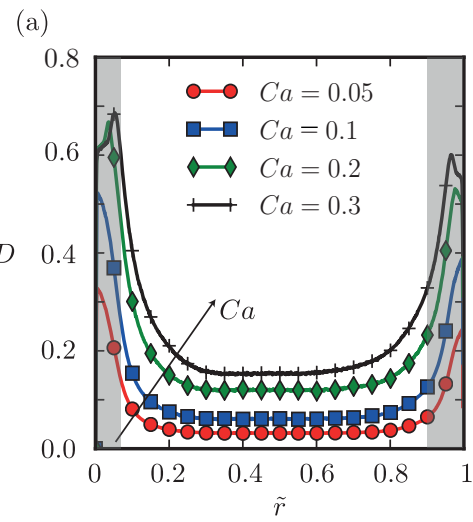

(b)

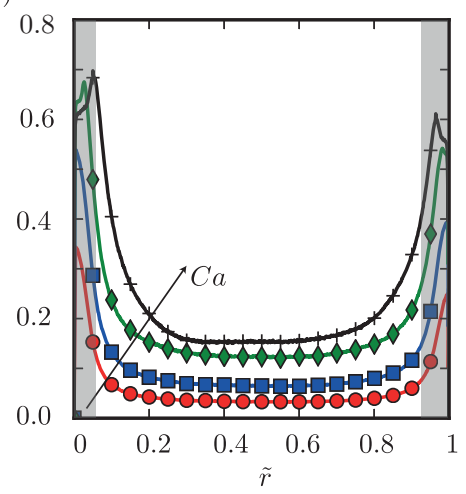

Figure 3.1: Azimuthally, axially and time averaged radial profiles of deformation parameters $(D)$ for four different $C a$; (a) $R e_{i}=2500$ (b) $R e_{i}=5000$. The viscosity ratio $\mu=1$. The direction of the arrow indicates increasing $C a$. The grey shaded areas near the left and right extremes of the $\tilde{r}$ axis represent the inner and outer cylinder boundary layer thickness, respectively.

of two different inner cylinder Reynolds numbers $\left(R e_{i}=2500,5000\right)$, four different Capillary numbers $(\mathrm{Ca}=0.05,0.1,0.2$ and 0.3$)$ and two different viscosity ratios $(\hat{\mu}=1$ and 100).

\subsection{Results}

We first analyse the statistics of the shape and orientation of the droplets for different Reynolds $R e_{i}$ and capillary numbers $C a$ while keeping the viscosity ratio fixed at $\hat{\mu}=1$. Since we consider the droplets to be tri-axial ellipsoidal, we denote the lengths of the three semi-axes by $d_{1}, d_{2}$ and $d_{3}\left(d_{1}<d_{2}<d_{3}\right)$; i.e. $d_{1}$ and $d_{3}$ are the lengths of the minor and major semi-axes, respectively.

\subsubsection{Droplet shape distribution}

In order to quantify the degree of deformation in the droplet shape, we make use of a non-dimensional deformation parameter defined as $D=\left(d_{3}-d_{1}\right) /\left(d_{3}+d_{1}\right)$ $[58,59,67,68,80,81]$. When $D \sim 0$, the droplet is close to spherical, while $D \gg 0$ implies the droplet is highly deformed. In order to properly quantify the correlation between the spatial position of the droplets and the degree of deformation, we plot the radial profiles of azimuthally, axially and time averaged deformation parameter 

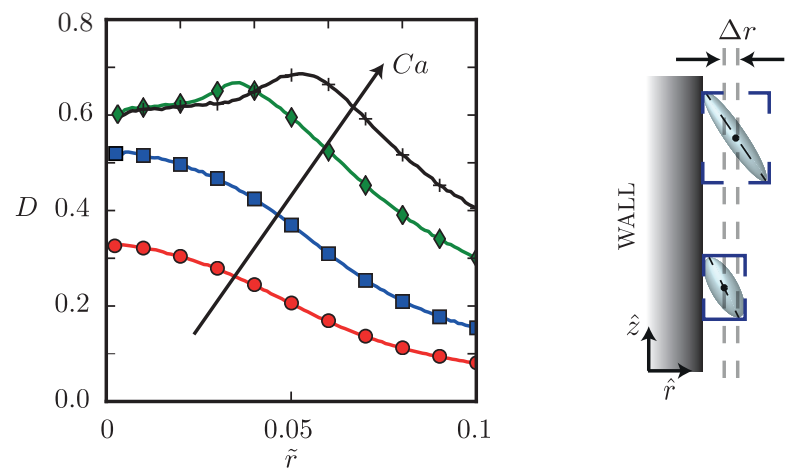

Figure 3.2: Zoomed in plot of figure 3.1a. The schematic on the right shows interaction of a highly deformed drop (top) and a relatively less deformed drop (bottom) with the wall. The dotted line around each droplet shows the hypothetical bounding box constructed around each droplet. The centre of mass of a less deformed drop can approach more closer to the wall as compared to a highly deformed drop.

for droplets with four different $C a$ in figure 3.1. The radial position of the drops are normalised using $\tilde{r}=\left(r-r_{i}\right) /\left(r_{o}-r_{i}\right)$; the drop is close to the inner cylinder when $\tilde{r} \sim 0$ and close to the outer cylinder when $\tilde{r} \sim 1$. Along with the averaged profiles we show the inner boundary layer (IBL) and outer boundary layer (OBL) thickness using grey shaded areas which have been computed following the approach described in previous works $[53,55,74]$.

We can clearly observe in figure 3.1 that the capillary number $C a$ has a strong influence on the mean deformation parameter $D$. As expected, $D$ increases with increasing $\mathrm{Ca}$ due to weakening surface tension forces. Additionally, as noted from figure 3.1 a clear indication of the spatial dependence of the deformation parameter $D$ is observed. $D$ is higher near the boundary layers (marked by grey shaded areas) for both Reynolds numbers as compared to the bulk where it is almost three times lower. Additionally, we also find that when $C a=0.2$ and $C a=0.3$, the peak of the deformation parameter $D$ lies away from the inner cylinder. To observe this deviation more clearly, in figure 3.2 we show a zoomed-in plot of figure 3.1(a) and it can be clearly seen that for $C a=0.2$ and $C a=0.3$ there is a slight increase in $D$ away from the wall and the occurrence of this peak moves away from the wall with increasing $C a$. This is just a consequence of the modelling of interaction of the droplet with the wall. Since we assume elastic collision between the bounding box of a deformed droplet and the wall, the centre of mass of a highly deformed droplet lies further away from the 
wall as compared to a less deformed droplet as shown in the schematic of figure 3.2. Indeed, this also depends on the relative orientation of the droplets with the wall and we will analyse this in detail in later sections. Through several test simulations it has been ensured that the shift in peak of $D$ is indeed due to the collision and not due to any additional boundary layer dynamics (e.g. droplets modelled without any collision do not show any shift in the peak of $D$ ). This argument is further strengthened in section 3.3 where we study the deformation of more viscous drops.

Similar to figure 3.1 we plot axial profiles of the azimuthally, radially and time averaged deformation parameter $D$ as a function of the normalised axial position $\tilde{z}=z / d$ for $R e_{i}=2500$ and 5000 and for different $C a$ in figure 3.3. When $R e_{i}=2500$ (c.f. bottom panel of figure 3.3a), we observe that regardless of the capillary number, $D$ is higher near the plume ejection regions of the inner cylinder i.e $\tilde{z} \sim 1$ and 3 (also refer to the central and top panel in figure 3.3(a) which show an instantaneous snapshot of the carrier phase azimuthal velocity along with a quiver plot of a zoomed in section). This is a result of relatively high strain rates stretching the ellipsoidal drops in the plume ejection region near the inner cylinder; an effect likely to be produced by the Taylor rolls which make locally thinner or thicker the boundary layer produced by the cylinder rotation. A similar observation is made even for $R e_{i}=5000$ in figure 3.3(b) where the plume ejection regions near the inner cylinder are at approximately $\tilde{z}=$ 1.75 and 3.75. Although relatively high strain rates are observed in the plume ejection regions, the plumes themselves which extend from the inner cylinder into the bulk have a relatively lower strain rate owing to a strong azimuthal velocity component. This causes a sudden drop in $D$ at the exact axial position where the plumes extend into the bulk, an effect which is seen very clearly for $R e_{i}=2500$ due to the more coherent nature of the plumes (figure 3.3(a)). However, when the Reynolds number is increased the plumes are more erratic and turbulent and the sudden drop in $D$ gets averaged out and is not observed any more. The differences in plume structure in the bulk of the flow for both Reynolds number is clearly visible in the instantaneous snapshots of the azimuthal velocity in figure 3.3 .

Deformation is directly related to the strain-rates experienced by the droplets. In order to further understand the correlation between the strain rates in the flow and the net deformation observed we plot the averaged profiles of normalised viscous dissipation (square of the magnitude of the strain rate) against the radial and axial position. The total dissipation per unit mass is computed as a volume and time average of the local dissipation rate [36]. After spatial averaging the net dissipation is normalised using its corresponding maximum value $\hat{\epsilon}=\epsilon / \max (\epsilon)$ and is plotted against the normalised radial position in figure $3.4(\mathrm{a})$ and the normalised axial 

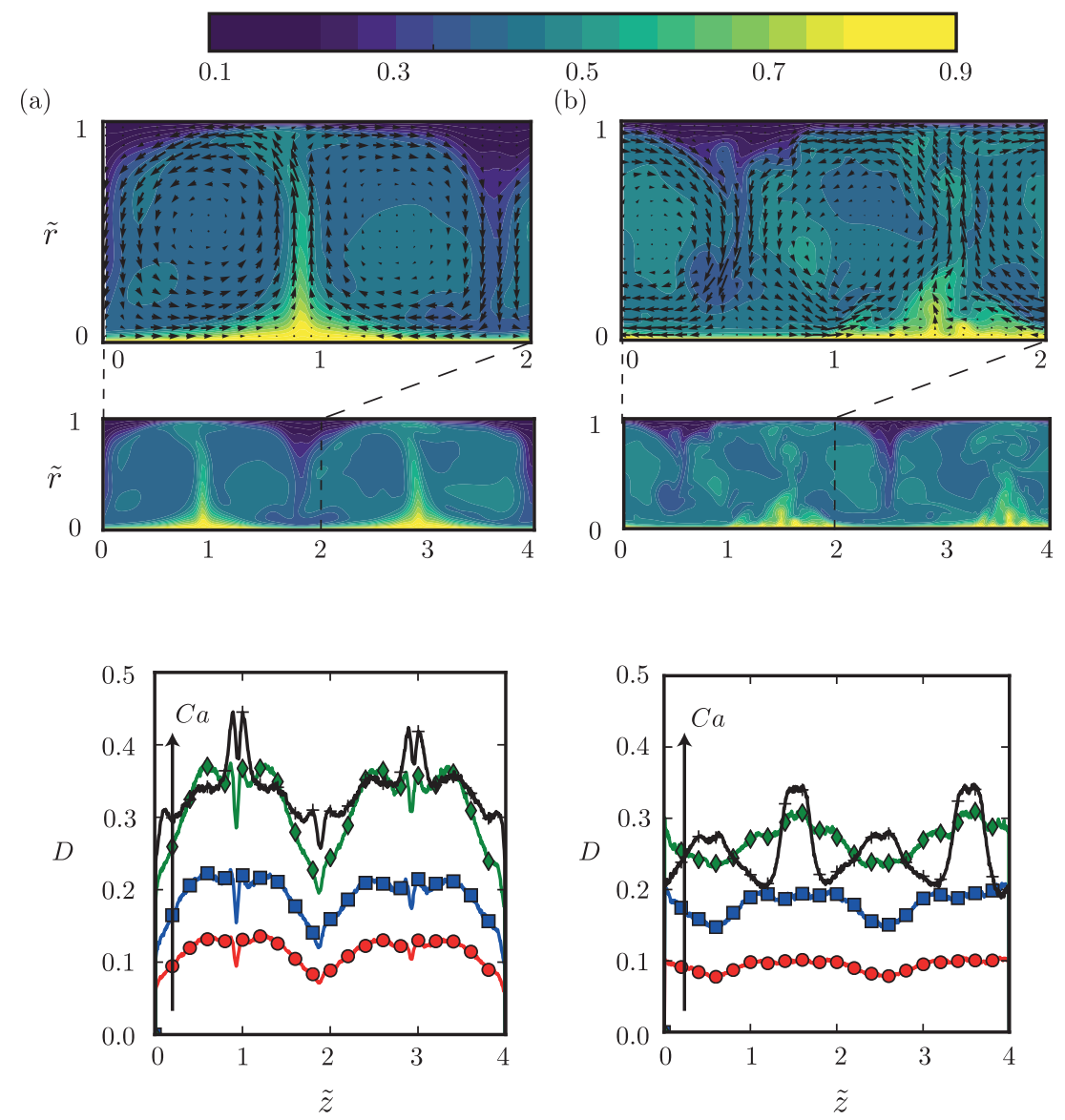

Figure 3.3: Azimuthally, radially and time averaged axial profiles of the deformation parameter $(D)$ for four different $C a$; (a) $R e_{i}=2500$ (b) $R e_{i}=5000$. The viscosity ratio $\mu=1$. The direction of the arrow indicates increasing $\mathrm{Ca}$ (refer to figure 3.1 for description of the markers). Instantaneous snapshots of the azimuthal velocity of the corresponding $R e_{i}$ are shown on the top of each plot to better visualise the plume ejections and the Taylor rolls along with a quiver plot of half the domain to show the direction of flow. The colour scale shows the instantaneous azimuthal velocity. 
(a)

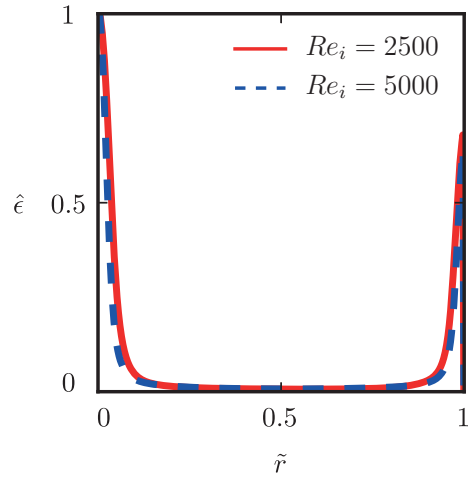

(b)

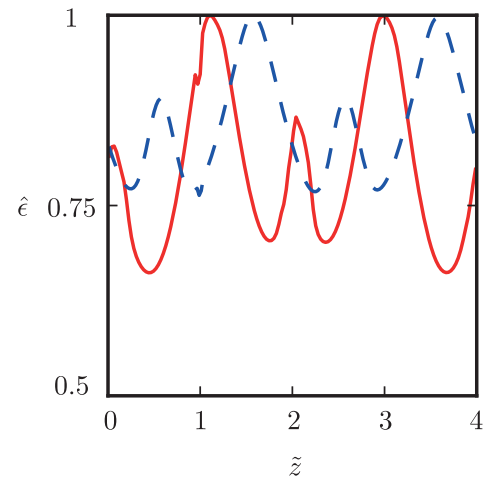

Figure 3.4: Azimuthally and time averaged profiles of the viscous dissipation (a) radial profiles (b) axial profiles. Net dissipation is computed as a volume and time average of local dissipation rate and is normalised using its corresponding maximum value $\hat{\epsilon}=\epsilon / \max (\epsilon)$.

position in figure 3.4(b). On comparing the averaged radial and axial profiles of the deformation parameter (figures 3.1 and 3.3) along with the normalised dissipation (figures 3.4(a) and (b)) a very strong correlation is observed between both quantities, thus confirming the hypothesis that high local strain rates are crucial for strong drop deformation.

Until now we have looked at the variation of the deformation parameter $D$ with the radial and axial distance in the TC domain. Although $D$ can be used to study whether the droplet is close to spherical or highly deformed, it cannot be used to distinguish between oblate (disk-like) and prolate (cigar-like) droplets. In order to make this distinction, Biferale et al. [67] used the $s^{*}$ parameter which was introduced by Lund et al. [82] to quantify the probability distribution of strain rate tensor eigen-values in turbulent flows. The shapes of the droplets can also be categorised based on simple ratios of the semi-axes i.e. $d_{2} / d_{1}$ and $d_{3} / d_{1}$ (where $d_{1}<d_{2}<d_{3}$ ). When the droplet is close to spherical $d_{2} / d_{1} \sim d_{3} / d_{1} \sim 1$, while for deformed droplets both $d_{2} / d_{1}>1$ and $d_{3} / d_{1}>1$. When the droplets are deformed they can additionally be categorised into oblate (disk-like) ellipsoids when $d_{2} / d_{1} \sim d_{3} / d_{1}$ and prolate (cigar-like) ellipsoids when $d_{2} / d_{1} \ll d_{3} / d_{1}$.

In figure 3.5, we plot the probability distribution functions (PDF's) of the ratio of semi-axes $\left(d_{2} / d_{1}\right.$ and $\left.d_{3} / d_{1}\right)$ of droplets for two different capillary numbers $C a=0.05$ and 0.2 while $R e_{i}=2500$ and $\hat{\mu}=1$. The three different panels for each capillary number correspond to the position of the droplet in the inner boundary layer (IBL), 

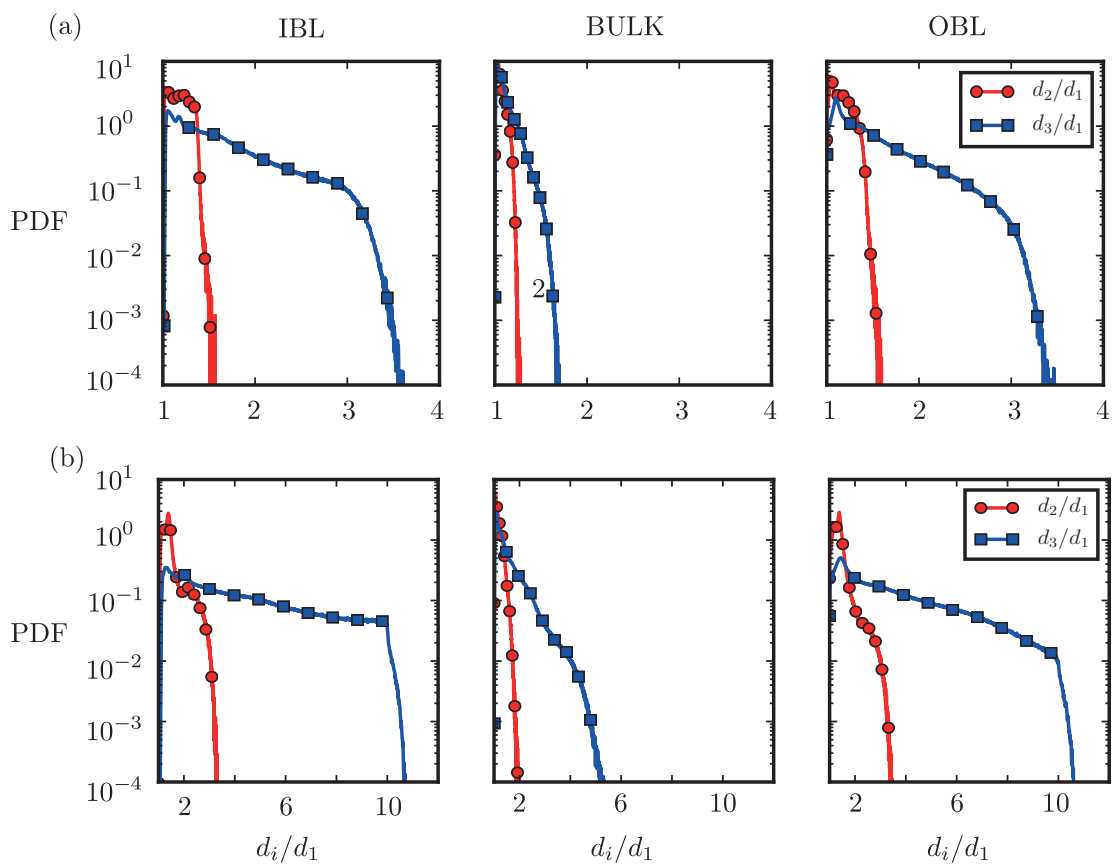

Figure 3.5: Probability distribution function (PDF) of the ratio of semi-axes $\left(d_{1}<d_{2}<d_{3}\right)$ for $R e_{i}=2500$ and $\hat{\mu}=1$ (a) $C a=0.05$ (b) $C a=0.2$. The three panels for each $C a$ correspond to droplets in the inner boundary layer (IBL), bulk and outer boundary layer region (OBL). 
bulk region (BULK), and the outer boundary layer (OBL). When $C a=0.05$, the droplets are almost spheroidal or in other words axis-symmetric (i.e. $d_{2} / d_{1} \sim 1$ ). In the bulk where the strain rates are relatively weaker as compared to the boundary layer region, the droplet is close to being spherical with minimum deformation (i.e. $\left.d_{2} / d_{1} \sim d_{3} / d_{1} \sim 1\right)$. Additionally, the PDF's of $d_{3} / d_{1}$ in the IBL and OBL have much wider tails indicating that the droplets are more prolate in comparison to the bulk region. Biferale et al. [67] found that with increasing capillary number ellipsoidal drops in homogeneous isotropic turbulence tend to be more cigar-like or prolate where the major axis $\left(d_{3}\right)$ is relatively much larger than the other two axes $\left(d_{1}, d_{2}\right)$. A similar behaviour can be observed in the bottom panels of figure 3.5 when the capillary number is increased to $C a=0.2$. The tails of $d_{3} / d_{1}$ become much wider in the IBL and OBL regions as compared to $C a=0.05$ which is a consequence of the weaker surface tension forces for higher capillary number drops. An additional consequence is that the droplets now tend to become less spheroidal and more tri-axial.

\subsubsection{Orientational behaviour of the drops}

In addition to the shape and size of the ellipsoidal droplets it would also be interesting to understand their orientational preference. A number of studies [83-85] investigating the orientation behaviour of axis-symmetric solid ellipsoidal particles in wall bounded turbulent flows found that the major axis of the particles tend to align with the stream-wise direction near the wall. Rigid rod/fibre like particles immersed in a isotropic turbulent flow align with the vorticity and the strain rate eigen-vectors and their orientational behaviour has a strong dependence on the shape [86-88]. The shape and size of solid ellipsoidal particles does not depend on their spatial position and underlying flow conditions. However, for deforming droplets the orientation dynamics can be strongly coupled to the shape evolution which would in turn depend on the local flow conditions. Biferale et al. [67] found that neutrally buoyant ellipsoidal droplets dispersed into a homogeneous isotropic turbulent flow aligned with the most extensional strain-rate eigen direction and the vorticity. With an increase in the capillary number $\mathrm{Ca}$ the alignment is lost due to an increase in the interfacial relaxation time.

Here we describe the relative orientation of the major axis of the ellipsoidal droplets with respect to the coordinate system in the form of direction cosines of the angle between them i.e. $l=\cos \theta_{\hat{\theta}}, m=\cos \theta_{\hat{r}}, n=\cos \theta_{\hat{z}}$, where $l, m, n$ are the direction cosine of the orientation with $\hat{\theta}$ (stream-wise), $\hat{r}$ (wall-normal), and $\hat{z}$ (span-wise) directions, respectively. In order to study the statistics of droplet orientation in detail, we plot the PDF's of the cosine of the angle between the droplet major axis and the 

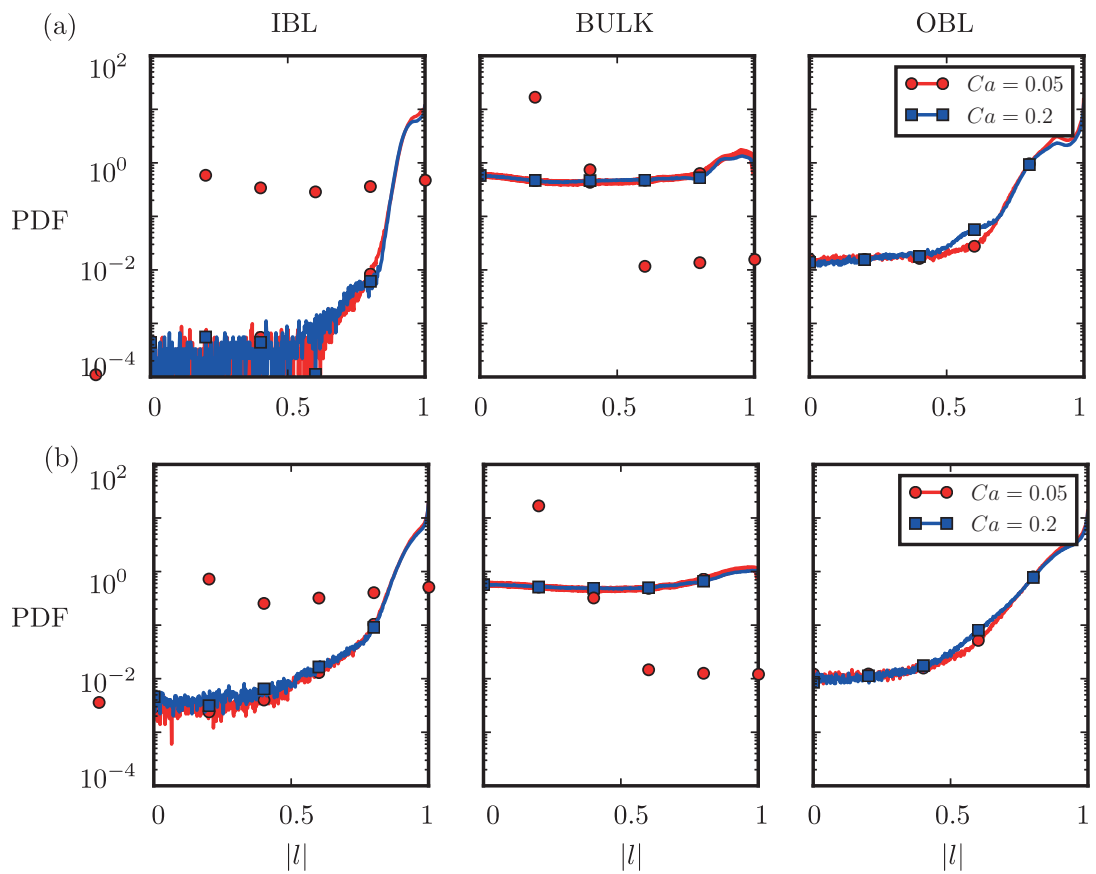

Figure 3.6: Probability distribution functions of the stream-wise orientation of the major axis of droplets for $C a=0.05$ and $C a=0.2$ (a) $R e_{i}=2500$ (b) $R e_{i}=5000$. The three panels for each $R e_{i}$ correspond to droplets in the inner boundary layer (IBL), bulk and outer boundary layer region (OBL). 
(a)
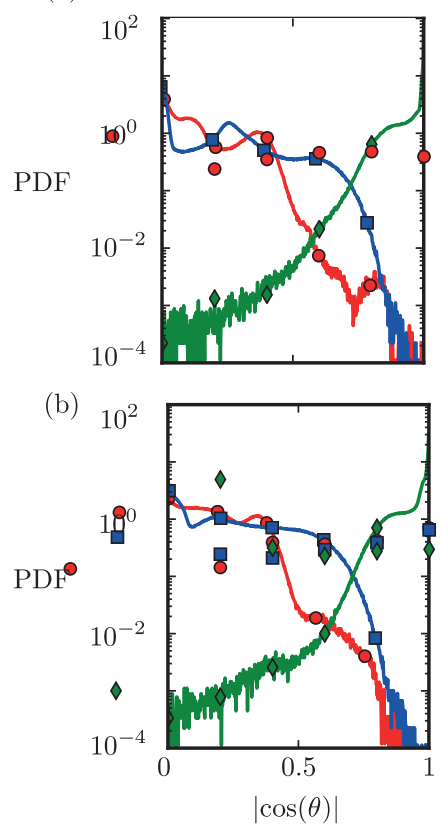

BULK
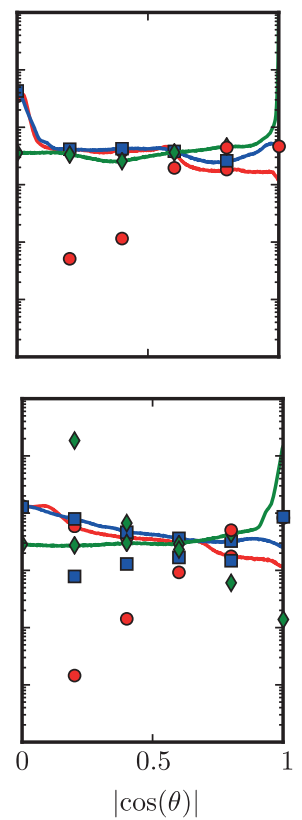

OBL
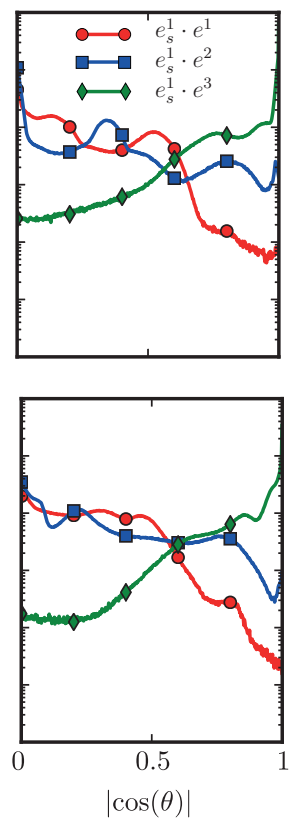

Figure 3.7: Probability distribution functions of the cosine of the angle between the eigen-vector of the major axis $\left(\mathbf{e}^{\mathbf{3}}\right)$ of the ellipsoid and three strain-rate eigen-directions $\left(\mathbf{e}_{\mathbf{s}}^{\mathbf{1}}, \mathbf{e}_{\mathbf{s}}^{\mathbf{2}}, \mathbf{e}_{\mathbf{s}}^{\mathbf{3}}\right) R_{i}=2500$ $\hat{\mu}=1$ for (a) $C a=0.05$ and (b) $C a=0.2$. 

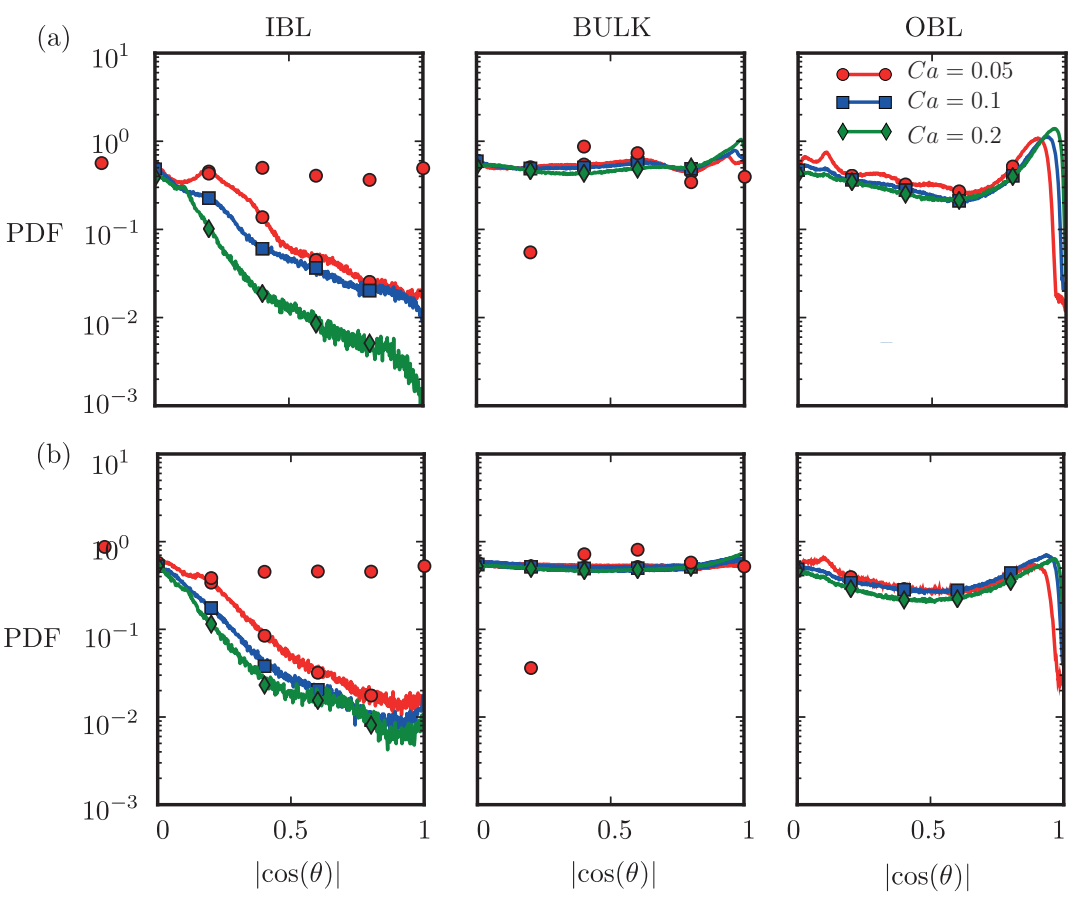

Figure 3.8: Probability distribution functions of the cosine of the angle between the eigen-vector of the semi-major axis $\left(\mathbf{e}^{\mathbf{3}}\right)$ of the ellipsoid and the vorticity $(\boldsymbol{\Omega}) \hat{\mu}=1$ for (a) $\operatorname{Re}_{i}=2500$ and (b) $R e_{i}=5000$. 
stream-wise direction in figure 3.6. We observe that the droplets tend to be strongly aligned with the stream-wise direction (i.e. $l \sim 1.0$ ) in the IBL and OBL. As can be seen from the left-most panels of figure 3.6, the alignment is more pronounced in the case of $R e_{i}=2500$ as compared to $R e_{i}=5000$. This may be possible due to the more erratic and time dependent nature of the boundary layer region and plume ejections from the inner cylinder in the case of $R e_{i}=5000$ as was also seen in the contour plots of figure 3.3(b). A similar behaviour is also seen in the case of solid ellipsoidal particles in the vicinity of a wall in a turbulent channel flow [83-85]. The capillary number $C a$ seems to have minimal influence on the orientational behaviour of drops suggesting that it is only dominant in the deformation process and not in the effective orientation of the drops. Since the drops are sub-Kolmogorov the drops experience uniform local flow conditions or in other words sample only the linear velocity gradients and thus the $C a$ number plays a important role only in deforming the droplet but not in its relative orientation especially given the fact that $C a$ is computed based solely on the ratios of the stretching forces and the retracting surface tension forces. Thus, no matter how much the droplet deforms the major axis is always oriented with the stream-wise direction in the boundary layers due to the strong deformation of the underlying fluid material element in the boundary layers. In the bulk, the orientation of the droplets is highly isotropic showing no specific preference in alignment with the stream-wise direction which is a consequence of the efficient mixing provided by the Taylor rolls.

Now we look at the orientation statistics of the major axis with respect to the local strain-rate eigen directions and vorticity. In figure 3.7 and figure 3.8 we plot the PDF's of the cosine of the angle between the eigen-vector of the major axis $\left(\mathbf{e}^{\mathbf{3}}\right)$, the three strain-rate eigen directions $\left(\mathbf{e}_{\mathbf{s}}^{\mathbf{1}}, \mathbf{e}_{\mathbf{s}}^{\mathbf{2}}, \mathbf{e}_{\mathbf{s}}^{\mathbf{3}}\right.$, where $\mathbf{e}_{\mathbf{s}}^{\mathbf{1}}$ and $\mathbf{e}_{\mathbf{s}}^{\mathbf{3}}$ are the compressive and extensional strain-rates, respectively) and vorticity $(\boldsymbol{\omega})$, respectively. In homogeneous isotropic turbulence, Biferale et al. [67] found that the major axis of neutrally buoyant ellipsoidal droplets align with both the most extensional strain-rate eigen direction and vorticity. In figures 3.7 and 3.8 we find that the preference of alignment with the strain rate eigen directions and vorticity depends again strongly on its relative spatial location.

In figure 3.7, we consider two different configurations with capillary numbers $C a=$ 0.05 and $C a=0.2$ for a fixed Reynolds number $R e_{i}=2500$. In the boundary layer regions the ellipsoid major axis has a strong preference to align with the extensional strain-rate eigen direction (here the extensional strain-rate direction in the boundary layers is the azimuthal direction). In the bulk, the preference is relatively more uniform while some traces of droplets aligning with $\mathbf{e}_{\mathbf{s}}^{\mathbf{3}}$ can still be found, an indication 


\section{DEFORMATION AND ORIENTATION STATISTICS OF SUB-KOLMOGOROV DROPS}

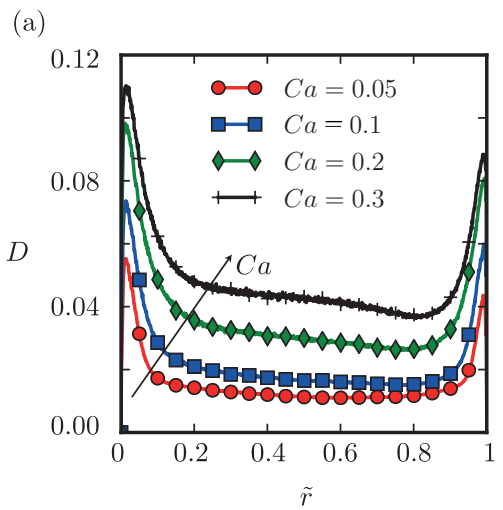

(b)

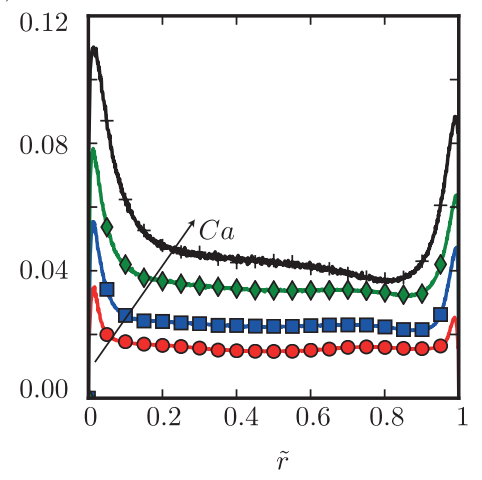

Figure 3.9: Azimuthally, axially and time averaged radial profiles of deformation parameters $(D)$ for four different $C a$; (a) $R e_{i}=2500$ (b) $R e_{i}=5000$. The viscosity ratio is $\hat{\mu}=100$. The direction of the arrow indicates increasing $C a$. The grey shaded areas near the left and right extremes of the x-axis represent the inner and outer cylinder boundary layer thickness, respectively.

that there seems to be a competition between the extensional strain-rates trying to align itself with the droplet major axis and the homogenising nature of the Taylor rolls. This effect is clearly seen when $C a=0.2$ given the prolate nature of the droplets (cf. figure 3.5). While the ellipsoidal droplets tend to align with the most extensional strain-rate direction in the inner boundary layer (IBL) region (left panels of figure 3.7) they prefer to be aligned orthogonally with the vorticity (left panel in figure 3.8). This is different from the behaviour of solid axis-symmetric ellipsoidal particles in an isotropic turbulent flow where rod-like particles tend to align with the vorticity $[87,89,90]$. The difference in behaviour is a consequence of the inhomogeneity and anisotropy of the carrier flow. Near the boundary layer, the vorticity is along the axial direction while the drops tend to align in the stream-wise direction which leads to the alignment preference seen in the left panel of figure 3.8. With increase in Reynolds number $R e_{i}$, the preference of alignment with the vorticity stays qualitatively similar. Overall, we find that the spatial position of the droplet is crucial in determining the orientational preference of the immersed droplets owing to the strong inhomogeneity and anisotropy in the field.

Until now we have looked at the effect of capillary number $C a$ and Reynolds number $R e_{i}$ on the droplet deformation and orientation statistics and in the next subsection we will study the effect of viscosity ratio $\hat{\mu}$. 


\subsubsection{Effect of viscosity ratio}

In figure 3.9 we plot the averaged radial profiles of the deformation parameter $D$ for different $C a$ in a high viscosity ratio system $\hat{\mu}=100$. On comparison with $\hat{\mu}=1$ (cf. figure 3.1) the degree of deformation is at least 5 times smaller for all capillary numbers. This behaviour can be intuitively expected as for the same strain rates the stretching is lower for more viscous drops given the additional viscous dissipation due to the internal flow in the drops. Additionally, due to the relatively smaller deformations the peaks in the deformation parameter $D$ lie close to the wall for all $\mathrm{Ca}$. While it is clear from figure 3.9 that there is a reduction in the overall deformation of the droplets, it would be interesting to look at the shape of the droplets as we did in figure 3.5 ; i.e. in the case of $\hat{\mu}=1$ the droplets were more prolate (cigar-like). In figure 3.10 we plot the PDF's of the ratio of semi-axes in the IBL, bulk region and OBL (similar to figure 3.5). It can be seen that the semi-axes ratios $d_{2} / d_{1} \sim d_{3} / d_{1}>1$ which is a clear indication that the droplets tend to be more oblate (disk-like) when $\hat{\mu}=100$. A similar behaviour is observed when the capillary number of the drops is increased. While we found previously that the capillary number plays an important role in the degree of deformation of the droplets, here we find that the droplet shape also depends strongly on the the viscosity ratio $\hat{\mu}$.

In order to understand these dependencies we need to go back to the equation governing the droplet shape (i.e. equation 3.3). By changing the viscosity ratio we influence the values of the parameters $f_{1}$ and $f_{2}$ which are calculated according to equation (5.18). $f_{1}$ and $f_{2}$ are monotonically decreasing functions of $\hat{\mu}$ and while the ratio $f_{1} / f_{2}$ is almost constant (see also figure 11 of [67]), $f_{1}$ and $f_{2}$ decrease by up to a factor of almost 50 by increasing the viscosity ratio from $\hat{\mu}=1$ to $\hat{\mu}=100$. This has a strong influence on the effective strengths of droplet stretching, relaxation, and rotation of the ellipsoidal droplets. More importantly, in comparison to low-viscosity ratio systems the influence of rotation becomes more dominant when the viscosity ratio is high [67]. Additionally, reducing $f_{1}$ results in an increase in the effective interfacial relaxation time (i.e. $\tau / f_{1}$ ) or in other words the surface tension force acting on the ellipsoidal droplet has lesser time to adjust to the local flow conditions resulting in some highly stretched drops. The increased influence of rotation can also result in the loss of a preferential stretching axis for the ellipsoid thus resulting in more oblate or disk-like droplets.

Here it is important to note that the decorrelating effect of straining with rotation has been observed previously in multiple studies. Girimaji et al. [91] studied the evolution of infinitesimal material elements in homogeneous isotropic turbulence using DNS where they concluded that the material line elements align poorly with the 

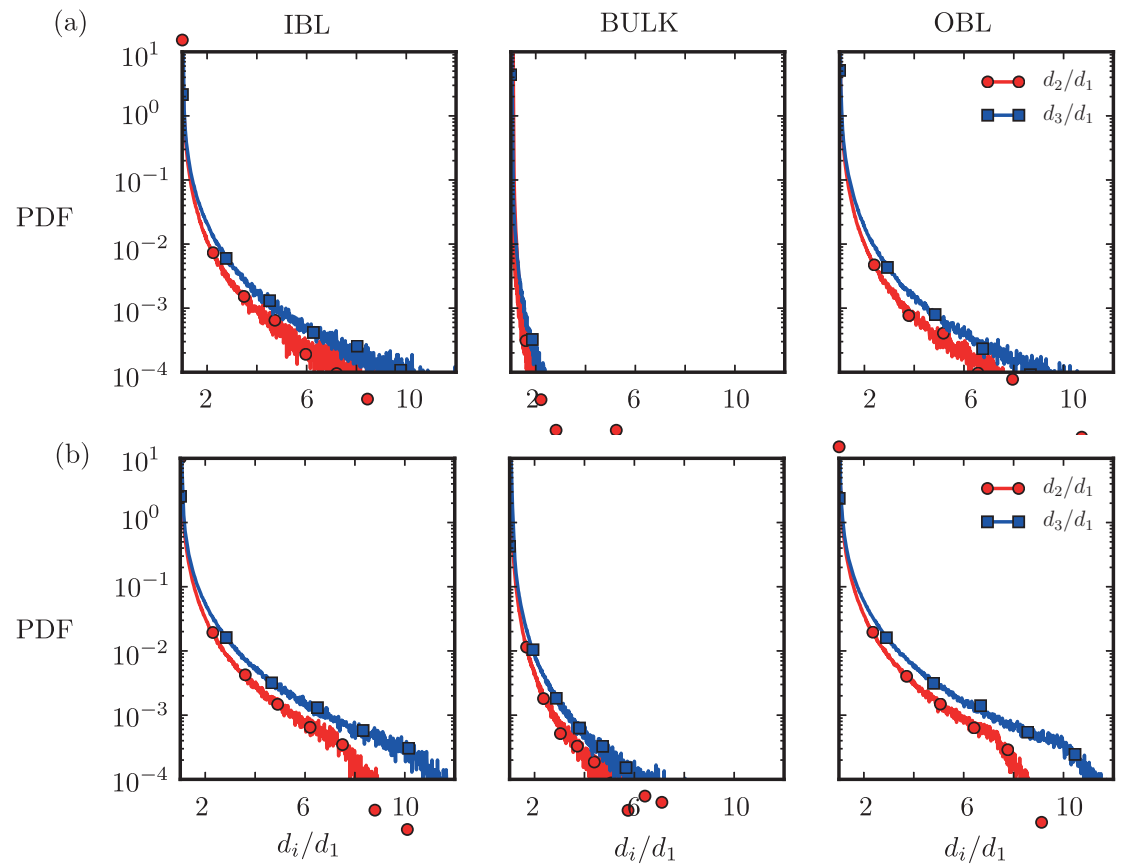

Figure 3.10: Probability distribution function (PDF) of the ratio of semi-axes $\left(d_{1}<d_{2}<d_{3}\right)$ for $R e_{i}=2500$ and $\hat{\mu}=100$ (a) $C a=0.05$ (b) $C a=0.2$. 
(a)
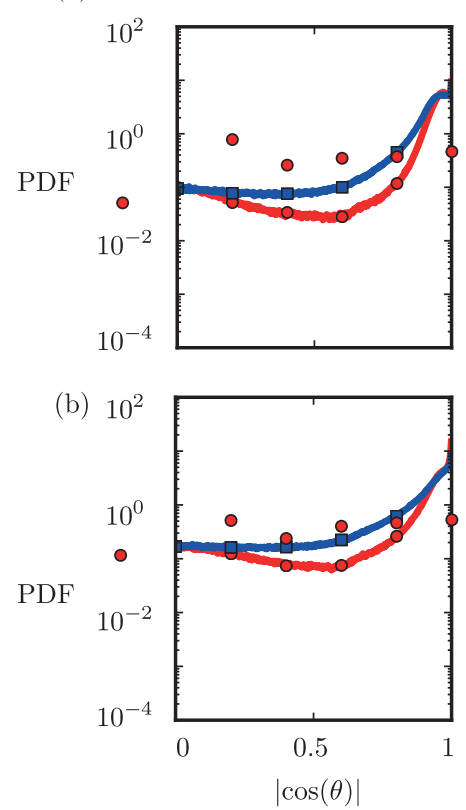

BULK
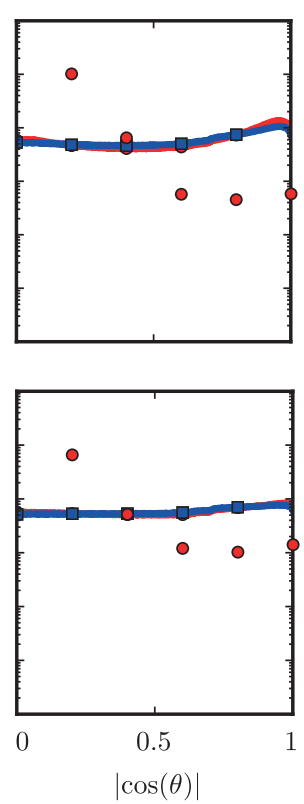

OBL
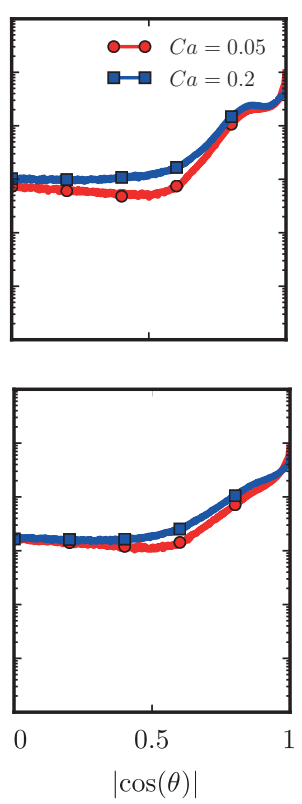

Figure 3.11: Probability distribution functions (PDF) of the stream-wise orientation of the major axis of droplets for $C a=0.05$ and $C a=0.2$ and $\hat{\mu}=100$ (a) $R e_{i}=2500$ (b) $R e_{i}=5000$. 
straining direction due to the effect of rotation. This effect is attributed to the vorticity sweeping the material elements (in our case the axes of the ellipsoidal drop) away from the maximum strain-rate direction along with the rotation of the principal strain-rate axes relative to the material lines. Given that we are studying a highly inhomogeneous and anisotropic wall sheared flow, the former effect is more pronounced and is the cause of observed droplet shapes. More recently Johnson et al. [92] calculated the Cramèr function for Finite Time Lyapunov Exponents (FTLE) of Lagrangian particles in isotropic turbulence and by isolating the effect of the strain-rate tensor they concluded that the deformation of a fluid volume is higher when the immersed particle does not rotate which resonates very well with the deformation statistics of highly viscous drops we observe in our study.

To understand the influence of rotation on the relative orientation of the drops we plot PDF's of the direction cosine of the major axis with the stream-wise direction in figure 3.11 for two different Reynolds numbers and $\hat{\mu}=100$. It can be immediately observed that similar to $\hat{\mu}=1$ the orientation is highly isotropic in the bulk region even for $\hat{\mu}=100$. In the IBL and OBL, while there is a tendency for the major axis to align with the stream-wise direction which is similar to what we observed for $\hat{\mu}=1$, the distribution of the orientation for $\hat{\mu}=100$ is more uniform than $\hat{\mu}=1$ which can be explained from earlier discussions about the increased influence of rotation.

From figures 3.7, 3.8 and it is clear that the orientational behaviour of continuously deforming ellipsoidal droplets is completely different from that of solid axis-symmetric ellipsoids. For both viscosity ratios, the capillary number has negligible influence on the rotational behaviour of the droplets, suggesting that the droplets adjust to the underlying flow conditions regardless of the degree of deformation.

\subsection{Summary}

In this chapter we study the influence of capillary number $C a$ and viscosity ratio $\hat{\mu}$ on the deformation and orientation statistics of neutrally buoyant sub-Kolmogorov drops dispersed into a turbulent Taylor-Couette (TC) flow. The drops are considered to be tri-axial ellipsoids which allows us to mathematically represent the surface of the drop using a symmetric second-order positive definite tensor. By coupling the phenomenological model proposed by Maffettone and Minale [68] for ellipsoidal drops with Lagrangian tracking and DNS for the carrier flow, we solve the time dependent evolution equation of the shape tensor of approximately $10^{5}$ drops dispersed into a turbulent TC flow. We find that increasing the capillary number results in large deformations of the ellipsoidal droplets due to weakening surface tension forces. 
Additionally, the drops deform more when they are in the inner and outer boundary layer as compared to the bulk region. Due to increased influence of rotation with increase in viscosity ratio, the droplets tend to be more oblate or disk-like as compared to more prolate or cigar-like droplets for low viscosity ratio systems. Regardless of the capillary number $C a$ and viscosity ratio $\hat{\mu}$, the major axis of the drop tends to align with the stream-wise direction in the inner and outer boundary layer while the orientation is highly isotropic in the bulk; a behaviour similar to solid ellipsoidal particles in a turbulent channel flow. However, the preference of orientation with the eigen directions of strain rate tensor of these drops is very different from those exhibited by axis-symmetric ellipsoids in an isotropic turbulent flow. This is a result of the continuously deforming nature of the ellipsoidal drops dispersed into a highly inhomogeneous anisotropic flow. 
DEFORMATION AND ORIENTATION STATISTICS OF SUB-KOLMOGOROV DROPS 


\section{Chapter 4}

\section{Deformable bubbles with enhanced Euler-Lagrange tracking}




\subsection{Introduction}

Deformability of drops or bubbles dispersed in a moving fluid plays a very important role in the overall behaviour of multi-phase flows. The extent of deformability which is determined by the competing actions of viscous, inertia and surface tension forces can affect a bubble/drop's motion (rectilinear, spiral or zigzag) [93-97], alter the drop's or bubble's migration or clustering patterns $[12,14,15]$ and in some cases also the net momentum or heat transfer in a multi-phase flow $[4,22,98,99]$. It is thus of paramount importance to understand the influence of deformability in multi-phase flows given its wide range of applications (for example bubble columns [100], drag reduction in liquefied gas transport and naval industry [8, 101] etc.).

In this chapter, we build upon the techniques used in $\S 3$ by coupling the sub-grid deformation model with two-way coupled Euler-Lagrange tracking which is referred to as enhanced Euler-Lagrange tracking. This allows us to study deformable sub-Kolmogorov bubbles dispersed in a turbulent flow.

The numerical consequences of having a dispersed phase whose size is of the same or smaller than the Kolmogorov scale has been discussed in detail in $\S 1$ and $\S 3$. Here, we briefly go through these consequences and also possible alternative which would allow us to simulate multiphase flows with deformable sub-Kolmogorov dispersed phase. When the size of the dispersed phase is sub-Kolmogorov fully resolved simulations become unrealistic due to the extremely fine grids required to resolve each drop/bubble. In such cases, two-way coupled Euler-Lagrange simulations (point-particle approach) can be employed since then the dispersed phase need not be resolved any more but the acceleration of individual particles, bubbles or drops is computed through empirical force correlations [45].

Over the last few decades point-particle approaches have been used successfully in the field of multiphase flows given its extremely versatile and computationally inexpensive nature. In particular, studies have focussed on understanding the influence of approximately $10^{5}$ sub-Kolmogorov particles, drops or bubbles on a turbulent carrier flow [25, 26, 42, 45, 74, 76, 83-85, 102-107]. However, in contrast to fully resolved approaches, point-particle Euler-Lagrange tracking does not allow for deformation or orientation dynamics which limits its versatility.

There is a need for a technique which can handle large numbers $\left(O\left(10^{5}\right)\right)$ of sub-Kolmogorov drops or bubbles in highly turbulent flows while allowing for deformation and orientation dynamics to be present. To this effect, in this chapter we first build upon the currently existing point-particle approach to account for deformability by coupling traditional Euler-Lagrange tracking with a sub-grid 
deformation model which was introduced in $\S 3$. We also employ two-way coupling in between the dispersed phase and the carrier phase which allows us to study their influence on each other. With this enhanced two-way coupled point-particle approach we are able to simulate approximately $10^{5}$ continuously deforming sub-Kolmogorov bubbles dispersed in a turbulent flow field. In particular, we focus on the effect of deformability of low-density bubbles on the net friction or drag in a turbulent Taylor-Couette flow. Here it is important to remember that given the sub-Kolmogorov nature of the dispersed phase, numerical simulations of such large-scale multiphase systems which can also account for deformability can only be possible through the enhanced two-way coupled Euler-Lagrange approach introduced in this chapter.

Several numerical and experimental studies have demonstrated that injection of a small amount of dispersed phase such as bubbles can lead to strong drag reduction effects in TC flow $[4,18,19,26,74]$. Here, drag reduction refers to a decrease in the net torque required to keep the cylinders rotating at a specific angular velocity. The extent of drag reduction, flow modification and the physical mechanisms at play depends on the specific flow regime.

When the Reynolds number in the flow is such that boundary layers and bulk of the flow are laminar and turbulent, respectively, the coherent structures such as Taylor rolls play a dominant role in the angular momentum transport. In this regime, sub-Kolmogorov spherical bubbles $\left(d_{b}<\eta_{K}\right)$ are highly effective in reducing the overall drag on the rotating cylinders (cf. §2) [74]. However, the drag reduction effects induced by sub-Kolmogorov spherical bubbles reduce drastically with increasing Reynolds numbers. In the highly turbulent regime (i.e. turbulent boundary layers and bulk), experimental studies have shown that large bubbles $\left(d_{b}>>\eta_{K}\right)$ which can deform are necessary to recover the drag reduction effects observed in the previous smaller $R e_{i}$ regime [4, 22]. Beyond this, there is a lack of clear understanding on the relevant parameters and conditions crucial for strong drag reduction and also the influence of the shape, size, orientation and accumulation of the dispersed bubbles/drops on the flow.

We consider the shape of the dispersed phase to be deformable tri-axial ellipsoids and compute the deformation through a sub-grid phenomenological model which is described in the next section. As mentioned earlier, our focus here is to understand the effect of deformability of sub-Kolmogorov bubbles on the overall drag on the driving cylinder in TC flow. Additionally, we will look at how deformability affects the mean distribution of the bubbles in the domain. We also answer the question of how these bubbles deform and orient in various regions of the flow (boundary layers and bulk) and how this impacts their effect on the net driving torque in the flow. 
The chapter is organised as follows: In the next section we will detail the enhanced two-way coupled Euler-Lagrange approach which brings together various numerical ingredients to allow for simulations of $10^{5}$ deformable ellipsoidal bubbles. In section 4.3 we discuss the results obtained from our simulations and subsequently provide a summary and outlook of the paper in section 4 .

\subsection{Governing Equations}

\subsubsection{Dynamics of carrier phase and shape tensor}

The dynamics of the carrier phase is solved using direct numerical simulations (DNS) of the Navier-Stokes equations in cylindrical coordinates as detailed in $§ 1,2,3$. A second-order accurate finite-difference scheme with fractional time stepping is used for the spatial and temporal discretisation of equations (1.1) and (1.2) [30, 31]. A non-uniform grid spacing is employed in the radial (wall-normal) direction and periodic boundary conditions are used in the azimuthal (stream-wise) and axial (span-wise) directions.

We consider the dispersed phase to consist of deformable tri-axial ellipsoidal bubbles $\left(\rho_{b} / \rho=0.001\right)$ while the extent of deformation of an individual bubble depends on the local flow conditions. The scheme to solve the deformation dynamics of the ellipsoidal bubbles is detailed in $\S 3[67,68,108]$.

The non-dimensional deformation model described in $\S 3$ gives rise to the control parameter $C a$ which is the capillary number and is the ratio of the viscous forces acting on an individual bubble relative to its inherent surface tension forces. The capillary number can be expressed as the ratio of the interfacial relaxation time scale $\tau=\mu_{c} R / \sigma$ to the characteristic flow time scale $\tau_{\eta}$ i.e. $C a=\tau / \tau_{\eta}=\mu R G / \sigma$. When $C a<<1$ the deformation dynamics are decoupled from the turbulent fluctuations in the carrier flow and the influence of the instantaneous deformation on the flow can be neglected. In such a case the forcing from the dispersed phase onto the carrier phase $\left(\bar{f}_{b}(\bar{x}, t)\right.$ in equation (1.1)) is computed as $\bar{f}_{b}(\bar{x}, t)=\sum_{i=0}^{N_{b}}\left(\frac{D \bar{u}}{D t}-\bar{g}\right) V_{p} \delta\left(\bar{x}-\bar{x}_{b}(t)\right) ; N_{b}$ is the total number of bubbles dispersed into the flow, $V_{b}$ is the volume of an individual bubble and $\bar{x}_{b}(t)$ its position $[5,26,42,74]$. The function $g\left(\overline{\bar{S}}^{*}\right)=3 I I I_{s} / I I_{s}$ in equation (3.3) ensures volume conservation of each bubble $\left(I I_{s}\right.$ and $I I I_{s}$ are the second and third invariant of the shape tensor, respectively); $f_{1}$ and $f_{2}$ are parameters which depend only on the viscosity ratio $\hat{\mu}=\mu_{d} / \mu_{c} ; f_{1}=40(\hat{\mu}+1) /((2 \hat{\mu}+3)(19 \hat{\mu}+16))$ and $f_{2}=5 /(2 \hat{\mu}+3)$. For additional details on the function $g\left(\overline{\bar{S}}^{*}\right)$ and the formulations of $f_{1}, f_{2}$ the reader is referred to $\S 3$ and the following works $[67,68,108]$. 


\subsubsection{Modelling of effective forces}

We now describe the governing equations used to compute the hydrodynamic forces acting on the ellipsoidal bubbles. In Euler-Lagrange tracking the forces acting on the dispersed phase depend on the local flow conditions in the carrier phase and are computed through empirical correlations. Here it is important to point out that the formulation of the forces acting on a deformable tri-axial ellipsoidal particle in a generalised flow field is highly non-trivial. The forces depend on a large number of parameters such as the instantaneous particle Reynolds number, lengths of the three semi-axes, relative orientation of the semi-axes with the flow direction, time scale of deformation, anisotropic added-mass effects etc. to name a few. When the ellipsoids are rigid, a number of studies starting from the seminal work of Jeffery [109] have tried to predict the translational and rotation dynamics of the ellipsoids under given flow conditions [83, 85, 104-106, 110-117]. The modelling is further simplified when the ellipsoids are symmetric (i.e. prolate or oblate) and two of the semi-axes are approximately equal in length.

In this work we adopt the formulations used by Yin et al. [105] and more recently by Njobuenwu et al. [85] who studied the motion of anisotropic particles in unsteady non-uniform flow conditions. While the correlations for drag and lift used in the above mentioned studies are applicable for rigid ellipsoidal particles, they can be adopted for deforming ellipsoidal bubbles under certain conditions. The first condition is that the bubble interface is fully contaminated with impurities or surfactants so that the interfacial boundary condition is no-slip which is similar to that of the rigid particles. The second condition is that the bubbles are sub-Kolmogorov and the capillary number $\mathrm{Ca}<<1$, i.e. the characteristic flow time scale is much larger than the interfacial relaxation time scale. In such a case the instantaneous drag and lift force experienced by the ellipsoidal bubble would be approximately similar to that of the rigid particle. Additionally, the correlations used by Yin et al. [105] and Njobuenwu et al. [85] are primarily for axis symmetric ellipsoids, while the shape tensor solved in equation (3.3) corresponds to a tri-axial ellipsoid. In order to circumvent this problem we keep the maximum capillary number of the dispersed phase limited to $C a=0.1$. By limiting the capillary number we ensure that the deformed ellipsoids are close to being axisymmetric and this will be shown in more detail in the next section. Under these conditions, the momentum equation for the dispersed phase which consists of contributions from drag, lift, added mass and buoyancy can be written as follows:

Extension to slip boundary conditions is possible, so it is not a strict condition. 


$$
\begin{aligned}
\rho_{p} V_{p} \frac{d \bar{v}}{d t}=0.5 \rho A_{D} C_{D}|\bar{u}-\bar{v}|(\bar{u}-\bar{v}) & +0.5 \rho A_{L} C_{L} \frac{\hat{e}_{3}(\bar{u}-\bar{v})}{|\bar{u}-\bar{v}|}\left(\hat{e}_{3} \times(\bar{u}-\bar{v})\right) \times(\bar{u}-\bar{v}) \\
& +\rho V_{p} C_{A}\left(\frac{D \bar{u}}{D t}-\frac{d \bar{v}}{d t}\right)+\rho V_{p}\left(\frac{D \bar{u}}{D t}-\bar{g}\right) \\
& +\rho_{p} V_{p} \bar{g}
\end{aligned}
$$

The first expression on the right hand side of equation (4.1) accounts for the drag force, the second for the lift force and the last two represent the added-mass force and buoyancy, respectively. In equation (4.1), $\hat{e}_{3}$ is the normal vector in the direction of the major-axis, $A_{D}$ and $A_{L}$ are the projected areas normal to the direction of drag and lift, respectively while $\bar{u}$ and $\bar{v}$ are the velocities of the fluid and the bubble, respectively. The projected areas are computed as $A_{D}=\pi R^{2}\left(\cos ^{2} \alpha+\left(4 d_{31} / \pi\right)^{2} \sin ^{2} \alpha\right)^{1 / 2}$ and $A_{L}=\pi R^{2}\left(\sin ^{2} \alpha+\left(4 d_{31} / \pi\right)^{2} \cos ^{2} \alpha\right)^{1 / 2} ; \alpha$ is the angle between the largest semi-axes and the relative slip velocity $(\bar{u}-\bar{v})$ and $d_{31}=d_{3} / d_{1}$ is the ratio between the largest $\left(d_{3}\right)$ and smallest $\left(d_{1}\right)$ semi-axes.

The most important components of equation (4.1) are $C_{D}$ and $C_{L}$ which are the drag and lift coefficients, respectively and are modelled as follows [85, 105]:

$$
\begin{gathered}
\frac{C_{D}}{K_{2}}=\frac{24}{R e_{b} K_{1} K_{2}}\left(1+0.118\left(R e_{b} K_{1} K_{2}\right)^{0.6567}\right)+\frac{0.4305}{1+\frac{3305}{R e_{b} K_{1} K_{2}}} \\
C_{L}=C_{D} \sin ^{2} \alpha \cdot \cos \alpha
\end{gathered}
$$

$R e_{b}=2 R|\bar{u}-\bar{v}| / \nu$ is the bubble Reynolds number calculated based on the initial undistorted radius of the bubble. $\alpha$ is the angle between $\hat{e}_{3}$ and the relative velocity $u-v, K_{1}=\frac{1}{3} d_{n} /(2 R)+\frac{2}{3} \psi^{-0.5}$ and $K_{2}=10^{1.8148(-\log \psi)^{0.5743}}$ are coefficients which depend on the particle sphericity $\psi$ which is the ratio of the surface area of an undeformed particle to the surface area of the deformed particle. $d_{n}=\left(4 A_{D} / \pi\right)^{1 / 2}$ is the diameter of a circle having the same projection area as that of the deformed particle. While there are several formulations of the lift force and the lift coefficient, here we choose the one proposed by Hoerner [118] which assumes that the lift is proportional to the drag force and depends on the relative orientation of the ellipsoid major-axis with the slip velocity and the lift coefficient is computed as give in equation (4.3).

The added mass coefficients are computed based on the ratio of the semi-axes $d_{31}$ as computed by Lai and Mockros for axisymmetric ellipsoidal bubbles [119], 


$$
C_{A}=\frac{d_{31} \ln \left(d_{31}+\sqrt{d_{31}^{2}-1}-\sqrt{d_{31}^{2}-1}\right.}{d_{31}^{2} \sqrt{d_{31}^{2}-1}-d_{31} \ln \left(d_{31}+\sqrt{d_{31}^{2}-1}\right)} .
$$

While in principle the added mass coefficient for a tri-axial ellipsoid is a second-order tensor, given the complex and intractable nature of the problem here we consider the simplified form of a fully isotropic coefficient as shown above.

\subsubsection{Simulation parameters}

In equation (4.1), we set the density ratio of the dispersed phase as $\hat{\rho}=\rho_{p} / \rho=$ 0.001 and the viscosity ratio in equation (3.3) to $\hat{\mu}=\mu_{d} / \mu_{c}=0.01$. Two different inner cylinder Reynolds numbers of $R e_{i}=2500$ and $R e_{i}=8000$ are considered and the radius ratio and aspect ratio of the set up is fixed to $\eta=r_{i} / r_{o}=0.833$ and $\Gamma=4$, respectively. It has been ensured that increasing the aspect ratio beyond the chosen value of $\Gamma=4$ does not influence the results or statistics discussed in later sections. The capillary number of the dispersed bubbles is varied from $C a=10^{-6}$ which corresponds to the spherical-limit (i.e. the dispersed bubbles are un-deformed and fully spherical) to $C a=10^{-1}$. A volume fraction of $\alpha_{g}=0.1 \%$ bubbles is used in all simulations and periodic boundary conditions are employed for the bubbles in the azimuthal and axial directions. To treat the collision between the wall and the ellipsoids an elastic-collision model based on the bounding box approach as described in $\S 3[108]$ is used here.

\subsection{Results}

\subsubsection{Global transport quantities}

We will first look at the effect of the bubbles on the global properties of the flow i.e. the driving torque on the cylinders. In order to do so, we compute the torques required to sustain the flow at a specific Reynolds number in both the single phase $\left(\tau_{s}\right)$ and two-phase $\left(\tau_{t}\right)$ systems and compute the overall drag reduction (in \%) induced by the dispersed bubbles as $D R=100\left(\tau_{s}-\tau_{t}\right) / \tau_{s}$. The driving torques can also be converted into a skin-friction coefficient as $C_{f}=\sigma_{w} / 0.5 \rho U_{i}^{2}$, where $\sigma_{w}$ is the averaged wall-shear stress. Before analysing the effect of deformability of a sub-Kolmogorov dispersed phase in TC flow, it is important to understand the origin of drag reduction in the low capillary number limit, i.e. when the surface tension forces in the bubbles are very large compared to the viscous forces and the bubbles are close to spherical 
(a)

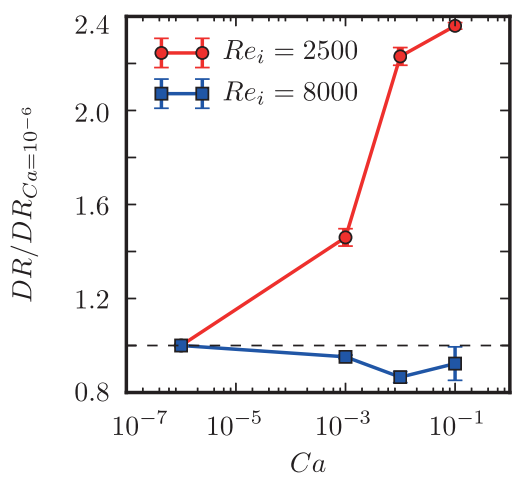

(b)

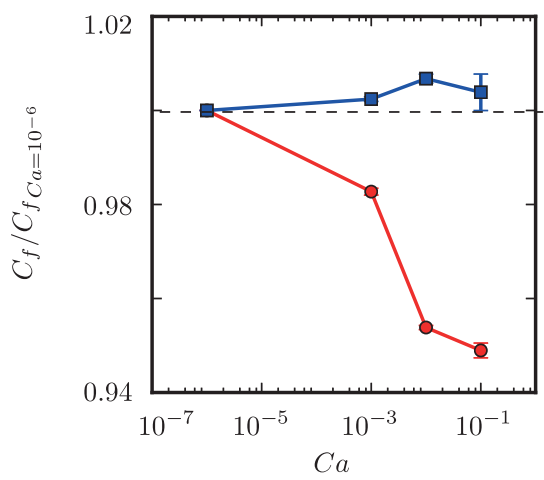

Figure 4.1: (a) Drag reduction $(D R)$ and (b) Skin friction coefficient $C_{f}$ versus Capillary number $\mathrm{Ca}$ of the dispersed phase for two different Reynolds numbers

which has been detailed in $\S 2$. In such a system it has been shown that the buoyancy of the bubbles disrupt and weaken the plume ejections from the inner cylinder and consequently the Taylor rolls which effectively transfer momentum and this leads to drag reduction [74]. Given that the weakening of the Taylor rolls is just due to the buoyancy of the bubbles dispersed into the flow, we expect that this mechanism is still relevant when the bubbles become deformable. Here, it is important to remember that the system is incompressible and thus deformability has no effect on the overall buoyancy of the bubbles. We now quantitatively compare the drag reduction obtained by spherical and deformable bubbles.

To understand the effect of deformability, we plot the normalised drag reduction and skin-friction coefficient versus the capillary number of the dispersed phase for two different Reynolds numbers in figure 4.1 (a) and (b), respectively. To show the effect of deformation, both $D R$ and $C_{f}$ are normalised using their corresponding values at $C a=10^{-6}$, i.e. in the spherical limit of the bubbles. At $R e_{i}=2500$, one can observe that with increasing capillary number there is a net increase in drag reduction in comparison to the low Capillary number $\left(C a=10^{-6}\right)$ limit. In other words, by weakening the inherent surface tension forces of the sub-Kolmogorov bubbles we are able to more than double the net drag reduction achieved in the flow. In figure 4.1 (b), we plot the same data but in the form of the normalised skin-friction coefficient which decreases with increasing $\mathrm{Ca}$ for $R e_{i}=2500$. At this Reynolds number it is clear that the drag reduction achieved in the low Capillary number limit can be further enhanced by making the dispersed bubbles deformable. At $R e_{i}=8000$, there is barely any 


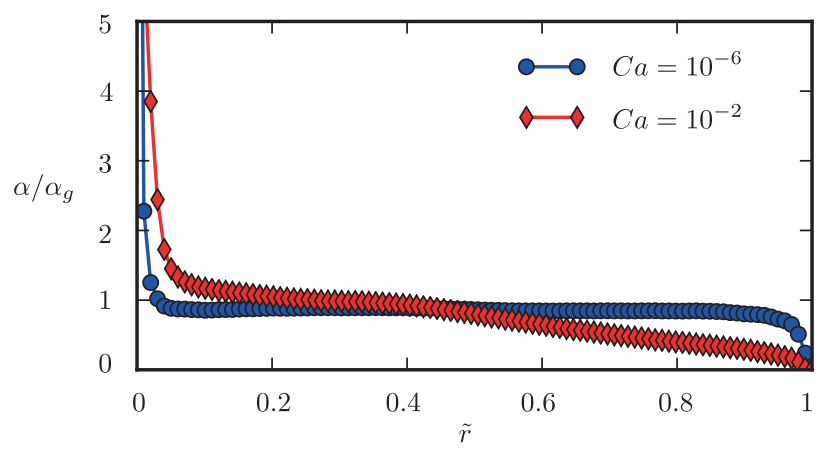

Figure 4.2: Radial (wall-normal) profiles of azimuthally, axially and time averaged local volume fraction of the dispersed phase for two different Capillary numbers at $R e_{i}=2500$. The profiles for $C a=10^{-3}$ and $C a=10^{-1}$ lie below and above the profile of $C a=10^{-2}$, respectively and are not shown here for clarity.

significant change in the drag with increasing deformation. When sub-Kolmogorov spherical bubbles are used at such high Reynolds numbers, negligible drag reduction has been observed as shown in previous studies [18, 26, 74]. At $R e_{i}=8000$, the coherent structures lose importance in the angular momentum transport and the turbulent fluctuations start taking over. In such cases, the buoyancy of the dispersed phase is not strong enough in comparison to velocity fluctuations such that it can alter the flow structures and reduce dissipation to achieve drag reduction. We observe in figure 4.1 that then deformability of the dispersed phase has no significant effect on the net drag reduction achieved by a sub-Kolmogorov dispersed phase at high Reynolds numbers. This is justified given the incompressibility of the two-phase system and thus deformability has no effect on the overall buoyancy of the dispersed phase.

\subsubsection{Mean bubble concentration profiles}

We now focus on the simulations at $R e_{i}=2500$ to understand the increase in drag reduction with deformation. In figure 4.2 we plot the radial (wall-normal) profiles of the normalised mean local volume fraction of the dispersed phase. The wall-normal position is normalised using the gap-width as $\tilde{r}=\left(r-r_{i}\right) / d$; thus $\tilde{r}=0$ refers to the bubbles close to the inner cylinder while $\tilde{r}=1$ refers to the bubbles close to the outer cylinder. As has been shown in previous studies [18, 26, 74], rigid spherical bubbles in TC flow $\left(\mathrm{Ca}=10^{-6}\right)$ tend to accumulate near the inner cylinder as a result of the heavier carrier fluid being pushed away by the centrifugal forces. However, as 


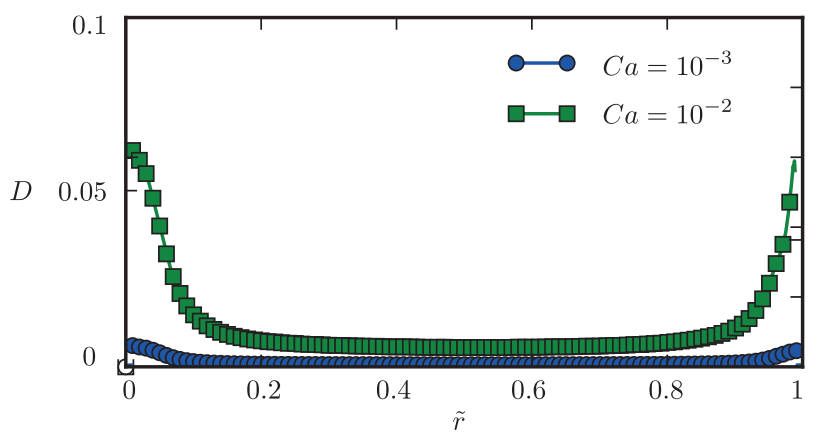

Figure 4.3: Radial (wall-normal) profiles of azimuthally, axially and time averaged deformation parameter of the bubbles for two different Capillary numbers at $R e_{i}=2500$.

can be seen from figure 4.2, the capillary number of the dispersed phase seems to play a very important role in the relative distribution of the bubbles in the domain for $R e_{i}=2500$. While the distribution is close to homogeneous in the bulk for low capillary number, there appears to be a gradient in the bubble distribution at the higher $\mathrm{Ca}$. More bubbles tend to accumulate near the inner cylinder with increasing Capillary number. The enhanced drag reduction with increasing capillary number observed in figure 4.1 can be related to an increase in accumulation of the dispersed bubbles near the inner cylinder. As discussed earlier, when rigid spherical bubbles are injected into the TC system, buoyancy is crucial in disrupting and weakening the plumes ejections which are responsible for the angular momentum transport in the wall-normal direction. A larger concentration of bubbles near the inner cylinder implies a stronger effect of the dispersed phase on the plumes being ejected from the inner cylinder which results in an increase in drag reduction.

The mean position of the bubbles dispersed in the TC domain is governed by the hydrodynamics forces that act on them which in turn depend on the shape of the bubbles as discussed in the previous section. It thus becomes important to look at the extent of deformation of bubbles in different regions of the flow. For this we use the deformation parameter $D=\left(d_{3}-d_{1}\right) /\left(d_{3}+d_{1}\right)$ which gives an idea of the extent of deformation of individual bubbles. Here $d_{3}$ and $d_{1}$ are the lengths of the major and minor axis of the ellipsoid, respectively [58, 59]; for $C a=0$ we have $D=0$ by definition. In figure 4.3 , we plot the azimuthally, axially and time averaged profiles of the deformation parameter $D$ versus the normalised wall-normal position $\tilde{r}$ which shows a clear spatial inhomogeneity in the extent of deformation of the dispersed 

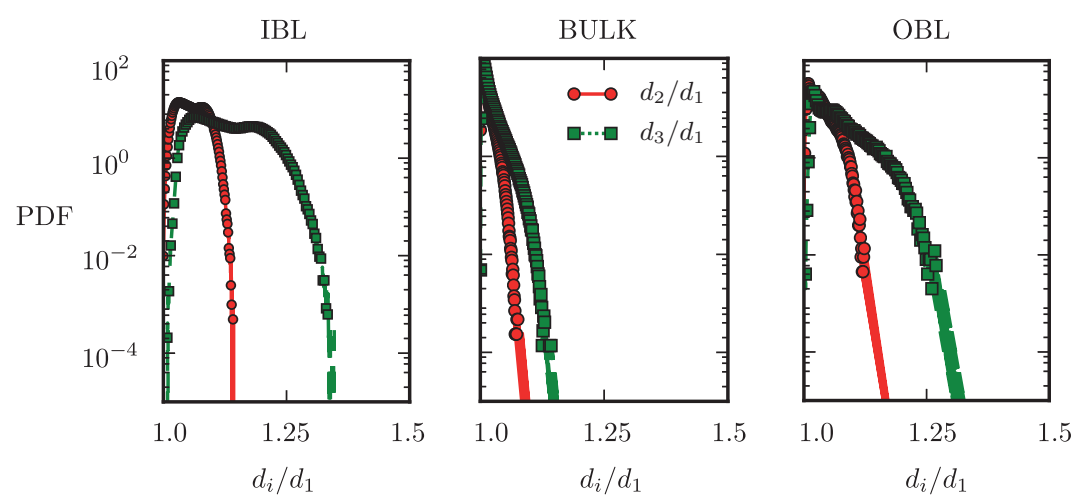

Figure 4.4: Probability distribution functions of the ratio of semi-axes of bubbles in the inner boundary layer (IBL), bulk, and outer boundary layer (OBL) for $C a=10^{-2}$ at $R e_{i}=2500$.

phase. When the Capillary number is high, the bubbles near the walls deform much more than those in the bulk due to the strong shear in the boundary layers. For both capillary numbers the deformation is relatively lower and homogeneous in the bulk region of the flow which is a result of relatively lower shear provided by the Taylor rolls. Here it is also important to note that the deformation statistics of the bubbles is similar to neutrally buoyant drops dispersed in TC flow [108]. This is because the deformation is fully governed by equation (3.3) which depends only on the local flow conditions and the inherent surface tension of the dispersed phase and not on buoyancy effects. This is justified given the sub-Kolmogorov nature of the bubbles and that buoyancy effects on the deformation only become relevant when the dispersed phase is much larger than the Kolmogorov scale.

\subsubsection{Bubble shape and orientation}

While the deformation parameter gives a good idea on the spatial inhomogeneity of the deformation, it does not give any information on the exact shape of the bubbles in various regions of the flow. In order to understand this more clearly we compute the probability distribution functions (PDF) of the ratios of the ellipsoid axes i.e. $d_{2} / d_{1}$ and $d_{3} / d_{1}\left(d_{1}<d_{2}<d_{3}\right)$ in three different regions of the flow, namely the inner boundary layer (IBL), in the bulk and in the outer boundary layer (OBL). These PDF's give us a better idea on whether the dispersed bubbles are more prolate $\left(d_{3} / d_{1}>1\right.$ and $\left.d_{2} / d_{1} \sim 1\right)$ or oblate $\left(d_{3} / d_{1} \sim d_{2} / d_{1}>1\right)$. It has already been seen 

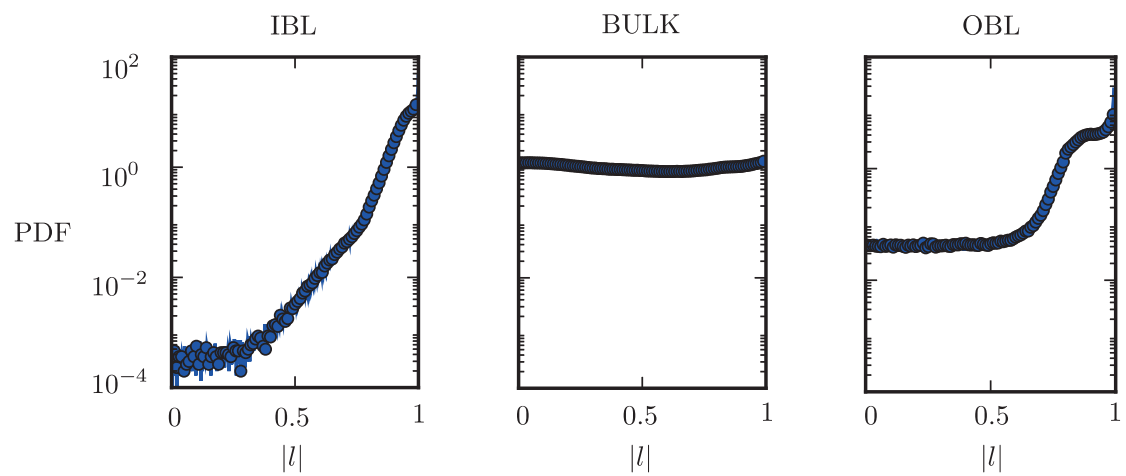

Figure 4.5: Probability distribution functions of the direction cosine of the orientation of the semi-major axes of the bubbles with the azimuthal (stream-wise) direction in the inner boundary layer (IBL), bulk, and outer boundary layer (OBL) for $C a=10^{-2}$ at $R e_{i}=2500$.

in the case of neutrally buoyant drops that an increased influence of rotation over stretching can result in oblate shaped drops while prominence of stretching results in more prolate shaped drops (cf. §3) [108]. The PDF's for the case of $R e_{i}=2500$ and $C a=10^{-2}$ are shown in figure 4.4. As can be seen from the distributions in the IBL and OBL, the bubbles are close to being prolate which indicates a stronger influence of stretching over rotation of the bubbles. We also observe from the distribution of $d_{2} / d_{1}$ that a large number of bubbles distributed throughout the domain are close to being axisymmetric i.e. $d_{2} / d_{1} \sim 1$. For consistency of our model this is an important observation since the momentum equation used for the dispersed bubbles (equation 4.1 ) is applicable primarily for axi-symmetric particles. When the capillary number of the bubbles goes beyond $C a=0.1$ the shapes of the dispersed bubbles become fully tri-axial (i.e. all three semi-axes are unequal in length) and in such a case equation (4.1) would need further refinement.

We now look at the distributions of the orientations of the bubbles in the flow. In figure 4.5 we plot the PDF of the direction cosine of the orientation of the bubbles with $\hat{\theta}$ (azimuthal) direction, i.e. $l=\cos \left(\theta_{\hat{e}_{3} \hat{\theta}}\right)$ where $\theta_{\hat{e}_{3} \hat{\theta}}$ is the angle between $\hat{\theta}$ and the semi-major axis $\left(\hat{e}_{3}\right)$ of an individual bubble. In the IBL we notice that the majority of the bubbles tend to orient in stream-wise direction. A relatively weaker but observable preferential orientation is also found in the OBL while it is highly isotropic in the bulk due to the Taylor rolls. Similar distributions are observed for neutrally buoyant drops dispersed into TC flow [108] and in the case of solid ellipsoidal particles in a turbulent channel flow [83-85]. The orientation of the bubbles with the 

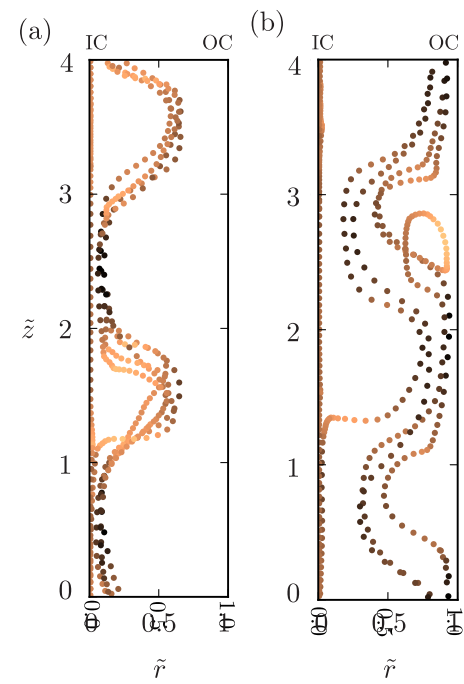

Figure 4.6: Trajectories of randomly chosen bubbles in the $\hat{r}-\hat{z}$ plane taken over approximately 100 large eddy turn over times (a) $C a=10^{-2}$ (b) $C a=10^{-3}$ at $R e_{i}=2500$. IC refers to inner cylinder while $\mathrm{OC}$ refers to the outer cylinder. The colour scale indicates the axial velocity of the bubble where a dark colour indicates a higher velocity while a lighter colour indicates a lower velocity.

stream-wise direction is assisted by the stretching provided by the strong shear in the IBL which results in prolate shaped bubbles (c.f. figure 4.4). Such an alignment preference also minimises the projected area $\left(A_{D}\right)$ of the bubble in the direction of the stream-wise drag force. Given the sub-Kolmogorov nature of the dispersed phase the bubble Reynolds numbers $R e_{b}$ is $O(1)$ in these systems (c.f. figure 2.9 in $\S 2$ ). This suppresses any instabilities that may arise in the bubble motion which tends to change its preferential orientation.

We now go back to explaining the preferential accumulation of bubbles near the inner cylinder with increasing capillary number as shown in figure 4.2. From figures 4.4 and 4.5 we observed that the bubbles close to the inner cylinder for $C a=10^{-2}$ are prolate and also align themselves with the stream-wise direction. While this reduces the overall projected area of the deformed bubbles in the azimuthal (stream-wise) direction it increases the projected area in the radial (wall-normal) direction. To understand the effect of this increased projected area on the overall motion of the bubbles we plot the trajectory of randomly chosen bubbles in the $\hat{r}-\hat{z}$ plane for two different capillary numbers in figure 4.6. We also refer to figure 2.8 of $\S 2$ which shows the effect of buoyancy and Reynolds numbers on the trajectories of buoyant spherical 
bubbles in TC flow. In both figures, one can clearly see that bubbles enter the bulk from the plume ejection regions near the inner cylinder $(\tilde{r}=0$ and $\tilde{z} \sim 1, \tilde{z} \sim 3$ in figure 4.6) and continue on their upward motion which is assisted by buoyancy. The bubbles moving in the wall-normal direction from the inner cylinder into the bulk are hampered by the increased drag force from the increased projected area which results in their preferential accumulation close to the inner cylinder when they become deformable. As discussed earlier, given the incompressibility of the overall system deformation of the bubbles has no effect on its buoyancy and a larger number of bubbles near the inner cylinder means that the weakening of the plumes by the bubbles is stronger; this in turn leads to stronger drag reduction.

\subsection{Summary}

In this chapter we have brought together direct numerical simulations of Taylor-Couette (TC) flow (a wall-bounded sheared system), force-based Lagrange tracking of an ellipsoidal dispersed phase and a sub-grid deformation model with which we have improved the conventional Euler-Lagrange tracking widely used in the study of multi-phase flows. This two-way coupled enhanced Euler-Lagrange approach allows us to track $O\left(10^{5}\right)$ sub-Kolmogorov deformable drops and bubbles in a turbulent flow field and also study their influence on the global and local properties of the flow. The simulations performed using this approach are presently impossible with fully-resolved techniques due to the sub-Kolmogorov nature of the dispersed phase.

We have used the numerical simulations to understand the effect of deformability of approximately $10^{5}$ bubbles dispersed into TC flow and in particular we focus on their effect on the overall drag on the rotating cylinder. We find that at an inner cylinder Reynolds number of $R e_{i}=2500$, increasing the deformability (i.e. the capillary number) results in an increase in the overall drag reduction. By studying the mean bubble volume fraction profiles in the domain, we find that deformable bubbles prefer to accumulate near the rotating inner cylinder. The PDF's of the ellipsoid axes and their orientational preference indicate that near the inner cylinder the bubbles are primarily prolate and tend to align with the stream-wise direction. This results in an increase in the drag experienced by the near-wall deformed bubbles in the wall-normal direction which ultimately results in their accumulation near the inner cylinder. Drag on the inner cylinder is directly connected to the strength of plume ejections which are disrupted more effectively by deformable bubbles due to their preferential accumulation.

At a relatively higher Reynolds number of $R e_{i}=8000$ we find that deformability of 
sub-Kolmogorov bubbles has no effect on drag reduction as the turbulent fluctuations are much stronger in comparison to the buoyancy of the bubbles. At this Reynolds number and beyond, i.e. when both boundary layers and bulk become turbulent, experiments have shown that finite-size (larger than Kolmogorov scale) bubbles which can deform produce strong drag reduction effects in spite of no recognisable difference in their accumulation near the driving wall [4]. This indicates that the drag reduction induced by finite-size bubbles at very high Reynolds numbers ( $\left.R e \sim 10^{5}-10^{6}\right)$ is fundamentally different from what has been described in the current work. Additionally, in the case of finite-size bubbles, inertial forces become relevant in the deformation dynamics and this makes the Weber number an important parameter. Numerical simulations of such systems would invariably require fully resolved simulations with methods such as front-tracking, volume of fluid, immersed-boundaries etc. 
DEFORMABLE BUBBLES WITH ENHANCED EULER-LAGRANGE TRACKING 


\section{Chapter 5}

\section{Immersed boundary method coupled with interaction potential for deformable interfaces and membranes}

Based on: Vamsi Spandan, Valentina Meschini, Rodolfo Ostilla-Monico, Detlef Lohse, Giorgio Querzoli, Marco D. de Tullio, Roberto Verzicco, 'A parallel interaction potential approach coupled with the immersed boundary method for fully resolved simulations of deformable interfaces and membranes' under review in Journal of Computational Physics, (arXiv id: 1612.03399) 


\subsection{Introduction}

The interaction between fluid flow and an immersed elastic body (fluid or solid) has been studied extensively over the last few decades due to its wide range of applications, for example, bubbles and drops dispersed in a turbulent flow [7, 120], red blood cells flowing through blood vessels [121], pumping motion of ventricles and valves in the heart $[122,123]$, oscillation of large structures such as aircraft wings and high-rise buildings etc. [124]. While the source of elasticity of the immersed body in each of these phenomena is different, the interplay between a deformable body and a surrounding inhomogeneous time dependent flow can result in a complex non-linear system where they determine each other's behaviour in a coupled manner. Additionally, the presence of multiple bodies with different static and dynamic properties interacting with each other gives rise to a wide range of control parameters which makes these systems extremely challenging to study. Over the last few decades, tremendous amount of effort has been devoted to the modelling and simulation of such systems which are often classified in literature as Fluid-Structure Interaction (FSI) problems. Among a variety of techniques developed to tackle FSI problems, the immersed boundary method (IBM) [125-127] has gained immense popularity and has been instrumental in making efficient and accurate simulations of several complex flow systems possible; for example cardiac and vascular hemodynamics [122, 123], suspensions of rigid spheres [128-131], deformable bubbles or drops [132], vehicle aerodynamics $[133,134]$ etc.

One of the biggest advantages of the IBM is that it relies on the use of a single underlying mesh for the fluid flow (hereafter referred to as Eulerian mesh) which does not have to conform/adapt with the moving/deforming immersed body [125-127]. This eliminates the complex and computationally expensive procedure of Eulerian mesh regeneration every time step as the immersed body moves or deforms, resulting in the decoupling of the mesh required for the flow solver from the position and morphology of the immersed body. The surface of the immersed body is discretised independently of the Eulerian mesh and is often called a Lagrangian or a structural mesh. The influence of the immersed body on the flow can be achieved through a volume averaged body force in the fluid governing equations after a careful transfer of information between the Eulerian and Lagrangian meshes. Additionally, the time invariant nature of the Eulerian mesh makes IBM promising for parallelisation on multiple distributed memory computing processors and this has led to breakthroughs in simulations of highly turbulent flows around complex geometries. However, the inclusion of deformability into the immersed boundary framework to study the motion 
of liquid-liquid interfaces or elastic membranes is not straightforward and this is the focus of this chapter.

The emergence of IBM in simulating turbulent multiphase flows began with the seminal work of Uhlmann [135]. He proposed an alternative direct forcing scheme which requires computing the IBM forcing term on Lagrangian markers uniformly distributed over the surface of the immersed body. Several additional features and improvements to this initial idea has led to a massive growth in the use of IBM for dispersed multiphase flows [132, 136-138]. However, the inclusion of deformability into the immersed boundary framework to study the motion of deformable boundaries (liquid-liquid interfaces or elastic membranes) is not straightforward. The approach suggested by Schwarz et al. makes use of spherical harmonics to simulate bubbles or drops of varying shape which is computed through a minimization of the local displacement energy induced by the pressure and surface tension forces [132]. To simulate deformable bodies with several degrees of freedom, de Tullio and Pascazio [139] employ a simple spring network model with an interaction potential where the shape is computed through a minimisation of the local displacement energy induced by the local stress tensor and the spring forces. They demonstrated that numerical simulations of a variety of problems involving large accelerations can be realised using this strongly coupled interaction potential Navier-Stokes IBM solver (e.g. motion of rigid bodies, thin elastic structures, flapping or flexible bodies like flags or leaflets of bio-prosthetic aortic valve etc.). Here it is important to notice that while both the approaches are different for practical purposes, they follow the fundamental principle of minimum potential energy.

In the field of multiphase flows, several techniques have been developed to understand the motion and influence of particles, drops or bubbles in a turbulent flow (e.g. point-particle, Volume of Fluid, level-set, front tracking, Physalis etc.) [5]. When the inherent surface tension forces in the bubbles or drops are not strong enough, they deform according to local flow conditions and also alter them simultaneously. The challenge in direct numerical simulations of such flows arises from the wide range of length, time scales and regimes involved, see [7, 45, 140] for detailed reviews. Numerically handling a sharp boundary between different phases and the singularity of the surface tension term in the governing equations is non-trivial [141]. Additionally, since the density is not uniform in the flow domain, the pressure cannot be computed using fast Poisson solvers, but through relatively slower iterative methods. The complex algorithms and procedures put in place to tackle the above mentioned issues have restricted the scale of multiphase flows that can be studied, for example, state-of-art parallel simulations can only reach up to $O\left(10^{2}\right)$ 
deformable drops/bubbles in a reasonably turbulent flow [7]. In order to scale up numerical simulations of dispersed multiphase flows, there is a need for development of alternative methods which can account for the various active physical mechanisms in the flow.

In this chapter we show the validity and use of a new phenomenological approach to replicate the deformation of closed liquid-liquid interfaces under given flow conditions. One of the big advantages of this approach is the computationally inexpensive nature of the deformation algorithm which allows for simulations of large scale dispersed multiphase flows. The deformability of any immersed drop or bubble is replicated by solving for the dynamics of a three-dimensional spring network spread over its surface. Hereafter we refer to this technique as the interaction potential (IP) model. The Navier-Stokes equations which govern the fluid motion inside and around the immersed body are solved using direct numerical simulations (DNS) while IBM is used to enforce the interfacial boundary conditions (for example no-slip or free-slip). Thus the fluid motion and the effect of an immersed interface on the flow is fully resolved, while the deformability is captured through a stable, versatile, easy to implement and computationally inexpensive IP model. In later sections we will further discuss why the IP model is able to sufficiently replicate the dynamics of deforming liquid-liquid interfaces.

The IBM described in this chapter makes use of the moving-least-squares (MLS) approximation which is crucial when the system involves moving and deforming boundaries. However, the computational cost of MLS increases rapidly with increase in the resolution of the immersed bodies. New algorithms or a parallel implementation of the computation on distributed memory processors thus become an invaluable tool in scaling up fully resolved flows interacting with several moving/deforming immersed bodies. In particular, parallelisation is an attractive prospect given the increasing availability of cost-efficient high performance computing facilities. A parallel implementation of a flow solver involving multiple deforming bodies is not a straightforward task due to many algorithmic complexities. The challenge lies underneath the fact that two different meshes (Eulerian and Lagrangian) are required for the complete solution and different parallelisation strategies would be required to make use of multiple processors effectively.

To understand this, we briefly describe the various steps that are need to be completed on both the Eulerian and Lagrangian mesh to fully simulate a FSI problem: (i) Computing the solution for the fluid phase governing equations on the Eulerian mesh. (ii) Interpolating the Eulerian flow velocity on the Lagrangian markers and computing the required volume averaged IBM forcing term. (iii) Enforcing the 
interfacial boundary condition on the Eulerian mesh through the volume averaged forcing term in the fluid governing equations. (iv) Transferring the local flow conditions onto the Lagrangian mesh followed by transporting and deforming the immersed body. While the Eulerian mesh is fixed in space and time, the Lagrangian mesh can move with multiple degrees of freedom and be distributed over several processors. It is also important to note that unlike the Eulerian mesh, the connectivity of the Lagrangian mesh needs to be stored by all processors. Additionally, the transfer functions built to exchange information between the Eulerian and Lagrangian mesh may require data from neighbouring processors, thus requiring the storage of multiple ghost layers (information from neighbouring processors) in the processor's memory.

Keeping this in mind, in the last part of this chapter we describe a parallelisation scheme designed to track the time evolution of several deformable bodies (e.g. vesicles, drops, biological tissues etc.) immersed in turbulent flows. This strategy is built upon the already underlying parallelisation scheme implemented for the fluid solver, thus reducing the downtime of overall code development. In particular, the benefits of the parallelisation is oriented towards simulation of dispersed phase systems with several thousand deforming drops, bubbles, vesicles or bodies moving in a highly turbulent carrier fluid phase.

In the next section we give an overview of the governing equations for the solution of the fluid phase, implementation of the immersed boundary method using MLS and the interaction potential approach for computing the deformation of elastic bodies. In section 3, we show how the interaction potential approach can be used to study deformation of drops/bubbles where the flow is dynamically coupled with the interface morphology. These results are validated with analytical solutions and experimental measurements taken from literature. In section 4, we discuss the data structures required and also the parallelisation strategy to scale up the problem to study several thousand deforming immersed bodies. Finally, we provide a summary in section 6 .

\subsection{Governing equations and numerical scheme}

For the fluid phase we solve the Navier-Stokes equations governing incompressible flow in a Cartesian box as given in equations 1.1 and 1.2 in $\S 1$. In the following text, velocity and force fields on the Lagrangian and Eulerian meshes are represented by upper case and lower case symbols, respectively.

In equation 1.1 of $\S 1, \mathbf{f}_{\mathbf{b}}$ is the volume averaged force arising from the IBM and is included to enforce the interfacial boundary condition.

We now describe the procedure of constructing the Lagrangian mesh and the 
schemes used to transfer flow quantities between the Lagrangian and Eulerian mesh which is a crucial ingredient in IBM. In figure 5.1, we show a schematic of the Lagrangian meshes used in this study. Any given surface (closed or open) is discretised into triangular elements where each element is composed of three vertices $\left(\mathrm{v}_{1}, \mathrm{v}_{2}, \mathrm{v}_{3}\right)$ which are connected by edges $\left(\mathrm{e}_{1}, \mathrm{e}_{2}, \mathrm{e}_{3}\right)$. Under the condition that the mass is uniformly distributed on the triangular element, the position of the centroid $\left(c_{1}\right)$ of each triangular element is computed based on the co-ordinates of the vertices. Figure 5.1(a) shows a sphere discretised into triangular elements along with a schematic showing the composition of each triangle.

Following the idea of Uhlmann [135] the force required to enforce the interfacial boundary condition is first computed on markers laid out on the Lagrangian mesh and then transferred to the Eulerian mesh. Here, we consider the triangle centroids to be the Lagrangian markers and are responsible for enforcing the interfacial boundary condition. The vertices and edges of the discretised triangular elements play a role in the deformation dynamics and will be explained later.

The next step is to build a transfer function around each Lagrangian marker (here the centroid $c_{i}$ ) which would be used to exchange information between the Eulerian and Lagrangian mesh. As noted previously, we adopt the moving-least-squares (MLS) $[142,143]$ approach which is part of the class of meshless approximations and has been used previously in several fields such as element free Galerkin methods [144148], computer graphics [149-153], and also recently for IBM [137, 139]. In order to compute this transfer function we first need to build a support domain centred around each Lagrangian marker which consists of all Eulerian grid nodes closer than a threshold value in each direction. By taking a threshold value of $r_{i}=1.5 \Delta x_{i}$ in each direction, a three-dimensional support domain consisting of $N_{e}=27(3 \times 3 \times 3)$ Eulerian nodes is built around each Lagrangian marker. In figure 5.1(b) we show a schematic of a two dimensional support domain consisting of nine Eulerian cells around the centroid c1. The next step is to use these Eulerian cells and build a transfer function through which any quantity $q_{i}$ defined on the Eulerian nodes can be interpolated on the Lagrangian marker (i.e centroid of each triangular element). The same transfer function can be used to extrapolate the force computed on the Lagrangian markers $\left(F_{i}\right)$ to the Eulerian mesh $\left(f_{i}\right)$.

The MLS interpolation of $q_{i}$ at a Lagrangian marker $\left(c_{\mathrm{i}}\right)$ is defined as follows.

$$
Q_{i}\left(\boldsymbol{x}^{l}\right)=\boldsymbol{p}^{T}\left(\boldsymbol{x}^{l}\right) \boldsymbol{a}\left(\boldsymbol{x}^{l}\right)=\sum_{j=1}^{m} p_{j}\left(\boldsymbol{x}^{l}\right) a_{j}\left(\boldsymbol{x}^{l}\right)
$$

In equation (6.1), $Q_{i}$ is the quantity interpolated on the Lagrangian marker while 
(a)
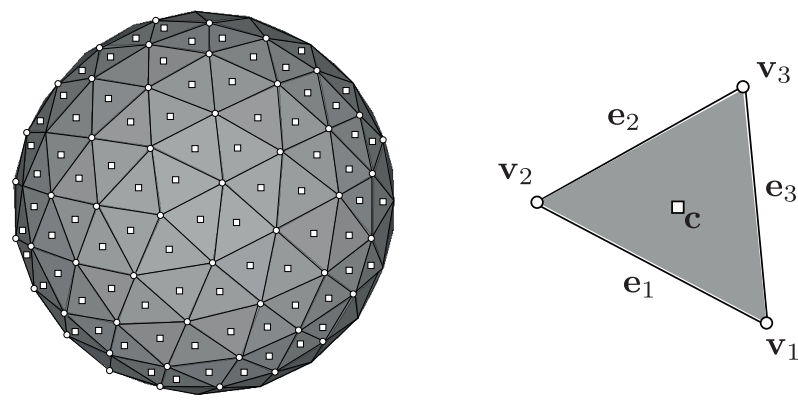

(b)

(c)
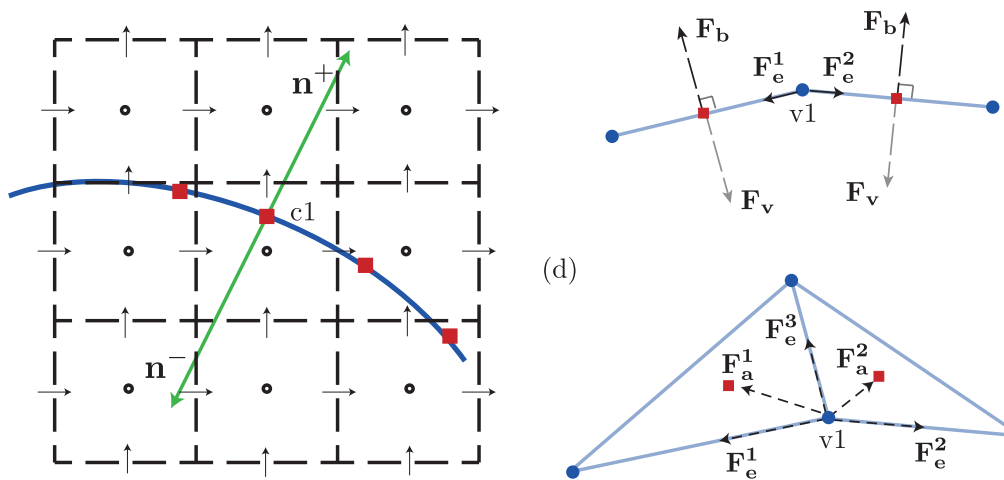

(d)

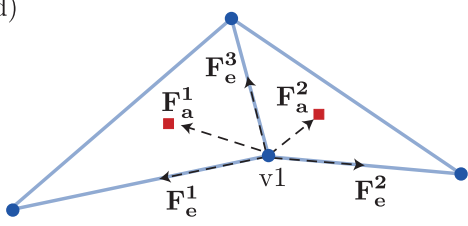

- Lagrangian markers (centriods)

$\longrightarrow$ Position of Eulerian velocities in staggered arrangement

- Edges of triangular elements

$\rightarrow$ Normals to the triangular element with centroid c1

Figure 5.1: Schematic of the Lagrangian mesh (a) A sphere discretised using triangular elements; right panel shows an individual triangular element composed of three vertices, three edges and one centroid. (b) Schematic of a two-dimensional support domain around the centroid c1 (red squares) consisting of nine Eulerian cells; arrows show the position of Eulerian velocities in a staggered formation. (c),(d) Schematic of the direction of the various elastic forces acting on the triangular elements. 
$\boldsymbol{p}^{T}\left(\boldsymbol{x}^{l}\right)$ is a basis function vector with dimension $m . x$ is the position vector of the Lagrangian marker. In this work we consider a linear basis function with $\boldsymbol{p}^{T}\left(\boldsymbol{x}^{l}\right)=$ $[1, x, y, z]$, i.e. $m=4$, which is cost-efficient and also able to represent the gradients in the Eulerian field with second order accuracy. $\boldsymbol{a}\left(\boldsymbol{x}^{l}\right)$ is the vector of coefficients obtained by minimising the weighted L2 norm $J$ as follows:

$$
J=\sum_{k=1}^{N_{e}} W\left(\boldsymbol{x}^{l}-\boldsymbol{x}^{k}\right)\left[\boldsymbol{p}^{T}\left(\boldsymbol{x}^{k}\right) \boldsymbol{a}\left(\boldsymbol{x}^{l}\right)-q_{i}^{k}\right]^{2}
$$

Here $W\left(\boldsymbol{x}^{l}-\boldsymbol{x}^{k}\right)$ is a weight function; we use the exponential weight function which is given as follows.

$$
W\left(\boldsymbol{x}^{l}-\boldsymbol{x}^{k}\right)= \begin{cases}e^{-\left(r_{k} / \alpha\right)^{2}}, & r_{k} \leq 1 \\ 0, & r_{k}>1\end{cases}
$$

where $\alpha$ is a constant of shape parameter and $r_{k}$ is given by

$$
r_{k}=\frac{\left|x^{L}-x^{k}\right|}{r_{i}}
$$

$r_{i}$ is the size of the support domain in the $i^{\text {th }}$ direction as defined previously. Other commonly used shape functions are the cubic spline and quadratic spline functions and a spline function with any order of continuity can be constructed using the steps detailed in Liu and Gao [143]. Minimising $J$ in equation (6.2) leads to $\boldsymbol{A}\left(\boldsymbol{x}^{l}\right) \boldsymbol{a}\left(\boldsymbol{x}^{l}\right)=$ $\boldsymbol{B}\left(\boldsymbol{x}^{l}\right) \boldsymbol{q}_{i}^{k}$ where

$$
\begin{gathered}
\boldsymbol{A}\left(\boldsymbol{x}^{l}\right)=\sum_{k=1}^{N_{e}} W\left(\boldsymbol{x}^{l}-\boldsymbol{x}^{k}\right) \boldsymbol{p}\left(\boldsymbol{x}^{k}\right) \boldsymbol{p}^{T}\left(\boldsymbol{x}^{k}\right) \\
\boldsymbol{B}\left(\boldsymbol{x}^{l}\right)=\left[W\left(\boldsymbol{x}^{l}-\boldsymbol{x}^{1}\right) \boldsymbol{p}^{T}\left(\boldsymbol{x}^{1}\right) \ldots W\left(\boldsymbol{x}^{l}-\boldsymbol{x}^{N_{e}}\right) \boldsymbol{p}^{T}\left(\boldsymbol{x}^{N_{e}}\right)\right] \\
\boldsymbol{q}_{i}=\left[q_{i}^{1} \ldots q_{i}^{N_{e}}\right]^{T}
\end{gathered}
$$

Combining all the above equations, the interpolated quantity $Q_{i}$ can be expressed as follows.

$$
Q_{i}\left(\boldsymbol{x}^{l}\right)=\boldsymbol{\phi}^{T}\left(\boldsymbol{x}^{l}\right) \boldsymbol{q}_{i}=\sum_{k=1}^{N_{e}} \phi_{k}^{l}\left(\boldsymbol{x}^{l}\right) q_{i}^{k}
$$

$\boldsymbol{\phi}^{T}\left(\boldsymbol{x}^{l}\right)=\boldsymbol{p}^{T}\left(\boldsymbol{x}^{l}\right) \boldsymbol{A}^{-1}\left(\boldsymbol{x}^{l}\right) B\left(\boldsymbol{x}^{l}\right)$ is the transfer function containing the shape function coefficients for each Lagrangian marker. This shape function is used to interpolate the value of the intermediate Eulerian velocity $\hat{u}_{i}$ at the exact location of all Lagrangian 
markers and the volume force in each direction is calculated as $F_{i}=\left(V_{i}^{b}-U_{i}\right) / \Delta t$, where $V_{i}^{b}$ is the desired velocity boundary condition on the Lagrangian marker $\left(c_{\mathrm{i}}\right)$ and $U_{i}$ is the Eulerian flow velocity interpolated on the Lagrangian marker using MLS. Under the assumption of a no-slip boundary condition on the interface, the desired velocity $V_{i}^{b}$ is equal to the velocity of the corresponding centroid. This force needs to be transferred back to the Eulerian mesh using the same transfer function built for interpolation in equation (5.8) under the constraint that the total force is conserved during the extrapolation. The force to be included in the Eulerian mesh is written as $f_{b, i}^{k}=\sum_{k=1}^{N_{L}} c_{l} \phi_{k}^{L} F_{i}^{L}$, where $N_{L}$ is the number of Lagrangian markers associated with a Eulerian point $k . c_{l}$ is a scaling factor obtained by imposing the condition that there is no net-gain/loss in the IBM force while transferring flow information from the Lagrangian mesh to the Eulerian mesh which results in the following.

$$
c_{l}=\frac{\Delta V^{l}}{\sum_{k=1}^{N_{e}} \phi_{k}^{l} \Delta V^{K}}
$$

$\Delta V^{L}$ is the forcing volume associated with each Lagrangian marker and is computed as $\Delta V^{l}=A_{l} h_{l} . A_{l}$ is the area of the triangular element associated with the Lagrangian marker (area composed by $\mathrm{v}_{1}, \mathrm{v}_{2}, \mathrm{v}_{3}$ in figure 5.1) and $h_{l}=1 / 3 \sum_{k=1}^{N_{e}} \phi_{k}^{L}\left(\Delta x^{k}+\right.$ $\left.\Delta y^{k}+\Delta z^{k}\right) . \quad \Delta V^{k}$ is the volume of the Eulerian cell $k$ involved in the support domain. Here it is important to note that the transfer functions built using this approach conserves momentum on both uniform and stretched grids while reasonable accuracy is retained for torque equivalence on slightly stretched grids [137, 139]. For example, Vanella and Balaras [137] report that with $10 \%$ grid stretching, the net loss/gain in torque conservation is less than $0.5 \%$.

The calculation of hydrodynamic forces (pressure and viscous stresses) acting on the surface of any dispersed body in an IBM simulation is not straightforward as the Lagrangian and the Eulerian meshes do not necessarily align with each other at a given time instant. Since the surface of the dispersed bodies are discretised using triangular elements, the local pressure and viscous forces are first computed on the Lagrangian markers (centroids of triangular elements); the total external force on a triangular element with area $A_{l}$ and surface normal $\boldsymbol{n}_{l}$ is calculated as $\boldsymbol{F}_{\text {ext }}^{l}=\left(-p_{l} \boldsymbol{n}_{l}+\boldsymbol{\tau}_{l} \cdot \boldsymbol{n}_{l}\right) A_{l}$. To evaluate $p_{l}$ and $\tau_{l}$, which are the pressure and viscous forces acting on a triangular element $l$, respectively a probe is sent along the normal of each triangular element with its centroid as the origin. The length of the probe $h_{l}$ is equal to the mean local grid size and the MLS interpolation described above is used to interpolate both pressure and velocity gradients at the end point of the probe. The velocity gradients can also be computed from the derivatives of the shape functions [143]. The value of 
the pressure gradient along this normal is computed from the momentum equation normal to the triangular element which gives $\frac{d p}{d n}=-\frac{D \boldsymbol{U}_{L}}{d t} \cdot \boldsymbol{n}_{L}$. The pressure on the Lagrangian marker (centroid) is then computed as follows:

$$
p_{l}=p_{l}^{*}+h_{l} \frac{D \boldsymbol{U}_{l}}{D t} \cdot \boldsymbol{n}_{l}
$$

$p_{l}^{*}$ is the pressure at the probe endpoint which is estimated through a MLS approximation and $\frac{D \boldsymbol{U}_{l}}{D t}$ is the acceleration of the Lagrangian marker [137, 139, 154]. The shear stress $\boldsymbol{\tau}_{l}$ on the Lagrangian marker is computed based on the velocity gradients interpolated at the probe endpoint. This holds true under the assumption that the velocity of the fluid near the surface of the body varies linearly. An important note here is that when the nature of the immersed body is such that fluid loads on either side of the interface are relevant, the pressure and viscous forces need to computed on both sides of the surface thus requiring two probes sent along the normal to every triangular element, one each along the positive and negative normal, respectively i.e. $\boldsymbol{F}_{\text {ext }}^{l}=\left[\left(-\left(p_{l}^{+}-p_{l}^{-}\right) \boldsymbol{n}_{l}^{+}+\left(\boldsymbol{\tau}_{l}^{+}-\boldsymbol{\tau}_{l}^{-}\right) \cdot \boldsymbol{n}_{l}^{+}\right)\right] A_{l}$; the subscripts ${ }^{+}$and - represent quantities evaluated on the end points of the probe on either side of the surface [137, 139, 154].

\subsubsection{Interaction potential approach for deformation}

As mentioned in section 1, the dynamics of deformation is computed based on a minimum energy concept which we describe here briefly. The surface of any immersed body is first discretised using triangular elements (c.f. figure 5.1) the edges of which are composed of hypothetical linear/non-linear springs thus resulting in a two-dimensional network of springs. Under the influence of external forces such as pressure fluctuations or viscous stresses, the spring network undergoes deformation thus storing potential energy into the system. The potential is converted to nodal forces acting on individual triangular vertices by differentiating the potentials with respect to its corresponding displacement. The force acting on each triangular vertex is converted into an acceleration and integrated based on Newton's second law of motion and the position of each individual vertex is then updated accordingly.

The first form of potential is the in-plane elastic potential $\left(W_{e}\right)$ which comes from the work done by an external force parallel to the plane of a triangular face and is converted into elastic energy stored into every spring connecting the triangle. We also consider an out-of-plane deformation $\left(W_{b}\right)$ for which the total potential is computed based on a bending spring connecting the centroids of two adjacent triangular faces. This out-of-plane bending potential is stored in a pair of two faces sharing an edge 
and is a function of the angle between the normals of the respective faces. Additional potentials can be included which constrain the geometrical properties of the overall immersed body. For example, we can include a volume or area potential $\left(W_{v}\right.$ or $\left.W_{a}\right)$ which is a function of the change in volume/area of a single element with respect to an initial reference state. All the individual potentials are formed as given in the equations below.

$$
\begin{gathered}
W_{e}=\frac{1}{2} k_{e} x^{2} \\
W_{b}=k_{b}(1-\cos \theta) \\
W_{v}=\frac{1}{2} k_{v}\left(\frac{V-V_{0}}{V_{0}}\right)^{2} V_{0} \\
W_{a}=\frac{1}{2} k_{a}\left(\frac{A-A_{0}}{A_{0}}\right)^{2} A_{0}
\end{gathered}
$$

In the above equations, $k_{e}, k_{b}, k_{v}$, and $k_{a}$ are the elastic constants for in-plane deformation, out-of-plane deformation, volume constraint and area constraint potentials, respectively. $\boldsymbol{x}$ is the change in length of a single edge; $\theta$ is the angle between the normals of two triangular faces sharing an edge; $V_{0}, V$ and $A_{0}, A$ are the corresponding initial (reference) and deformed volumes and areas of each triangular element, respectively. While the equations (5.11)-(5.14) can be used to simulate homogeneous isotropic materials, the interaction potential approach can also used for inhomogeneous anisotropic materials by changing the functional form of the elastic potentials [139].

The total potential energy of the system is then represented as a sum of several potentials as given in equation (5.15).

$$
\Pi=W_{e}+W_{b}+W_{v}+W_{a}
$$

In figure 5.1(c), (d) we show a 2D and 3D schematic, respectively of the forces originating from these potentials on the vertices of the triangular elements. $\boldsymbol{F}_{e}$ is the in-plane elastic force and it acts along the edges connecting two vertices; $\boldsymbol{F}_{b}$ and $\boldsymbol{F}_{v}$ are the out of plane bending and volume constraint forces which act along the normal at the centroids of each triangular element. $\boldsymbol{F}_{a}$ is the force originating from the area constraint potential and is directed towards the centroid of the triangular element. Once the forces on each of the triangular vertices are known, individual nodes are moved based on the equation $m \ddot{\boldsymbol{x}}^{\mathrm{v}_{i}}=F_{\mathrm{ext}}^{\mathrm{v}_{i}}+F_{\mathrm{int}}^{\mathrm{v}_{i}} ; F_{\mathrm{ext}}^{\mathrm{v}_{i}}$ and $F_{\text {int }}^{\mathrm{v}_{i}}$ are the external and 
internal forces acting on the triangular node $\mathrm{v}_{i}, \ddot{\boldsymbol{x}}$ and $m$ are the acceleration and mass of individual nodes. In the previous section $F_{\text {ext }}$ was calculated on the centroid of each triangle. This force is transferred to an individual triangular vertex as $F_{\mathrm{ext}}^{\mathrm{v}_{i}}=$ $\sum_{j=1}^{\mathrm{n}_{f i}}(1 / 3) F_{\text {ext }}^{\mathrm{c}_{j}}$; where $\mathrm{n}_{f i}$ is the number of faces each vertex is connected to and $F_{\text {ext }}^{\mathrm{c}_{j}}$ is the external force computed on the triangle centroid (Lagrangian marker) $\mathrm{c}_{j}$. The calculation of $m$, which is the mass of individual triangular node is straightforward for surfaces made of materials where the density and thickness is known a priori. In cases where the immersed bodies are drops or bubbles, calculating $m$ of the triangular nodes becomes tricky as there is no physical definition of the density and thickness of a liquid-liquid interface. In such a case $m$ of the triangular nodes becomes a free parameter and to overcome this we fix the value of $m=1$ and then correspondingly tune the elastic constants. This is detailed more in the next section.

Computing the individual potentials according to equations (5.11)-(5.14) in the interaction potential approach requires selection of several parameters $\left(k_{e}, k_{b}, k_{v}, k_{a}\right)$. Once again, this step is straightforward for membranes where the elastic moduli are already known [139]. Additionally, it is important to note that when the surface is discretised with non-uniform triangles such that the lengths of the edges of triangle vary, $k_{e}$ should be computed based on the model proposed by van Gelder [155]. This is to ensure that at the rest state of the elastic structure the properties of all the springs connecting the vertices are equivalent. On the other hand, simulating liquid-liquid interfaces using the interaction potential approach would require the use of ad-hoc elastic constants as again there is no direct physical correlation between the elastic constants and the surface properties of a liquid-liquid interface. The procedure of estimating these ad-hoc elastic constants will be described in detail in the next section.

Here it is important to note that modelling an elastic membrane or an interface using the interaction potential approach is a discrete formulation of the elasticity governing equations and thus a simplification of the existing continuum models. It has been shown in previous studies that through a careful design of the spring network and the selection of appropriate elastic constants the mechanics of several elastic membranes can be exactly reproduced $[139,156,157]$. While such a formulation is useful and necessary for complex simulations of several immersed deformable bodies owing to its simplicity and lower computational cost, it has its limitations. In particular for liquid-liquid interfaces, when the drops/bubbles deform to such an extent that they approach the critical Capillary/Weber number for breakup, the Lagrangian resolutions become terminally high. Such scenarios are better handled with techniques such as VOF or level-set. When the Capillary/Weber numbers for the drops/bubbles are well below their breakup limit, the IP model provides a 
viable alternative to simulate large scale dispersed multiphase flows with realistic computational expenses. Here we would like to note that the derivation of the interaction potential approach is not unique to this work and variants of this method have already been used previously to predominantly study red blood cells [156-159]. In this chapter we show how this approach can also be extended for more complex cases such as large scale flows with dispersed deforming drops/bubbles.

Another important issue in the simulations involving FSI problems is the type of coupling used i.e. loose (explicit) versus strong (implicit). In the loosely coupled (explicit) case, the fluid and the immersed body governing equations are solved separately one after the another with a transfer of information between them every time step. On the other hand, in the strongly coupled (implicit) case the governing equations are solved in an iterative manner for each time step using a predictor corrector scheme until sufficient convergence is achieved. A detailed solution procedure for a strongly coupled IBM-FSI Navier Stokes solver with the provision for the interaction potential approach is given in de Tullio and Pascazio [139] where the governing equations are solved using a Hammingś fourth order predictor-corrector scheme. In our code, we have provisions for both a strong (implicit) and weak (explicit) coupling between the fluid and the immersed body. For the simulations shown in the following sections, loose coupling is used given its computationally inexpensive nature. Also it has to be remembered that strong coupling is only needed when added mass effects from the immersed body become important and the recent work of Schwarz et al. [138] gives insights into how this can be tackled smartly while there is still loose coupling between the fluid and the immersed body. We now move on to combining DNS of the fluid governing equations along with a moving least squares IBM coupled with the interaction potential approach to simulate deformable drops/bubbles and heart ventricles/valves.

\subsection{Liquid-liquid interface dynamics using the potential approach}

In order to replicate the deformation dynamics of drops or bubbles using the IP model, we first need to devise a method to compute the elastic constants of a given spring network which can represent a liquid-liquid interface with a given surface tension. As mentioned earlier, this is not straight-forward since there is no direct physical correlation between the elastic constants and the surface tension of a liquid-liquid interface. Here we use a reverse-engineered approach and perform a single simulation with a set of intuitively chosen elastic constants and estimate the 
surface tension of the immersed drop by comparing its morphology with previously known analytical solutions. By using the same set of elastic constants but for different flow conditions we also show that such an approach is self-consistent and reliable. Our goal here is to show that the IP model for deformation can be used to replicate the deformation dynamics of liquid-liquid interfaces under given flow conditions.

Before we analyse the results, it important to understand from a theoretical point of view why the IP model can be expected to mimic the deformation behaviour of drops/bubbles. When an initially spherical drop is immersed in a fluctuating flow field, the viscous and pressure stresses tend to deform the drop while the surface tension forces tend to resist the deformation and bring it back to its original spherical shape. During deformation, the total potential energy of the deformation stored in the drops surface is given by $W=\int\left[\left(p_{i}-p_{o}\right)-0.5 \gamma\left(1 / R_{1}+1 / R_{2}\right)\right] d n \cdot d A$, here $p_{i}, p_{o}$ are the pressures inside and outside the drop, $\gamma$ is the surface tension while $R_{1}, R_{2}$ are the principal radii of curvature [160]. Under the action of external forces, the shape of the drop adjusts itself such that the total displacement potential energy $W$ tends to a minimum. The shape of the deformed drop can be computed by parametrising the surface in such a manner that it satisfies the above condition under certain constraints (for example an ellipsoidal shape or more complex shapes using spherical harmonics) $[132,161]$. The IP model is mathematically analogous in a way that the forces on each triangular vertex (c.f. figure 5.1(c),(d)) act such that the total potential energy of deformation $\Pi=W_{e}+W_{b}+W_{v}+W_{a}$ tends to a minimum. Although the IP model for deforming drops is not an exact representation of the surface tension phenomena it can be considered as a phenomenological approach which can mimic the drop deformation characteristics given the fact that both the exact representation and the IP model rely on the fundamental principle of minimum potential energy.

\subsubsection{Deformation of a neutrally buoyant drop in shear flow}

For the first test case we choose a neutrally buoyant drop deforming in a laminar shear flow which has a simple configuration and a limited set of control parameters. Variants of this problem have been studied for a long time and several analytical and phenomenological models already exist in literature which can accurately predict the deformation dynamics of the immersed drop [58, 59, 68]. For this simulation, we use a Cartesian box which is wall-bounded in the vertical direction $\left(\hat{e}_{z}\right)$ and fully periodic in the horizontal directions. The top and bottom walls move in the opposite direction parallel to each other with the same velocity to generate a laminar shear in the domain. A triangulated sphere as shown in figure 5.1(a) is positioned in the flow at a distance $0.5 L_{z}$ from the walls ( $L_{z}$ is the gap between the walls). 
The degree of deformation and orientation of a viscous drop in the presence of a velocity gradient depends on the Capillary number $C a=\mu_{f} R \dot{\gamma} / \sigma$, where $R, \dot{\gamma}$, $\sigma$ and $\mu_{f}$ are the drop radius, local strain rate, surface tension and fluid viscosity, respectively. For these simulations the viscosity ratio of the droplet and the carrier phase is set to 1 , i.e. $\hat{\mu}=\mu_{d} / \mu_{c}=1$. Since this simulation will be used to 'tune' the elastic constants to replicate a liquid-liquid interface, we assume that the immersed drop is very small in comparison to the distance between the walls and the immersed boundary forcing $\boldsymbol{f}$ in equation 1.1 is set to zero. This is done so that the immersed sphere only experiences the forces generated due to the laminar shear from the moving walls and not due to any wall effects. It also facilitates in quickly tuning the elastic constants due to the ease in setting up such a simulation. Here it is important to point out that while we choose the system of a neutrally buoyant deforming drop any other flow with known solutions can be used.

To estimate the ad-hoc surface tension value for any given spring network the following steps are undertaken. We first fix the Lagrangian resolution i.e the number of vertices on the surface of a sphere and initialize a spherical drop under a given shear rate $\dot{\gamma}$ with a set of elastic constants. For the first set of elastic constants, $k_{e}$ and $k_{a}$ are fixed to large values in comparison to $k_{b}$ thus resulting in an extremely stiff drop. $k_{v}$ is chosen to be much larger than the rest of all constants as this ensures incompressibility of the immersed drop. Once the first set of elastic constants are chosen the drop is allowed to deform under the action of the velocity gradient $\dot{\gamma}$ according to the potential approach described in the previous section. If the final state of the drop is close to spherical, the elastic constant $k_{e}$ and area constant $k_{a}$ are reduced simultaneously which reduces the overall stiffness resulting in deformation of the spring network. Here it is important to note that if both $k_{v}$ and $k_{a}$ are fixed to a large value, which would imply conservation of both the volume and total area, the triangulated sphere would represent a vesicle. To represent a drop, both $k_{e}$ and $k_{a}$ are reduced to a low enough value such that the drop deforms approximately into an ellipsoid as shown in figures 5.2(a), (b). The initial and final states of the triangulated sphere shown in figure 5.2(a),(b) are for two different Lagrangian resolutions i.e the spheres are discretised using 320 and 1280 triangular elements (faces), respectively.

We now give an estimate of the elastic constants used for the following analysis. For any given spring-network with a prescribed elastic modulus and mass distribution, the elastic constant for a given edge of length $l$ is proportional to Young's modulus for the material, thickness of the membrane (here interface), area of the triangles sharing the edge and inversely proportional to the square of the length of the edge [155]. Under the approximation that the sphere is discretised in such a way that 
all the faces have roughly the same area, the ratio $k_{e} l_{e}^{2}\left(l_{e}\right.$ is the mean edge length on the sphere) should be independent of the number of triangular elements . For the sphere shown in figure $5.2 \mathrm{a}, k_{e} l_{e}^{2}=3 \times 10^{-3}$ and $l_{e} / L=1.5 \times 10^{-2}$. The bending constant is set as $k_{b}=k_{e} / 10.0$ while the volume constant is set to a very large value; in this case $k_{v}=\alpha_{v} k_{e}$ where typically $\alpha_{v} \sim 10^{4}-10^{5}$ is sufficient to ensure that incompressibility of the sphere is ensured. The area constant $k_{a}$ is set equal to that of the elastic constant. As discussed previously, when simulating other systems (for example vesicles) which also require surface area conservation along with volume conservation the area constant $k_{a}$ is also set to relatively large value similar to the volume constant $k_{v}$. On changing the Lagrangian resolution the elastic constants should also be scaled in a way that the product $k_{e} l_{e}^{2}$ remains unchanged. This ensures that the physical properties of the spring network remains similar and is independent of the resolution used. The time step for the following simulation is set to $\Delta t=10^{-4} / \dot{\gamma}$ ( $\dot{\gamma}$ is the imposed strain rate) and the convergence criteria is such that the change in the total surface area of the sphere in successive time steps is less than $10^{-6}$.

Once the deformation of the sphere has reached a steady state under a laminar shear flow, we compute its semi-major axis $(L)$ and semi-minor axis $(B)$. Next, we use a phenomenological model proposed by Maffettone and Minale [68] (hereafter called 'MM' model) to estimate the Capillary number $\mathrm{Ca}$ for which a neutrally buoyant immersed drop would have the same final state under similar flow conditions. The model proposed by MM [68] predicts the deformation of a drop in an arbitrary velocity field under the assumption that the drop is ellipsoidal in shape. For a simple flow field such as a laminar shear flow the model can be analytically solved to give the steady state values of the semi-major $(L)$ and semi-minor axis $(B)$ of the deformed drop as given below.

$$
\begin{aligned}
& L^{2}=\frac{f_{1}^{2}+C a^{2}+f_{2} C a \sqrt{f_{1}^{2}+C a^{2}}}{\left(f_{1}^{2}+C a^{2}\right)^{1 / 3}\left(f_{1}^{2}+C a^{2}-f_{2}^{2} C a^{2}\right)^{2 / 3}} \\
& B^{2}=\frac{f_{1}^{2}+C a^{2}-f_{2} C a \sqrt{f_{1}^{2}+C a^{2}}}{\left(f_{1}^{2}+C a^{2}\right)^{1 / 3}\left(f_{1}^{2}+C a^{2}-f_{2}^{2} C a^{2}\right)^{2 / 3}}
\end{aligned}
$$

In equations (5.16) and (5.17), $f_{1}$ and $f_{2}$ are coefficients which depend on the viscosity ratio $(\hat{\mu})$ and $C a$ is the Capillary number.

$$
f_{1}=\frac{40(\hat{\mu}+1)}{(2 \hat{\mu}+3)(19 \hat{\mu}+16)} \quad f_{2}=\frac{5}{2 \hat{\mu}+3},
$$

This model has already been used in other studies and has been described in $\S 2$ and $\S 3$; for example to predict hemolysis of red blood cells [71] and also deformation and 
(a)
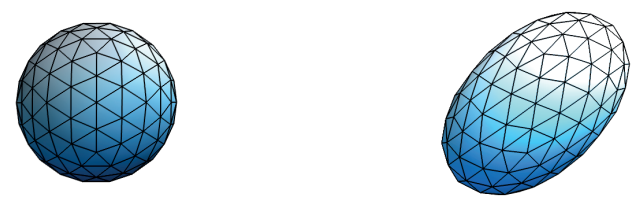

(b)
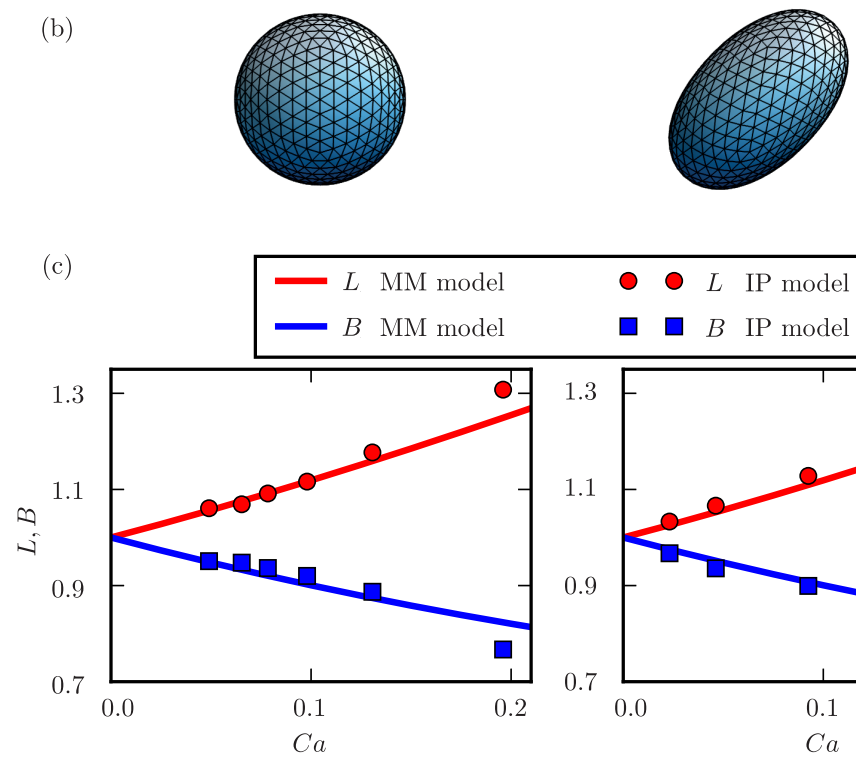
$\bigcirc \bigcirc L \quad$ IP model
$\square \quad$ 口 $B$ IP model

(d)
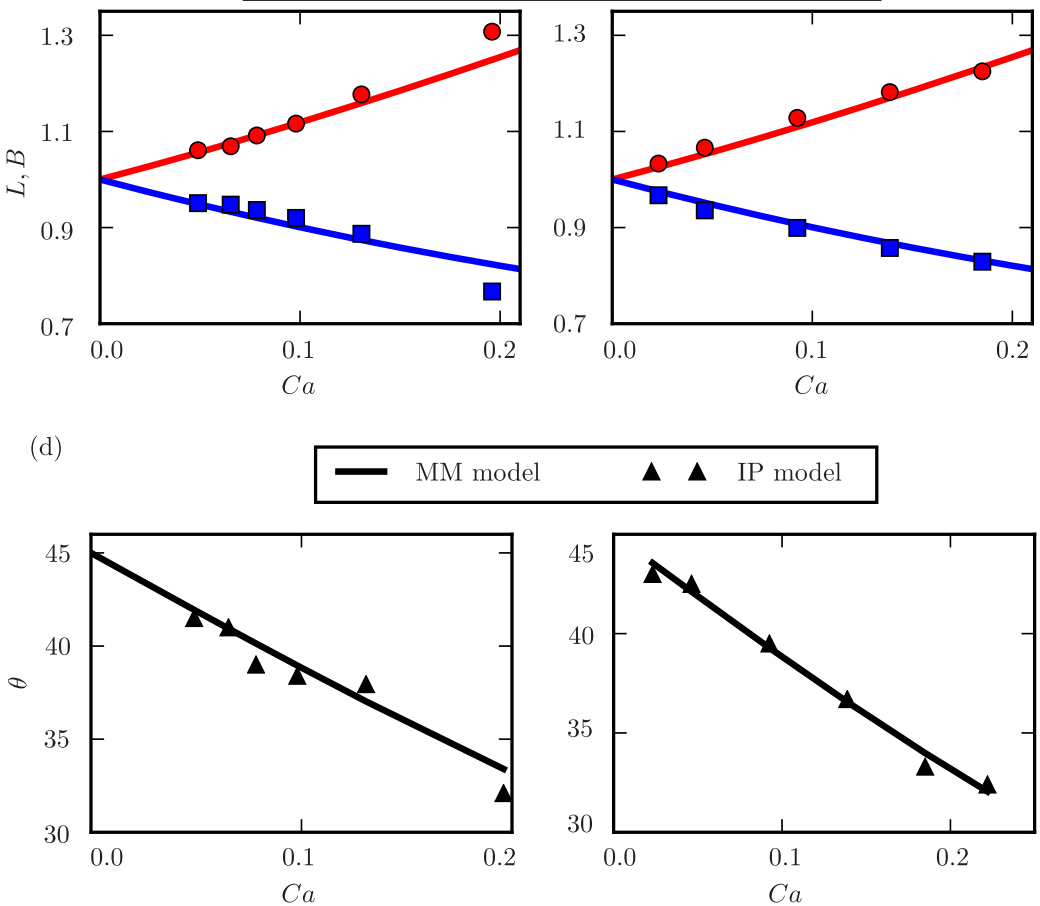

$\Delta \quad \boldsymbol{\Delta}$ IP model

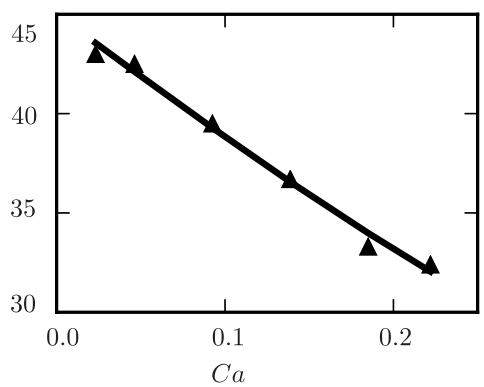

Figure 5.2: Deformation of a neutrally buoyant drop in a laminar shear flow using the IP model. Lagrangian resolution of (a) $N_{\text {faces }}=320$, (b) $N_{\text {faces }}=1280$. In both cases, the viscosity ratio is set to $\hat{\mu}=1$ (c) Comparison of the semi-axes lengths versus the Capillary number (d) Comparison of the angle formed by the major-axis in the shear plane with the velocity direction. 
orientation statistics of drops in turbulent flows [67, 108]. Additionally, experimental studies have shown that under moderate deformations the steady-state droplet shape can be very well described by an ellipsoid [80, 162].

In figure 5.2(c), (d) we plot the analytical solutions (MM model - solid lines) in the form of the lengths of the semi-axes and the orientation angle of the major axis (corresponding to the axes with length $L$ ) with the stream-wise direction versus the Capillary number. Using this as a reference, we check the position of overlap of the semi-axes lengths computed through the IP model with the MM model to estimate the corresponding Capillary number. This match is shown through a vertical dotted line in figure 5.2(c) and since the flow configuration such as drop radius, viscosity and shear rate are already fixed in the simulation, this Capillary number can be directly used to estimate the ad-hoc surface tension value for the chosen elastic constants. The left and right panels in figure 5.2 correspond to different Lagrangian resolutions. The important point to observe here is the reasonably good match between the semi-axes lengths computed from the IP model and the MM model. Small differences in the semi-axes lengths could arise due to multiple reasons, (i) lack of sufficient Lagrangian resolution since in the IP model the surface of the sphere is discretised using markers (ii) MM model assumes a perfectly shaped sphere which deforms into an ellipsoid while the IP model has no constraint of deforming into an ellipsoid (iii) the elastic constants would need further tuning.

Next, we keep the elastic constants the same and change the Capillary number which can be done by either changing the shear rate or the viscosity of the fluid. As shown in figure $5.2(\mathrm{c}, \mathrm{d})$ again the semi-axes lengths computed from the IP model agree reasonably well with the analytical solutions from the MM model. This shows that the ad-hoc surface tension computed by fitting the results from a single simulation using the IP model with MM model is reliable to extend the approach to other flow conditions. A good agreement with the MM model is found also for the orientation angle of the semi-major axes as shown in figure 5.2(d). At higher Capillary numbers $(C a=0.2)$ there is some difference found in the lengths computed from the IP model as compared to MM model (left panel of figure 5.2(c)). However, this is just an effect of the Lagrangian resolution and can be corrected by increasing the number of vertices on the surface of the sphere as is seen in the right panel of $5.2(\mathrm{~d})$.

\subsubsection{Dynamics of a liquid-liquid interface deforming in cross-flow}

In the previous subsection we demonstrated that by tuning the elastic constants for a single flow configuration to compute an ad-hoc surface tension, the IP model can 


$$
\hat{e}_{y} \underset{\hat{e}_{z}}{\longrightarrow} \hat{e}_{x}
$$
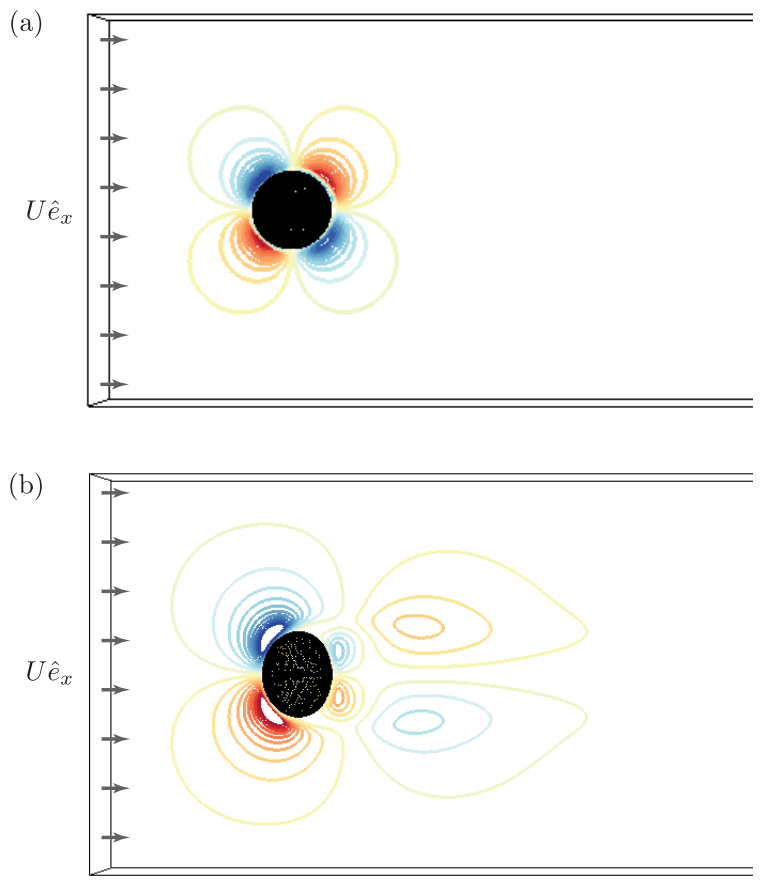
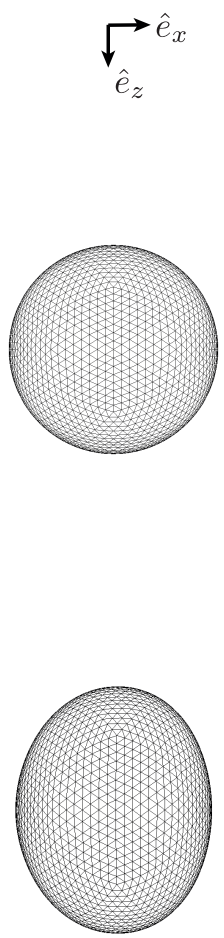

Figure 5.3: Left panels in both (a) and (b) show the contours of velocity in $\hat{e}_{z}$ direction along with the deforming drop at two different time instants. Right panels show the corresponding drop in the form of the deformed triangulated spring network. The Reynolds number of the flow based on the initial drop diameter is set to $R e=150$, while the elastic constants chosen correspond to a Weber number $W e=2$. 


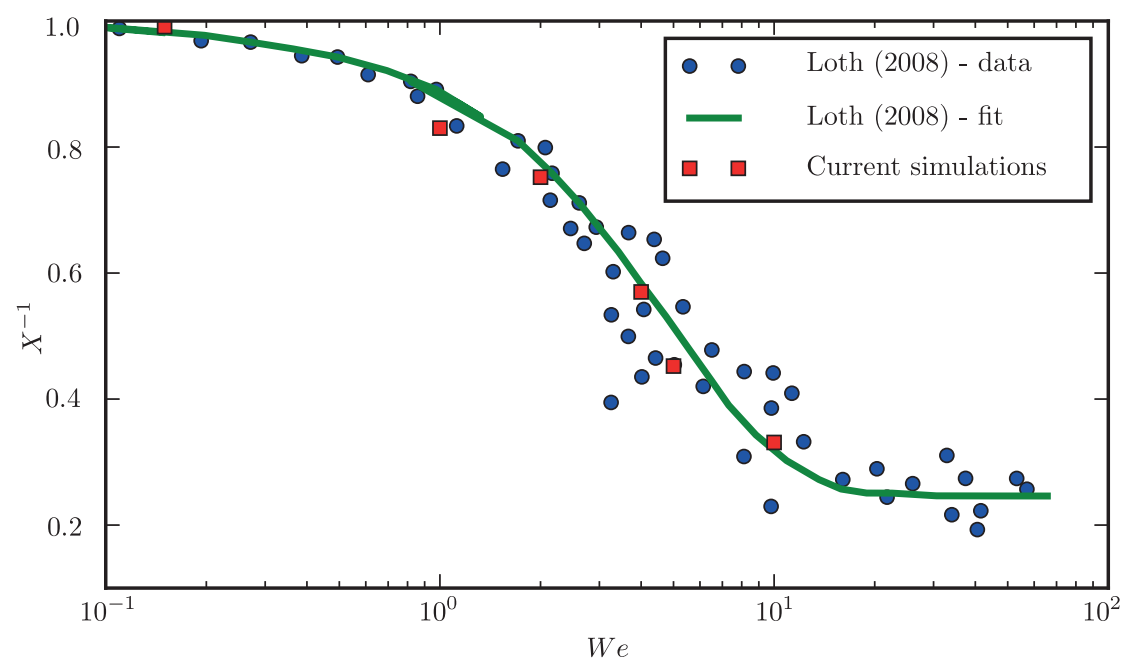

Figure 5.4: Comparison of the inverse aspect ratio $\left(X^{-1}\right)$ of the deformed drop versus Weber number at $R e=150$ with data from Loth [163] for contaminated drops or bubbles

be used to reliably simulate a neutrally buoyant drop deforming in a laminar shear flow. We now move on to simulating a more dynamic problem where the interface is strongly linked to the local flow conditions. In order to do this we take the same test case as done by Schwarz et al. [132] and have a drop immersed in a cross flow and compute the mean shape arising from the resulting flow conditions. The elastic constants are estimated according to the procedure described in the previous section. For such a flow, the aspect ratio of the deforming drop depends strongly on the Weber number $W e=\rho_{f} U_{\text {ref }}^{2} d_{\text {eq }} / \sigma$, which is the ratio of inertia forces acting on the drop in comparison the surface tension forces (for more details see the review by Loth [163]). The cross-stream set up in the domain is influenced by the interface of the spherical drop leading to the development of a boundary layer on the drop surface and a corresponding wake.

The computational domain is taken to be of size $L=(10,5,5) d_{\mathrm{eq}}, d_{\mathrm{eq}}$ is the diameter of the drop in its initial spherical shape. The spherical drop is triangulated with $N_{v}=2562$ nodes and is placed at $\boldsymbol{x}=(0.5,0.5,0.5) L_{z}$. The vertical direction $\left(\hat{e}_{z}\right)$ is wall bounded with stationary free-slip walls; $\hat{e}_{y}$ direction is periodic in nature and a uniform flow of $\boldsymbol{U}=U \hat{e}_{x}$ is imposed in the $\hat{e}_{x}$ direction.

The control parameters for such a problem are the Reynolds number, Re $=$ 
$U d_{\mathrm{eq}} / \nu_{f}$ and the Weber number, $W e=\rho_{f} U_{\mathrm{ref}}^{2} d_{\mathrm{eq}} / \sigma$. The response of the system can be measured through the quantification of the wake of the drop and also through the morphology of the drop. The combined action of the dynamic pressure acting on the faces of the drop and the shear stresses generated from the boundary layer development on the surface of the drop leads to its deformation. In figure 5.3 we show the wall-normal component of the velocity field $\left(u_{z}\right)$ and the corresponding deformed drop represented through the triangulated spring network. The two snapshots shown in figure 5.3 are at two different instants showing the starting up phase and the deforming phase. To quantify the shape of the immersed drop we compute the mean aspect ratio of the bubble measured as the ratio of the lengths of the drop bounding box in the wall-normal and stream-wise directions i.e. $X=l_{z} / l_{x}$, where $X$ is the aspect ratio and $l_{z}, l_{x}$ are the lengths of the box surrounding the deformed drop in the $\hat{e}_{z}, \hat{e}_{x}$ directions, respectively. In figure 5.4 we plot the inverse of the measured aspect ratios of the deformed drop versus the corresponding Weber number and compare it with experimental data from multiple measurements [163]. For these simulations the Reynolds number is fixed to $R e=150$ and the Weber number is changed by modifying the elastic constants for each simulation. A very good match is found between the aspect ratios computed from the IP model and the several experimental measurements of drop shapes found in literature. These simulations further show that the IP model can be reliably used to simulate deformation in liquid-liquid interfaces under given flow conditions.

\subsection{Data structures and pseudo code for Lagrangian mesh parallelisation}

In this section we describe the parallelisation strategy implemented and the data structures required for the parallelising the algorithms described in previous sections. For dealing with a suspension of spherical particles, Uhlmann [164] proposed a 'master' and 'slave' strategy, where each particle is allocated an individual 'master' processor which is responsible for all the computations related to it. Additional 'slave' processors may be allocated to help the 'master' processor. Wang et al. [165] employ a 'gathering-scattering' strategy where a single master processor is responsible for the computation of the Lagrangian force on the immersed bodies and advecting them and this information is scattered to the slave processors which solve the Navier-Stokes equations in parallel. While both parallelisation approaches have been shown to produce reasonable performances, there exist some drawbacks and challenges. The strategy implemented by Uhlmann [164] requires continuous exchange of control on 
the Lagrangian mesh by the processors which may lead to a complex programming environment. The approach of Wang et al. [165] eliminates this issue leading to a simple structure of the code, increase in the memory usage on the master processor and data transfer between the master and slaves are some hurdles. In this work, we propose a different easy to implement parallelisation approach for the IBM where the information of all triangle nodes is present with all processors. But the computation required for each Lagrangian node/structure is performed only by specific processors depending on the type of computation that needs to be performed. In other words the allocation of processor for the IBM depends on the task that needs to be performed which results in a task-based parallelism for the FSI-IBM computation.

We will first describe in brief the parallelisation strategy employed for the flow solver and later explain the data structures and parallelisation implemented for the FSI-IBM. For the flow solver we employ domain decomposition and split the Cartesian box into slabs i.e. 'one-dimensional slab' parallelisation. It is also possible to use a Cartesian box decomposed with 'two-dimensional pencil' parallelisation as shown in [31]. In such a fluid solver, the viscous terms are computed explicitly in the periodic directions to take advantage of the reduced ALL-to-ALL communications. This may prove fatal dispersed deforming bodies in the flow since we also need to resolve the boundary layers over the immersed bodies which have dominant velocity gradients. With pure MPI, slab based codes already give good scalability up to $10^{3}$ cores and this can be further enhanced to $10^{4}$ cores by having a hybrid MPI+OpenMP type parallelisation which is already sufficient to tackle a large scale of problems.

In addition to the slabs, each processor needs to store information from the neighbouring processors which would be required for computing the derivatives and is stored in what is called as a 'halo/ghost' layer. Since the flow solver employs a second-order finite difference spatial discretisation at most one halo layer is required on each side of a slab for single phase flows. However, as we explain later when this solver is coupled with a FSI-IBM solver for finite-size bodies which makes use of MLS interpolations, multiple halo layers become necessary. It is important to keep in mind that an unrestricted increase in the number of stored halo layers would automatically result in an increase in the communication time which may deteriorate the overall performance of the code. For the MLS interpolations which need a support domain of $27(3 \times 3 \times 3)$ Eulerian points at most 3 halo layers are necessary.

Consider two arbitrarily shaped deformable bodies immersed in a flow as shown in figure 5.5, where the squares represent the Lagrangian markers/centroids of the triangular elements. The allocation of Eulerian slabs to each processor is straight forward as the Eulerian mesh stays fixed in time and this is done at the start of the 


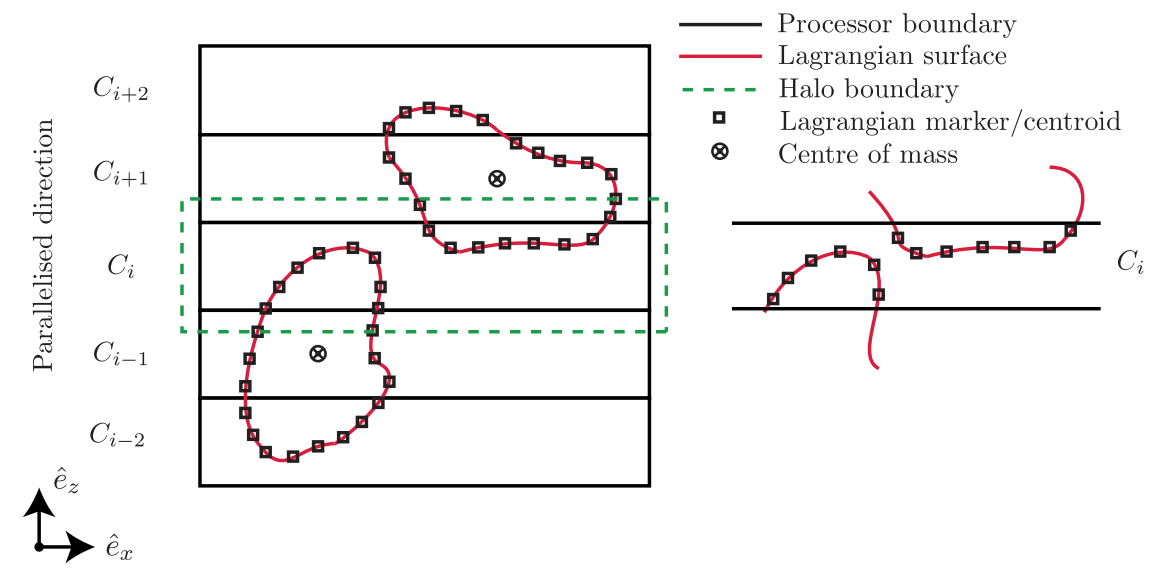

Figure 5.5: Schematic of two bodies immersed in a flow. Flow solver is 1D slab parallelised. $\hat{e}_{z}$ is the wall-normal direction.

simulation. For the flow solver each processor with identity myid is allocated the task of solving equation 1.1 on a slab of [1:N_1,1:N_2,N_3_start:N_3_end]. Given below are the steps undertaken to complete one full time step of the simulation. As given below there are four major steps and multiple sub-steps involved in completing one full iteration. The steps shown here are applicable for a loosely coupled approach which has been used for the simulations in this paper; details on the strongly coupled approach are elaborated in the paper by de Tullio and Pascazio [139].

1. Compute the indices of all markers/centroids on the Lagrangian mesh relative to the Eulerian mesh.

2. Compute the properties of the Lagrangian mesh, i.e. surface areas and normals of each face of the Lagrangian mesh.

3. Compute flow configuration along with IBM forcing i.e. all three sub-steps of RK3 integration.

(a) Compute intermediate fluid velocity under the RK3 framework.

(b) Interpolate velocity on the centroids of the Lagrangian mesh using MLS interpolation.

(c) Communicate the forces in the halo cells to neighbouring processors.

(d) Correct intermediate velocity using the MLS-interpolated force. 
(e) Solve pressure correction equation and compute the pressure and solenoidal velocity field.

4. Compute external and internal loads on the immersed body.

(a) Compute the external loads which is the sum of pressure and viscous forces on each face using MLS interpolation.

(b) Sum up external loads on all faces across all processors.

(c) Compute internal loads which are derived from the potentials described in section 5.2 .

(d) Sum up internal loads across all processors.

(e) Update the nodes of the triangles using Newton's law of motion.

The Lagrangian meshes shown in figure 5.1 are unstructured and are exported in the form of a GTS (GNU Triangulated Surface) data format which contains information about the spatial positions of the vertices of the triangular elements, the various vertices which are connected by edges and also the edges which constitute a face. Using this information we construct additional auxiliary arrays which will be required while computing the total force acting on the triangle nodes based on the potentials described in the section 5.2. The total number of vertices, edges and faces on a single immersed body is stored in N_vert, N_edge, N_face, respectively while N_particle is the total number of immersed bodies to be simulated and N_edge_vert is the maximum number of edges that any single vertex can be connected to. A brief overview of the required auxiliary integer arrays is given below.

1. vert_of_edge [2, N_edge, N_particle] : Contains pairs of vertices sharing a single edge.

2. face_of_edge [2, N_edge, N_particle] : Contains pairs of faces sharing a single edge.

3. vert_of_face[3, N_face, $N_{-}$particle] : Contains the three vertices that constitute a single face.

4. edge_of_face[3, N_face, N_particle] : Contains the three edges that constitute a single face.

5. vert_of_vert[N_edge_vert, N_vert, N_particle] : Contains all the vertices that a single vertex is connected to. 
6. edge_of_vert [N_edge_vert, N_vert, N_particle] : Contains all the edges that a single vertex is connected to.

7. v1234[4, N_edge, N_particle] : Contains all the four vertices that is contained in two faces sharing an edge.

8. pind [3, N_face, N_particle] : Stores the [N_x,N_y,N_z] indices of each centroid relative to the Eulerian mesh and tells us inside which Eulerian computational cell the centroid resides in. This array is updated every time step.

9. bboxind[6,N_particle] : Stores the indices of the bounding box of each immersed body.

In the first step, we compute the indices of all the centroids on every triangular element and store it in a global array pind. In addition to the axial index of every triangular element we also compute the mean axial index of every immersed body i.e for an immersed body $i$ the mean axial index is bboxind $[1: 3, i]=0.5 *(\max (\operatorname{pind}[1: 3,:, i]+\min (\operatorname{pind}[1: 3,:, i]))$. In step 2 , we compute the geometrical properties of the triangulated mesh (i.e. surface areas and normals of each triangular element). Both steps (1 and 2) are done by all processors (i.e. MPI_COMM_WORLD) on all immersed bodies and at the end of this operation every processor has information on all three indices [pind (1:3,N_face,N_particle)] of every centroid immersed in the flow, surface areas and normals of every triangular element.

For steps 3(a), 3(d) and 3(e) each processor performs all the operations required on its respective slabs. Step 3(b), which consists of interpolation using MLS and computing the IBM force has to be performed on the Lagrangian markers (centroids here) and this is done only on the centroids lying within the processors slab (c.f. right panel of figure 5.5). This allocation is regardless of which immersed body it belongs to. This is achieved by first performing a check on the axial index of every centroid (stored in pind [3,:,:] and computed in step 1); for example, if the processor $C_{i}$ is responsible for the slab $\left[1: N_{-} 1,1: N_{-} 2, N_{-}\right.$3_start:N_3_end $]$the following procedure is undertaken.

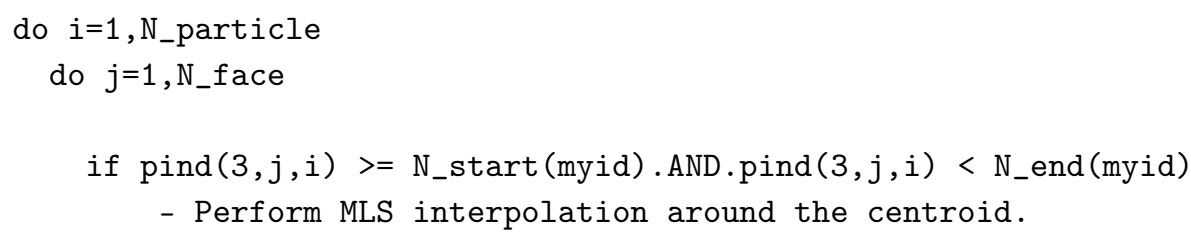


- Compute IBM forcing.

end if

end do

end do

As explained in section 5.2, MLS interpolations require a support domain built from 3 Eulerian grid nodes in each direction. Thus the forcing computed from a centroid lying right next to a processor boundary would be stored in a halo layer and this is communicated to the neighbouring processors in step 3(c). Every processor adds the IBM forcing received from the halo cells of the neighbouring processors to the already existing forcing thus accounting for the forcing from the centroids lying on processor boundaries.

Step 4(a) involves computing the external forces on the immersed body (i.e. pressure and viscous forces) which are performed following the procedure described in section 5.2. The allocation of processor for computing the external processors is done in a similar manner to step 3(b). Here it is important to note that for centroids lying on the processor boundaries the probes may lie in the neighbouring processor. For example, a centroid belonging to processor $C_{i}$ may have an axial index of N_3_start and the axial index of the corresponding probe would be N_3_start-1. Building a support domain around N_3_start-1 would require information from [N_3_start-2, N_3_start-1, N_3_start] i.e. at least two halo layers need to be stored by each processor.

do $i=1, N_{-}$particle

do $j=1$, N_face

if $\operatorname{pind}(3, j, i)>=N_{-} \operatorname{start}(m y i d)$. AND. pind $(3, j, i)<N_{-}$end (myid)

- Compute probe and build support domain around the probe.

- Perform MLS interpolation around the probe.

- Compute pressure and viscous forces on faces.

- Distribute the forces from faces to nodes.

end if

end do

end do

In step 4(b), we reduce the external forces $\left(\boldsymbol{F}_{\text {ext }}\right)$ over all the triangle nodes immersed in the flow. MPI_ALLREDUCE is used to perform this operation which results 
in all the processors having information on the external forces acting on all triangular nodes. Here it is important to remember that the efficiency of the MPI_ALLREDUCE operation may depend on the system architecture and checks are necessary before proceeding to large scale runs with this algorithm. The total force acting on each triangular node is computed as the summation of the external forces (pressure + viscous) and the internal forces arising from the elastic potentials i.e. $\boldsymbol{F}=\boldsymbol{F}_{\text {ext }}+\boldsymbol{F}_{\text {int }}$; for the first time step the immersed body is in its reference state and all internal forces are equal to zero and in every succeeding time step $F_{\text {int }}$ is the internal elastic forces computed in the previous time step. With this step every processor updates the position of the triangle nodes based on the total force.

Step 4(c) involves computing the internal elastic forces derived from the potentials on each immersed body. Since this requires the full body to be treated as a whole, we compute the location of the mean axial index of every individual immersed body from the information in the array pind. The processor responsible for this axial index takes care of computing all the internal elastic forces (i.e. in-plane deformation, out-of-plane deformation, volume constraint and area constraint) and computing the net internal force acting on each node belonging to its allocated immersed body. While computing the internal forces on each immersed body does not require any information from the Eulerian mesh, such an allocation ensures the computing load is distributed evenly across all processors. Also it is important to note that MLS interpolations which are the computationally expensive steps in this FSI-IBM code are still performed only by processors containing the Lagrangian markers. The pseudo code for this operation is given below.

do $i=1, N_{-}$particle

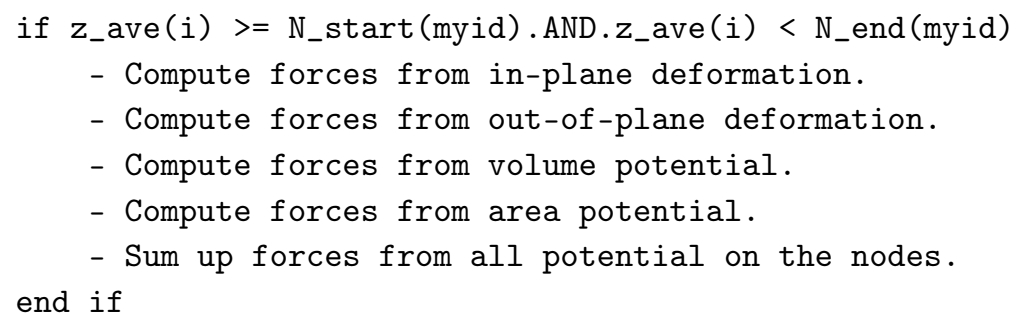

end do

In step $4(\mathrm{~d})$, we reduce the internal forces $\left(\boldsymbol{F}^{\text {int }}\right)$ over all triangle nodes with an MPI_ALLREDUCE operation similar to the operation in $4(\mathrm{~b})$. With this we complete all 
(a)

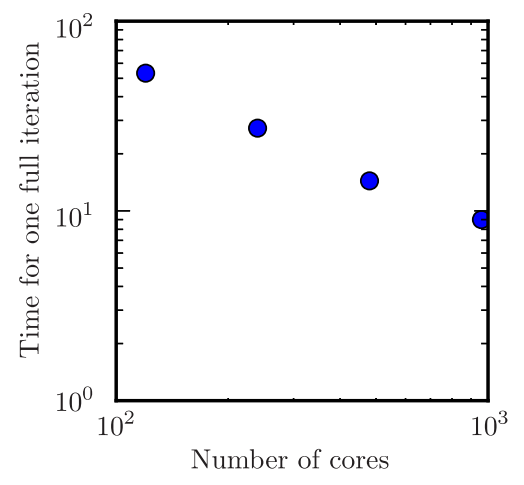

(b)

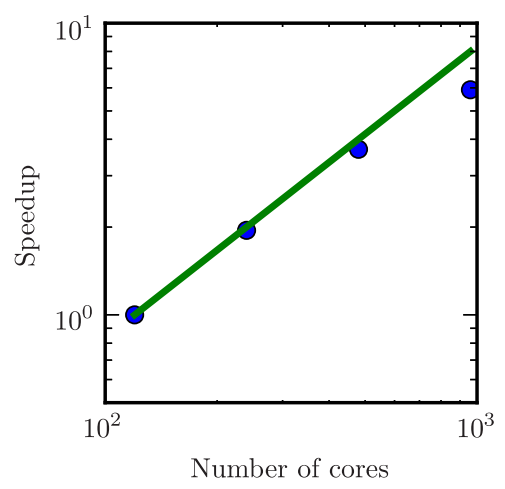

Figure 5.6: (a) Scaling plot showing the time step for one full iteration of the solver versus the number of cores used. (b) Corresponding speed up versus the number of cores.

the steps required for one full iteration of the flow solver and the IBM coupled with the deformation.

\subsubsection{Scaling}

In figure 6.3, we show the computational performance of the parallelisation strategy discussed in the Appendix. These simulations were performed on the thin nodes of the Dutch supercomputing facility 'Cartesius' where each node is composed of $2 \mathrm{x} 12$ core $2.6 \mathrm{GHz}$ Intel Xeon E5-2690 v3 CPU's. As can be seen from the plots in figure 6.3 strong scaling is achieved up to 1000 cores. The Eulerian resolution was set to $720 \times 720 \times 3840$ with a total of 25000 spherical particles each discretised using 320 faces, i.e. a total of 8 Million Lagrangian markers were simulated simultaneously. Due to global storage of the geometrical features and meta data of the Lagrangian markers, the memory consumption by the IBM-IP part of the flow solver for such simulation is approximately $600 \mathrm{MB}$.

For a given single phase flow solver, the costliest steps in the IBM part of the solver are the ones involving MLS interpolation since each interpolation requires the construction of multiple coefficient matrices and a subsequent inversion of a $4 \times 4$ matrix (in 3D). For each Lagrangian marker (centroid) immersed in the flow two MLS interpolations are required; one at the Lagrangian marker itself to compute the IBM forcing and another at the position of the probe projected from the centroid which is used for the computing the value pressure and velocity gradients. Additional matrix 

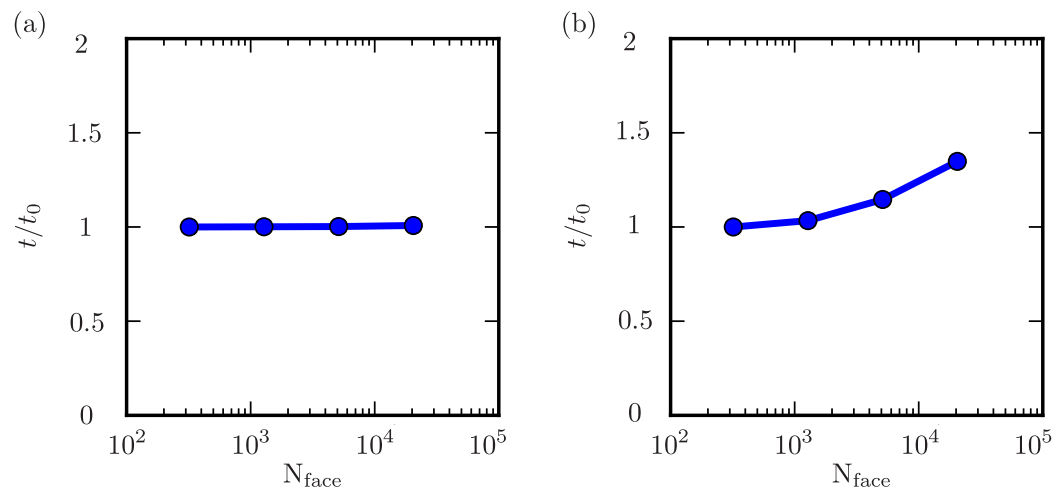

Figure 5.7: Time taken for one full iteration normalised using the time taken for the first data point versus the total number of triangular elements or faces immersed in the domain. In the left panel the immersed bodies are stationary and fixed in shape while in the right panel the bodies can both move and deform.

operations are required for the velocity gradients since instead of the shape function we need to compute the derivative of the shape function [143]. On a single processor, increasing the number of Lagrangian markers by two times can result in a three to five fold increase in the simulation time. It is thus crucial to see how the parallel code performs with increase in the total number of Lagrangian markers or triangular faces.

In figure 5.7 we plot the non-dimensional time taken for one full iteration with increasing total number of faces for two different types of simulations. The time is normalised using the time taken for the first data point i.e. $N_{\text {face }}=320$. For these simulations the Eulerian grid is kept fixed to $120 \times 120 \times 720$ and a total of 120 cores were used. In the left panel of figure 5.7, the immersed bodies are kept fixed in position and shape i.e. the computation of the structural solver is fully eliminated. Such simulations are useful to compute the hydrodynamic forces acting on stationary bodies with a mean flow imposed in the domain. As can be seen, with increase in the number of faces there is negligible increase in the computational time. In comparison to simulations involving moving and deforming objects, IBM simulations with stationary and fixed bodies require only one MLS interpolation and this is the reason for the negligible time increase. On the right panel we show the increase in time for simulations involving both moving and deforming bodies. These simulations require an additional MLS interpolation at the probe and also the computation of shape function derivatives. For such simulations, a 100 times increase in the number 
of faces results in approximately 1.3 times increase in the computational time. This shows that the increasing cost of MLS interpolations on the Lagrangian markers can be offset by parallelising the task over multiple processors.

\subsection{Summary}

In this chapter we have demonstrated the implementation of a finite-difference based flow solver capable of handling several deforming drops/bubbles where their deformation dynamics is computed through an interaction potential approach. An immersed boundary method based on moving least squares interpolation is used to enforce the interfacial boundary condition on the underlying flow while the deformation dynamics is computed through minimising the potential energy of a spring network spread over the surface of an immersed drop or a bubble. The unique feature of this approach in comparison to conventional techniques used to simulate immersed drops or bubbles is its computationally efficiency which allows us to scale up both in terms of the number of drops or bubbles in the flow and also the turbulence level in the surrounding fluid. In the first part of the paper we discuss briefly why the presented approach can replicate the deformation dynamics of immersed drops/bubbles and the estimation of ad-hoc elastic constants required in the model. By comparing with already existing analytical solutions and experimental measurements of standard configurations (deforming drop in a shear flow and cross flow), we have shown that such an approach is practical, reliable and self-consistent. To this effect, in the last part of the chapter, we present a parallelisation strategy which is easy to implement for any generic single phase fluid solver. With the pure MPI based parallelisation we have shown that decent scalability is achieved up to $10^{3}$ cores and can be further enhanced to $10^{4}$ by including OpenMP directives. 


\section{Chapter 6}

\section{A fast moving least squares approximation with adaptive Lagrangian mesh refinement for large scale immersed boundary simulations}

Based on: Vamsi Spandan, Detlef Lohse, Marco D. de Tullio, Roberto Verzicco, 'A fast moving least squares approximation with adaptive Lagrangian mesh refinement for large scale immersed boundary simulations' to be submitted to Journal of Computational Physics 


\subsection{Introduction}

Numerical simulations of flows around moving and deformable bodies with complex geometries have been an immense challenge to the fluid dynamics community for a long time. Computational studies using body-fitted methods where the mesh needs to be adjusted according to the instantaneous shape and position of the immersed body become not only mathematically complex but also terminally expensive [166]. This makes non body-fitted methods such as the immersed boundary method (IBM) very attractive to deal with complex flow systems where there is coupling between a carrier fluid and a deformable interface, membrane or a structure [127]. IBM uses a single time-invariant mesh for the solution of the Navier-Stokes (NS) equations and a separate set of nodes distributed on the surface of any immersed body. The influence of an immersed rigid or deformable body on the surrounding fluid is achieved through a spatially and time varying forcing function in the NS equations. This method was first used by Peskin [125] to study flow around heart valves and has gained immense popularity over the last two decades due to its ability to handle a large class of problems; for example suspension of rigid spheres in turbulent flows [128-131], deformable liquid-liquid interfaces and elastic membranes [139, 167], flow around bluff bodies [133] etc. One particular field where IBM has excelled is in the simulation of dispersed rigid particles in highly turbulent flows [128-131]. Recent works have shown that while the extension from rigid particles to deformable particles is highly non-trivial it can be achieved with the help of optimised deformation and parallelisation algorithms [132, 139, 167].

Fully resolved simulations of large scale multi-phase flows are useful not only to understand the fundamental features of the flow but also to benchmark and improve lower-order models which are used extensively in industry. Simulations of such systems which were not possible a decade ago are now being made possible with the combination of a variety of ingredients such as an efficient NS solver for the fluid-phase, an IBM with the ability to handle deformable bodies and also parallelisation of the complete flow solver. However, there is still a need to optimise several sub-components of the flow solver such as interpolation techniques, collision detection algorithms, mesh refinement, load balancing for parallel simulations etc. which are some of the major bottlenecks in computing long-time statistics for large scale simulations involving IBM (the term large scale can refer to numerical simulations involving $O\left(10^{4}\right)$ fully resolved deformable bodies with $O\left(10^{3}\right)$ Lagrangian markers each in a turbulent flow). In this chapter, we tackle some of these bottlenecks and introduce simple and effective optimisation algorithms within the immersed boundary framework which are 
geared towards scaling up simulations of highly turbulent flows with thousands of fully resolved deformable particles.

As mentioned earlier, IBM requires two different meshes; an Eulerian mesh for the solution of NS equations and a Lagrangian mesh on the surface of the immersed body which is primarily used to enforce the interfacial boundary condition. When the immersed bodies can deform, the Lagrangian mesh can also serve as the computational nodes for the discretisation of the deformation governing equations; such as the Cauchy-Navier equations in elastic deformation problems. While there are multiple variants of IBM which vary in how the influence of the immersed body is imposed on to the carrier fluid, the most popular approach in the case of moving bodies is the one introduced by [135]. In this approach, the forcing function in the NS equations is first computed on the Lagrangian nodes distributed on the surface of the immersed body and later spread onto the Eulerian mesh. Since the Lagrangian nodes need not coincide with the Eulerian mesh at every time instant interpolation and extrapolation algorithms are implemented into the flow solver. This brings into play a couple of issues. Firstly, it is important to ensure that the transfer functions used to exchange information between the Eulerian and Lagrangian meshes ensures conservation of momentum between the immersed body and the surrounding fluid in addition to maintaining a specific order of accuracy compatible with the flow solver. Secondly, the calculation of the transfer function should not become compute intensive as this hampers the ability of the flow solver to handle large scale systems. Another major issue in numerical simulations involving IBM is the coupling or in other words dependence of the resolution of the Lagrangian mesh on the Eulerian mesh. Having a Lagrangian resolution coarser than that of the Eulerian resolution results in a discontinuous representation of the immersed boundary which leads to artificial effects in the flow. Typically, the acceptable choice is that the length scale of an individual Lagrangian element is set such that it is equal to 0.7-0.9 times that of the local Eulerian grid size.

In recent works, the moving least squares (MLS) approximation has been used to build the transfer functions between the Eulerian and Lagrangian meshes and in particular has been favoured for the simulation of deformable interfaces and membranes [137, 139, 167]. MLS allows for interpolating both the value and the spatial derivatives of a flow variable at any given point based on a set of field nodes defined within a local support domain in addition to ensuring the transfer function is smooth in presence of moving and deforming boundaries. MLS was first introduced by mathematicians to solve the problem of data fitting and surface reconstruction and given its highly generic nature it has been used in several fields thereafter 
[137, 139, 144-153, 167]. While MLS provides several advantages in handling moving and deformable bodies in inhomogeneous unsteady flows, there are certain inherent issues in the algorithm which make it difficult to use freely. In comparison to conventional interpolation techniques MLS is an operation intensive algorithm as it requires the construction of multiple weight matrices and a matrix inversion for every Lagrangian marker where the approximation is required. This severely hampers the wall-clock time of the simulation when there are $O\left(10^{6}-10^{7}\right)$ Lagrangian elements immersed in the flow. In the first part of this chapter, we introduce a simple algorithm (referred to as fast-MLS) where we compute the MLS shape functions at the start of the simulation at pre-defined nodes and then use a relatively inexpensive interpolation technique (Shepard's interpolation here) to estimate the shape function at any arbitrary Lagrangian marker. With this we aim to achieve the smoothness and accuracy of MLS shape functions while eliminating the operation costs of constructing and inverting weight matrices in the exact-MLS approach. We compare the results and the total computational time from both approaches for the well-studied problem of uniform flow over a stationary sphere.

As mentioned earlier, due to the coupled nature of the Eulerian and Lagrangian meshes in IBM simulations, any increase in the Eulerian mesh forces an increase in the Lagrangian mesh. This can severely hamper the performance of the code when the immersed bodies are deformable and the Lagrangian mesh is used to discretise the deformation governing equations. In order to tackle this problem, we propose segregating the Lagrangian mesh into two parts (i) base mesh which can be used to deform the immersed body (ii) refined mesh for computing the forcing function and avoiding discontinuities in the interfacial boundary condition. The base mesh needs to be initialised only once during the start of the simulation while the refined mesh is built on top of the base mesh according to the local Eulerian resolution. This approach not only makes the flow solver more flexible since the deformation governing equations can now be discretised independently of the Eulerian mesh, it also reduces the memory load on the code along with lower computational costs. To test the validity of the proposed approach, we compare results of a deforming drop in a cross-flow by both fully resolving the Lagrangian mesh according to the Eulerian resolution and also using the refinement technique which requires relatively lesser operations and memory.

When simulating thousands of deformable bodies dispersed in a moving fluid, detecting and modelling collision between them becomes a challenging computational task. The algorithms and models required to satisfactorily detect and model the collisions have received considerable attention in the past given its wide range of 
applications such as video games, physics based animations, event driven simulations, multiphase flows etc. There exist a number of problem specific techniques in literature which can be used to detect collision between any two points residing on different objects; see [168] for a detailed review. The brute force approach is to compute the distance between a marker on one body against every other marker on other bodies to determine a collision event. Using such a simple and straight-forward technique comes at a cost, as the number of operations required to detect a collision becomes terminally expensive with increasing number of markers. Significant progress has been made in the field of molecular dynamics where the typical approach is to maintain a list of neighbouring particles within a predetermined support radius with whom collision is likely and then update this list periodically throughout the simulation [169]. While this a more efficient and computationally inexpensive procedure where the total operation count is $O(N \log (N))$ as compared to $O\left(N^{2}\right)$ operations in the brute force method, it still adds additional overhead to the total simulation time per iteration. In the last part of the paper, we discuss a simple and easy-to-implement coarse grained collision detection algorithm which can be used to detect collision events between markers residing on different deformable bodies immersed in a flow resolved by a Eulerian grid with minimal computational overhead. We are primarily focussed towards collision detection between deformable bodies within the immersed boundary framework i.e. the fluid surrounding the immersed body is discretised using an Eulerian grid while the dispersed bodies are discretised using a separate Lagrangian mesh.

This chapter is organised as follows. In the next section we describe the fast moving least squares (f-MLS) approach and compare the results from this approach with the conventional MLS-IBM simulations. In section 3, we describe the adaptive Lagrangian mesh refinement technique and compare the results from this technique with previous works. In section 4, we discuss the details and results from the coarse-grained collision detection algorithm and then provide a summary in section 5 .

\subsection{Fast moving least squares for the immersed boundary method}

To be consistent we once again describe here briefly the steps involved in a MLS approximation (refer to $\S 5$ for further details). In MLS, a field variable $q$ given on a pre-defined set of nodes is approximated at any desired point lying within these nodes and positioned at $\mathbf{x}_{l}$ as follows: 


$$
Q\left(\mathbf{x}_{l}\right)=\mathbf{p}^{T}\left(\mathbf{x}_{l}\right) \mathbf{a}\left(\mathbf{x}_{l}\right)
$$

$Q$ is the approximated quantity while $\mathbf{p}^{T}\left(\mathbf{x}_{l}\right)$ is a basis function vector with dimension $m$. $\quad \mathbf{a}\left(\mathbf{x}_{l}\right)$ is the vector of coefficients obtained by minimising the weighted L2 norm as described later and $\mathbf{x}_{l}$ is the position vector of the Lagrangian marker $l$. $\mathbf{p}^{T}\left(\mathbf{x}_{l}\right)=\left[p_{1}\left(\mathbf{x}_{l}\right), \ldots, p_{m}\left(\mathbf{x}_{l}\right)\right]$ is built using the Pascal's triangle and pyramid in two and three-dimensions, respectively. In this work, we consider a linear basis function with $\mathbf{p}^{T}(\mathbf{x})=[1, x, y, z]$, i.e. $m=4$ which has already been shown to be a cost-efficient and reliable choice for flow simulations incorporating the immersed boundary method $[137,139,167]$. It is to be remembered that the fast-MLS approach described later is independent of the choice of $m$.

The matrix $\mathbf{a}\left(\mathbf{x}_{l}\right)$ is computed by minimising a weighted residual $J$ which is defined as follows:

$$
J=\sum_{k=1}^{N_{e}} W\left(\mathbf{x}_{l}-\mathbf{x}_{k}\right)\left[\mathbf{p}^{T}\left(\mathbf{x}_{k}\right) \mathbf{a}\left(\mathbf{x}_{l}\right)-q_{k}\right]^{2}
$$

$N_{e}$ is the number of nodes in the Eulerian grid which are in support of the Lagrangian marker $l$ and the selection of these nodes is described later. $W\left(\mathbf{x}_{l}-\mathbf{x}_{k}\right)$ is a weight function for point $\mathbf{x}_{k}$ and can be computed in different ways; for example using exponential functions, cubic splines, quadratic splines etc. [143]. Minimising $J$ with respect to $\mathbf{a}\left(\mathbf{x}_{l}\right)$ leads to the relation $\mathbf{A}(\mathbf{x}) \mathbf{a}(\mathbf{x})=\mathbf{B}(\mathbf{x}) \mathbf{q}$, where $\mathbf{q}$ is a vector containing the values of the field variable $q$ on the pre-defined set of nodes; $\mathbf{A}(x)$, $\mathbf{B}(x)$ and $\mathbf{q}$ are defined as follows:

$$
\begin{gathered}
\mathbf{A}\left(\mathbf{x}_{l}\right)=\sum_{k=1}^{N_{e}} W\left(\mathbf{x}_{l}-\mathbf{x}_{k}\right) \mathbf{p}\left(\mathbf{x}_{k}\right) \mathbf{p}^{T}\left(\mathbf{x}_{k}\right) \\
\mathbf{B}\left(\mathbf{x}_{l}\right)=\left[W\left(\mathbf{x}_{l}-\mathbf{x}_{1}\right) \mathbf{p}^{T}\left(\mathbf{x}_{1}\right) \ldots W\left(\mathbf{x}_{l}-\mathbf{x}_{N_{e}}\right) \mathbf{p}^{T}\left(\mathbf{x}_{N_{e}}\right)\right] \\
\mathbf{q}=\left[q_{1} \ldots q_{N_{e}}\right]^{T}
\end{gathered}
$$

Minimising equation 6.2 with respect of $\mathbf{a}(\mathbf{x})$ gives the coefficient vector $\mathbf{a}(\mathbf{x})=$ $\mathbf{A}^{-1}(\mathbf{x}) \mathbf{B}(\mathbf{x}) \mathbf{q}$ and the approximated value of the field variable $q$ at $\mathbf{x}_{l}$ can be computed as follows:

$$
Q=\mathbf{p}^{T}(\mathbf{x}) \mathbf{A}^{-1}(\mathbf{x}) B(\mathbf{x}) \mathbf{q}
$$

The approximation in equation 6.6 can also be written as $Q=\boldsymbol{\Phi}^{T}(\mathbf{x}) \mathbf{q}$, where $\boldsymbol{\Phi}^{T}(\mathbf{x})$ is a vector of shape function coefficients of size $1 \times N_{e}$ and is computed as $\boldsymbol{\Phi}^{T}(\mathbf{x})=$ 
$\mathbf{p}^{T}(\mathbf{x}) \mathbf{A}^{-1}(\mathbf{x}) \mathbf{B}(\mathbf{x})$. This shape function is computed for every Lagrangian marker in the flow and is used to compute the approximate value of the required Eulerian quantity (typically fluid velocity) at the position of the marker. Approximation of the fluid velocity gives information on the magnitude of the forcing that needs to be transferred to the Eulerian grid which ensures that the interfacial boundary condition is satisfied. If the vector $\mathbf{q}=\left[u_{1} \ldots u_{N_{e}}\right]^{T}$, where $u_{k}$ is the fluid velocity defined at the Eulerian nodes and its corresponding approximated quantity $Q=U$, the total IBM force corresponding to the Lagrangian marker $l$ is written as $\mathbf{F}_{l}=\left(\mathbf{V}^{b}-\mathbf{U}\right) / \Delta t$. In case of no-slip boundary condition at the interface of the immersed boundary, $\mathbf{V}^{b}$ would be equal to the velocity of the Lagrangian marker. Once the forcing has been determined, the next step is to distribute it onto the Eulerian grid in such a way that the total force during the transfer is conserved which leads to the following relation.

$$
\sum_{k=1}^{N_{e}} \mathbf{f}_{k} \Delta V_{k}=\sum_{l=1}^{N_{l}} \mathbf{F}_{l} \Delta V_{l}
$$

$\Delta V_{k}$ is the volume of the Eulerian cell $k$ and $\Delta V_{l}=A_{l} h_{l}$ is the forcing volume associated with the Lagrangian marker $l ; A_{l}$ is the surface area of the Lagrangian marker $l$ and $h_{l}=\sum_{k=1}^{N_{e}} \phi_{k}^{l}\left(\Delta x_{k}+\Delta y_{k}+\Delta z_{k}\right) / 3$. Once the shape function is computed, the forcing to be included in the Eulerian cell $k$ is computed as $\mathbf{f}_{k}=\sum_{l=1}^{N_{l}} c_{l} \phi_{k}^{l} \mathbf{F}_{l}$. The scaling factor $c_{l}$ is calculated such that equation (6.7) is satisfied and is given as follows:

$$
c_{l}=\frac{\Delta V_{l}}{\sum_{k=1}^{N_{e}} \phi_{k}^{l} \Delta V_{k}}
$$

The construction of the auxiliary matrices $\mathbf{A}(x)$ and $\mathbf{B}(x)$ followed by an inversion of $\mathbf{A}(x)$ are the computationally most intensive parts of the MLS approximation. For stationary bodies, a single MLS interpolation per Lagrangian marker per time step of the Eulerian fluid velocity is sufficient. However, when the immersed bodies can move and deform we also need to interpolate the pressure and fluid stress tensor at the Lagrangian marker which leads to additional MLS operations. Since these steps have to be performed for every individual Lagrangian marker immersed in the flow it results in a massive increase in the computational time with increasing Lagrangian resolution. In the fast-MLS approach we eliminate the process of computing the shape function $\boldsymbol{\Phi}(x)$ during the simulation and construct them only at the start of every simulation for a pre-defined set of nodes. The shape function for any arbitrary Lagrangian 
(a)

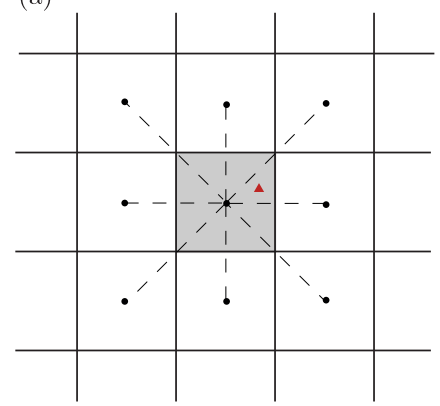

(b)

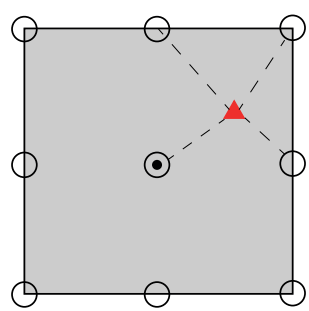

(c)

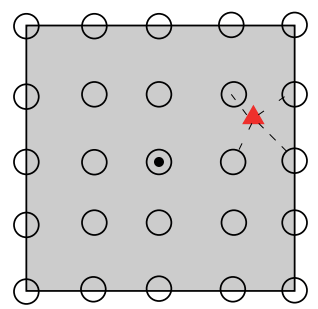

Figure 6.1: (a) Schematic of a two-dimensional support domain of the Lagrangian marker (shown by red triangle); dotted lines show all the Eulerian cell centres in support of the Lagrangian marker. (b) Home Eulerian cell of the Lagrangian marker discretised by $N_{i}=9$ and (c) $N_{i}=25$ sub-Eulerian nodes, respectively.

marker is then computed through a relatively inexpensive interpolation procedure such as tri-linear interpolation or the Shepard's interpolation. The procedure for selecting the nodes used for computing the shape functions and interpolation of the shape functions is now detailed.

In figure 6.1(a) we show the schematic of a two-dimensional support domain used for an arbitrary Lagrangian marker. For the sake of brevity, in the following explanation, we consider a two-dimensional system; extension of the algorithm to three dimensions is straight forward. The support domain for an exact-MLS approximation in a two-dimensional system consists of $N_{e}=9(3 \times 3)$ Eulerian cells. The support domain is built by first identifying the home cell of the Lagrangian marker (indicated by the grey region in figure 6.1(a)) and then taking into consideration its neighbouring cells. In fast-MLS we take advantage of the fact that the shape functions of Lagrangian markers at the same relative distances to the Eulerian cell centres of their respective support domains will be similar.

To incorporate the fast-MLS approach, we first discretise a single Eulerian cell using an imaginary mesh (hereafter called the sub-Eulerian mesh) before the start of the simulation as shown by hollow circles in figure $6.1(\mathrm{~b})$; in this case, the resolution of the sub-Eulerian mesh is $N_{i}=9$ points. Next, we compute the exact shape functions at each of these points at the start of the simulation using the algorithms described previously, i.e. equations (6.3)-(6.6). This leads to nine different exact shape function vectors which contain nine coefficients each. During the simulation, the location of the Lagrangian marker is identified with respect to the sub-Eulerian mesh in its home 
cell and the shape function is estimated as follows:

$$
\begin{gathered}
\boldsymbol{\Phi}_{f}\left(\mathbf{x}_{l}\right)=\sum_{i=1}^{4} \alpha_{i} \boldsymbol{\Phi}_{e}^{i}\left(\mathbf{x}_{i}\right) \\
\alpha_{i}=\frac{1 / d_{i}^{2}}{\sum_{i=1}^{4} 1 / d_{i}^{2}}
\end{gathered}
$$

$\mathbf{\Phi}_{f}\left(\mathbf{x}_{l}\right)$ is the shape function estimated from the fast-MLS approach, $\mathbf{\Phi}_{e}\left(\mathbf{x}_{i}\right)$ is the exact shape function calculated at the nodes of the sub-Eulerian mesh at the start of the simulation, $\mathbf{x}_{i}$ is the position of the node $i$ on the sub-Eulerian mesh; $\alpha_{i}$ are the coefficients of interpolation computed through Shepherd's interpolation which is a relatively inexpensive interpolation technique and depends only on $d_{i}$ which is the distance between the Lagrangian marker and the nodes of the sub-Eulerian mesh in consideration; $N_{i}$ is the number of nodes taken into consideration from the sub-Eulerian mesh. The sub-Eulerian mesh can be further refined and discretised using 25 points as shown in figure 6.1(c) in which case the shape functions on 25 $(5 \times 5)$ different nodes with 9 coefficients each would need to be stored at the start of the simulation. The interpolation of the shape function according to equation 6.9 would then be computed based on a different set of nodes. The next level of refinement of the sub-Eulerian mesh would consist of $81(9 \times 9)$ nodes.

We now compare the shape function coefficients calculated using both fast-MLS and exact-MLS techniques. In figure 6.2(a) we show the normalised maximum difference in the shape function coefficients versus the number of grid points used in the sub-Eulerian mesh. We see that with increasing sub-Eulerian mesh resolution there is a rapid decrease in the difference between the shape functions computed from the exact-MLS and the fast-MLS approaches. For a sub-Eulerian mesh discretised using $81(9 \times 9)$ points the error in the shape function coefficients is already below $3 \%$. We now use the fast-MLS technique to compute the drag coefficient of a three-dimensional stationary sphere facing a uniform stream of flow. The error in the shape function coefficients in a three dimensional system follows the same trend as shown in figure 6.2(a), while the only difference is the increase in the number of $N_{i}$, the sub-Eulerian grid nodes. In figure 6.2(b), we compare the drag coefficient of a three dimensional fixed rigid sphere versus the Reynolds number which is defined as $R e=U D / \nu$ ( $U$ is the free stream velocity, $D$ is the diameter of the sphere and $\nu$ is the kinematic viscosity of the fluid). A good agreement can be seen between the drag coefficients obtained from the simulations involving the fast-MLS and exact-MLS 
(a)

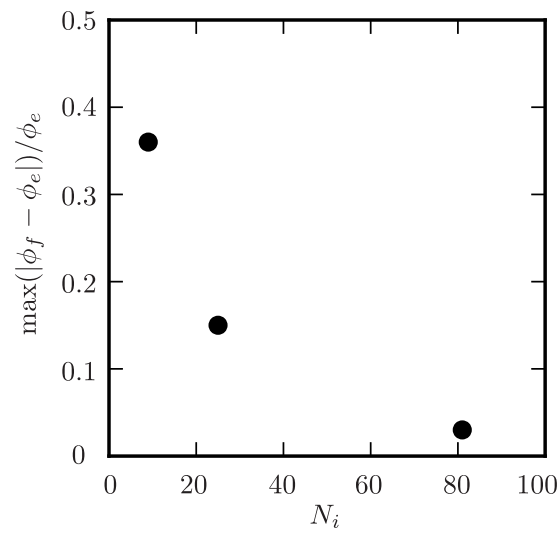

(b)

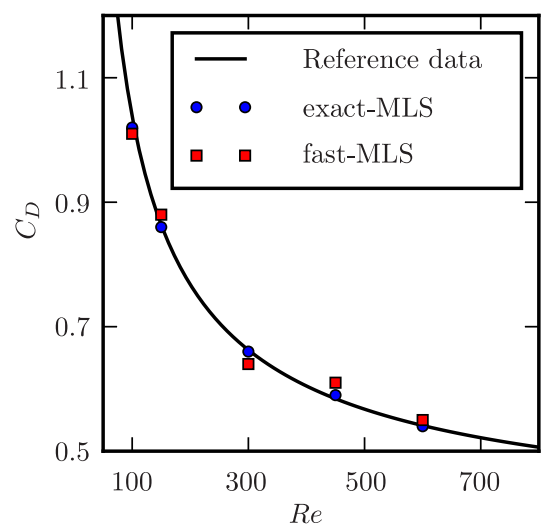

Figure 6.2: (a) Maximum difference in the shape function coefficients computed from the fast-MLS $\left(\phi_{f}\right)$ and the exact-MLS $\left(\phi_{e}\right)$ techniques versus the number of grid points used in the sub-Eulerian mesh. (b) Drag coefficient $C_{D}$ of a stationary rigid sphere facing a uniform flow stream versus the Reynolds number Re.

approach.

In figure 6.3(a), we show a comparison of the computational performance of the flow solver when using fast-MLS and exact-MLS techniques. The parallelisation algorithms and data structures used in this solver has already been described in $\S 5$ [167]. These simulations were performed on the thin nodes of the Dutch supercomputing facility 'Cartesius' where each node is composed of $2 \times 12$ core 2.6 GHz Intel Xeon E5-2690 v3 CPU's. The Eulerian resolution was set to 720x720x3840 with a total of 16000 spherical particles each discretised using 320 faces, i.e. a total of 5 Million Lagrangian markers were simulated simultaneously. It can be seen that the fast-MLS approach while ensuring good scalability also reduces the total wall clock time per time step. Here, one full iteration consists of integrating the fluid and immersed body governing equations for one time-step. In order to further analyse the computational boost provided by the fast-MLS approach we now fix the Eulerian resolution and increase the total number of Lagrangian markers $\left(N_{f}\right)$ in the flow. For these simulations the Eulerian grid is kept fixed to 120x120x720 and a total of 120 computing processors were used. In figure $6.3(\mathrm{~b})$ we plot the non-dimensional time taken for one full iteration with increasing number of faces where the time is normalised using the time taken for the first data point i.e. $N_{\text {face }}=320$. Here it is important to point out that for these simulations the immersed bodies can both move 

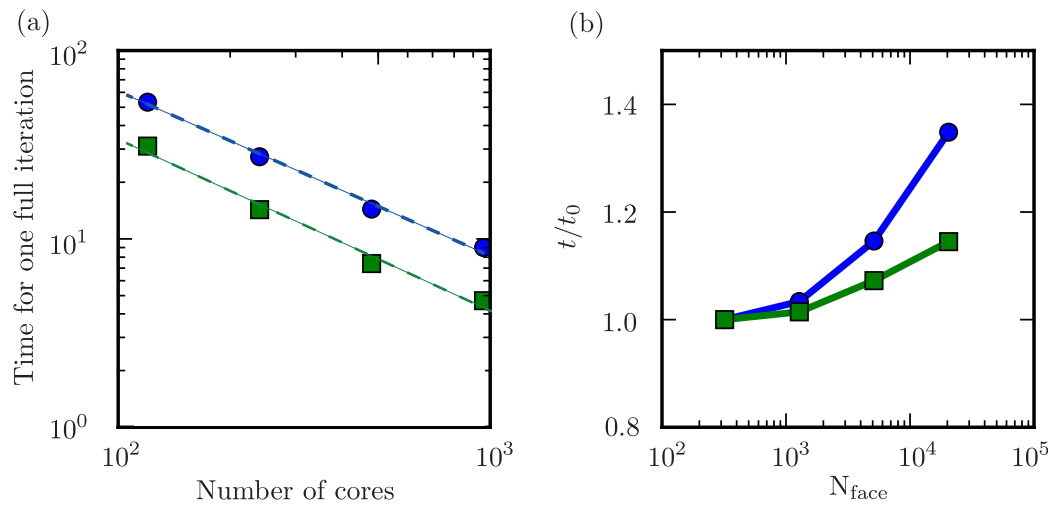

Figure 6.3: (a) Scaling plot showing the time step for one full iteration of the solver versus the number of cores used for both the exact-MLS and fast-MLS techniques. Circles correspond to exact-MLS while square markers correspond to fast-MLS. (b) Comparison of the total time taken for one full iteration versus the total number of Lagrangian markers immersed in the flow. $t$ is normalised using the time taken for the first data point at $N_{f}=162$.

and deform according to the deformation algorithm discussed by [167]. It implies that without the fast-MLS approach a single iteration would consist of multiple exact-MLS operations. It is seen from figure 6.3(b) that with approximately 20000 Lagrangian markers in the flow the total time taken per time step is reduced by at least $40 \%$ with the fast-MLS approach.

\subsection{Decoupling the Lagrangian meshes}

We now move on to describing the technique of adaptive Lagrangian mesh refinement and how it can be used in simulations involving IBM. The purpose of the refinement is to tackle two challenges in IBM simulations. Firstly, in IBM simulations, the resolution of the Lagrangian mesh is inherently coupled to the Eulerian mesh due to the continuous transfer of information between both meshes during the simulation. If the Lagrangian mesh is too coarse, the Eulerian flow field is not forced uniformly which leads to discontinuities (often called 'holes') in the interfacial boundary condition. An extremely fine Lagrangian resolution relative to the local Eulerian resolution helps solve this problem but leads to a massive increase in the total computational time. Typically, the optimal choice is such that the size of the Lagrangian elements is of the same size or slightly smaller than the Eulerian 
(a)

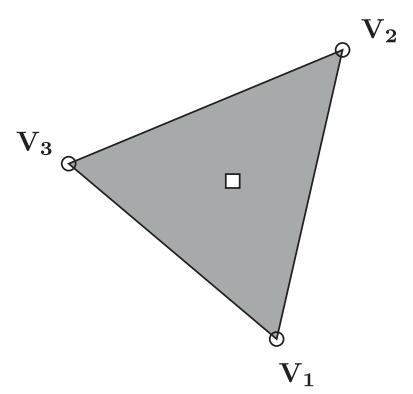

(c)

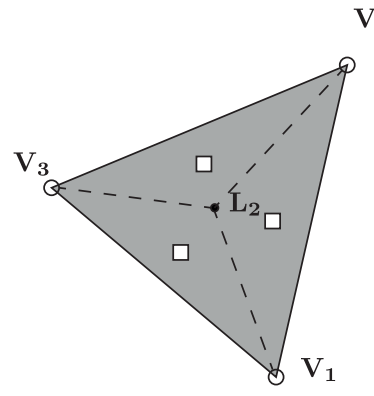

(b)
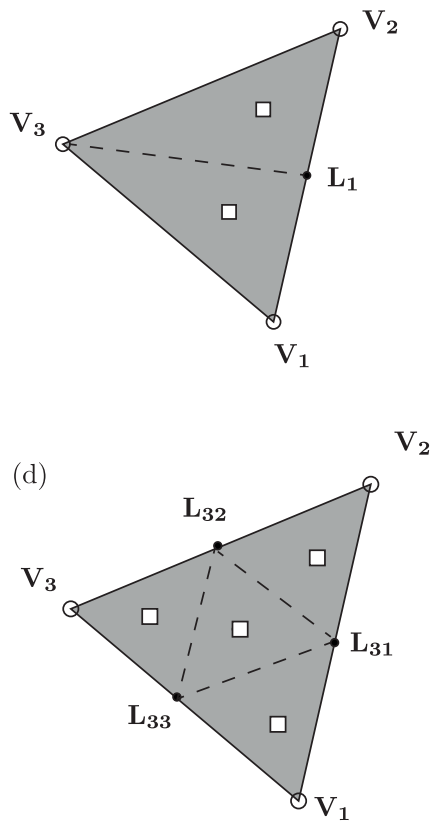

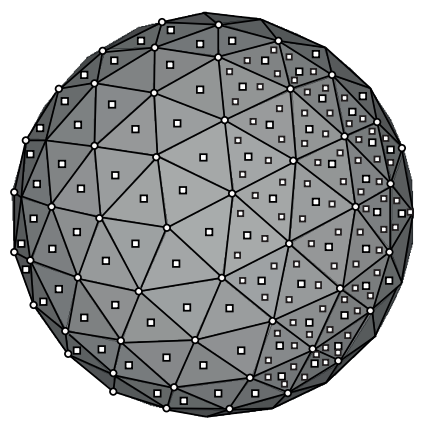

Figure 6.4: Triangulations for the adaptive Lagrangian mesh refinement. (a) initial base triangular element. (b), (c), (d) show the first, second and third level of refinements, respectively. Bottom panel shows a sphere where the right half of the sphere is refined using the third level while the left half still has its initial base mesh. 
grid resolution. Secondly, if the immersed body can also move and deform, the same Lagrangian mesh that is used for enforcing the interfacial boundary condition (IBM mesh) is used for the structural solver. This implies that a refinement in the IBM mesh automatically means a refinement in the structural solver mesh which may lead to unnecessary computational nodes for discretising the deformation governing equations. Here, we focus on describing the refinement procedure and the results from the simulations of a deforming drop with and without refinement.

The basic idea of the Lagrangian mesh refinement is shown in figures 6.4(a)-(d). Consider a triangular element as shown in figure 6.4(a) which is composed of the three vertices $\left(\mathbf{V}_{1}, \mathbf{V}_{2}, \mathbf{V}_{3}\right)$ and a centroid (represented by a square symbol). Given the vertices of any triangular element, the position of the centroid can be directly computed as $\mathbf{C}=\left(\mathbf{V}_{1}+\mathbf{V}_{2}+\mathbf{V}_{3}\right) / 3$ We can further refine the triangular element in multiple levels as shown in figures 6.4(b)-(d). In the first level of refinement (figure $6.4(\mathrm{~b}))$, the triangle is split into two such that $\mathbf{L}_{1}=0.5\left(\mathbf{V}_{1}+\mathbf{V}_{2}\right)$. In the next level of refinement (figure 6.4(c)) the triangular element is split in such a way that $\mathbf{L}_{2}$ is equivalent to the centroid of the triangular element i.e. $\mathbf{L}_{2}=\left(\mathbf{V}_{1}+\mathbf{V}_{2}+\mathbf{V}_{3}\right) / 3$. Similarly the triangle can further refined as shown in the figure $6.4(\mathrm{~d}) ; \mathbf{L}_{31}=0.5\left(\mathbf{V}_{1}+\right.$ $\left.\mathbf{V}_{2}\right), \mathbf{L}_{32}=0.5\left(\mathbf{V}_{2}+\mathbf{V}_{3}\right), \mathbf{L}_{33}=0.5\left(\mathbf{V}_{3}+\mathbf{V}_{1}\right)$. Here it is important to note that such a refinement procedure is computationally inexpensive and for a given base mesh as shown in figure 6.4(a) the positions of the vertices and centroids of the refined triangles can be computed very easily during the simulation as described above. A comment is necessary here regarding the refinement procedure used in figure 6.4(b), (c). If there is a large difference in the lengths of the three sides of the initial triangular element, then the refinements used in figure 6.4(b), (c) may lead to triangles with very small interior angles. This is not desirable as this would lead to a non-uniform distribution of the Lagrangian markers and consequently a discontinuous forcing. In order to avoid this, we ensure during the simulation that the interior angle of any refined triangle is above a certain threshold.

We now test this approach for a deforming drop with its centroid fixed immersed in a cross-flow and compute the mean shape arising from the resulting flow conditions with refinement and without refinement. The control parameters for such a problem are the Reynolds number, $R e=U d_{\mathrm{eq}} / \nu_{f}$ and the Weber number, $W e=\rho_{f} U_{\mathrm{ref}}^{2} d_{\mathrm{eq}} / \sigma$. For such a flow, the aspect ratio of the deforming drop depends on the Weber number $W e=\rho_{f} U^{2} d_{\mathrm{eq}} / \sigma$, which is the ratio of inertia forces acting on the drop in comparison the surface tension forces. The response of the system can be measured through the quantification of the morphology of the drop. The combined action of the dynamic pressure acting on the faces of the drop and the shear stresses generated from the 
(a)

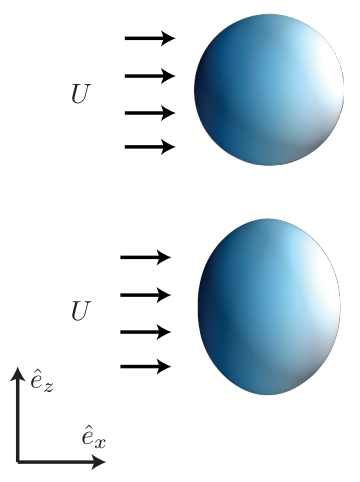

(b)

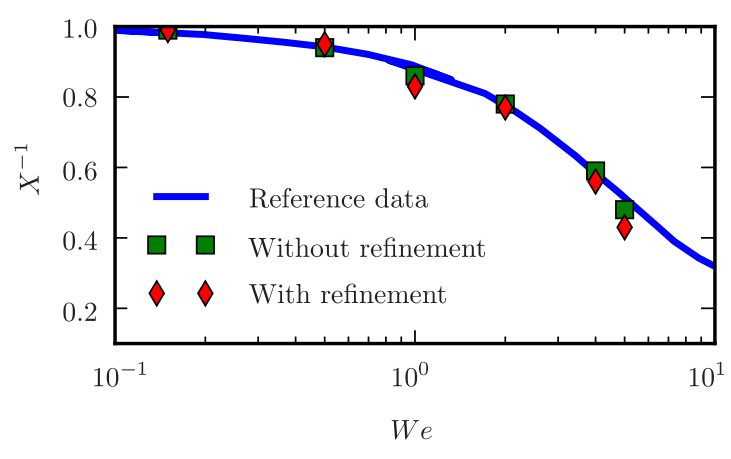

Figure 6.5: (a) Snapshots of a drop immersed in a cross flow at two time instants; top and bottom panel corresponds to its initial and deformed state, respectively. (b) Comparison of the inverse of the mean aspect ratio of the drop versus the Weber number with and without the refinement technique. The reference data is given by a fit from several experimental measurements given in [163].

\begin{tabular}{c|c|c}
$W e$ & Base $N_{f}$ & Refined $N_{f}$ \\
\hline \hline 0.15 & 162 & 324 \\
0.50 & 162 & 628 \\
1.00 & 642 & 1926 \\
2.00 & 642 & 2568 \\
4.00 & 2562 & 7686 \\
5,00 & 2562 & 10248 \\
\hline
\end{tabular}

Table 6.1: Details on the base mesh and refined mesh of the Lagrangian surface for the simulations shown in figure 6.5 
boundary layer development on the surface of the drop leads to its deformation. We adopt a interaction potential based deformation approach geared towards liquid-liquid interfaces as has been described in detail by [167]. The computational domain is taken to be of size $L=(10,5,5) d_{\mathrm{eq}}, d_{\mathrm{eq}}$ is the diameter of the drop in its initial spherical shape. The details on the base resolution and refined resolution of the immersed sphere is given in table 6.1. The spherical drop is placed at $\boldsymbol{x}=(0.5,0.5,0.5) L_{z}$ and the vertical direction $\left(\hat{e}_{z}\right)$ is wall bounded with stationary free-slip walls; $\hat{e}_{y}$ direction is periodic in nature and a uniform flow of $\boldsymbol{U}=U \hat{e}_{x}$ is imposed in the $\hat{e}_{x}$ direction. In figure 6.5(a) we show the morphology of the drop for $W e=1.0$ at two different time instants showing its initial spherical state and deformed state. To quantify the shape of the immersed drop we compute the mean aspect ratio of the drop as $X=l_{z} / l_{x}$ where $l_{z}$ and $l_{x}$ are the lengths of a box surrounding the drop in $\hat{e}_{z}, \hat{e}_{x}$ directions, respectively. In figure $6.5(\mathrm{~b})$, we compare the inverse of the mean aspect ratio versus the Weber number for drops using the refinement technique with the simulations without using refinement and also with reference data taken from experimental measurements and a good agreement between all three data-sets is observed. This shows that the adaptive refinement technique can be used successfully to decouple the deformation mesh from the IBM mesh.

\subsection{Coarse-grained collision detection}

The basic idea of the algorithm is as follows. A collision event occurs when any two arbitrary Lagrangian markers belonging to two separate immersed bodies occupy the same Eulerian cell. This assumption is reasonable since within the immersed boundary framework the thin film fluid dynamics between two bodies which are just about to collide can never be resolved [170]. Once it is determined that two markers belonging to separate bodies are occupying the same Eulerian cell, a collision force is activated on the respective Lagrangian markers along the normal to the surface of the immersed body. Here it is important to note that the algorithm in particularly useful and computationally efficient when there are several dispersed bodies in the flow which can interact with each other and the surrounding fluid simultaneously.

First, we initialise a three dimensional integer array $\operatorname{col} 1[1: N 1,1: N 2,1: N 3]=0$, where $\mathrm{N} 1, \mathrm{~N} 2, \mathrm{~N} 3$ are the number of Eulerian grid points in each direction. This array can have three states; $\operatorname{coll}[i, j, k]=0$ implies there is no Lagrangian marker occupying the cell, $\operatorname{coll}[i, j, k]>0$ implies there are one or more Lagrangian markers belonging to the same immersed body in the cell, $\operatorname{col} l[i, j, k]=-1$ implies markers from different immersed bodies occupy the cell and a collision event must occurs in 
(a)

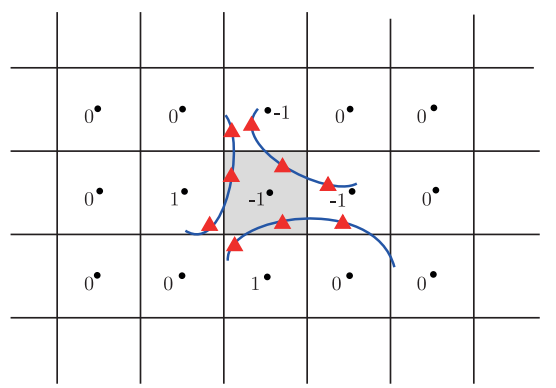

(b)

$$
\begin{aligned}
& \text { Surface of the immersed body } \\
& \text { Lagrangian marker }
\end{aligned}
$$

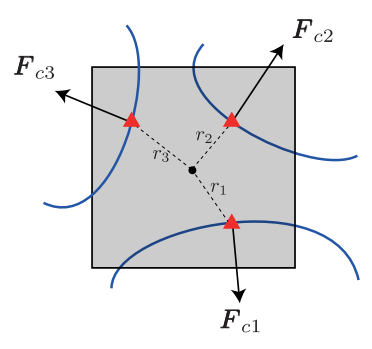

\begin{tabular}{|c|c|c|c|}
\hline 1 & 2 & 3 & 4 \\
\hline 5 & 6 & 7 & 8 \\
\hline
\end{tabular}

- Eulerian cell centre

(c)

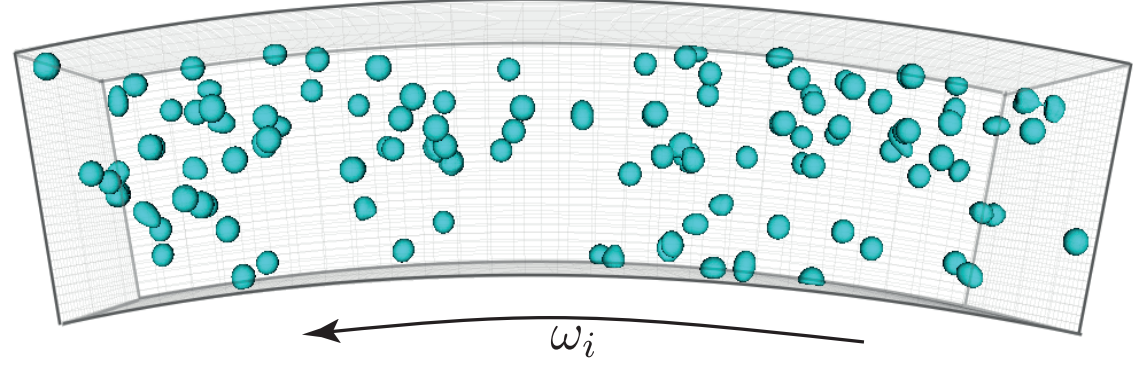

Figure 6.6: (a) A schematic of the grid showing the values of the array coll with three different deformable bodies approaching each other in the darkened Eulerian cell. (b) Snapshots at different time instants of a deformable drop (shown in red) driven by a cross flow colliding with a stationary rigid sphere (shown in blue). (c) Instantaneous snapshot of 120 deformable drops interacting with the wall and among themselves in a laminar Taylor-Couette flow. 
this cell. In figure 6.6(a) we show a schematic of an Eulerian grid with three immersed bodies occupying the same Eulerian cell i.e. the value of the array coll in this cell is set to -1 . The values of the array coll is shown next the respective Eulerian cell centres in figure 6.6(a). In such a situation a collision force is activated on each of these markers. For the simulations shown here, we assume that the magnitude of the collision force is inversely proportional to the distance between the Lagrangian marker and the Eulerian centre. The formulation of the force can be of various types where lubrication forces can be included based on the approach velocity of the marker with respect to the Eulerian cell centre; however, the focus here is on the more general problem of detecting a collision event. We now present a pseudo-code for the algorithm which shows its ease of implementation into any general Navier-Stokes solver.

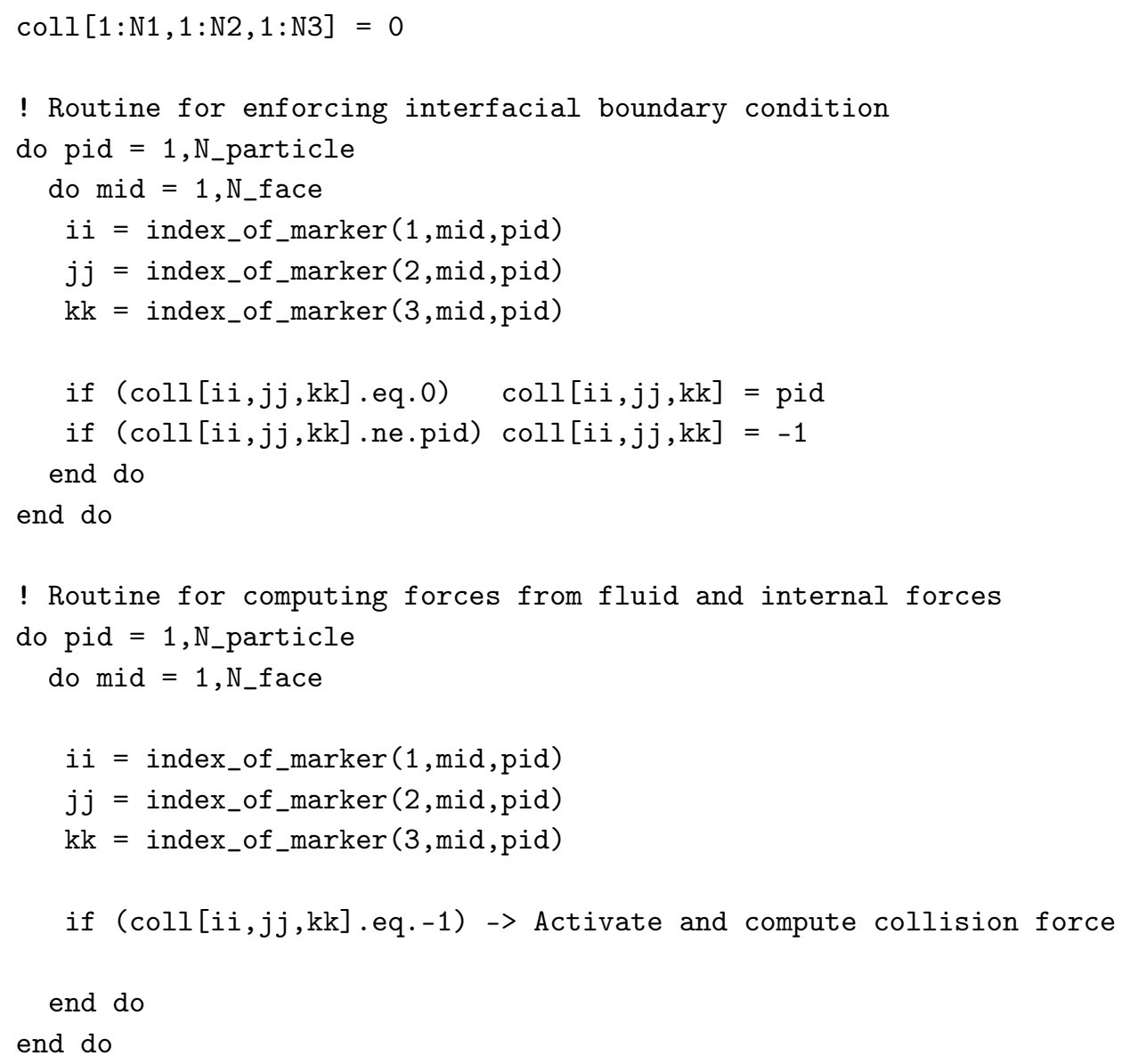


In figure 6.6(b), we show snapshots at different time instants of simulation where a freely moving drop driven by a cross flow collides with a stationary rigid sphere. As can be seen, the collision event occurs smoothly without both bodies penetrating each other. In the supplementary material we have attached an animation which shows collision events between 120 deforming drops with 2562 markers each immersed in a laminar Taylor-Couette flow. An instantaneous snapshot of this animation is shown in figure 6.6(c). Here it is important to emphasize that the overhead in the overall simulation time on implementing the collision-detection algorithm described above is less than $2 \%$ in comparison to other more fine-grained techniques which have a minimum overhead of at least $10 \%$ for such large scale simulations.

\subsection{Summary}

The immersed boundary method (IBM) is becoming a popular tool among computational scientists to tackle problems involving moving and deformable bodies immersed in a fluid. While IBM provides a simple and efficient computational framework for such problems, there are certain inherent issues which limits the scale of the systems that can be studied. For example, current state of art simulations can handle $O\left(10^{2}\right)$ dispersed deformable bodies in weakly turbulent flows. In this chapter, we propose and test different algorithms which can be used to handle $O\left(10^{4}\right)$ dispersed deformable bodies in highly turbulent flows within the immersed boundary framework. We describe a fast moving least squares approximation technique where the transfer functions used to exchange information between the Eulerian and Lagrangian meshes are computed only once during the start of the simulation and a computationally inexpensive interpolation technique is used during the simulation to estimate the transfer function at any arbitrary point in the domain. We find that with sufficient transfer functions stored at the start of the simulation, we are not only able to estimate the transfer functions within acceptable levels of accuracy but also reduce drastically the total computational time required with increasing immersed Lagrangian markers. We also show that through a systematic refinement of the Lagrangian mesh, it can be decoupled into two meshes; a base mesh which can be used for the solution of the deformation governing equations and a refined mesh which is used to enforce the interfacial boundary condition. Additionally, such a procedure can also be used when the dispersed bodies are immersed in flows with stretched Eulerian grids thus eliminating redundant Lagrangian nodes. Finally, we present a simple and easy to implement algorithm to detect collision events between Lagrangian markers residing on different deformable bodies with a computational overhead of less than $2 \%$ for any 
number of Lagrangian markers immersed in the flow. 
COARSE GRAINED ALGORITHMS FOR THE IMMERSED BOUNDARY METHOD 
Chapter 7

Finite-size bubbles and drops in Taylor-Couette flow 


\subsection{Introduction}

In $\S 2$, we studied the effect of sub-Kolmogorov spherical bubbles on the overall drag reduction that can be achieved in Taylor-Couette (TC) flow. In $\S 3$ and $\S 4$ we improved the Euler-Lagrangian tracking used in $\S 2$ by coupling it with a sub-grid deformation model which was then used to study the deformation and orientation statistics of deformable sub-Kolmogorov bubbles and drops dispersed into TC flow. More importantly, we studied the effect of deformation of sub-Kolmogorov bubbles on the overall drag reduction that can be attained in $\mathrm{TC}$ flow. The techniques and algorithms used in $\S 2, \S 3$ and $\S 4$ limit the size of the dispersed bubbles or drops to be of the same scale or smaller than the Kolmogorov length scale. In reality, the dispersed bubbles or drops can be much larger than the Kolmogorov length scale and to tackle this problem we have discussed simple and efficient algorithms in $\S 5$ and $\S 6$ which can be used to simulate finite-size deformable bubbles or drops in a surrounding turbulent flow field. In this chapter, we use the algorithms introduced in $\S 5$ and $\S 6$ to study the influence of finite-size bubbles and drops on the carrier-phase dynamics in $\mathrm{TC}$ flow.

As has been discussed extensively in previous chapters, the main motivation in understanding the dynamics of bubbles and drops in TC flow is to understand the exact physical mechanism behind the reduction in skin-friction that has been observed in both experiments and numerical simulations. Given the wide range of governing parameters and flow regimes in TC flow, it would be extremely challenging to carefully isolate the effect of each parameter without the combined effort of high precision experiments and numerical simulations. The experiments by Murai et al. [18, 19] clearly showed that in the low Reynolds number regime $\left(R e_{i}=1000-2500\right)$ of TC flow, where coherent structures play an active role, injection of $0.1 \%$ of bubbles in silicone oil can reduce the driving torque on the rotating cylinder up to $25 \%$. In the highly turbulent regime $\left(R e_{i}=10^{5}-10^{6}\right)$, where both boundary layers and bulk become fully turbulent, van den Berg et al. [20, 21] demonstrated similar drag reduction behaviour in TC flow. Recent experimental studies on two-phase TC flow in the highly turbulent regime show that the extent of deformability of the dispersed bubbles is crucial to achieve 'strong' drag reduction effects [4, 22]. Here, the word 'strong' implies $40 \%$ drag reduction with just $4 \%$ volume fraction of bubbles injected into the flow. Numerical simulations on finite-size bubbles in vertical channel flows have also shown that deformability of bubbles is crucial for achieving drag reduction in a two-phase turbulent flow [12]. The drag reduction induced by bubbles in channel flows is primarily related to differences in the preferential 
accumulation of the dispersed phase. However, the experiments of van Gils et al. [4] indicate that in TC flow, strong drag reduction need not necessarily be related to any preferential accumulation of the bubbles. This indicates that there exists differences in the fundamental physical mechanisms behind drag reduction induced by finite-size bubbles in TC flow and channel flows which sets up the motivation for this chapter.

Detailed numerical simulations are a necessity when attempting to understand the underlying physics behind drag reduction in different flow regimes. In this chapter, we will focus on understanding the influence of bubbles and drops larger than the Kolmogorov scale $\left(d_{b} / \eta_{K} \sim 20-30\right)$ on the carrier fluid dynamics in TC flow. Here it is important to note that the objective of this chapter is not to mimic the experiments of van Gils et al. which operate at extremely high Reynolds numbers $\left(R e_{i}=10^{5}-10^{6}\right)$ with deformable bubbles which can breakup and coalesce. Such systems are out of reach of numerical simulations even with the algorithms discussed in previous chapters. On the other hand, we focus on understanding from a quantitative point of view the effect of deformable bubbles and drops on the carrier phase dynamics in similar flow regimes. In particular, what would be effect of finite-size bubbles and drops on the drag of the rotating cylinder? In case of drag modification (reduction or enhancement) what would be the physical mechanisms underlying it? Is deformability indeed the crucial factor in achieving strong drag reduction? These are some of the questions we will probe in this chapter.

\subsection{Numerical details}

The dynamics of the carrier phase is computed by solving the Navier-Stokes equations in cylindrical coordinates; refer to equations 1.1 and 1.2 and the corresponding numerical details in $\S 1$. The influence of a dispersed phase (bubbles or drops) on the surrounding fluid is achieved using an immersed boundary method while the deformation of the bubbles and drops is achieved through a phenomenological interaction potential approach. Both the immersed boundary method and the interaction potential approach have been described in detail in $\S 5$.

As discussed in $\S 1$, there can be several governing parameters in a multiphase flow; for example density ratio $\left(\hat{\rho}=\rho_{p} / \rho_{f}\right)$, viscosity ratio $\left(\hat{\mu}=\mu_{p} / \mu_{f}\right)$, Froude number $F r$, Capillary number $(C a)$, Weber number $(W e)$, volume fraction $\left(\alpha_{g}\right)$, interfacial boundary condition (no-slip or free-slip) etc. In this chapter, we focus on the effect on in $\S 2$.

Differences exist also in the case of sub-Kolmogorov bubbles and this has been discussed briefly 
the effect of Weber number $W e$ which quantifies the ratio between the inertial forces to the surface tension forces acting on the immersed bubbles or drops and is typically written as $W e=\rho_{f} U_{\text {ref }}^{2} d_{\mathrm{eq}} / \sigma$. The choice of the reference velocity in the definition of $W e$ varies from one study to the other; for example in the experiments by van Gils et al. [4] the reference velocity was taken to be the liquid velocity fluctuation at the position of the bubble. Here, we use the approach described in $\S 5$, and take the reference velocity to be the velocity of the inner cylinder. In both cases, the main idea is that the Weber number indicates the extent of deformability of the dispersed phase; i.e. high value of $W e$ implies a deformable bubble/drop while low $W e$ implies the bubble/drop is spherical and difficult to deform. We consider both bubbles and drops injected into TC flow; for bubbles $\hat{\rho}=0.01$ and $\hat{\mu} \sim 0$ while for drops $\hat{\rho}=1$ and $\hat{\mu}=1$. Since our Navier-Stokes solver does not allow for viscosity variation within the flow domain, we are restricted by the choice of the viscosity ratio i.e. the algorithms can only handle systems either with $\hat{\mu} \sim 0$ or $\hat{\mu}=1$. The reasons and consequences of this constraint have been discussed in $\S 5$. The interfacial boundary condition for both bubbles and drops is taken to be no-slip no-penetration. While we have the flexibility for simulations with a free-slip no-penetration boundary conditions, it is not the focus of this chapter. The radius ratio of the TC set up is chosen to be $\eta=r_{i} / r_{o}=0.909$ while the aspect ratio is set to $L /\left(r_{o}-r_{i}\right)=2$. We will first consider a Reynolds number of $R e_{i}=5 \times 10^{3}$ which corresponds to the 'low' Reynolds number regime where the coherent structure still play an important role in the momentum transport.

Before analysing the results on the drag modification induced by finite-size bubbles and drops, a comment is necessary regarding the quantification of drag in a two-phase flow simulated within the immersed boundary framework implemented in a Navier-Stokes solver described in $§ 1$. Typically, torque required to drive the inner cylinder in TC flow at a specific speed is directly related to the energy injected into the flow. One way to quantify the torque on the driving cylinder is through the Nusselt number as defined in $\S 2$. However, a similar approach cannot be used in simulations involving bubbles $(\hat{\mu} \sim 0)$ represented through the immersed boundary method (IBM) in a Navier-Stokes solver which does not allow for viscosity gradients in the flow domain. While the effect of a buoyant body can be easily replicated in the simulation, doing the same for viscosity is not straight-forward. Any fluid motion inside an immersed body (bubble or a drop) with viscosity different from that of the surrounding fluid is artificial and should not be considered while trying to understand the flow physics or computing the statistics.

To understand the consequences of this constraint more clearly, in figure 7.1 we show a schematic of a fluid confined in between the inner cylinder (IC) and the outer 


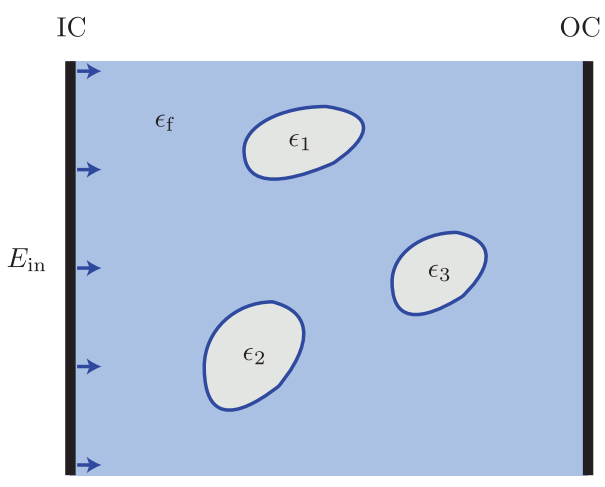

Figure 7.1: A schematic of energy input $\left(E_{\text {in }}\right)$ from the inner cylinder and dissipation inside the fluid $\left(\epsilon_{f}\right)$ and the dispersed phase $\left(\epsilon_{1}, \epsilon_{2} \ldots\right)$.

cylinder (OC) with three finite size bubbles dispersed in the gap. The energy injected into the flow by driving the inner cylinder is represented by $E_{\mathrm{in}}$. In a single phase flow, once statistical stationarity is achieved there is a balance between the energy injected $E_{\text {in }}$ and the dissipation in the fluid $\epsilon_{\mathrm{f}}$. In the case of multiphase flows with dispersed bubbles or drops, dissipation also occurs inside the dispersed phase as represented by $\epsilon_{i}$ in figure 7.1. In the case of bubbles, where the viscosity ratio $\hat{\mu}=\mu_{p} / \mu_{f} \sim 0$ the dissipation inside the dispersed phase is negligible. However, since the viscosity of the dispersed phase is equal to the viscosity of the surrounding fluid, the dissipation inside the bubble in the simulation itself is finite. Thus, the energy that is given as input from the inner cylinder to the fluid mixture as shown in figure 7.1 is distributed both into the dissipation in the fluid and the dissipation of the artificial fluid bounded by the interface. This needs to be taken into account while computing the statistics in a two-phase flow simulated using IBM. In simulations involving bubbles dispersed in TC flow we will compute the dissipation in the fluid $\left(\epsilon_{\mathrm{f}}\right)$ and compare this with the mean dissipation in the corresponding single phase flow. The net drag reduction is then computed as $D R=100\left(\epsilon_{s}-\epsilon_{t}\right) / \epsilon_{s}$. Here it is important to realise that the mean dissipation but not torque on the inner cylinder is used to compute the drag reduction.

\subsection{Results}

In figure 7.2, we show drag reduction versus the Weber number for both bubbles and drops injected into TC flow at $R e_{i}=5000$. One can easily observe that in the 


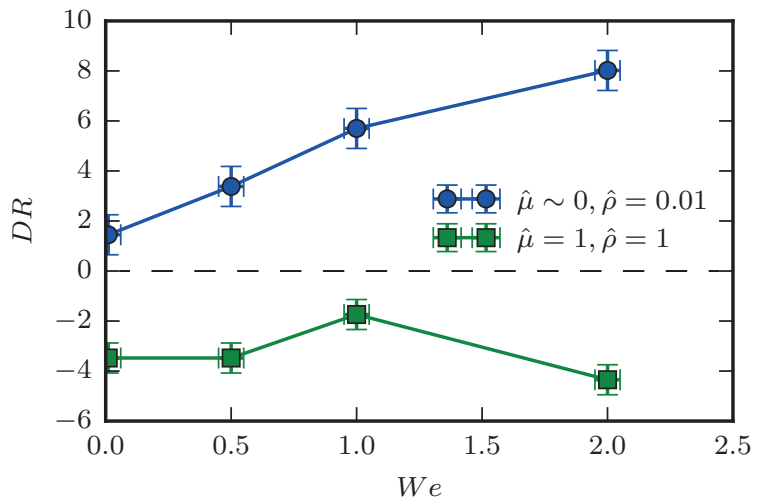

Figure 7.2: Drag reduction versus the Weber number for bubbles $(\hat{\mu} \sim 0, \hat{\rho}=0.01)$ and drops $(\hat{\mu}=1, \hat{\rho}=1)$ for inner cylinder Reynolds number of $R e_{i}=5000$; volume fraction of the bubbles $\alpha_{g} \sim 1 \%$

case of bubbles injected into the flow the net drag reduction increases with increasing Weber number. In other words, we observe that increasing the deformability of the bubbles reduces the mean dissipation in the surrounding fluid in comparison to single phase flow. At very low Weber numbers, when the bubbles are close to spherical and non-deformable, negligible drag reduction is observed. In the case of drops, negative drag reduction is observed irrespective of the extent of deformability i.e. the mean dissipation in the fluid is enhanced in comparison to the single phase flow. Here it is important to note that in the case of neutrally buoyant drops with similar viscosity the net drag reduction can be computed both from the torques on the cylinder and also the mean dissipation in the system.

Let us now focus on understanding the cause behind drag reduction attained in the case of bubbles injected into the flow. In figure 7.3, we plot the local bubble volume fraction averaged azimuthally, axially and over time versus the normalised wall-normal position $\tilde{r}=\left(r-r_{i}\right) / d$. There is no observable difference in the accumulation patterns of the bubbles for different Weber numbers. In all cases, the bubbles prefer to position themselves near the inner cylinder due to the radially inwards favourable pressure gradient in TC flow. From figure 7.3, we can conclude that the increase in drag reduction observed for bubbles in figure 7.2 is not related to any preferential accumulation of the bubbles in the cylinder gap. A similar observation was made in the experiments of van Gils et al. [4] where it was shown that a higher local gas concentration near the inner wall does not guarantee strong drag reduction. Instead, 


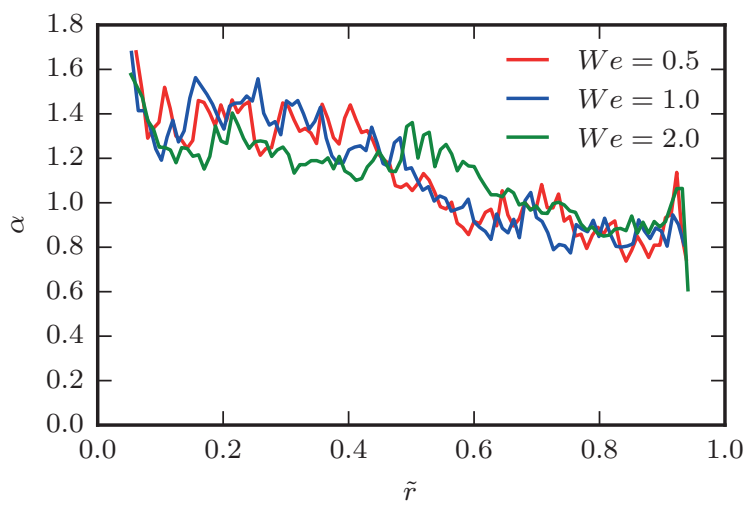

Figure 7.3: Radial profiles of local bubble volume fraction for different Weber numbers at $R e_{i}=5000$. The global volume fraction is $\alpha_{g} \sim 1 \%$

experimental measurements show that a higher gas volume fraction near the inner cylinder leads to moderate drag reduction. The main idea to take away here is that the increase in drag reduction need not be necessarily related to bubble accumulation patterns in the cylinder gap. Here, it is also worth referring to $\S 4$, where we studied the the effect of sub-Kolmogorov deformable bubbles on the drag reduction and it was shown that bubble accumulation patterns are crucial for increasing drag reduction. This indicates that there are differences not only between channel flows and TC flow as discussed previously but also in the case of sub-Kolmogorov and finite-size bubbles.

To understand the flow state of the carrier fluid, we look at the angular velocity transport from the inner cylinder to the outer cylinder. In single phase flow, the angular velocity flux is conserved along the wall-normal position i.e. $\partial_{r}\left[r^{3}\left\langle u_{r} \omega\right\rangle-r^{3} \nu \partial_{r}\langle\omega\rangle\right]=\partial_{r}\left[J_{\omega}\right]=0$. In two-phase flows, due to the forcing from the dispersed phase, this expression is corrected accordingly and is written as $\partial_{r}\left[r^{3}\left\langle u_{r} \omega\right\rangle-r^{3} \nu \partial_{r}\langle\omega\rangle\right]+r^{2} f_{\theta}=\partial_{r}\left[J_{\omega}\right]+F_{\theta}=0$. In these expressions, $J_{\omega}$ is the angular velocity flux, $\omega$ is the angular velocity, $r$ is the wall-normal position, $\langle\ldots\rangle$ refers to averaging in the homogeneous directions (azimuthal and axial) and over time and $f_{\theta}$ is the forcing from the dispersed phase onto the carrier fluid in the azimuthal direction. To analyse the stresses that are transported from the inner cylinder to the outer cylinder, we plot the azimuthally, axially and time averaged value of $J_{\omega}$ normalised with its corresponding laminar value $J_{\text {lam }}$ versus the normalised radial position in figure 7.4 .

As mentioned earlier, in single phase flow, $J_{\omega}$ is constant along the radial position 


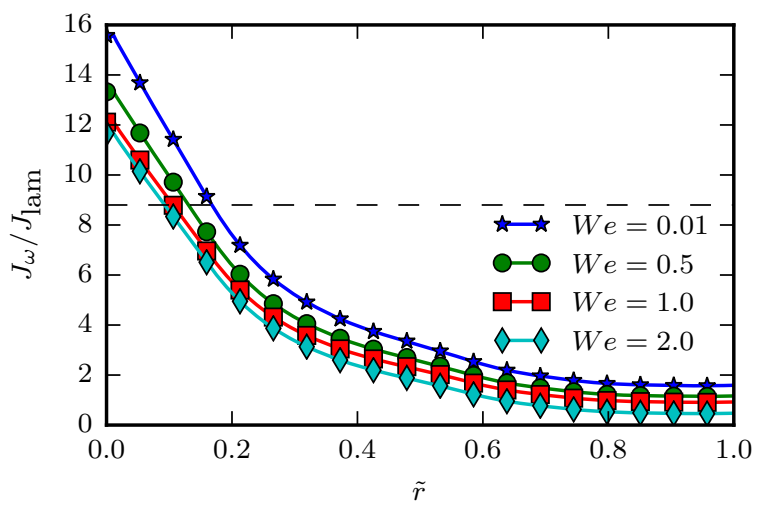

Figure 7.4: Radial profiles of angular velocity transport for different Weber numbers for $R e_{i}=5000$. The profiles are scaled according to the true dissipation computed only in the nodes occupied by the fluid.

and for $R e_{i}=5000$ this is shown by a dotted line in figure 7.4. In the case of two-phase flow, the value $J_{\omega}$ is higher than that of single phase flow close to the inner cylinder and relatively lower in the bulk and near the outer cylinder. A lower value of $J_{\omega}$ in comparison to the single phase case indicates an effective laminarisation of the flow i.e. the bubbles reduce the radial and axial velocity fluctuations and in turn the Reynolds stresses. Close to the inner cylinder, $J_{\omega}$ is much higher than that of a single phase flow and this is due to the dissipation induced by the collision of the bubbles with the inner cylinder. Furthermore, the wake of the bubbles are also regions of intense dissipation.

In figure 7.5, we show instantaneous pseudo colour plots of azimuthal velocity for the single phase case (left panel) and the two-phase case with $W e=1.0$ (right panel). One can clearly observe the ejection of plumes in the flow without bubbles and also the corresponding Taylor rolls. However, in the presence of bubbles, the plume ejections are fully blocked near the inner cylinder and the flow in the bulk remains relatively less turbulent. A footprint of the bubbles is also seen in the snapshot for two-phase flow. A more intuitive way of studying the effect of bubbles on the plume ejections and Taylor rolls is to look at dissipation in the flow. In figure 7.6, we show contours of dissipation in the flow with and without bubbles. In the case of single phase flow, there is intense dissipation near the walls and around the plumes which are injected into the bulk and form the Taylor rolls. Injection of bubbles (right panel of figure 7.6) completely kills the plumes and laminarises the bulk. Another 

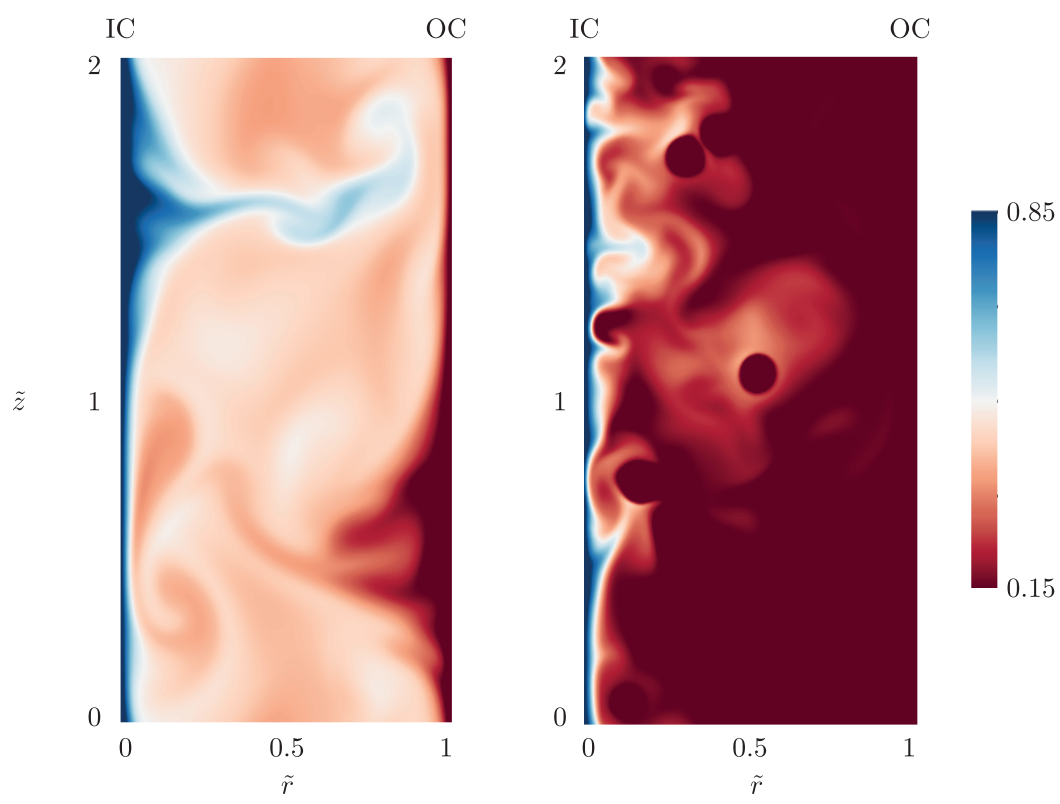

Figure 7.5: Instantaneous snapshot of azimuthal velocity for single phase (left panel) and two-phase flow with $W e=1.0$ (right panel) at $R e_{i}=5000$. 


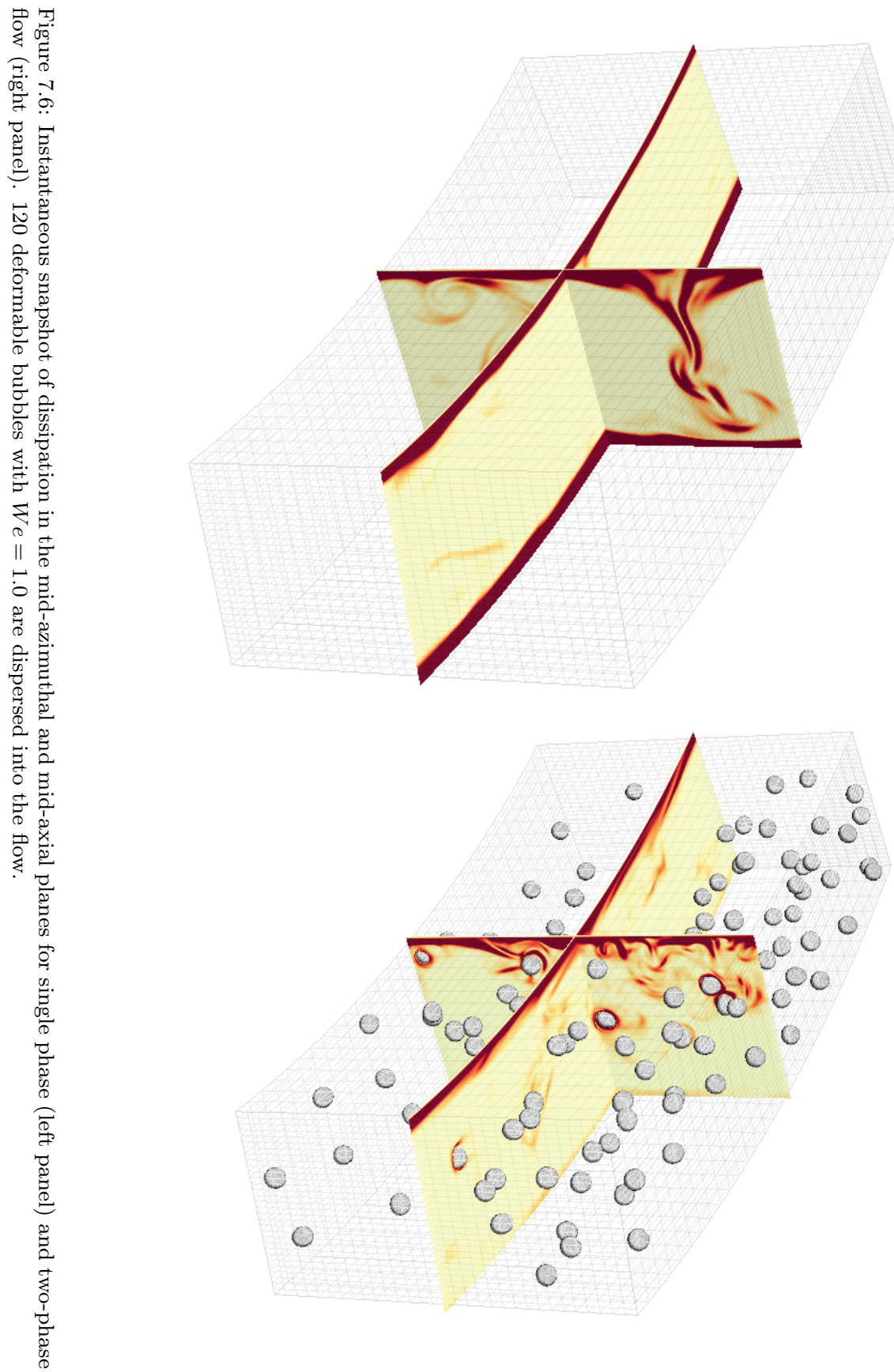


interesting observation that can be made from figure 7.6 which resonates very well with the ideas discussed above is the extent of dissipation near the outer cylinder. There is considerable dissipation near the outer wall in single phase flow, while due to laminarisation relatively lower dissipation is seen in the case of two-phase flow.

The mechanism of attaining drag reduction by blocking the formation of plumes into the bulk is similar to what has been observed in the case of sub-Kolmogorov bubbles (§2). However, what is observed in the case of finite-size bubbles is that while both low and high $W e$ bubbles seem to disrupt the formation of plumes and laminarise the bulk, only high $W e$ number bubbles produce drag reduction. This is because the deformable bubbles (high $W e$ ) have much weaker wake in the direction they prefer to align i.e. the stream-wise direction and this results in an overall lower dissipation in the wake in comparison to spherical bubbles. One way to quantify this effect would be to study the instantaneous bubble Reynolds numbers near the inner cylinder which can be computed as $R e_{b}=|\mathbf{u}-\mathbf{v}| d_{p} / \nu$; where $\mathbf{u}$ and $\mathbf{v}$ are the local fluid velocity and bubble centre of mass velocity, respectively. Computing the local fluid velocity $\mathbf{u}$ is a delicate issue within the IBM framework since the fluid nodes next to the interface are forced. Different methods of evaluating the fluid velocity are currently being tested. Another aspect that is being explored is the effect of bubbles in the highly turbulent regime i.e. when the boundary layers become turbulent. Preliminary results show that similar trends in drag reduction are observed and the deformability of bubbles blocks the fluctuations in the radial velocity from the inner cylinder rather than the coherent plumes in this regime. As expected, these simulations converge at a relatively slower rate and are currently in progress.

\subsection{Summary}

In this chapter, we have studied the effect of finite size bubbles and drops on the net drag reduction that can be achieved in Taylor-Couette flow. We focus on understanding the effect of deformability of the injected bubbles and drops (quantified by the Weber number $W e$ ) on the carrier fluid. In the case of bubbles simulated using the immersed boundary method within a Navier-Stokes solver which does not allow for viscosity variation in the flow domain, we have discussed the need to quantify drag in terms of the mean dissipation in the fluid and not torque on the cylinders. This constraint is relaxed in the case of neutrally buoyant drops with similar viscosity, where there is no difference between computing the mean dissipation in the system and torque on the cylinders. Our simulations show that in the case of bubbles, the net drag reduction increases with increasing deformability while injecting neutrally 
buoyant drops leads to drag enhancement. More importantly, the increase in drag reduction with increasing deformability in the case of bubbles is not related to any preferential accumulation in the cylinder gap. We have shown quantitatively that finite-size bubbles block the transfer of momentum from the inner cylinder to the outer cylinder thus laminarising the bulk and effectively leading to drag reduction. While both rigid and deformable bubbles block the formation of plumes, the drag reduction attained by the bubbles is reduced by the dissipation in the wake. This dissipation is much stronger for the case of spherical bubbles in comparison to deformable bubbles thus leading to negligible drag reduction. The effect of the bubbles and drops on turbulent boundary layers is currently being explored. 
Chapter 8

Conclusions 
In this thesis, we have studied the behaviour of bubbles and drops in a turbulent Taylor-Couette (TC) flow through the combination of a variety of numerical tools. In particular, we have focussed on understanding the effect of injecting bubbles and drops on the overall drag in the system while developing new numerical tools to access different flow regimes.

In $\S 2$ we use a two-way coupled Euler-Lagrange approach to study the effect of sub-Kolmogorov bubbles on the overall friction on the driving cylinder in TC flow. The Reynolds number of the flow is limited to $R e_{i}=8000$ which allows us to focus on the interplay between the dispersed bubbles and the coherent Taylor rolls which dominate momentum transport in this regime. More importantly, we focus on independent control of the Reynolds number and the relative strength of the buoyancy of the bubbles as compared to the inner cylinder driving (quantified by the Froude number $\mathrm{Fr}$ ) which is not possible in experiments. We find that drag reduction is strongly related to the Froude number: for fixed Reynolds number we observe higher drag reduction when $F r<1$ than $F r>1$. This buoyancy-induced drag reduction effect is more prominent in low $R e_{i}$ systems and decreases with increasing Reynolds number $R e_{i}$. We trace the cause for drag reduction back to the weakening of the Taylor rolls by the rising bubbles. The weakening of the Taylor rolls is closely related to the motion of the bubbles near the plume ejection regions of the inner cylinder. By analysing individual trajectories of bubbles, we found that for $R e_{i}=2500$ the bubbles have primarily either upward motion (lower $F r$ ) or coherent motion inside the Taylor roll (larger $\mathrm{Fr}$ ) which essentially dictates the overall impact of the bubbles on the plumes. Similar behaviour was observed even in the case of a higher $R e_{i}$, but with a more erratic movement within the underlying turbulent vortices which explains the consistent drop in drag reduction with increasing Reynolds number.

Point-particle Euler-Lagrange type techniques (used in §2) allows us to simulate $O\left(10^{5}\right)$ bubbles, drops or particles in a given flow. However, it places severe constraints on the properties of the dispersed phase, i.e. the dispersed phase has to be smaller than the Kolmogorov scale in the flow and have a fixed shape (spherical or ellipsoidal) throughout the numerical simulation. While the constraint on the size of the dispersed phase is ingrained into the formulation of point-particle type techniques, the constraint on having a fixed shape through out the simulation is not rigid. Keeping this in mind, in $\S 3$ and $\S 4$, we couple a phenomenological sub-grid deformation model with Euler-Lagrangian tracking which allows us to simulate $O\left(10^{5}\right)$ deformable sub-Kolmogorov bubbles or drops dispersed in a time varying turbulent flow field. 
In $\S 3$, we focus on understanding the influence of the capillary number $C a$ (ratio of viscous stretching forces and surface tension forces) and viscosity ratio $(\hat{\mu})$ of the dispersed phase and the carrier phase on the deformation and orientation statistics of neutrally buoyant sub-Kolmogorov drops dispersed into TC flow. Given the sub-Kolmogorov and neutrally-buoyant nature of the drops, they are advected as passive tracers in the turbulent flow field. In order to account for deformability and orientation, the dispersed drops are considered to be tri-axial ellipsoids which allows us to mathematically represent the surface of the drop using a symmetric second-order positive definite tensor. Increasing the capillary number results in large deformations of the dispersed droplets due to weakening surface tension forces. Additionally, the drops deform more when they are in the inner and outer boundary layer as compared to the bulk region. Due to increased influence of rotation with increase in viscosity ratio, the droplets tend to be more oblate or disk-like as compared to more prolate or cigar-like droplets for low viscosity ratio systems. Regardless of the capillary number $C a$ and viscosity ratio $\hat{\mu}$, the major axis of the drops tends to align with the stream-wise direction in the inner and outer boundary layer while the orientation is highly isotropic in the bulk; a behaviour similar to solid ellipsoidal particles in a turbulent channel flow. However, the preference of orientation with the eigen directions of strain rate tensor of these drops is very different from those exhibited by axis-symmetric ellipsoids in a isotropic turbulent flow.

In order to study the motion and influence of sub-Kolmogorov deformable bubbles, we couple the sub-grid deformation model with a momentum equation for the dispersed phase in $\S 4$. Inclusion of two-way coupling allows us to study not only the deformation and orientation characteristics of the dispersed bubbles but also its effect on the overall drag reduction in TC flow. The simulations performed using this approach are currently impossible with fully-resolved techniques due to the sub-Kolmogorov nature of the dispersed phase. We find that at an inner cylinder Reynolds number of $R e_{i}=2500$, increasing the deformability (i.e. the capillary number) results in an increase in the overall drag reduction. By looking at the mean bubble volume fraction profiles in the domain, we find that in comparison to spherical bubbles, deformable bubbles prefer to accumulate more near the rotating inner cylinder. The PDF's of the ellipsoid axes and their orientational preference indicate that near the inner cylinder the bubbles are primarily prolate and tend to align with the stream-wise direction. This results in an increase in the drag experienced by the near-wall deformed bubbles in the wall-normal direction which ultimately results in their accumulation near the inner cylinder. Drag on the inner cylinder is directly connected to the strength of plume ejections which are disrupted 
more effectively by deformable bubbles due to their preferential accumulation. At a relatively higher Reynolds number of $R e_{i}=8000$ we find that deformability of sub-Kolmogorov bubbles has no effect on drag reduction as the turbulent fluctuations are much stronger in comparison to the buoyancy of the bubbles. At this Reynolds number and beyond, i.e. when both boundary layers and bulk become turbulent, experiments have shown that finite-size (larger than Kolmogorov scale) bubbles which can deform, produce strong drag reduction effects in spite of no recognisable difference in their accumulation near the driving wall [4]. This indicates that the drag reduction induced by finite-size bubbles at very high Reynolds numbers $\left(\operatorname{Re} \sim 10^{5}-10^{6}\right)$ is fundamentally different from what has been described in $\S 4$ which is more close to the works of $[18,19]$ where $R e_{i} \sim 10^{3}$. Additionally, in the case of finite-size bubbles, inertial forces become relevant in the deformation dynamics and this makes the Weber number an important parameter. Numerical simulations of such systems would invariably require fully resolved simulations with methods such as front-tracking, volume of fluid or immersed-boundaries etc.

In $\S 5$, we have demonstrated the implementation of a finite-difference based flow solver capable of handling several deforming finite-size bubbles or drops where the deformation dynamics is computed through an interaction potential (IP) approach. An immersed boundary method (IBM) based on moving least squares interpolation is used to enforce the interfacial boundary condition on the underlying flow while the deformation dynamics is computed through minimising the potential energy of a spring network spread over the surface of an immersed drop or a bubble. A unique feature of this approach in comparison to conventional techniques used to simulate finite size immersed drops or bubbles is its computational efficiency which allows us to scale up both in terms of the number of drops or bubbles in the flow and also the turbulence levels in the surrounding fluid. By comparing with already existing analytical solutions and experimental measurements of standard configurations (deforming drop in a shear flow and cross flow), we have shown that such an approach is practical, reliable and self-consistent. While the interaction potential approach for deformation is computationally inexpensive, parallelisation is a necessary step to simulate large scale turbulent flows with several thousands of simultaneously deforming bodies. To this effect, we also present a parallelisation strategy which is easy to implement for any generic single phase fluid solver. With pure MPI based parallelisation we have shown that decent scalability is achieved up to $10^{3}$ cores and this is further enhanced to $10^{4}$ cores by including OpenMP directives.

While the algorithms used in the IBM-IP solver presented in $\S 5$ are extremely versatile and easy to implement in any single phase fluid solver, it has certain 
drawbacks which restrict scaling up multiphase simulations. Keeping this in mind, we have introduced multiple algorithms in $\S 6$ which allow us to tackle large scale multiphase flow simulations. We introduce a fast moving least squares approximation technique where the transfer functions used to exchange information between the Eulerian and Lagrangian meshes are computed only once during the start of the simulation and a computationally inexpensive interpolation technique is used during the simulation to estimate the transfer function at any arbitrary point in the domain. We find that with sufficient transfer functions stored at the start of the simulation, we are not only able to estimate the required transfer functions within acceptable levels of accuracy but also reduce drastically the total computational time required with increasing immersed Lagrangian markers. We show that through a systematic refinement of the Lagrangian mesh, it can be decoupled into two meshes; a base mesh which can be used for the solution of the deformation governing equations and a refined mesh which is used to enforce the interfacial boundary condition. Additionally, such a procedure can also be used when the dispersed bodies are immersed in flows with stretched Eulerian grids thus eliminating redundant Lagrangian nodes. We also present a simple and easy to implement algorithm to detect collision events between Lagrangian markers residing on different deformable bodies with a computational overhead of less than $2 \%$ for any number of Lagrangian markers immersed in the flow.

Using the techniques described in $\S 5$ and $\S 6$, we simulate finite-size bubbles and drops in turbulent TC flow in $\S 7$. In particular, we vary the Weber number of the immersed bubbles and drops and look at its influence on the total drag reduction in the system. We find that increasing the deformability of bubbles leads to increase in the net drag reduction in the system while injection of drops leads to drag enhancement. It has been shown quantitatively that finite-size bubbles block the transfer of momentum from the inner cylinder to the outer cylinder thus laminarising the bulk which is the cause for drag reduction. Rigid spherical bubbles produce much stronger wakes in the shear flow system and this overcomes the effect of drag reduction (which is attained by blocking the plume ejections) thus leading to negligible drag reduction in the system. We find similar trends in drag reduction by bubbles in the case of turbulent boundary layers while the effect of deformability on the turbulent fluctuations near the rotating cylinder is currently being explored.

The use of a combination of numerical tools to study the various flow regimes in a multiphase TC flow has been the focal point of this thesis. We have taken a step forward in understanding the underlying physical mechanisms and relevant parameters behind drag reduction induced by bubbles and drops in TC flow. The 
numerical techniques and algorithms developed in this thesis can easily be extended to other flow systems and can also be combined with each other to explore flow regimes that have never been studied before. One such example is the simultaneous simulation of both sub-Kolmogorov and finite-size bubbles and drops within the same flow domain. The algorithms used in $\S 5$ and $\S 6$ can be used to study the dynamics of flexible structures in turbulent flows or even simulate flow of blood inside a full human heart in the coming future. One can confidently state that with the combined effort of high precision experimental measurements and novel numerical tools, the understanding of multiphase flows will continue to grow rapidly in the coming future. 


\section{Bibliography}

[1] O. Darrigol. Worlds of flow: A history of hydrodynamics from the Bernoullis to Prandtl, 2005.

[2] Turbulence. https://en.wikipedia.org/wiki/Turbulence.

[3] O. Reynolds. An experimental investigation of the circumstances which determine whether the motion of water shall be direct or sinuous, and of the law of resistance in parallel channels. Proc. Royal Soc. London, 35(224-226):84-99, 1883.

[4] D.P.M. van Gils, D. Narezo Guzman, C. Sun, and D. Lohse. The importance of bubble deformability for strong drag reduction in bubbly turbulent Taylor-Couette flow. J. Fluid Mech., 722:317-347, 2013.

[5] A. Prosperetti and G. Tryggvason. Computational methods for multiphase flow. Cambridge university press, 2007.

[6] P. Moin and K. Mahesh. Direct numerical simulation: a tool in turbulence research. Annu. Rev. Fluid Mech., 30(1):539-578, 1998.

[7] G. Tryggvason, S. Dabiri, B. Aboulhasanzadeh, and J. Lu. Multiscale considerations in direct numerical simulations of multiphase flows. Phys. Fluids, 25(3):031302, 2013.

[8] S.L. Ceccio. Friction drag reduction of external flows with bubble and gas injection. Annu. Rev. Fluid Mech., 42:183-203, 2010.

[9] N.K. Madavan, S. Deutsch, and C.L. Merkle. Reduction of turbulent skin friction by microbubbles. Technical report, DTIC Document, 1983.

[10] N.K. Madavan, S. Deutsch, and C.L. Merkle. Measurements of local skin friction in a microbubble-modified turbulent boundary layer. J. Fluid Mech., 156:237-256, 1985.

[11] J. Xu, M.R. Maxey, and G.E. Karniadakis. Numerical simulation of turbulent drag reduction using micro-bubbles. J. Fluid Mech., 468:271-281, 2002. 
[12] J. Lu, A. Fernández, and G. Tryggvason. The effect of bubbles on the wall drag in a turbulent channel flow. Phys. Fluids, 17(9):095102, 2005.

[13] Y. Murai, H. Fukuda, Y. Oishi, Y. Kodama, and F. Yamamoto. Skin friction reduction by large air bubbles in a horizontal channel flow. Int. J. Multiphase Flow, 33(2):147-163, 2007.

[14] J. Lu and G. Tryggvason. Effect of bubble deformability in turbulent bubbly upflow in a vertical channel. Phys. Fluids, 20(4):040701, 2008.

[15] S. Dabiri, J. Lu, and G. Tryggvason. Transition between regimes of a vertical channel bubbly upflow due to bubble deformability. Phys. Fluids, 25(10):102110, 2013.

[16] M.J. Pang, J.J. Wei, and B. Yu. Numerical study on modulation of microbubbles on turbulence frictional drag in a horizontal channel. Ocean Engineering, 81:58-68, 2014.

[17] H.J. Park, Y. Tasaka, Y. Oishi, and Y. Murai. Drag reduction promoted by repetitive bubble injection in turbulent channel flows. Int. J. Multiphase Flow, 2015.

[18] Y. Murai, H. Oiwa, and Y. Takeda. Bubble behavior in a vertical Taylor-Couette flow. In J. Physics: Conference Series, volume 14, page 143. IOP Publishing, 2005.

[19] Y. Murai, H. Oiwa, and Y. Takeda. Frictional drag reduction in bubbly Couette-Taylor flow. Phys. Fluids, 20(3):034101, 2008.

[20] T.H. van den Berg, S. Luther, D.P. Lathrop, and D. Lohse. Drag reduction in bubbly Taylor-Couette turbulence. Phys. Rev. Lett., 94(4):044501, 2005.

[21] T.H. van den Berg, D.P.M. van Gils, D.P. Lathrop, and D. Lohse. Bubbly turbulent drag reduction is a boundary layer effect. Phys. Rev. Lett., 98(8):084501, 2007.

[22] R.A. Verschoof, R.C.A. van der Veen, C. Sun, and D. Lohse. Bubble drag reduction requires large bubbles. Phys. Rev. Lett., 117(10):104502, 2016.

[23] Y. Murai. Frictional drag reduction by bubble injection. Exp. Fluids, 55(7):1-28, 2014.

[24] E. Climent, M. Simonnet, and J. Magnaudet. Preferential accumulation of bubbles in Couette-Taylor flow patterns. Phys. Fluids, 19(8):083301, 2007.

[25] A. Chouippe, E. Climent, D. Legendre, and C. Gabillet. Numerical simulation of bubble dispersion in turbulent Taylor-Couette flow. Phys. Fluids, 26(4):043304, 2014.

[26] K. Sugiyama, E. Calzavarini, and D. Lohse. Microbubbly drag reduction in 
Taylor-Couette flow in the wavy vortex regime. J. Fluid Mech., 608:21-41, 2008.

[27] A. Ferrante and S. Elghobashi. On the physical mechanisms of drag reduction in a spatially developing turbulent boundary layer laden with microbubbles. $J$. Fluid Mech., 503:345-355, 2004.

[28] V.S. L'vov, A. Pomyalov, I. Procaccia, and V. Tiberkevich. Drag reduction by microbubbles in turbulent flows: The limit of minute bubbles. Phys. Rev. Lett., 94(17):174502, 2005.

[29] M.M. Rai and P. Moin. Direct simulations of turbulent flow using finite-difference schemes. J. Comp. Phys., 96(1):15-53, 1991.

[30] R. Verzicco and P. Orlandi. A finite-difference scheme for three-dimensional incompressible flows in cylindrical coordinates. J. Comp. Phys., 123(2):402-414, 1996.

[31] E. P. van der Poel, R. Ostilla-Mónico, J. Donners, and R. Verzicco. A pencil distributed finite difference code for strongly turbulent wall-bounded flows. Computers \& Fluids, 116:10-16, 2015.

[32] S. Grossmann, D. Lohse, and C. Sun. High Reynolds number Taylor-Couette turbulence. Annu. Rev. Fluid Mech., 48(1), 2016.

[33] C.D. Andereck, S.S. Liu, and H.L. Swinney. Flow regimes in a circular Couette system with independently rotating cylinders. J. Fluid Mech., 164:155-183, 1986.

[34] R. Ostilla-Mónico, E.P. van der Poel, R. Verzicco, S. Grossmann, and D. Lohse. Exploring the phase diagram of fully turbulent Taylor-Couette flow. J. Fluid Mech., 761:1-26, 2014.

[35] S. B. Pope. Turbulent Flow. Cambridge University Press, 2000.

[36] B. Eckhardt, S. Grossmann, and D. Lohse. Torque scaling in turbulent Taylor-Couette flow between independently rotating cylinders. J. Fluid Mech., 581:221-250, 2007.

[37] K. Yoshida, Y. Tasaka, Y. Murai, and T. Takeda. Mode transition in bubbly Taylor-Couette flow measured by PTV. Jou. Physics: Conference Series, 147(1):012013, 2009.

[38] T. Watamura, Y. Tasaka, and Y. Murai. Intensified and attenuated waves in a microbubble Taylor-Couette flow. Phys. Fluids, 25(5):054107, 2013.

[39] Y. Shiomi, H. Kutsuna, K. Akagawa, and M. Ozawa. Two-phase flow in an annulus with a rotating inner cylinder (flow pattern in bubbly flow region). Nuclear Engineering and Design, 141(1):27-34, 1993. 
[40] H. Djeridi, J-F Fave, J-Y Billard, and D.H. Fruman. Bubble capture and migration in Couette-Taylor flow. Exp. Fluids, 26:233-239, 1999.

[41] H. Djeridi, C. Gabillet, and J.Y. Billard. Two-phase Couette-Taylor flow: Arrangement of the dispersed phase and effects on the flow structures. Phys. Fluids, 16(1):128-139, 2004.

[42] I.M. Mazzitelli, D. Lohse, and F. Toschi. The effect of microbubbles on developed turbulence. Phys. Fluids, 15(1):L5-L8, 2003.

[43] P. Oresta, R. Verzicco, D. Lohse, and A. Prosperetti. Heat transfer mechanisms in bubbly Rayleigh-Bénard convection. Phys. Rev. E, 80(2):026304, 2009.

[44] R. Lakkaraju, F. Toschi, and D. Lohse. Bubbling reduces intermittency in turbulent thermal convection. J. Fluid Mech., 745:1-24, 2014.

[45] J. Magnaudet and I. Eames. The motion of high-reynolds-number bubbles in inhomogeneous flows. Annu. Rev. Fluid Mech., 32(1):659-708, 2000.

[46] T.R. Auton, J.C.R. Hunt, and M. Prud'Homme. The force exerted on a body in inviscid unsteady non-uniform rotational flow. J. Fluid Mech., 197:241-257, 1988.

[47] T.R. Auton. The lift force on a spherical body in a rotational flow. J. Fluid Mech., 183:199-218, 1987.

[48] R. Mei and J.F. Klausner. Unsteady force on a spherical bubble at finite reynolds number with small fluctuations in the free-stream velocity. Phys. Fluids., 4(1):63-70, 1992.

[49] J. Capecelatro and O. Desjardins. An Euler-Lagrange strategy for simulating particle-laden flows. J. Comp. Phys., 238:1-31, 2013.

[50] G.N. Fokoua, C. Gabillet, A. Aubert, and C. Colin. Effect of bubbles arrangement on the viscous torque in bubbly Taylor-Couette flow. Phys. Fluids, 27(3):034105, 2015.

[51] M.J.W. Harleman. On the effect of turbulence on bubbles: experiments and numerical simulations of bubbles in wall-bounded flows. TU Delft, Delft University of Technology, 2012.

[52] J. Jiménez. Cascades in wall-bounded turbulence. Annu. Rev. Fluid Mech., 44(1):27, 2011.

[53] R. Ostilla-Mónico, R.J.A.M. Stevens, S. Grossmann, R. Verzicco, and D. Lohse. Optimal Taylor-Couette flow: direct numerical simulations. J. Fluid Mech., 719:14-46, 2013.

[54] R. Ostilla-Mónico, S.G. Huisman, T.J.G. Jannink, D.P.M. Van Gils, R. Verzicco, S. Grossmann, C. Sun, and D. Lohse. Optimal Taylor-Couette flow: radius ratio dependence. J. Fluid Mech., 747:1-29, 2014. 
[55] R. Ostilla-Mónico, E.P. van der Poel, R. Verzicco, S. Grossmann, and D. Lohse. Boundary layer dynamics at the transition between the classical and the ultimate regime of Taylor-Couette flow. Phys. Fluids, 26(1):015114, 2014.

[56] J.M. Rallison. The deformation of small viscous drops and bubbles in shear flows. Ann. Rev. Fluid Mech., 16(1):45-66, 1984.

[57] H.A. Stone. Dynamics of drop deformation and breakup in viscous fluids. Annu. Rev. Fluid Mech., 26(1):65-102, 1994.

[58] G.I. Taylor. The viscosity of a fluid containing small drops of another fluid. Proc. Royal Soc. Lond., pages 41-48, 1932.

[59] G.I. Taylor. The formation of emulsions in definable fields of flow. Proc. Royal Soc. Lond., pages 501-523, 1934.

[60] C.E. Chaffey and H. Brenner. A second-order theory for shear deformation of drops. J. Coll. Inter. Sci., 24(2):258-269, 1967.

[61] R. G. Cox. The deformation of a drop in a general time-dependent fluid flow. J. Fluid Mech., 37(03):601-623, 1969.

[62] N.A. Frankel and A. Acrivos. The constitutive equation for a dilute emulsion. J. Fluid Mech., 44(01):65-78, 1970.

[63] A. N. Kolmogorov. On the disintegration of drops in a turbulent flow. In Dokl. Akad. Nauk SSSR, volume 66, page 30, 1949.

[64] J.O. Hinze. Fundamentals of the hydrodynamic mechanism of splitting in dispersion processes. AIChE Journal, 1(3):289-295, 1955.

[65] P. Perlekar, L. Biferale, M. Sbragaglia, S. Srivastava, and F. Toschi. Droplet size distribution in homogeneous isotropic turbulence. Phys. Fluids., 24(6):065101, 2012.

[66] V. Cristini, J. Blawzdziewicz, M. Loewenberg, and L.R. Collins. Breakup in stochastic Stokes flows: sub-Kolmogorov drops in isotropic turbulence. J. of Fluid Mech., 492:231-250, 2003.

[67] L. Biferale, C. Meneveau, and R. Verzicco. Deformation statistics of sub-Kolmogorov-scale ellipsoidal neutrally buoyant drops in isotropic turbulence. J. Fluid Mech., 754:184-207, 2014.

[68] P.L. Maffettone and M Minale. Equation of change for ellipsoidal drops in viscous flow. J. Non-Newtonian Fluid Mech., 78(2):227-241, 1998.

[69] M.U. Babler, L. Biferale, L. Brandt, U. Feudel, K. Guseva, A.S. Lanotte, C. Marchioli, F. Picano, G. Sardina, and A. Soldati. Numerical simulations of aggregate breakup in bounded and unbounded turbulent flows. J. Fluid Mech., 766:104-128, 2015. 
[70] C. Marchioli and A. Soldati. Turbulent breakage of ductile aggregates. Phys. Rev. E, 91(5):053003, 2015.

[71] M.D. de Tullio, J. Nam, G. Pascazio, E. Balaras, and R. Verzicco. Computational prediction of mechanical hemolysis in aortic valved prostheses. Euro. J. Mechanics-B/Fluids, 35:47-53, 2012.

[72] B. Eckhardt, S. Grossmann, and D. Lohse. Scaling of global momentum transport in Taylor-Couette and pipe flow. The Eur. Phys. J. B-Cond. Matter and Comp. Sys., 18(3):541-544, 2000.

[73] B. Eckhardt, S. Grossmann, and D. Lohse. Fluxes and energy dissipation in thermal convection and shear flows. Europhysics Lett., 78(2):24001, 2007.

[74] V. Spandan, R. Ostilla-Mónico, R. Verzicco, and D. Lohse. Drag reduction in numerical two-phase Taylor-Couette turbulence using an Euler-Lagrange approach. J. Fluid Mech., 798:411-435, 72016.

[75] R. Ostilla-Mónico, R. Verzicco, and D. Lohse. Effects of the computational domain size on direct numerical simulations of Taylor-Couette turbulence with stationary outer cylinder. Phys. of Flu., 27(2), 2015.

[76] S. Elghobashi. On predicting particle-laden turbulent flows. App. Sci. Res., 52(4):309-329, 1994.

[77] M. Minale. A phenomenological model for wall effects on the deformation of an ellipsoidal drop in viscous flow. Rheologica Acta, 47(5-6):667-675, 2008.

[78] M. Minale. Models for the deformation of a single ellipsoidal drop: a review. Rheologica acta, 49(8):789-806, 2010.

[79] A. Vananroye, P. Van Puyvelde, and P. Moldenaers. Deformation and orientation of single droplets during shear flow: combined effects of confinement and compatibilization. Rheologica acta, 50(3):231-242, 2011.

[80] S. Guido and M. Villone. Three-dimensional shape of a drop under simple shear flow. J. of Rheology, 42(2):395-415, 1998.

[81] S. Guido, M. Minale, and P.L. Maffettone. Drop shape dynamics under shear-flow reversal. J. Rheology, 44(6):1385-1399, 2000.

[82] T.S. Lund and M.M. Rogers. An improved measure of strain state probability in turbulent flows. Phys. Fluids, 6(5):1838-1847, 1994.

[83] P.H. Mortensen, H.I. Andersson, J.J.J. Gillissen, and B.J. Boersma. On the orientation of ellipsoidal particles in a turbulent shear flow. Int. J. Multiphase Flow, 34(7):678-683, 2008.

[84] C. Marchioli, M. Fantoni, and A. Soldati. Orientation, distribution, and deposition of elongated, inertial fibers in turbulent channel flow. Phys. Fluids, 22(3):033301, 2010. 
[85] D.O. Njobuenwu and M Fairweather. Dynamics of single, non-spherical ellipsoidal particles in a turbulent channel flow. Chem. Eng. Sci., 123:265-282, 2015.

[86] R. Ni, S. Kramel, N. T. Ouellette, and G. A. Voth. Measurements of the coupling between the tumbling of rods and the velocity gradient tensor in turbulence. $J$. Flu. Mech., 766:202-225, 2015.

[87] S Parsa, E Calzavarini, F Toschi, and G A Voth. Rotation rate of rods in turbulent fluid flow. Phys. Rev. Lett., 109(13):134501, 2012.

[88] M. Byron, J. Einarsson, K. Gustavsson, G. Voth, B. Mehlig, and E. Variano. Shape-dependence of particle rotation in isotropic turbulence. Phys. Fluids, 27(3):035101, 2015.

[89] M. Shin and D. L. Koch. Rotational and translational dispersion of fibres in isotropic turbulent flows. Journal of Fluid Mechanics, 540:143-173, 2005.

[90] L. Chevillard and C. Meneveau. Orientation dynamics of small, triaxial-ellipsoidal particles in isotropic turbulence. Jou. of Flu. Mech., 737:571-596, 2013.

[91] S. S. Girimaji and S. B. Pope. Material-element deformation in isotropic turbulence. J. Fluid Mech., 220:427-458, 1990.

[92] P. L. Johnson and C. Meneveau. Large-deviation joint statistics of the finite-time lyapunov spectrum in isotropic turbulence. Phys. Fluids, 27(8):085110, 2015.

[93] R. Clift, J. R. Grace, and M. E. Weber. Bubbles, drops, and particles. Courier Corporation, 2005.

[94] P. Ern, F. Risso, D. Fabre, and J. Magnaudet. Wake-induced oscillatory paths of bodies freely rising or falling in fluids. Ann. Rev. Fluid Mech., 44:97-121, 2012 .

[95] G. Mougin and J. Magnaudet. Path instability of a rising bubble. Phys. Rev. Lett., 88(1):014502, 2001.

[96] R. Zenit and J. Magnaudet. Path instability of rising spheroidal air bubbles: a shape-controlled process. Phys. Fluids, 20(6):061702, 2008.

[97] K. Ellingsen and F. Risso. On the rise of an ellipsoidal bubble in water: oscillatory paths and liquid-induced velocity. J. Fluid Mech., 440:235-268, 2001.

[98] S. Piedra, J. Lu, E. Ramos, and G. Tryggvason. Numerical study of the flow and heat transfer of bubbly flows in inclined channels. Int. J. Heat and Fluid Flow, 56:43-50, 2015.

[99] S. Dabiri and G. Tryggvason. Heat transfer in turbulent bubbly flow in vertical channels. Chem. Eng. Sci., 122:106-113, 2015. 
[100] W-D. Deckwer and R.W. Field. Bubble column reactors, volume 200. Wiley New York, 1992.

[101] R. Latorre, A. Miller, and R. Philips. Micro-bubble resistance reduction on a model ses catamaran. Ocean Engineering, 30(17):2297-2309, 2003.

[102] M. R. Maxey and J. J. Riley. Equation of motion for a small rigid sphere in a nonuniform flow. Phys. Fluids, 26(4):883-889, 1983.

[103] F Toschi and E Bodenschatz. Lagrangian properties of particles in turbulence. Annu. Rev. Fluid Mech., 41:375-404, 2009.

[104] H. Zhang, G. Ahmadi, F. Fan, and J.B. McLaughlin. Ellipsoidal particles transport and deposition in turbulent channel flows. Int. J. Multiphase Flow, 27(6):971-1009, 2001.

[105] C. Yin, L. Rosendahl, S. Knudsen Kær, and H. Sørensen. Modelling the motion of cylindrical particles in a nonuniform flow. Chem. Eng. Sci., 58(15):3489-3498, 2003.

[106] Y. Feng and C. Kleinstreuer. Analysis of non-spherical particle transport in complex internal shear flows. Phys. Fluids, 25(9):091904, 2013.

[107] G.A. Voth and A. Soldati. Anisotropic particles in turbulence. Annu. Rev. Fluid Mech., 49:249-276, 2017.

[108] V. Spandan, R. Verzicco, and D. Lohse. Deformation and orientation statistics of neutrally buoyant sub-kolmogorov ellipsoidal droplets in turbulent taylor-couette flow. J. Fluid Mech., 809(2):480-501, 2016.

[109] G.B. Jeffery. The motion of ellipsoidal particles immersed in a viscous fluid. In Proc. Roy. Soc. Lond. A: Mathematical, Physical and Engineering Sciences, volume 102, pages 161-179. The Royal Society, 1922.

[110] H. Brenner. The Stokes resistance of an arbitrary particle. Chem. Eng. Sci., 18(1):1-25, 1963.

[111] G.H. Ganser. A rational approach to drag prediction of spherical and nonspherical particles. Powder Technology, 77(2):143-152, 1993.

[112] B. Ford and E. Loth. Forces on ellipsoidal bubbles in a turbulent shear layer. Phys. Fluids, 10(1):178-188, 1998.

[113] S. Blaser. Forces on the surface of small ellipsoidal particles immersed in a linear flow field. Chem, Eng. Sci., 57(3):515-526, 2002.

[114] E. Loth. Drag of non-spherical solid particles of regular and irregular shape. Powder Technology, 182(3):342-353, 2008.

[115] Y Feng. Non-spherical particle dynamics analysis with applications to inhaled aerosol transport and deposition in human upper airway models. North Carolina State University, 2013. 
[116] F-G. Fan and G. Ahmadi. Wall deposition of small ellipsoids from turbulent air flows - a brownian dynamics simulation. J. Aerosol Sci., 31(10):1205-1229, 2000.

[117] R. Ouchene, M. Khalij, A. Taniere, and B. Arcen. Drag, lift and torque coefficients for ellipsoidal particles: From low to moderate particle reynolds numbers. Computers \& Fluids, 113:53-64, 2015.

[118] S.F. Hoerner. Fluid-dynamic drag: practical information on aerodynamic drag and hydrodynamic resistance. Hoerner Fluid Dynamics Midland Park, NJ, 1965.

[119] R.Y-S. Lai and L.F. Mockros. The stokes-flow drag on prolate and oblate spheroids during axial translatory accelerations. J. Fluid Mech., 52(01):1-15, 1972.

[120] S. Balachandar and J.K. Eaton. Turbulent dispersed multiphase flow. Ann. Rev. Fluid Mech., 42:111-133, 2010.

[121] J.B. Freund. Numerical simulation of flowing blood cells. Annual Rev. Fluid Mech., 46:67-95, 2014.

[122] F. Sotiropoulos, T.B. Le, and A. Gilmanov. Fluid mechanics of heart valves and their replacements. Ann. Rev. Fluid Mech., 48:259-283, 2016.

[123] R. Mittal, J. H. Seo, V. Vedula, Y. J. Choi, H. Liu, H. H. Huang, S. Jain, L. Younes, T. Abraham, and R. T. George. Computational modeling of cardiac hemodynamics: Current status and future outlook. J. of Comp. Phys., 305:1065-1082, 2016.

[124] E. H. Dowell, R. Clark, and D. Cox. A modern course in aeroelasticity, volume 3. Springer, 2004.

[125] C. S. Peskin. Flow patterns around heart valves: a numerical method. J. Comp. Phys., 10(2):252-271, 1972.

[126] C. S Peskin. The immersed boundary method. Acta numerica, 11:479-517, 2002.

[127] R. Mittal and G. Iaccarino. Immersed boundary methods. Annu. Rev. Fluid Mech., 37:239-261, 2005.

[128] M. Uhlmann and T. Doychev. Sedimentation of a dilute suspension of rigid spheres at intermediate galileo numbers: the effect of clustering upon the particle motion. J. Fluid Mech., 752:310-348, 2014.

[129] F. Picano, W-P. Breugem, and L. Brandt. Turbulent channel flow of dense suspensions of neutrally buoyant spheres. J. Fluid Mechanics, 764:463-487, 2015.

[130] A. Prosperetti. Life and death by boundary conditions. J. Fluid Mech., 768:1-4, 2015. 
[131] W. Fornari, F. Picano, and L. Brandt. Sedimentation of finite-size spheres in quiescent and turbulent environments. J. Fluid Mech., 788:640-669, 2016.

[132] S. Schwarz, T. Kempe, and J. Fröhlich. An immersed boundary method for the simulation of bubbles with varying shape. J. of Comp. Phys., 315:124-149, 2016.

[133] G. Iaccarino and R. Verzicco. Immersed boundary technique for turbulent flow simulations. App. Mech. Rev., 56(3):331-347, 2003.

[134] M.D. de Tullio, P. De Palma, M. Napolitano, and G. Pascazio. Recent advances in the development of an immersed boundary method for industrial applications. In Computational Fluid Dynamics, pages 601-606. Springer, 2011.

[135] M. Uhlmann. An immersed boundary method with direct forcing for the simulation of particulate flows. J. Comp. Phys., 209(2):448-476, 2005.

[136] W-P. Breugem. A second-order accurate immersed boundary method for fully resolved simulations of particle-laden flows. J. Comp. Phys., 231(13):4469-4498, 2012.

[137] M. Vanella and E. Balaras. A moving-least-squares reconstruction for embedded-boundary formulations. J. Comp. Phys., 228(18):6617-6628, 2009.

[138] S Schwarz, T Kempe, and J Fröhlich. A temporal discretization scheme to compute the motion of light particles in viscous flows by an immersed boundary method. J. Comp. Phys., 281:591-613, 2015.

[139] M.D. de Tullio and G. Pascazio. A Moving-Least-Squares immersed boundary method for simulating the fluid-structure interaction of elastic bodies with arbitrary thickness. J. Comp. Phys., 325:201-225, 2016.

[140] C.T. Crowe, T.R. Troutt, and J.N. Chung. Numerical models for two-phase turbulent flows. Ann. Rev. Fluid Mech., 28(1):11-43, 1996.

[141] R. Scardovelli and S. Zaleski. Direct numerical simulation of free-surface and interfacial flow. Ann. Rev. Fluid Mech., 31(1):567-603, 1999.

[142] P. Lancaster and K. Salkauskas. Surfaces generated by moving least squares methods. Mathematics of Computation, 37(155):141-158, 1981.

[143] G.R. Liu and Y.T. Gu. An introduction to meshless methods and their programming. Springer, Berlin, Germany, 2005.

[144] T Belytschko, L Gu, and YY Lu. Fracture and crack growth by element free galerkin methods. Modelling and Sim. in Mat. Sci. and Engg., 2(3A):519, 1994.

[145] T. Belytschko, Y. Krongauz, D. Organ, M. Fleming, and P. Krysl. Meshless methods: an overview and recent developments. Computer Methods in App. Mech. and Engg., 139(1):3-47, 1996.

[146] Y. Krongauz and T. Belytschko. Enforcement of essential boundary conditions 
in meshless approximations using finite elements. Computer Methods in App. Mech. and Engg., 131(1):133-145, 1996.

[147] D. Hegen. Element-free Galerkin methods in combination with finite element approaches. Computer Methods in App. Mech. and Engg., 135(1):143-166, 1996.

[148] S.N. Atluri, J.Y. Cho, and H-G. Kim. Analysis of thin beams, using the meshless local Petrov-Galerkin method, with generalized moving least squares interpolations. Computational Mechanics, 24(5):334-347, 1999.

[149] S. Schaefer, T. McPhail, and J. Warren. Image deformation using moving least squares. In ACM Trans. on Graphics, volume 25, pages 533-540. ACM, 2006.

[150] S. Fleishman, D. Cohen-Or, and C.T. Silva. Robust moving least-squares fitting with sharp features. In ACM Trans. on Graphics, volume 24, pages 544-552. ACM, 2005.

[151] R. Kolluri. Provably good moving least squares. ACM Trans. on Algorithms, $4(2): 18,2008$.

[152] Q-H. Zeng and D-T. LU. Curve and surface fitting based on moving least-squares methods. J. of Engg. Graph., 1(3):84-87, 2004.

[153] L. Kobbelt and M. Botsch. A survey of point-based techniques in computer graphics. Comp. \& Graph., 28(6):801-814, 2004.

[154] J. Yang and E. Balaras. An embedded-boundary formulation for large-eddy simulation of turbulent flows interacting with moving boundaries. J. Comp. Phys., 215(1):12-40, 2006.

[155] A. van Gelder. Approximate simulation of elastic membranes by triangulated spring meshes. Jou. of Graph. Tools, 3(2):21-41, 1998.

[156] M. Chen and F.J. Boyle. Investigation of membrane mechanics using spring networks: Application to red-blood-cell modelling. Material Sci. and Eng. C, 43:506-516, 2014.

[157] D.A. Fedosov, B. Caswell, and G.E. Karniadakis. Systematic coarse-graining of spectrin-level red blood cell models. Computer Methods in App. Mech. and Engg., 199(29):1937-1948, 2010.

[158] M.M. Dupin, I. Halliday, C.M. Care, and L.L. Munn. Lattice Boltzmann modelling of blood cell dynamics. Int. J. Comp. Flu. Dyn., 22(7):481-492, 2008.

[159] T. Krüger. Effect of tube diameter and capillary number on platelet margination and near-wall dynamics. Rheologica Acta, pages 1-16, 2016.

[160] L.D. Landau and E.M. Lifshitz. Fluid mechanics, 1959.

[161] M. Emans and C. Zenger. An efficient method for the prediction of the motion of individual bubbles. Int. J. Comp. Fluid Dynamics, 19(4):347-357, 2005. 
[162] S. Torza, R.G. Cox, and S.G. Mason. Particle motions in sheared suspensions xxvii. transient and steady deformation and burst of liquid drops. J. Coll. Int. Sci., 38(2):395-411, 1972.

[163] E Loth. Quasi-steady shape and drag of deformable bubbles and drops. Int. J. Multi. Flow, 34(6):523-546, 2008.

[164] M. Uhlmann. Simulation of particulate flows on multi-processor machines with distributed memory. Ciemat, 2004.

[165] S. Wang, G. He, and X. Zhang. Parallel computing strategy for a flow solver based on immersed boundary method and discrete stream-function formulation. Computers \& Fluids, 88:210-224, 2013.

[166] E.H. Dowell and K.C. Hall. Modeling of fluid-structure interaction. Annu. Rev. Fluid Mech., 33(1):445-490, 2001.

[167] V. Spandan, V. Meschini, R. Ostilla-Monico, D. Lohse, G. Querzoli, M.D. de Tullio, and R. Verzicco. A parallel interaction potential approach coupled with the immersed boundary method for fully resolved simulations of deformable interfaces and membranes. arXiv preprint arXiv:1612.03399, 2016.

[168] H. Sigurgeirsson, A. Stuart, and W-L. Wan. Algorithms for particle-field simulations with collisions. J. Comp. Phys., 172(2):766-807, 2001.

[169] M.P. Allen and D.J. Tildesley. Computer simulation of liquids. Oxford university press, 1989.

[170] S. Thomas, A. Esmaeeli, and G. Tryggvason. Multiscale computations of thin films in multiphase flows. Int. J. Multiphase Flow, 36(1):71-77, 2010. 


\section{Summary}

Multiphase turbulent flows are characterised by a wide range of length and time scales in addition to the complex non-linear interactions between the different phases in the flow. Advances in theoretical and experimental techniques have helped in clarifying many non-intuitive and puzzling phenomena observed in multiphase flows. However, they fall short in certain aspects which has been fulfilled with the advent of numerical simulations. While numerical simulations are extremely versatile and give in depth understanding of the problem at hand, given its highly non-trivial nature, it is not possible to devise a single universal numerical tool which can be used to study all kinds of multiphase flows. In this thesis, we study the dynamics of bubbles and drops dispersed in a turbulent Taylor-Couette (TC) flow. The complexity of scale separation in such a flow led to the development and use of a variety of efficient and versatile numerical tools which were used to understand the flow dynamics in a two-phase TC system.

In $\S 2$, we start by understanding the influence of sub-Kolmogorov spherical bubbles on the overall drag in TC flow. A point-particle Euler-Lagrange approach is used to model the motion of the bubbles while the dynamics of the carrier fluid is computed using direct numerical simulations. Two-way coupling between the carrier fluid and the dispersed bubbles is crucial to understand the influence of the bubbles on the coherent flow structures that arise in TC flow. In particular, we found that low Froude number bubbles (i.e buoyancy is strong in comparison to the centripetal force) are more active in reducing the drag in the system in comparison to high Froude number bubbles. The motion of low Froude number bubbles through the plume ejection regions on the inner cylinder and the coherent Taylor rolls in the bulk results in their weakening which ultimately leads to a lower angular velocity transport in the system or in other words lower torque on the rotating cylinder. This effect is clearly seen in the time-averaged contours of the azimuthal velocity and also trajectories of individual bubbles in the flow. While the point-particle Euler-Lagrange approach can be used to study $\mathrm{O}\left(10^{5}\right)$ sub-Kolmogorov dispersed bubbles/drops/particles in a turbulent flow, it constrains the shape of the dispersed phase to be fixed (typically either spherical or ellipsoidal) throughout the simulation. This hurdle is overcome in $\S 3$ and $\S 4$. 
In $\S 3$, we couple Euler-Lagrange tracking with a phenomenological sub-grid deformation model to study the deformation dynamics of $10^{5}$ sub-Kolmogorov neutrally buoyant drops in TC flow. The dispersed drops are considered to be ellipsoidal and are advected as passive tracers in the flow. The surface of each drop is represented through a unique second-order positive-definite symmetric shape tensor. The time evolution of the shape tensor depends on the local strain-rate, vorticity in the flow, Capillary number and also the viscosity ratio of the drops to the carrier fluid. Increasing the capillary number leads to an increase in the deformation of the dispersed ellipsoidal drops. When the viscosity ratio of the drops to the carrier fluid is equal to one, the drops tend to be prolate or (cigar-like) due to preferential stretching in the stream-wise direction. The major-axis of the elongated drops tend to align with the stream-wise direction in the inner boundary layer, while the orientational preference is close to isotropic in the bulk. On increasing the viscosity ratio, the drops tend to be more oblate (disk-like) in the boundary layers due to increased influence of rotation. In order to extend the study in $\S 3$ from drops to bubbles, we couple the sub-grid deformation model to a momentum equation for ellipsoidal particles along with two-way coupling between the dispersed bubbles and the carrier fluid. This is studied in $\S 4$, where we focus on understanding the influence of deformability of sub-Kolmogorov bubbles on the torque on the rotating cylinder in TC flow. On increasing the deformability of the dispersed bubbles (which is achieved by increasing the Capillary number of the bubbles in the simulation) we find a net increase in the drag reduction obtained in a two-phase TC system. Deformed bubbles tend to accumulate more near the inner cylinder in comparison to spherical undeformed bubbles. A higher volume fraction of bubbles close to the inner cylinder implies that they are more effective in weakening the plume ejections and consequently the strength of the coherent Taylor rolls. In $\S 2, \S 3$ and $\S 4$, we focused on sub-Kolmogorov drops or bubbles which are either spherical or have limited degrees of freedom during deformation. However, in experiments it is not straight-forward to independently control the diameter of the dispersed bubbles and drops in TC flow and situations may arise where the drops or bubbles are much larger the Kolmogorov length scale. Point particle Euler-Lagrange type approaches are not valid for bubbles/drops in this regime and this hurdle is overcome with the development of a parallelised moving least squares immersed boundary method with an interaction potential deformation in $\S 5$ and $\S 6$.

The immersed boundary method has taken over the fluid dynamics community in the last few decades and in particular the field of computational multiphase flows. While several advances have been made in simulating $\mathrm{O}\left(10^{3}\right)$ finite-size 
spherical particles in turbulent flows, a major challenge has been the inclusion of deformability of the dispersed particles. In $\S 5$, we describe the merging of a parallelised finite-difference Navier-Stokes solver with an immersed boundary method based on a moving least squares approximation and an interaction potential approach for deformation. Such a solver is capable of handling $\mathrm{O}\left(10^{4}\right)$ deformable bodies with several degrees of freedom in highly turbulent flows. A unique feature of this approach in comparison to conventional techniques used to simulate finite size immersed drops or bubbles is its computational efficiency which allows us to scale up both in terms of the number of drops or bubbles in the flow and also the turbulence levels in the surrounding fluid. Parallelisation of the solver is inevitable while handling large scale flow systems and to this effect we describe an efficient and easy-to-implement parallelisation scheme which exhibits strong scaling up to $10^{4}$ cores with MPI and OpenMP directives. In $\S 6$, we further enhance the algorithms described in $\S 5$, with a particular focus on scaling up the capability of the solver in terms of the number of dispersed deformable bodies that can be simultaneously simulated in a turbulent flow. This is achieved by implementing a fast-moving least squares approximation technique which eliminates the need for the inversion of a coefficient matrix for the triangular elements present on the dispersed bodies. An adaptive Lagrangian mesh refinement procedure tackles the constraint of maintaining a uniform Eulerian grid resolution throughout the domain. Additionally, a coarse-grained collision detection algorithm is presented where the computational overhead on the overall flow solver is less than $2 \%$ for any number of dispersed bodies present in the flow.

We use the algorithms presented in $\S 5$ and $\S 6$ to study the dynamics of finite-size bubbles and drops in TC flow in $\S 7$. We vary the Weber number of the dispersed bubbles and drops and study its influence on the net torque on the rotating solver. The net drag reduction attained is computed in the form of mean dissipation in the fluid and we find that increasing deformability of finite-size bubbles leads to increase in the drag reduction. However, unlike $\S 4$, the bubble distribution within the cylinders is not affected by the deformability. In the case of finite-size bubbles, drag reduction is a result of an effective laminarisation of the flow in the bulk and this is shown through time averaged radial profiles of the angular velocity transport. Upon the injection of finite-size neutrally buoyant drops we find drag enhancement in the system due to the dissipation in the fluid enclosed within the interface. We find similar trends in drag reduction by bubbles in the case of turbulent boundary layers. 


\section{Samenvatting}

Turbulente meerfasestromingen worden gekenmerkt door een breed scala aan lengte- en tijdschalen en door complexe niet-lineaire interacties van de verschillende fasen in de stroming. Door de vooruitgang op theoretisch en experimenteel vlak, konden niet-intuïtieve en raadselachtige fenomenen die zijn waargenomen in meerfasenstromingen worden verklaard en verduidelijkt. Echter waren er op sommige aspecten tekortkomingen welk door de komst van numerieke simulaties konden worden aangevuld. Numerieke simulaties zijn erg veelzijdig en helpen bij het creëeren van begrip en nieuwe inzichten. Gezien zijn complexe natuur, is het niet mogelijk om een universeel numeriek hulpmiddel te gebruiken om de vele verschillende meerfasenstromingen te bestuderen. In dit proefschrift bestuderen wij de dynamica van bellen en druppels in een turbulente Taylor-Couette (TC) stroming. De complexiteit dat de scheiding van verschillende schalen in een dergelijke vloeistofstroom met zich mee bracht leidde tot de ontwikkeling van een aantal efficiënte veelzijdige numerieke hulpmiddelen die hebben bijgedragen aan het begrip van de stromingsdynamica in een tweefases TC systeem.

In hoofdstuk 2 kijken we naar de invloed van ronde sub-Kolmogorov bellen op de gemiddelde wrijving in de TC stroming. Euler-Lagrange puntdeeltjes zijn gebruikt om de bewegingen van de bellen te modelleren terwijl de dynamica van de draagvloeistof is berekend met directe numerieke simulaties. Tweeweg koppeling tussen de draagvloeistof en de bellen is cruciaal om de invloed van de bellen op de coherente structuren in de TC stroming te begrijpen. Wij ontdekten dat bellen met een laag Froude getal (opwaartse kracht groter dan de centripetale kracht) beter in staat zijn om de wrijving te verminderen dan bellen met een hoog Froude getal. Bellen met een laag Froude getal die bewegen door gebieden waar pluimen worden geïnjecteerd zorgen voor onderdrukking van dit effect, dat resulteert in de onderdrukking van Taylorrollen en uiteindelijk tot een lager hoeksnelheidstransport, met andere woorden een lager benodigd koppel om de binnencilinder te roteren. Dit effect is duidelijk zichtbaar in the contouren van de tijdsgemiddelde azimutale snelheid en in het afgelegde pad van de individuele bellen in de stroming. De puntdeeltjes Euler-Langrange methode kan worden gebruikt tot Reynolds nummers van orde $10^{5}$ en sub-Kolmogorov bellen, druppels, en deeltjes in turbulente stromingen, echter de 
vorm van de dispergeerde fase is beperkt tot enkel bolvormig of ellipsoïde. Deze horde wordt genomen in hoofdstuk 3 en 4 .

In hoofdstuk 3 koppelen wij de Euler-Lagrange methode aan een subrooster vervormbaarheidsmodel om de vervormingsdynamica te bestuderen van neutrale sub-Kolmogorov druppels in een TC stroming en een Reynolds nummer tot $10^{5}$. We nemen aan dat de druppels een elliptische vorm hebben en dat ze als passieve volgers worden meegenomen met de stroming. Het oppervlak van elke druppel wordt gerepresenteerd door een unieke tweede-orde positief eindige symmetrische vormtensor. De tijdsevolutie van de vormtensor hangt af van lokale schuifweerstand, vorticiteit, het capillaire getal, en de viscositeitsratio van de druppels en de draagvloeistof. Een verhoging van het capillaire getal leidt tot het vergroten van de vervormbaarheid van de verspreide elliptische druppels. Als de viscositeitsratio van de druppels ten opzichte van de draagvloeistof gelijk is aan één, hebben de druppels de neiging zich uit te rekken (sigaarachtige vorm) door de voorkeursrek van de stromingsrichting. De hoofdas van de uitgerekte druppels richt zich in de richting van de stroming in de binnenste grenslaag terwijl de oriëntatie van de druppels in de bulkstroming zo goed als isotroop verdeeld is. Bij een toenemende viscositeitsratio zullen de druppels aan de polen meer worden afgeplat (schijfvormig) in de grenslagen door de vergrote invloed van de rotatie. Om voor deze studie over te gaan van druppels naar bellen koppelen wij het subrooster vervormbaarheidsmodel aan een impulsvergelijking voor elliptische deeltjes samen met een tweeweg koppeling tussen de verspreide bellen en de draagvloeistof. Dit werd gedaan in hoofdstuk 4 waarbij de focus lag op het begrijpen van de invloed van vervormbaarheid van sub-Kolmogorov bellen op het koppel van de roterende cilinder in een TC stroming. Door een toename in vervormbaarheid van de verspreide bellen (dat werd bewerkstelligd door het vergroten van het capillaire getal van de bellen in de simulatie) zien wij een netto afname van de weerstand van een tweefasen TC systeem. Vervormde bellen neigen zich te verzamelen dichtbij de binnencilinder, in tegenstelling tot ronde niet-vervormbare bellen. Een hogere volumefractie van bellen dichtbij de binnencilinder impliceert dat deze effectiever zijn in het onderdrukken van pluimejecties en dus daarmee ook de kracht van de coherente Taylorrollen. In hoofdstuk 2, 3, en 4 was de focus op sub-Kolmogorov druppels en bellen die dan wel rond, of een beperkte vrijheid van vervorming hebben. In TC experimenten is het niet eenvoudig om onafhankelijk de diameter van verspreide bellen of druppels in te stellen en kunnen er situaties optreden waarbij deze diameters duidelijk groter zijn dan de Kolmogorov lengteschaal. De puntdeeltjes Euler-Langrange-type methodes zijn niet geldig voor bellen/druppels in dit regime. In hoofdstuk 5 en 6 wordt beschreven hoe dit 
probleem kan worden overwonnen door de ontwikkeling van een geparallelliseerde voortschrijdende kleinste-kwadraten ondergedompelde grensmethode (IBM) met een vervormbaarheid gebaseerd op een interactiepotentiaal.

De IBM methode heeft in de afgelopen decennia in vloeistoffysicagemeenschap erg aan populariteit gewonnen, vooral in het veld van computationele meerfasenstromingen. Terwijl er veel vooruitgang is geboekt met het simuleren van $\mathrm{O}\left(10^{3}\right)$ ronde deeltjes met een eindige grootte in turbulente stromingen, was de grootste uitdaging het toevoegen van vervormbaarheid aan de verspreide deeltjes. In hoofdstuk 5 beschrijven wij een geparallellisseerde eindige-differentie Navier-Stokes oplosalgoritme gecombineerd met een IBM gebaseerd op een voortschrijdende kleinste-kwadratenapproximatie en vervormbaarheid gebaseerd op een interactiepotentiaal. Een dergelijk oplosalgoritme is in staat om $\mathrm{O}\left(10^{4}\right)$ vervormbare lichamen met meerdere vrijheidsgraden in hoog turbulente stromingen te verwerken. Een unieke eigenschap van deze methode vergeleken met andere methodes die eindige grootte bellen/druppels simuleren is de efficiëntie van de berekeningen waardoor we niet alleen het aantal bellen of druppels omhoog kunnen schalen, maar ook de mate van turbulentie van de omringende vloeistof. Voor het verwerken van grootschalige vloeistofsystemen is het onvermijdelijk om het algoritme te parallelliseren. Ons algoritme is makkelijk implementeerbaar en heeft een sterke schaling tot maar liefst $10^{4}$ rekenkernen, waarbij MPI en OpenMP worden gebruikt. In hoofdstuk 6 breiden wij het algoritme beschreven in hoofdstuk 5 nog meer uit, met als doel om het aantal verspreide vervormbare lichamen die simultaan in de turbulente stroming kunnen worden gesimuleerd verder op te schalen. Dit is bereikt door het implementeren van een snellopende kleinste-kwadratenapproximatietechniek waarvoor een inversie van de coëfficiëntmatrix voor alle driehoekselementen van de verspreide lichamen overbodig maakt. Een adaptieve Lagrangiaanse roosterverfijningstechniek verwerpt de beperking van constant onderhouden van een uniform Euleriaans rooster voor het gehele domein. Daar bovenop is een grofgeroosterd botsingsdetectiealgoritme gebruikt waarvoor de computationele overhead op het gehele simulatiesysteem minder is dan $2 \%$ for elk willekeurig aantal verspreide lichamen aanwezig in de vloeistof.

Wij gebruiken het algoritme gepresenteerd in hoofdstuk 5 en 6 voor het bestuderen van de dynamica van eindig-grootte bellen en druppels in TC in hoofdstuk 7. Hierbij variëren wij het Weber getal van de verspreide druppels en bellen en bestuderen de invloed hiervan op het koppel op de cilinder in de simulatie. De netto weerstandsvermindering is berekend in de vorm van gemiddelde dissipatie van de vloeistof en hiermee vonden wij dat een vergrote vervormbaarheid van 
de eindig-grootte bellen resulteert in meer weerstandsvermindering. Echter, in tegenstelling tot hoofdstuk 4 , heeft de vervormbaarheid van de bellen geen invloed op de distributie van de verspreide bellen. In het geval van de eindig-grootte bellen is de weerstandsvermindering het resultaat van een effectieve laminarisatie in het overgrote deel van de stroming. Dit is aangetoond door de tijdgemiddelde radiale profielen van het hoeksnelheidstransport. Door injectie van neutrale eindig-grootte druppels zien wij juist een toename van de weerstand in het systeem wat kan worden toegerekend aan de dissipatie van de ingesloten vloeistof. Wij vinden een vergelijkbare trend voor weerstandsvermindering van bellen in het geval van turbulente grenslagen. 


\section{Scientific Output}

\section{Publications}

- Vamsi Spandan, Rodolfo Ostilla-Monico, Detlef Lohse, Roberto Verzicco Identifying coherent structures and vortex clusters in Taylor-Couette turbulence Journal of Physics: Conference Series, Vol. 708, 012006, 2016. (open-access)

- Vamsi Spandan, Rodolfo Ostilla-Monico, Roberto Verzicco, Detlef Lohse Drag reduction in numerical two-phase Taylor-Couette turbulence using an Euler-Lagrange approach

Journal of Fluid Mechanics, Vol. 798, 411-435, 2016.

(https://arxiv.org/abs/1510.01107)

- Vamsi Spandan, Detlef Lohse, Roberto Verzicco Deformation and orientation statistics of neutrally buoyant sub-Kolmogorov ellipsoidal droplets in turbulent Taylor-Couette flow

Journal of Fluid Mechanics, Vol. 809, 480-501, 2016.

(https://arxiv.org/abs/1606.03835)

- Vamsi Spandan, Valentina Meschini, Rodolfo Ostilla-Monico, Detlef Lohse, Giorgio Querzoli, Marco D. de Tullio, Roberto Verzicco A parallel interaction potential approach coupled with the immersed boundary method for fully resolved simulations of deformable interfaces and membranes under review in Journal of Computational Physics (https://arxiv.org/abs/1612.03399)

- Vamsi Spandan, Roberto Verzicco, Detlef Lohse Deformable ellipsoidal bubbles in Taylor-Couette flow with enhanced Euler-Lagrange tracking under review in Physical Review Fluids

- Vamsi Spandan, Detlef Lohse, Marco D. de Tullio, Roberto Verzicco A fast moving least squares approximation with adaptive Lagrangian mesh refinement for large scale immersed boundary simulations under review in Journal of Computational Physics 
- Rodolfo Ostilla-Monico, Xiaojue Zhu, Vamsi Spandan, Roberto Verzicco, Detlef Lohse Three life stages of wall-bounded decay of Taylor-Couette turbulence under review in Physical Review Fluids (https://arxiv.org/abs/1704.07892)

- Xiaojue Zhu, Everett Phillips, Vamsi Spandan, John Donners, Gregory Ruetsch, Josh Romero, Rodolfo Ostilla-Monico, Yantao Yang, Detlef Lohse, Roberto Verzicco, Massimiliano Fatica, Richard J.A.M. Stevens AFiD-GPU: a versatile Navier-Stokes Solver for Wall-Bounded Turbulent Flows on GPU Clusters under review in Computer Physics Communications (https://arxiv.org/abs/1705.01423)

- Vamsi Spandan, Roberto Verzicco, Detlef Lohse Finite-size bubbles and drops in a turbulent Taylor-Couette flow manuscript under preparation

\section{Contributed Talks}

- 2017

- FOM-Physics, Veldhoven, Netherlands

- 2016

- APS-Division of Fluid Dynamics, Portland, USA.

- European Fluid Mechanics Conference, Sevilla, Spain.

- Int. Conf. Multiphase Flows, Florence, Italy.

- 2015

- APS-Division of Fluid Dynamics, Boston, USA.

- Dispersed Two-Phase Flow Meeting, Toulouse, France.

- European Turbulence Conference, Delft, Netherlands.

- Multiflow Summer School, Madrid, Spain.

\section{Invited Talks and Lectures}

- Multiscale Mechanics Group, Univ. Twente, Netherlands

- Multiphase Flows, Graduate level course, Univ. Twente, Netherlands 


\section{Grants and Awards}

- ACRI Young Investigator Training Grant (2016).

- Second place at SURF Visualisation Challenge, SuperD, Amsterdam (2016).

- Computational time on PizDaint, CSCS (Switzerland) (2016-17).

- European PRACE projects on Fermi and Marconi, CINECA (Italy) (2015-17).

- Computational time on Lisa and Cartesius, SurfSARA (Netherlands) (2014-).

\section{Teaching Experience}

- Lecturer and Teaching Assistant, Turbulence (2014-17), Univ. Twente.

- Teaching Assistant, Dynamic Modelling and Simulation (2013-14), Univ. Twente.

\section{Workshops}

- 2016: Dispersed Two-Phase Flows, JMBC, Delft, Netherlands.

- 2016: Small Scale Modelling and Simulation of Turbulent Multiphase Flows, CISM, Udine, Italy.

- 2015: Multiflow Summer School, Technical University of Madrid, Madrid, Spain.

- 2014: $23^{\text {rd }}$ Summer School on Parallel Computing, CINECA, Bologna, Italy.

- 2014: Lorentz Center Workshop on 'Rainfall and Marine Snow: How small scale physical interactions have large consequences'. 
Scientific Output 


\section{Acknowledgments}

I first met Detlef and Roberto in connection with the FOM-CSER PhD program at the APS-DFD meeting in San Diego, USA in 2012. It was an overwhelming experience not only because I was in a new country for the first time but also because of the scale of the meeting and the expertise around. After a discussion about the project and also meeting with a few students from the group, I was convinced that I wanted to start my $\mathrm{PhD}$ with Detlef and Roberto. Detlef offered me the opportunity for a $\mathrm{PhD}$ position in his group after attending my talk and the rest of the formalities related to the FOM-CSER proposal fell into place soon afterwards.

Dear Detlef, I have learnt a lot from you in these past four years on how to understand a scientific problem and to convey it properly. You have not only given me freedom during this period but also have corrected me whenever I went in the wrong direction and this was crucial in successfully completing this thesis. I greatly appreciate the confidence you have shown in me and this has really helped me in pushing the boundaries of this thesis and also other ongoing projects.

Dear Roberto, whenever I was in doubt or hit a sort of dead-end during my $\mathrm{PhD}$, all I had to do was to speak to you. Our discussions always made me excited about tackling the challenge at hand. I really enjoyed my visits to Rome, where our discussions led to new and exciting ideas. Your support, guidance and thoughtfulness has been important at every stage of this project. You also initiated the collaboration with Marco and Valentina and this has improved the thesis a great deal which I could not have possibly imagined at the start of my $\mathrm{PhD}$.

Dear Marco, a major part of this thesis would not have been possible without your help and guidance. I am always amazed at your prompt replies to my questions regardless of its simplicity or complexity. I also really enjoyed my visit to Bari, where we started our collaboration. Dear Valentina, I always enjoy our scientific and non-scientific chats and I have learnt a lot from our collaboration. I wish you all the best for your future endeavours.

This brings me to my colleagues at PoF. Numerical programming can sometimes be quite stressful and it is always soothing to know that there is always someone to help out. Rodolfo, I have learnt a lot from you and would like to thank you for all the help during my $\mathrm{PhD}$. We have come a long way from the original Code409 
version I started with and your support has been extremely important. Erwin, I would like to thank you for helping me out whenever I got stuck with the code during the initial years of my $\mathrm{PhD}$. Yantao, I have always admired your scientific expertise and incisive thinking. I could approach you with any question and you always gave useful insights. Xiaojue, our scientific and non-scientific discussions are always fun. Richard, your persistence in maintaining the codes and the computational times is admirable and beneficial to the whole numerical team. Alexander, I admire your courage in taking up the challenge of large scale simulations and Pieter, for your enthusiasm. I wish you both all the best for your PhD's.

I would like to thank the SurfSARA, PRACE consortium and other HPC organisations for providing us with computing time and technical support whenever required. Bas and Joanita, thanks for all the assistance you provided during my $\mathrm{PhD}$.

During the course of my stay in Enschede, I have met many people who have been great friends and to whom I have been very thankful: (In alphabetical order) Adeline, Anais, Anupam, Dennis v G., Diana, Elise, Fede, Guillaume, Ivan, Maazi, Maxime, Nakul, Paul, Peter, Poorvi, Ricardo, Rodrigo, Roeland, Ruben, Sander H., Sander W., Shantanu, Utkarsh, Varghese, Vivek. Rianne, C-W, Loreto, it was fun sharing the office with you. Dennis B., thanks for translating the summary of this thesis and also co-organising the python workshops. Leo, Loreto, Kike, thanks for all the fun plans and experiences we have shared over the last four years. Biljana, you have been a great support to me in the last three years to which I am very grateful.

Finally, I would like to thank my parents who have worked hard to provide me with everything I need. Also thanks to my sister who, along with my parents, has supported me at every point of my life. 